\author{
Dyuti Sarker \\ Bachelor of Science in Materials and Metallurgical Engineering \\ Bangladesh University of Engineering and Technology, 2006 \\ Master of Science in Materials Engineering \\ International Islamic University Malaysia, 2010
}

\author{
A dissertation \\ presented to Ryerson University \\ in partial fulfillment of the \\ requirements for the degree of \\ Doctor of Philosophy \\ in the Program of \\ Mechanical and Industrial Engineering
}

Toronto, Ontario, Canada, 2014

CDyuti Sarker, 2014 


\section{AUTHOR'S DECLARATION}

I hereby declare that I am the sole author of this dissertation. This is a true copy of the dissertation, including any required final revisions, as accepted by my examiners.

I authorize Ryerson University to lend this dissertation to other institutions or individuals for the purpose of scholarly research.

I further authorize Ryerson University to reproduce this dissertation by photocopying or by other means, in total or in part, at the request of other institutions or individuals for the purpose of scholarly research.

I understand that my dissertation may be made electronically available to the public. 


\title{
ABSTRACT \\ Twinning and Texture Development in an Extruded AM30 Magnesium Alloy during Compressive Deformation
}

Doctor of Philosophy, 2014

Dyuti Sarker

\author{
Mechanical and Industrial Engineering \\ Ryerson University
}

This study was aimed at evaluating the microstructure, mechanical behavior and texture response of extruded AM30 magnesium alloy in uniaxial compression with special attention to the effect of compressive strain amount, sample orientation, loading direction, compressive prestrain, and annealing. Compressive deformation along the extrusion direction (ED) resulted in sigmoidal true stress-true strain behavior together with three distinct stages of strain hardening, due to the presence of two sets of basal textures $\{0001\}<2 \overline{1} \overline{1} 0>$ and $\{0001\}<10 \overline{1} 0>$, with $c$ axes aligned nearly parallel to the normal direction (ND) of the extruded plate which facilitated the occurrence of $\{10 \overline{1} 2\}$ extension twinning. The effect of in-plane loading direction, i.e., $0^{\circ}$, $15^{\circ}, 30^{\circ}, 45^{\circ}$ from the ED on the compressive flow behavior was investigated, which revealed nearly constant yield and ultimate compressive strengths but with a remarkable increase of fracture strain compared to the ED sample. The effect of pre-strain along the ED on the formation of twinning and texture was investigated during re-compression along the ED, 
transverse direction (TD) and ND. In the two-step ED-ED compression, the disappearance of twin boundaries or the coalescence of twins via twin growth was observed. After pre-strain along the ED, re-compression along the TD showed two seemingly opposite phenomena, i.e., the formation of new twins and de-twinning to be co-existent due to the presence of multiple sets of textures after the first-step pre-straining. The de-twinning activity decreased and the texture weakening was achieved with increasing pre-strain in the ED while doing recompression along the ND at a constant strain amount. Texture measurements revealed that the $c$-axes of hcp unit cells were always rotated towards the compression direction, regardless of compression in the ED, TD or ND. The annealing temperature and time also had a pronounced effect on microstructure and texture evolution. With increasing annealing time, the twins in the pre-compressed samples were observed to disappear gradually, as demonstrated by a decreased volume fraction of twins and weakened texture which became more randomly distributed. As a result, during re-compression along the ED, fewer twin formation and less intense texture were observed, resulting in a significant increase of ductility. 


\section{ACKNOWLEDGEMENTS}

Foremost, I wish to express my deepest gratitude to my research supervisors, Dr. Daolun Chen and Dr. Jacob Friedman, for their guidance and support since my very first day at Ryerson, and for providing me with every opportunity and believing in me in all aspects of my education. I am indebted to them for their constant support, guidance and inspiration throughout this work. The completion of this project would not have been possible without their continued guidance and support; of which I am extremely grateful and realize what extraordinary advisors they have been. Their leadership, support, prompt response, dedication to hard work, is a steady source of encouragement to me. While being the beacon light for my path to graduation, they have not only taught me aspects of materials science, but have supported me, oriented me and often engaged me discussions that have led to further insights. I will be eternally grateful to my supervisors for nurturing me carefully with useful research discussions, teaching me how to identify and select compelling research problems, set a high research standard and put unyielding efforts to achieve the final goal. Undoubtedly, they have been instrumental in shaping my knowledge and my abilities as a researcher.

I am grateful to my committee members Dr. S.D. Bhole, Dr. Hua Lu and Dr. Marcello Papini for their precious time in evaluating and subsequently raising several valuable points during the course of this work. Their constructive criticism has greatly helped me to improve the quality of the presentation of this thesis. 
I would like to thank the Natural Sciences and Engineering Research Council of Canada (NSERC), Premier's Research Excellence Award (PREA), NSERC-DAS Award, AUTO21 Network of Centers of Excellence, Automotive Partnership Canada, and Ryerson Research Chair Program for providing financial support. I would also thank Dr. A.A. Luo from Ohio State University (formerly with General Motors Research and Development Center) for the supply of extruded AM30 magnesium alloy, and Dr. R. Tandon and Dr. B. Davies (Magnesium Elektron) for supplying magnesium powders for the defocusing calibration.

Many thanks go out to the staff at Ryerson University's Department of Mechanical and Industrial Engineering. My special thanks to Mr. Joseph Amankrah, for help in specimen preparation, to Mr. Chao Ma, for his unforgettable help to use the equipment, Mr. Qiang Li, always his help in critical analysis and group discussion. Many people helped me on many aspects that are indispensable; in particular, the great assistance by Mr. Alan Machin, Mr. A. Heim, and R. Churaman has been vital in completing this thesis. I am humbled by their considerable help that they rendered to solve many problems with my equipments. Their experience, help and courteous behavior were often a source of inspiration and hope at critical times. I would like to express my gratitude to Program administrator Dahlia Younan for her efficient delivery in graduate programs and all of my friends for their kindness throughout this work and also for providing the friendly environment in our group during the past years of study. 


\section{DEDICATION}

This work is dedicated to my husband, Sugrib Kumar Shaha, who has been giving me lasting loves, continuous supports and constant understandings. And also, to my parents, Prem Chand Sarker and Ratna Sarker, my father- and mother-in-law, Subodh K Shaha and Archona Shaha, and the rest of my family, for their believing in me, inspiring me, and encouraging me. 


\section{TABLE OF CONTENTS}

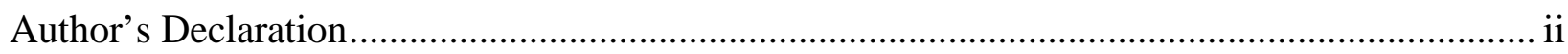

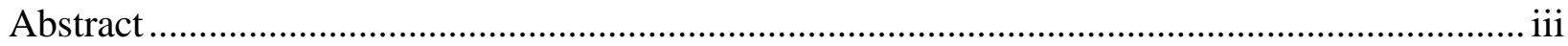

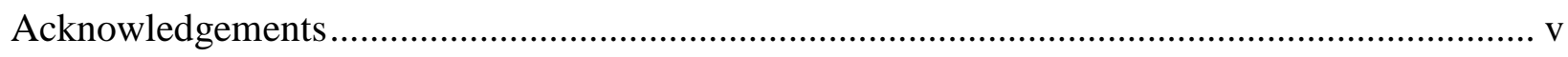

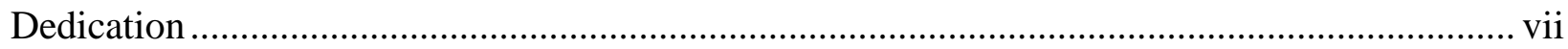

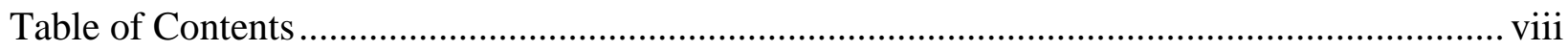

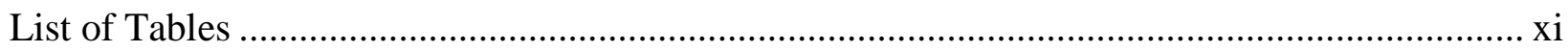

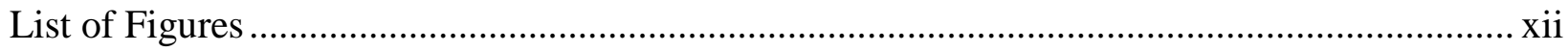

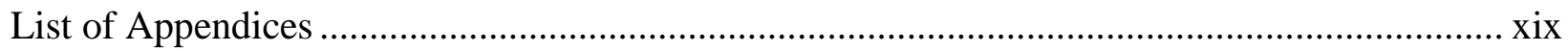

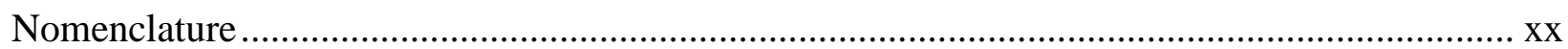

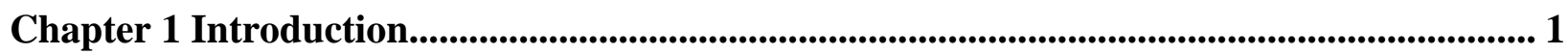

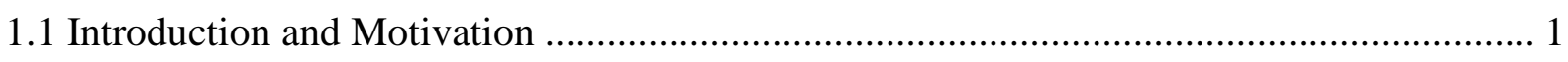

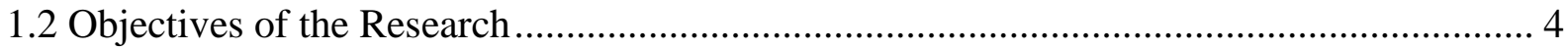

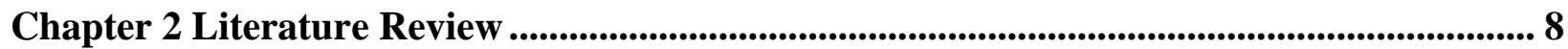

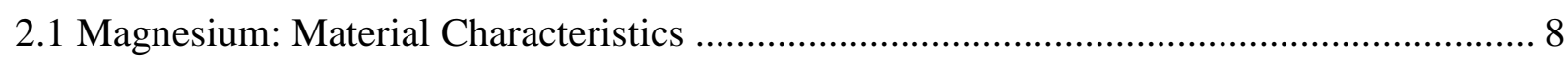

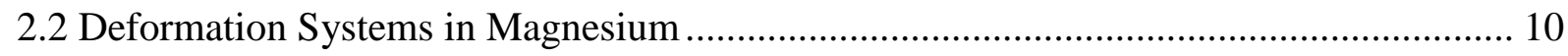

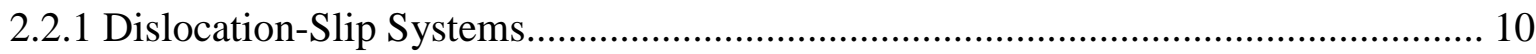

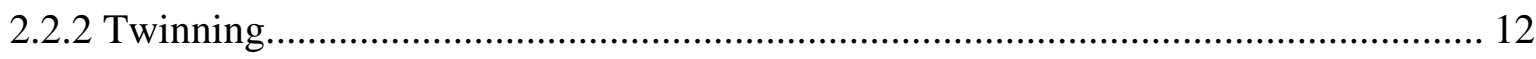

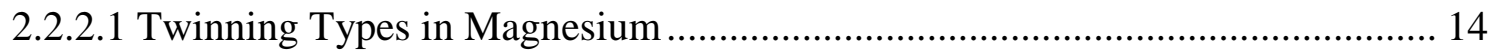

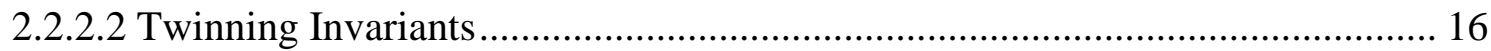

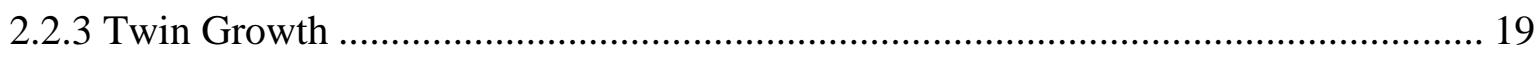

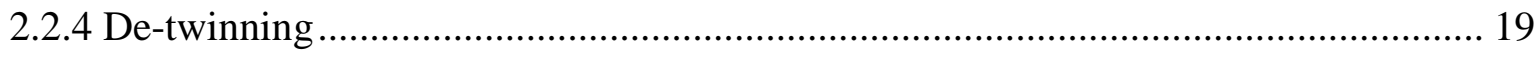

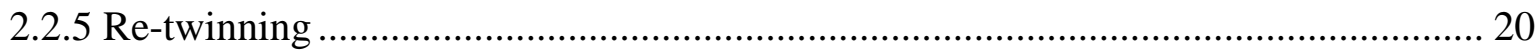

2.3 Factors Influencing Deformation Mechanisms ........................................................... 21

2.3.1 Critical Resolved Shear Stress....................................................................... 21

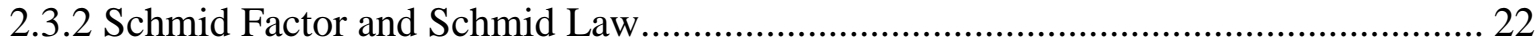

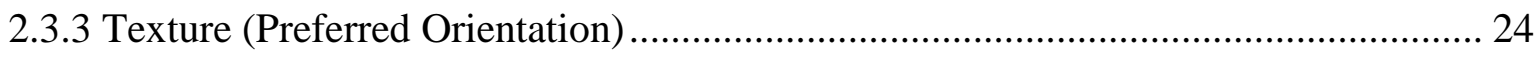

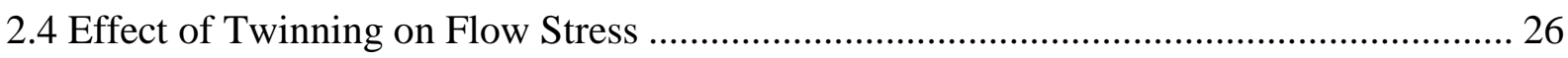

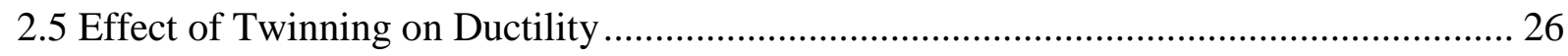




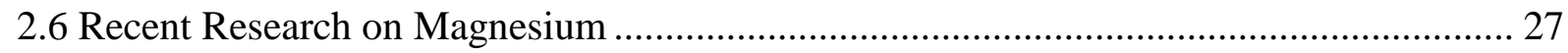

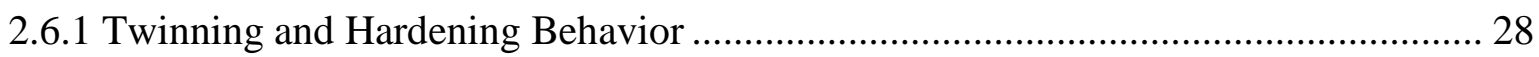

2.6.2 Texture Effect on Deformation Mechanisms ...................................................... 29

2.6.3 Effect of Pre-compression and Loading Direction on Twinning and De-twinning ... 30

2.6.4 Annealing and Its Influence on Twinning and Texture.............................................. 33

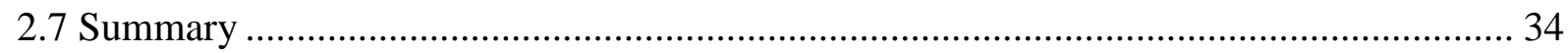

Chapter 3 Materials and Experimental Details ..................................................................................... 37

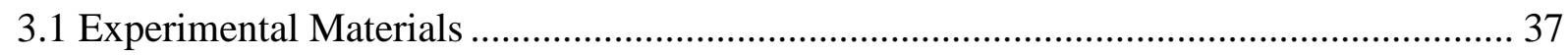

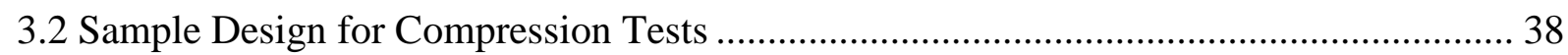

3.3 Uniaxial Compression Tests ...................................................................................... 39

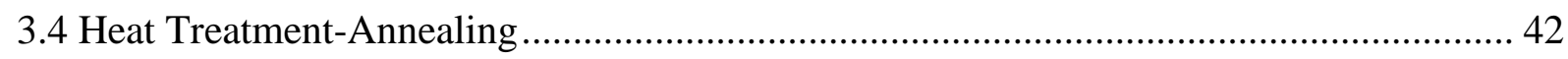

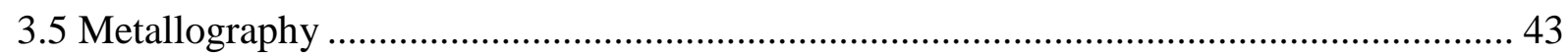

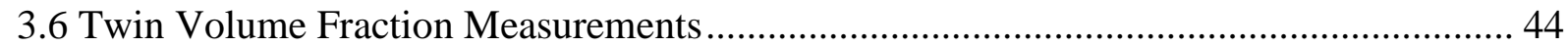

3.7 Quantitative Texture Analysis............................................................................................ 45

Chapter 4 Twinning and Texture Development during Compression .................................... 51

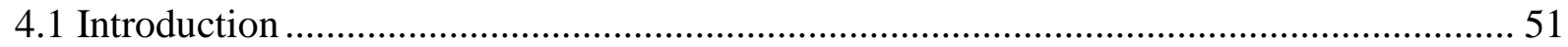

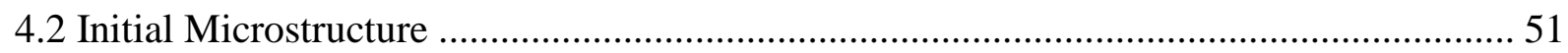

4.3 Twin Growth Behavior …………………………................................................. 52

4.4 Role of Twinning on Strain Hardening .......................................................................... 58

4.5 Texture Transformation during Compressive Strain.......................................................... 62

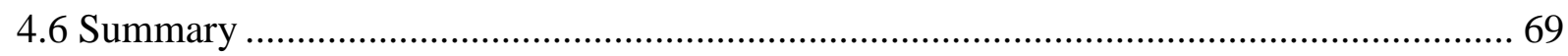

Chapter 5 Effect of Crystallographic Orientation and Loading Direction on Schmid

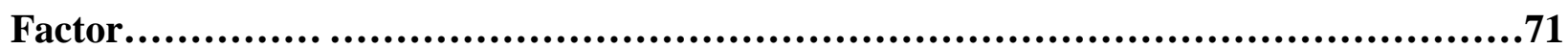

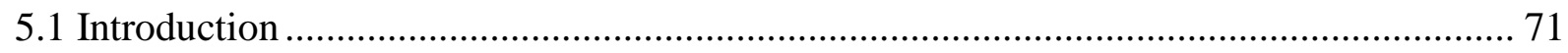

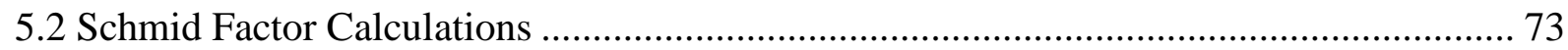

5.3 Influence of Loading Direction and Basal Plane Inclination on Schmid Factor................ 77

5.4 Effect of In-plane Loading Direction on Compressive Behavior ........................................ 84

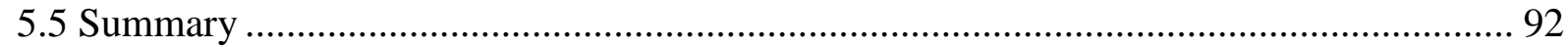

Chapter 6 Role of Pre-compressive Deformation on Twinning, Texture and Hardening Behavior............................................................................................................... 94 


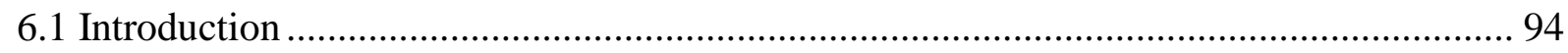

6.2 Effect of Loading Direction on Compressive Properties ................................................... 95

6.3 Effect of Pre-Strain and Loading Direction on Compressive Properties ........................... 96

6.4 Effect of Pre-strain and Loading Direction on Microstructure ........................................ 110

6.5 Effect of Pre-strain and Loading Direction on Texture Evolution..................................... 119

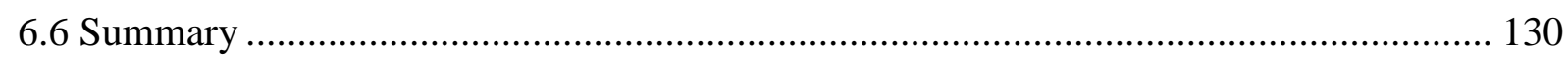

Chapter 7 Influence of Pre-deformation and Subsequent Annealing on Strain Hardening and Anisotropy ................................................................................................................ 134

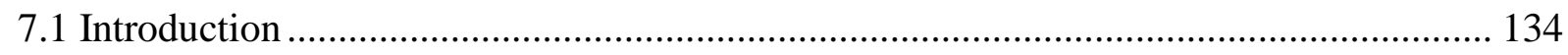

7.2 Effect of Pre-Strain and Subsequent Annealing on Compressive Properties .................. 136

7.3 Effect of Pre-Strain and Annealing on Microstructure Development............................... 145

7.4 Recrystallization Kinetics ............................................................................................ 147

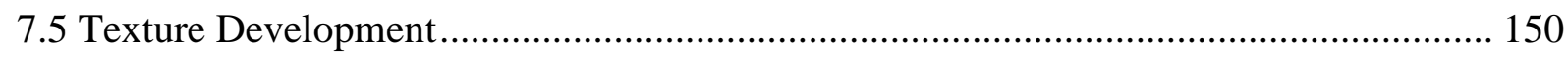

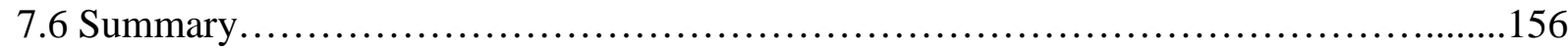

Chapter 8 Conclusions and Future Work ......................................................................... 158

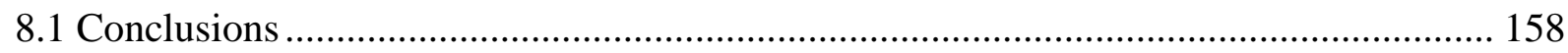

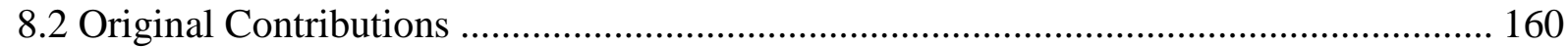

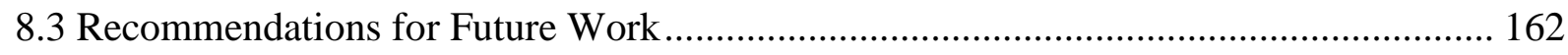

Appendix A Calculation of Schmid Factor........................................................................................ 165

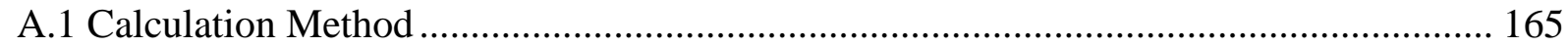

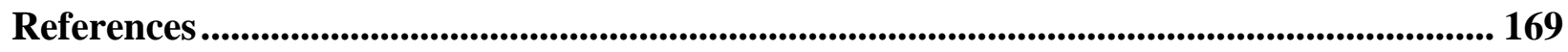




\section{LIST OF TABLES}

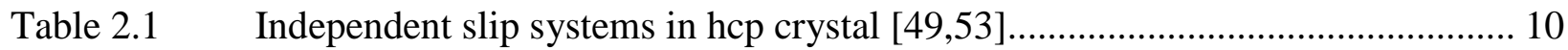

Table 2.2 Description of the $\{10 \overline{1} 2\}$ and $\{10 \overline{1} 1\}$ twinning invariants [77] ..................... 16

Table 3.1 Chemical composition (wt \%) of the extruded AM30 magnesium alloy............ 38

Table 3.2 Temperature and time selected during annealing......................................... 42

Table 4.1 Main texture components identified from Euler angles ................................... 64

Table 5.1 A comparison of SF values for all the extension twin variants using different methods at a $0^{\circ}$ inclination angle and compression along the

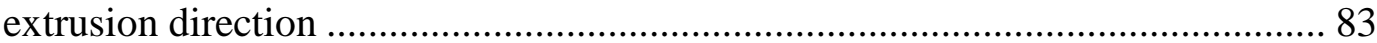




\section{LIST OF FIGURES}

Figure 2.1: (a) Hexagonal close-packed (hcp) crystal structure, (b) possible slip directions [46]

Figure 2.2: (a) Basal- $<\boldsymbol{a}>$, (b) Prismatic- $<\boldsymbol{a}>$, (c) Pyramidal- $<\boldsymbol{a}>$, and (d) Pyramidal$<\boldsymbol{c}+\boldsymbol{a}>$ slip systems in magnesium [42,54]

Figure 2.3: Schematic diagram showing how twinning results from an applied shear stress $\tau$, (b) open circles represent atoms that did not change position; dashed and solid circles represent original and final atom positions, respectively $[55,56]$.

Figure 2.4: $\quad$ For a single crystal subjected to a shear stress $\tau$, (a) deformation by slip; (b) deformation by twinning [55]

Figure 2.5: (a) Schematic illustration of extension twins $\{10 \overline{1} 2\}<\overline{1} 011>$ and, (b) contraction twins $\{10 \overline{1} 1\}<\overline{1} 012>$ in magnesium, where the corresponding shear direction are indicated

Figure 2.6: Twinning elements $K_{1}, K_{2}, \eta_{1}$ and $\eta_{2}$, shown in relation to the plane of shear [78] 16

Figure 2.7: $\quad$ (a) Twinning elements of twinning mode $\{10 \overline{1} 2\}<10 \overline{1} 1>$, (b) schematic image of twin mechanism. Extension twin occurs when a compressive load is applied perpendicular to the $c$-axis $[49,79]$.

Figure 2.8: Influence of deformation temperature on the critical resolved shear stress [87]

Figure 2.9: Relationship between the resolved shear stress acting on the deformation system and the force applied externally on the single-crystal rod [55] 24

Figure 2.10: Schematic diagram showing the (a) extrusion process, and (b) the formation of basal texture in extruded magnesium [93]. 25

Figure 3.1: $\quad$ Sample dimension for the compression test prepared according to ASTM E9-09 standards for, (a) cylindrical sample, (b) schematic diagram showing the orientation of samples with respect to the extrusion direction (ED). 38

Figure 3.2: $\quad$ Rectangular sample dimension for the compression test 39 
Figure 3.3: Schematic diagram showing the variables, temperature and time during annealing......

Figure 3.4: An example showing how to evaluate the volume fraction of twins via a point counting technique. In this example the volume fraction is obtained to be $182 / 357=51 \%$.

Figure 3.5: Schematic illustration of texture measurements using X'pert MRD system......... 46

Figure 3.6: $\quad$ (a) Definition of pole direction angles $\phi$ and $\Psi$, (b) stereographic projection in a pole figure [90]

Figure 3.7: Schematic representation of a microstructure consisting of different grains $i$ of volume $V_{i}$ with different orientations. Similar orientations $g$ within an orientation range $d g$ is color coded with the same gray value [90]

Figure 4.1: (a) Microstructure, (b) (0001) and (1010) pole figures of the extruded AM30 magnesium alloy, (c) schematic diagrams illustrating the relationship between the $c$-axis of most grains and the compression axis oriented horizontally ...... 53

Figure 4.2: (a) Initial twin-free microstructure and twin growth during stepwise compression after a cumulative true strain amount of (b) $1.7 \%$, (c) $4.4 \%$, and (d) $9.9 \%$ along the ED 55

Figure 4.3: Twin width vs. cumulative true strain after stepwise compression 56

Figure 4.4: Schematic illustration of the growth of $\{1012\}$ extension twins in a grain: (a) formation of twins inside a grain by rotating basal planes at an angle of $86.3^{\circ}$, (b) twin growth, (c) coalescence of twins (or vanishing of twin boundaries) to continue twin growth

Figure 4.5: (a) Compressive true stress-true strain curves of extruded AM30 magnesium alloy deformed to different strain levels, and (b) strain hardening rate as a function of true strain.

Figure 4.6: Optical micrographs at different strain levels of, (a) $1.5 \%$, (b) $2.4 \%$, (c) 4.3\%, (d) $8.4 \%$ and (e) $12.9 \%$ during compression of extruded AM30 magnesium alloy 
Figure 4.7: (0001) and (1010) pole figures obtained from the compressed samples at a strain amount of (a) 1.5\%, (b) 4.3\%, (c) $8.4 \%$, and (d) $12.9 \%$.

Figure 4.8: (a) ODF sections at $\varphi_{2}=0^{\circ}$ and $\varphi_{2}=30^{\circ}$, from the samples strained at $0 \%$, $4.3 \%$ and $12.9 \%$, and (b) the change of intensity of main texture components with the strain . 65

Figure 4.9: Texture volume fraction vs. strain after compression..................................... 68

Figure 5.1: (a) Schematic presentation of $\{10 \overline{1} 2\}$ extension and $\{10 \overline{1} 1\}$ contraction twins in magnesium crystal system, (b) considering a basal plane inclination angle $\beta$, with respect to the extrusion direction (ED) 75

Figure 5.2: $\quad$ Illustration of the hcp crystal structure of magnesium to calculate Schmid factor using the relationship between the angle of compressive loading, twin plane and twin plane normal direction. The shaded FEON is an example of $\{10 \overline{1} 2\}$ extension twin variant 76

Figure 5.3: Schmid factors of $\{10 \overline{1} 2\}$ extension twins as a function of loading axis with the extrusion direction at different $\beta$ angles of (a) $0^{\circ}$, (b) $15^{\circ}$, (c) $30^{\circ}$, and (d) $45^{\circ}$ 80

Figure 5.4: Schmid factors as a function of loading axis $(\alpha)$ and basal plane inclination

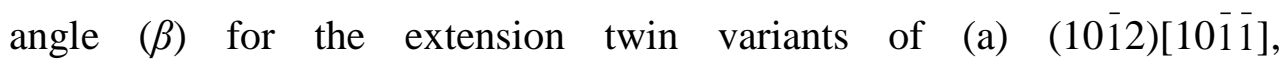
(b) $(1 \overline{1} 02)[1 \overline{1} 0 \overline{1}]$, and (c) $(01 \overline{1} 2)[01 \overline{1} \overline{1}]$ 82

Figure 5.5: (a) Compressive true stress-true strain curves, (b) yield strength (YS) and ultimate compressive strength (UCS) and (c) fracture strain as a function of sample orientation, (d) strain hardening rate as a function of true strain, and (e) the slope of stage B in the strain hardening rate vs. sample orientation in the extruded AM30 magnesium alloy.... 87

Figure 5.6: Typical microstructure observed at a strain level of $4.3 \%$ in samples oriented at an angle of (a) $0^{\circ}$, (b) $45^{\circ}$ and (c) $90^{\circ}$ relative to the ED. 89 
Figure 5.7: Pole figures obtained from the samples oriented at an angle of (a) $45^{\circ}$ and (b) $90^{\circ}$ from the ED and compressed at a strain amount of $4.3 \%$, where CD stands for the compression direction

Figure 6.1: $\quad$ True stress-true strain curves of an extruded AM30 along the ED, TD and ND. 96

Figure 6.2: (a, b, c) True stress-true strain curves obtained in re-compression along the ED, TD and ND, respectively, after pre-compression at various strain levels along the ED 99

Figure 6.3: Effect of pre-strain along the ED on (a) compressive yield strength (YS), (b) ultimate compressive strength (UCS), and (c) hardening capacity of recompressed samples along the ED, TD and ND

Figure 6.4: $\quad$ For the ED-ED and ED-TD compressed samples, $(\mathrm{a}, \mathrm{b})$ strain hardening rate vs. true strain respectively, (c) change range of strain hardening rate in stage $\mathrm{B}\left(\Delta \theta_{\mathrm{B}}\right)$ as a function of pre-strain. For the ED-ND compressed samples, (d) strain hardening rate vs. true strain, (e) peak value of strain hardening rate, $\theta_{\max }$ vs. pre-strain, (f) $\log (d \theta / d \varepsilon)$ vs. Log (pre-strain).

Figure 6.5: Interior microstructures of the AM30 samples subjected to a continuous compressive strain of (a) $4.0 \%$ and (b) $8.2 \%$ along the ED at a strain rate of $1.25 \times 10^{-4} \mathrm{~s}^{-1}$ and room temperature

Figure 6.6: Interior microstructures of the AM30 samples subjected to a two-step compressive deformation, (a) 4.0\% re-compression along the ED and (b) $4.0 \%$ re-compression along the TD, after a $4.0 \%$ pre-strain along the ED.

Figure 6.7: Change of microstructures on the pre-polished surface of an AM30 sample subjected to (a) $4.0 \%$ pre-strain along the ED only, and (b) $4.0 \%$ pre-strain along the ED plus $4.0 \%$ re-compression along the TD, where the red dashed ovals denote de-twinning or twin shrinkage (narrowing, shortening), and the blue arrows indicate the formation of new twins 
Figure 6.8: $\quad$ Microstructures of the compressed AM30 samples at a strain of (a) 1.7\% along ED, (b) 3.7\% re-compression along ND after 2.1\% pre-strain along ED, (c) $4.0 \%$ along ED, (d) 3.7\% re-compression along ND after 3.7\% prestrain along ED, (e) $8.2 \%$ along ED, (f) $3.7 \%$ re-compression along ND after $7.9 \%$ pre-strain along ED

Figure 6.9: (a) Initial microstructure and twin width during stepwise compression after a cumulative strain amount of (b) 3.7\%ED, (c) 3.7\%ED-3.5\%ND, and (d) 3.7\%ED-7.7\% ND

Figure 6.10: Twin width vs. cumulative true strain along the ND of pre-strained sample...... 118

Figure 6.11: (0001) and (1010) pole figures of (a) 4.0\%ED-4.0\%ED, and (b) 4.0\%ED4.0\%TD compressed samples, where " 1 " stands for the pre-compression direction along the ED, "2" denotes the re-compression direction, $\mathrm{RD}$ signifies the radial direction of the round samples, and ED, TD, ND indicate the extrusion direction, transverse direction and normal direction of extruded plate, respectively

Figure 6.12: (0001) and (1010) pole figures of (a) 4.0\%ND, (b) 2.1\%ED-3.7\%ND, (c) 3.7\%ED-3.7\%ND, (d) 7.9\%ED-3.7\%ND

Figure 6.13: The change of intensity of main texture components with cumulative true strain.

Figure 6.14: Schematic illustration of $\{10 \overline{1} 2\}$ extension twinning in a grain: (a) formation of a twin inside a grain by rotating an angle of $86.3^{\circ}$ of basal planes, (b) twin growth, through the nucleation and glide of twinning dislocations on the twin boundaries, (c) twin narrowing/shrinkage through the nucleation and glide of twinning dislocations with a reverse sign, (d) double (or secondary) twinning inside the pre-existing parent twin, with the same twin variant as the parent twin 
Figure 7.1: $\quad$ Typical true stress-true strain curves of extruded AM30 with and without a $4.3 \%$ pre-strain along the ED

Figure 7.2: $\quad$ (a) Typical true stress-true strain curves of $4.3 \%$ pre-strained and annealed samples at $250^{\circ} \mathrm{C}, 350^{\circ} \mathrm{C}$ and $450^{\circ} \mathrm{C}$ for $3 \mathrm{~h}$, (b) compressive yield strength (CYS), and (c) ultimate compressive strength (UCS) as a function of annealing temperature

Figure 7.3: $\quad$ Typical true stress-true strain curves of $4.3 \%$ pre-strained and annealed samples at $450^{\circ} \mathrm{C}$ for different times of $3 \mathrm{~h}, 6 \mathrm{~h}$ and $12 \mathrm{~h}$

Figure 7.4: $\quad$ Strain hardening rate vs. true strain of $4.3 \%$ pre-strained and annealed AM30 alloy at (a) $250^{\circ} \mathrm{C}, 350^{\circ} \mathrm{C}$ and $450^{\circ} \mathrm{C}$ for $3 \mathrm{~h}$, (b) $450^{\circ} \mathrm{C}$ for $3 \mathrm{~h}, 6 \mathrm{~h}$ and $12 \mathrm{~h}$. 142

Figure 7.5: $\quad$ Stage B hardening behavior of 4.3\% pre-compressed and annealed AM30 Mg alloy, (a) the extent of stage $\mathrm{B}\left(\Delta \theta_{\mathrm{B}}\right)$ and (b) the slope of stage $\mathrm{B}$ as a function of annealing temperature while keeping a constant annealing time of 3h, (c) the slope of stage B as a function of annealing time while keeping a given annealing temperature of $450^{\circ} \mathrm{C}$

Figure 7.6: Volume fraction of extension twins vs. annealing time at a constant annealing temperature of $450^{\circ} \mathrm{C}$ for the $4.3 \%$ pre-strained samples 146

Figure 7.7: $\quad$ (a) Recrystallization fraction $\left(X_{r}\right)$ vs. time and (b) JMAK plot showing the recrystallization kinetics of the $4.3 \%$ pre-strained AM30 alloy during annealing at $450^{\circ} \mathrm{C}$ using a metallographic observation method 148

Figure 7.8: (0001) and (1010) pole figures of the $4.3 \%$ pre-compressed and subsequently annealed samples at $450^{\circ} \mathrm{C}$ for (a) $3 \mathrm{~h}$ and (b) $12 \mathrm{~h}$, 
respectively, where ED indicates the extrusion direction and $\mathrm{RD}$ stands for the radial direction

Figure 7.9: $\quad$ Microstructure of the $4.3 \%$ re-compressed sample after annealing of $4.3 \%$ pre-strained samples at $450^{\circ} \mathrm{C}$ for (a) $3 \mathrm{~h}$ and (b) $12 \mathrm{~h}$, respectively.....

Figure 7.10: (0001) and (1010) pole figures of the $4.3 \%$ re-compressed sample after annealing of $4.3 \%$ pre-strained samples at $450^{\circ} \mathrm{C}$ for (a) $3 \mathrm{~h}$ and (b) $12 \mathrm{~h}$, respectively

Figure 7.11: Schematic illustration of recrystallization process during annealing of a compressed AM30 alloy: (a) the beginning of recrystallization with preferential nucleation in twins and grains, (b) formation of new small grains within twins and at grain boundaries, (c) recrystallized grains 155

Appendix A1: (a-d) Some sectional views of Fig. 5.2 used to calculate Schmid Factor 165 


\section{LIST OF APPENDICES}

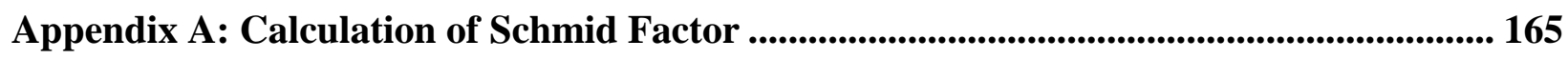

A.1 Calculation Method ...................................................................................... 165 
NOMENCLATURE

\begin{tabular}{|c|c|}
\hline$a, c$ & Lattice parameter \\
\hline$\tau$ & Shear stress \\
\hline CRSS & Critical Resolved Shear Stress \\
\hline$K_{1}$ & Twin plane \\
\hline$K_{2}$ & Conjugate twin plane \\
\hline$\eta_{1}$ & Shear direction \\
\hline$\eta_{2}$ & Conjugate shear direction \\
\hline$S$ & Shear \\
\hline$h, k, i, l$ & Miller indices \\
\hline$\sigma$ & Stress \\
\hline$\varepsilon$ & Strain \\
\hline$\tau_{c}$ & Critical shear stress \\
\hline$\varphi$ & $\begin{array}{l}\text { Angle between the loading direction and } \\
\text { twinning direction }\end{array}$ \\
\hline$\lambda$ & $\begin{array}{l}\text { Angle between the loading and twin normal } \\
\text { direction }\end{array}$ \\
\hline$\phi, \Psi$ & Rotation axes in goniometer \\
\hline $2 \theta$ & Bragg angle \\
\hline$f(g)$ & Texture orientation intensity \\
\hline$O D F$ & Orientation Distribution Function \\
\hline$M R D$ & Multiples of Random Distributions \\
\hline
\end{tabular}




\begin{tabular}{|c|c|}
\hline$f_{x}$ & Texture volume fraction \\
\hline$\varphi_{1} \Phi \varphi_{2}$ & Euler angles \\
\hline$M_{0}$ & Initial orientation density \\
\hline$M_{x}$ & Orientation density at strain, $x$ \\
\hline$M_{f}$ & Final orientation density \\
\hline$S F$ & Schmid Factor \\
\hline$\alpha$ & Loading angle \\
\hline b & Tilt angle \\
\hline UCS & Ultimate Compressive Strength \\
\hline$Y S$ & Yield stress \\
\hline$H_{c}$ & Hardening capacity \\
\hline$d \theta / d \varepsilon$ & Slope of strain hardening rate \\
\hline$E D$ & Extrusion direction \\
\hline$T D$ & Transverse direction \\
\hline$N D$ & Normal direction \\
\hline TDs & Twinning dislocations \\
\hline$V_{f}$ & Twin volume fraction \\
\hline$V_{f 0}$ & Twin volume fraction after pre-compression \\
\hline$V_{f t}$ & $\begin{array}{l}\text { Twin volume fraction after pre-compression and } \\
\text { annealing }\end{array}$ \\
\hline$T$ & Time \\
\hline$X_{r}$ & Fraction of recrystallization \\
\hline$J M A K$ & Johnson-Mehl-Avrami-Kolmogorov \\
\hline
\end{tabular}




\section{CHAPTER 1}

\section{Introduction}

\subsection{Introduction and Motivation}

During the early years of the twenty-first century, the efficiency of energy consumption has become increasingly important, and the demand for energy has increased tremendously, while at the same time oil’s “tipping point” has passed, based upon the report in a recent Nature article [1]. Although renewable sources of energy are still under intense investigation, advances in materials and structural design make it possible to expend less energy for the same duty. This is particularly true in the transportation industries, e.g., for ground and aerospace vehicles, where the application of lighter and more durable materials becomes important since light weighting is today considered one of the salient strategies for reducing fuel consumption and anthropogenic environment-damaging, climate-changing, human death-causing ${ }^{1}$ and high-priced emissions [27]. It has been reported that the fuel efficiency of passenger vehicles can be enhanced by $6 \sim 8 \%$ for each $10 \%$ reduction in weight [8-10]. Light metals, such as aluminum and titanium alloys, have been successfully used in the aerospace and automotive sectors for structural applications. As ultra-lightweight strategic materials, magnesium alloys have recently kindled considerable interest in the automotive and aerospace industries, and are being intensively studied for potential load-bearing structural applications to reduce vehicle weight and increase fuel

\footnotetext{
${ }^{1}$ According to Science News in an article entitled “Air pollution kills 7 million people a year” on March 25, 2014 at http://news.sciencemag.org/signal-noise/2014/03/air-pollution-kills-7-million-people-year: “Air pollution isn’t just harming Earth; it's hurting us, too. Startling new numbers released by the World Health Organization today reveal that one in eight deaths are a result of exposure to air pollution. The data reveal a strong link between the tiny particles that we breathe into our lungs and the illnesses they can lead to, including stroke, heart attack, lung cancer, and chronic obstructive pulmonary disease."
} 
efficiency [2] due to their high strength-to-weight ratio, dimensional stability, and good machinability and recyclability. Wrought (extruded, rolled, etc.) magnesium alloys have in general better tensile properties [11,12] and fatigue resistance [13-19] compared with cast magnesium alloys [20-21].

The application of wrought magnesium alloys requires comprehensive knowledge about the deformation behavior of these materials and their final performance under service loading. Magnesium alloys, having a hexagonal close-packed (hcp) crystal structure, show a pronounced anisotropy in their mechanical properties [22-25]. The plastic anisotropy of magnesium alloy is greatly influenced by the preferred orientation (more commonly called "crystallographic texture”, or just texture) formed in wrought alloys. Generally, the wrought magnesium alloys form a strong basal texture with most basal planes aligned parallel to the process direction (i.e., extrusion or rolling direction) [22-30], and exhibit a high degree of anisotropy and poor room temperature formability due to the limited slip systems and the polar characteristics of deformation twinning.

The occurrence of deformation twinning in magnesium alloys has been widely reported in the literature [30-34]. However, its precise influence on hardening behavior and texture development under monotonic and cyclic loading has not yet been well understood. In $\mathrm{Mg}$ alloys, the variations in the strain hardening behavior can also be ascribed to the influence of the initial texture on slip and twinning. Previous research has indicated that the mechanical response and the evolution of texture during straining are significantly affected by the interaction between the strain path and the initial texture. The directionality of the twinning systems in turn leads to strong anisotropic mechanical behavior in the textured magnesium alloys [35-41]. 
Recrystallization has been reported to be an effective technique in plastically deformed magnesium alloys to produce nucleation sites within twins and untwinned regions with modified grain size, shape or texture. This opens up the possibility of controlling the mechanical response by designing crystallographic textures appropriate to particular strain paths. The studies on the close relationships between the crystallographic texture and plastic deformation mechanisms in wrought magnesium alloys, which reciprocally influence each other, provide valuable information for the general understanding of the plastic deformation of polycrystalline materials with hcp structures, and are beneficial for designing optimal alloying and forming schemes to fabricate such wrought magnesium alloys.

The research presented here is about the fundamental study of the mechanical behavior and deformation mechanisms in an extruded magnesium alloy, with a particular focus on twinning and texture development. The major objectives of the present research are to identify the role of deformation twinning (as one of the deformation mechanisms) on the plastic deformation behavior of extruded magnesium alloys, and its correlation with microstructure and textures, based on a fundamental understanding of the mechanical properties under various loading conditions and sample orientations using X-ray diffraction as a major characterization tool. The work constitutes a benchmark for understanding the effects of the plastic deformation mechanisms, such as twinning, de-twinning and dislocation slip on the mechanical behavior in wrought magnesium alloys. 


\subsection{Objectives of the Research}

The research was aimed at investigating the deformation behavior and texture development of an extruded magnesium alloy under uniaxial compression loading. By determining the mechanical properties of the material under a series of varying loading conditions, the research project involved identifying the effects of (i) sample orientation, (ii) multiple loading direction, and (iii) static recrystallization on the texture development and deformation mechanisms of extruded $\mathrm{Mg}$ alloys. The following studies were conducted and the specific aims of each study are listed below:

\section{Study 1: Effects of Sample Orientation}

The effect of sample orientation on the deformation behavior and texture development during uniaxial compression was investigated with the following objectives:

i. To identify if the sample orientation would affect the flow stress behavior and texture development.

ii. To investigate the flow behavior of extruded AM30 alloy subjected to compressive loading at various strain levels and the successive change of texture components.

iii. To ascertain if differently oriented samples with extrusion direction under various loading parameters, such as, strain levels, and strain rates would affect deformation behavior and texture development. 
iv. To propose a geometric model using hexagonal close packed crystal structure for calculating the Schmid Factor values which could be used to select active twin variants during deformation.

v. Verification of the calculated Schmid Factor values considering different crystallographic orientations and loading directions with the experimental data obtained after compression test.

\section{Study 2: Effect of Pre-compressive Deformation}

The effect of pre-compressive deformation on microstructure and texture development during uniaxial compression was investigated under different loading parameters to obtain the following objectives:

i. To identify the influence of twins introduced by pre-straining on the subsequent texture and strain hardening upon re-loading with and without changing the loading direction.

ii. To do compression tests with cylindrical and rectangular shaped samples with varying loading directions to reveal the degree of anisotropy.

iii. To show the effect of pre-strain amount on twinning, de-twinning and the associated texture change. 


\section{Study 3: Static Recrystallization}

This study was aimed at investigating the potential twin disappearance during annealing and the effect of pre-strain on recrystallization of extruded Mg alloys. The deformation mechanism and twin formation or disappearance after subsequent annealing was investigated. Then further deformation was conducted to reveal the potential change and difference in the twin formation or deformation behavior after the high temperature exposure. Specially, the study aimed to do the following:

i. To observe the change of volume fraction of twins as a function of annealing temperature and time of compression strain induced (pre-strain) samples. The response of twin volume fraction during annealing was correlated with the JMAK model to examine static recrystallization phenomena.

ii. To do re-compression of some selected samples after annealing so as to examine the effect of annealing or thermal exposure on the further twin formation or deformation behavior.

iii. To measure the change of orientation, texture analysis was also performed after annealing of pre-compressed samples. The intensity variation of texture was compared with the pre-deformed and annealed sample as well as after re-compression.

This thesis has been structured as follows:

Chapter-2 presents a literature survey that contains a concise review of magnesium material characteristics, deformation modes and the factors affecting the activity of deformation. 
Chapter-3 presents the detailed experimental methodology to design samples, to conduct the experiments, heat treatment parameters, and material characterization by metallography and texture analysis.

Chapter-4 examines the influence of compressive strain on twin formation, their growth, hardening behavior and texture development.

Chapter-5 presents a model proposed to calculate the Schmid factor at different crystallographic orientations and loading directions and the validation of the model using the experimental data.

Chapter-6 describes the influence of twins introduced by pre-straining on the subsequent twinning, de-twinning, texture development and strain hardening upon re-loading with and without changing the loading orientation.

Chapter-7 shows the role of twinning introduced by pre-compression on static recrystallization during annealing and texture evolution upon reloading of annealed samples.

Chapter-8 summarizes the outcomes from the study and makes concluding remarks as well as suggesting directions for the future work. 


\section{CHAPTER 2}

\section{Literature Review}

\subsection{Magnesium: Material Characteristics}

In 1808, Magnesium was discovered and isolated by Sir Humphrey Davy, but it took about 100 years for the development of real demand [42]. Magnesium is the lightest structural metal available in the world with a density of $1.74 \mathrm{~g} / \mathrm{cm}^{3}, 35.6 \%$ lower than that of aluminum and 61.3\% lighter than titanium [43]. The attraction of magnesium as an engineering material is its combined properties of light weight, high strength-to-weight ratio and high specific stiffness at both ambient and elevated temperatures. However, the use of magnesium as a structural material has been limited. The bulk of magnesium was used as an alloying element in aluminum alloys [43]. Since 1993 there has been renewed interest in using magnesium-based alloys in automobile, aerospace and other household and sport applications [42]. As Bettles et al. [44] reported, the interest in magnesium alloys has been revived since the turn of the millennium, and the alloy is considered common today. The keen interest in the research and development (R\&D) of magnesium alloys in the past decade or so could perhaps be demonstrated by the high citation number $\left(3059^{2}\right)$ of the paper entitled “Magnesium: Properties - applications - potential” authored by Mordike and Ebert since it was published in 2001 [45]. The challenges in using wrought magnesium for automotive applications lie in cost as well as in properties. Major shortcomings of the magnesium alloys in the wrought condition are poor formability, basal texture, and tension-compression yield asymmetry associated with twinning, low ductility, etc.

\footnotetext{
${ }^{2}$ Based on a Google search on August 28, 2014.
} 


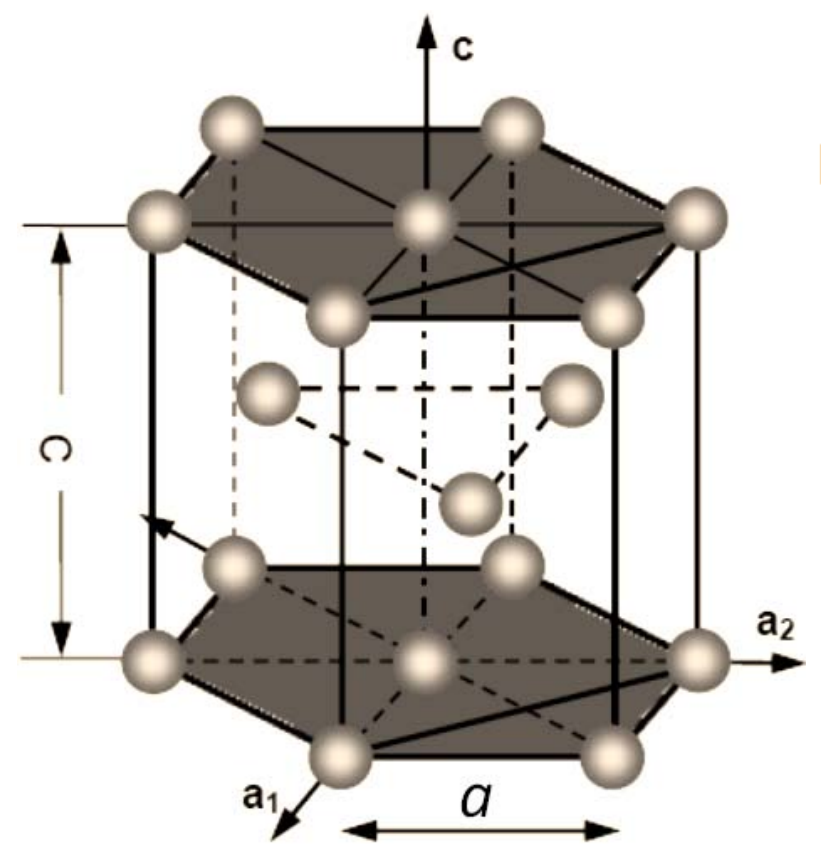

(a)

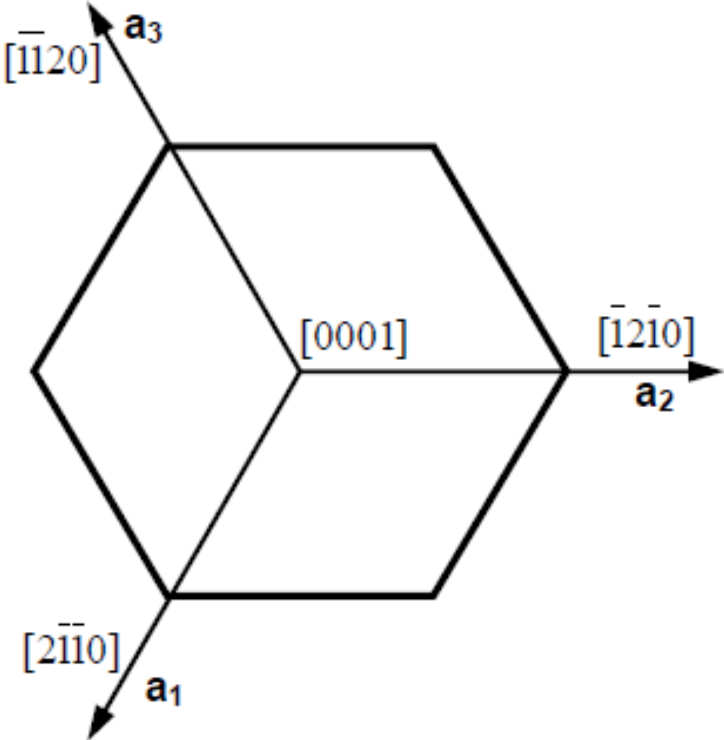

(b)

Figure 2.1: (a) Hexagonal close-packed (hcp) crystal structure, (b) possible slip directions [46].

Magnesium has a hexagonal close packed (hcp) crystal structure, as shown in Fig. 2.1(a), where the positions of the atoms and possible slip directions are shown in Fig. 2.1(a) and (b) respectively. The cell geometry is described by four axes. Three of the axes are coplanar with $\mathbf{a}_{1}$, $\mathbf{a}_{2}$ and $\mathbf{a}_{3}$ oriented $120^{\circ}$ to one another. The fourth axis, referred to as the $c$-axis, is perpendicular to the basal plane containing $\mathbf{a}_{1}, \mathbf{a}_{2}$ and $\mathbf{a}_{3}$. In the hcp unit cell the lengths $\mathbf{a}_{1}, \mathbf{a}_{2}$ and $\mathbf{a}_{3}$ are equal. One of the important material lattice parameters which determine the deformation behavior is the $c / a$ ratio, where $a$ is the interatomic distance in the basal plane along any of the three $<11 \overline{2}$ $0>$ directions (Fig. 2.1(b)), and $c$ is the distance between the first and the third layers. The lattice parameters of pure magnesium at room temperature are: $a=0.32092 \mathrm{~nm}$ and $c=0.52105 \mathrm{~nm}$ [47]. The c/a ratio is 1.6236 which is close to the ideal value of 1.633 . However, no pure metal has this ideal $c / a$ ratio. Depending on the $c / a$ ratio, some metals, such as $\mathrm{Zr}$, and $\mathrm{Cd}$ show twinning dominant deformation when a compressive load is applied along the $\boldsymbol{c}$ axis, while a 
tensile load along the $\boldsymbol{c}$ axis is needed for twinning in $\mathrm{Mg}$ and Be. Twin formation in hcp materials strongly depends on the c/a ratio which will be discussed later.

\subsection{Deformation Systems in Magnesium}

\subsubsection{Dislocation-Slip Systems}

In a polycrystalline material, to accommodate deformation, five independent slip systems have to be activated $[48,49]$. In Mg, three possible dislocation Burgers vectors can be active on various slip planes: $\langle\mathbf{a}>=1 / 3<11 \overline{2} 0>$ (on basal or prismatic planes), $\langle\mathbf{c}>=1 / 3<11 \overline{2} 0>$ (on prismatic planes), and $<\mathbf{c}+\mathbf{a}>=1 / 3<11 \overline{2} \overline{3}>$ (on the $\{11 \overline{2} 2\}$ pyramidal plane) [47,50], as shown in Fig. 2.2. The important slip systems in magnesium are summarized in Table 2.1. Transmission Electron Microscopy (TEM) studies in the 1970's further confirmed the existence of these systems and the dislocations involved [51,52].

Table 2.1: Independent slip systems in hcp crystal [49,53].

\begin{tabular}{|c|c|c|c|}
\hline Slip system & Direction & $\begin{array}{c}\text { Crystallographic } \\
\text { components }\end{array}$ & $\begin{array}{c}\text { No. of } \\
\text { independent } \\
\text { modes }\end{array}$ \\
\hline Basal slip & $\mathbf{a}$ & $\{0001\}<11 \overline{2} 0>$ & 2 \\
\hline Prismatic slip & $\mathbf{a}$ & $\{10 \overline{1} 0\}<11 \overline{2} 0>$ & 2 \\
\hline 1st-order Pyramidal slip & $\mathbf{a}$ & $\{10 \overline{1} 1\}<11 \overline{2} 0>$ & 4 \\
\hline 2nd-order Pyramidal slip & $\mathbf{c}+\mathbf{a}$ & $\{11 \overline{2} 2\}<11 \overline{2} 3>$ & 5 \\
\hline
\end{tabular}



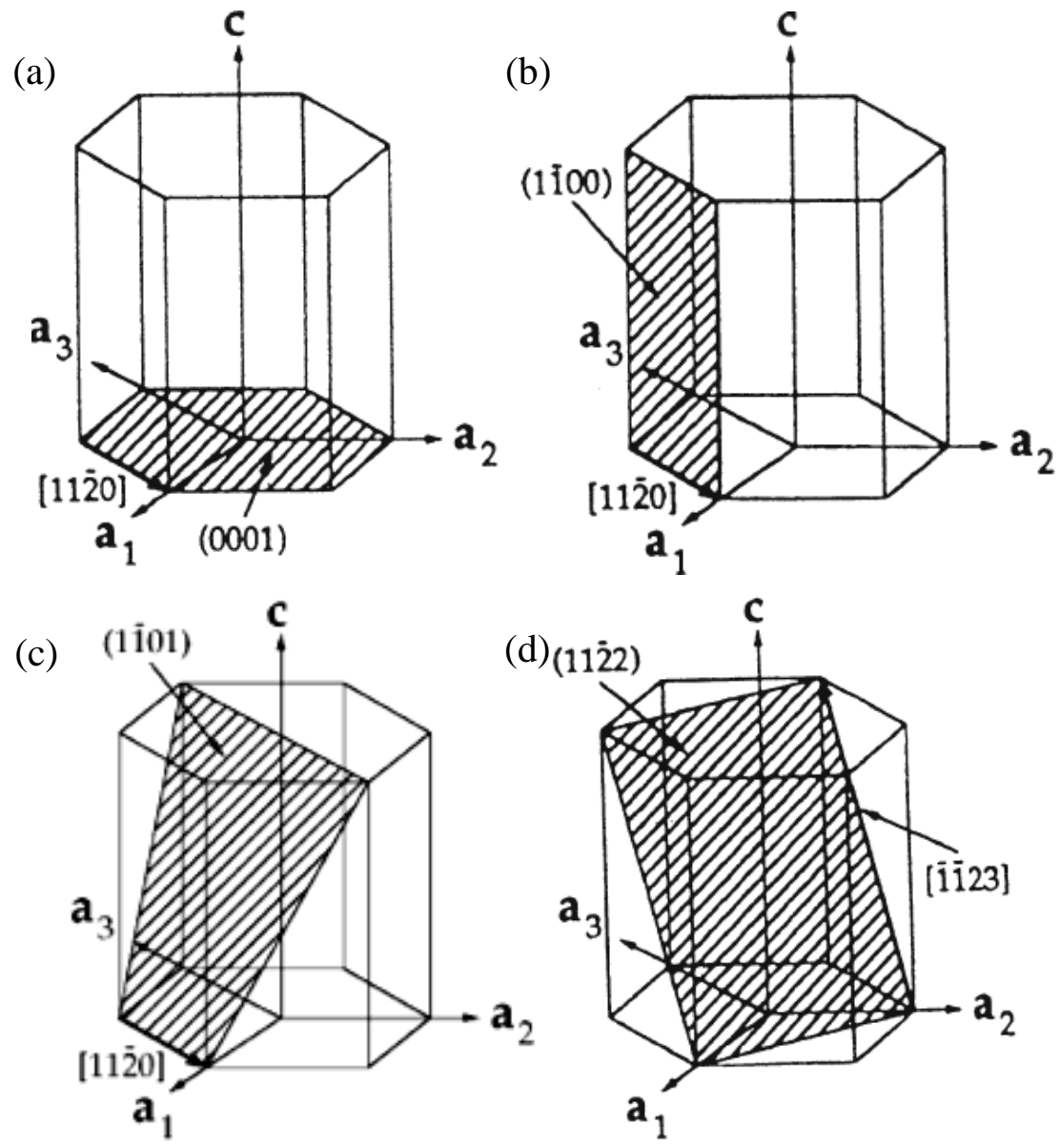

Figure 2.2: (a) Basal- $<\boldsymbol{a}>$, (b) Prismatic- $<\boldsymbol{a}>$, (c) Pyramidal- $<\boldsymbol{a}>$, and (d) Pyramidal- $<\boldsymbol{c}+\boldsymbol{a}>$ slip systems in magnesium [42,54].

Among the four dominant slip systems listed in Table 2.1, three of them (basal, prismatic and lst-order pyramidal slip) involve the slip of dislocations with $<11 \overline{2} 0>$ (or $<\mathbf{a}>$ ) type Burgers vectors. With reference to the Von Mises criterion, basal and prismatic slip can only offer two independent slip modes in each case. While the lst-order pyramidal slip can offer four independent slip modes, this is still short of the required five for homogeneous deformation. Only the 2nd-order pyramidal slip, with its non-basal Burger vectors (or $<\mathbf{c}+\mathbf{a}>$ ), will 
accommodate deformation along the $c$-axis and meet the requirement for five independent slip modes. However, this type of dislocation slip does not normally occur at room temperature.

\subsubsection{Twinning}

The twinning phenomenon has been well described by Dieter [56] as follows: “Twinning results when a portion of the crystal takes up an orientation that is related to the orientation of the rest of the untwined lattice in a definite, symmetrical way". Then the twinned portion of the crystal would be a mirror image of the parent crystal. The plane of symmetry between the two portions is known as the twinning plane. The typical atomic arrangement of twinning is illustrated in Fig.

2.3. The crystal will twin about the twinning plane when a shear stress is applied (Fig. 2.3(b)).

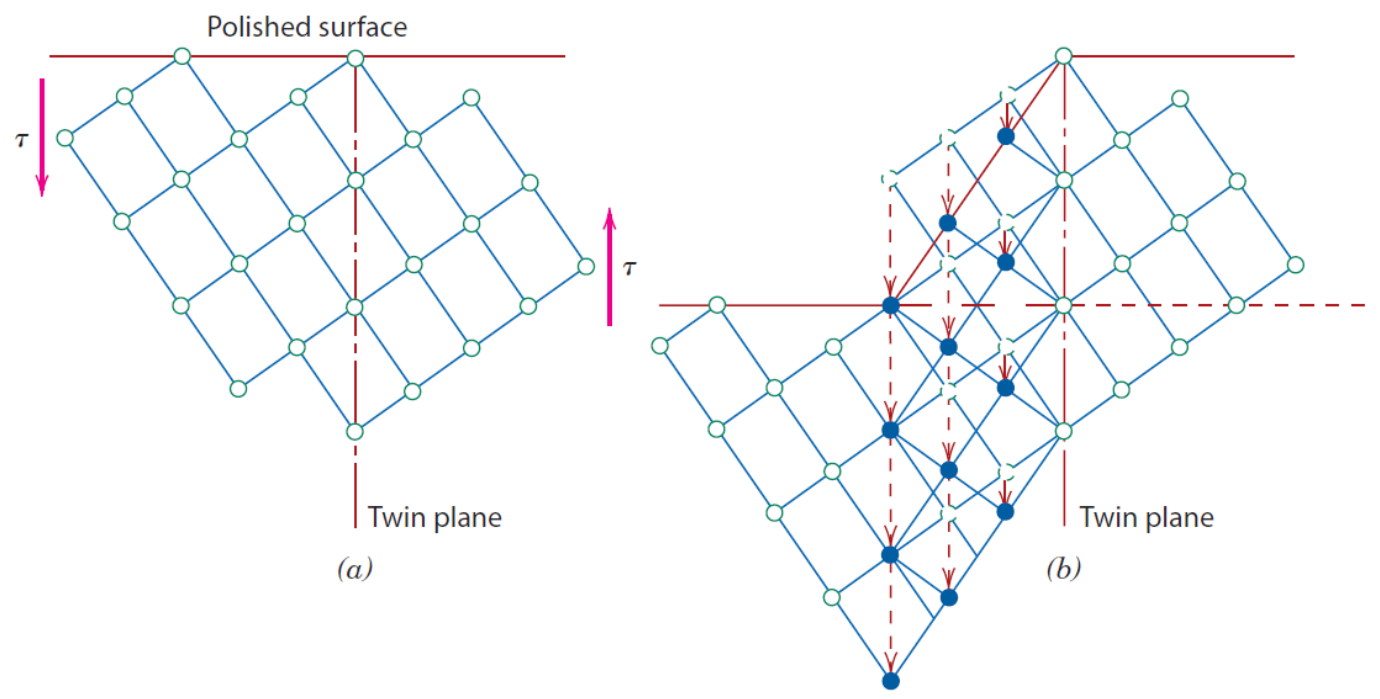

Figure 2.3: (a) Schematic diagram showing how twinning results from an applied shear stress $\tau$, (b) open circles represent atoms that did not change position; dashed and solid circles represent original and final atom positions, respectively [55,56]. 
As a result the region to the right of the twinning plane is undistorted. To the left of this plane, the planes of atoms have sheared in such a way as to make the lattice a mirror image across the twin plane. In a simple lattice such as this, each atom in the twinned region moves by a homogeneous shear a distance proportional to its distance from the twin plane [56]. In Fig. 2.3(b), open circles represent atoms that did not move, and dashed and solid circles represent original and final positions, respectively, of atoms within the twinned region $[55,56]$.

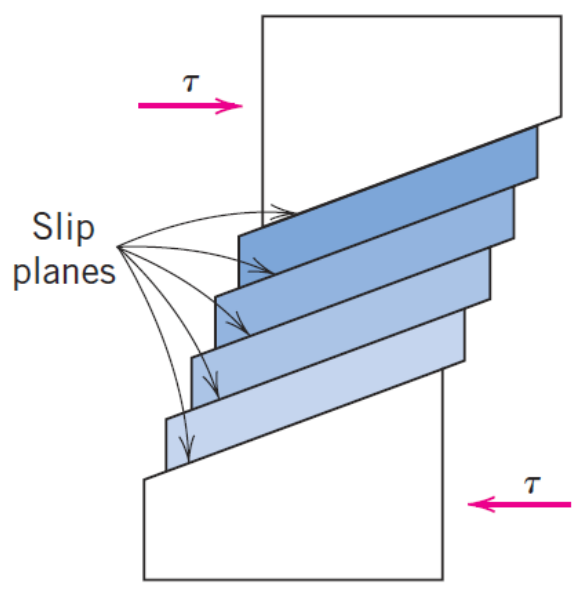

(a)

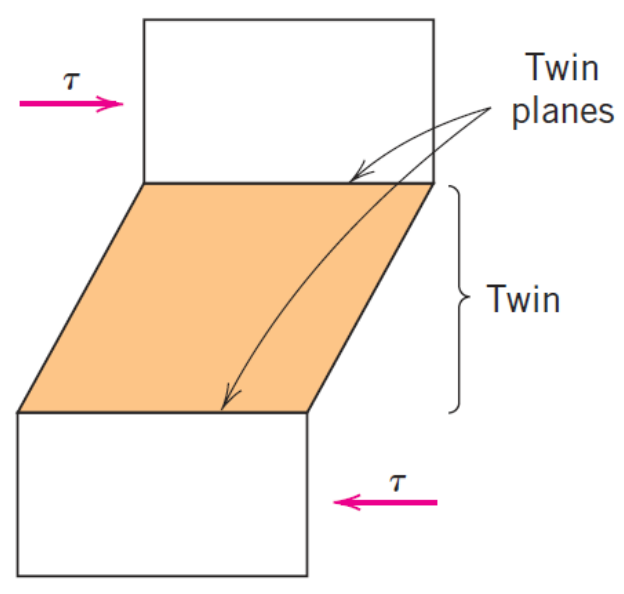

(b)

Figure 2.4: For a single crystal subjected to a shear stress $\tau$, (a) deformation by slip; (b) deformation by twinning [55].

Slip and twinning deformations are compared in Fig. 2.4(a) and (b), respectively. These two processes differ from each other in several respects. First, for slip the crystallographic orientation above and below the slip plane is the same before and after the deformation; for twinning, there will be a reorientation across the twin plane. In addition, slip occurs in distinct atomic spacing multiples, whereas the atomic displacements for twinning are less than interatomic separation [55]. Unlike slip, twinning only allows simple shear in one direction, 
rather than in both the forward and backward directions, thus, it is a polar mechanism. In addition, shear in the one direction is not equivalent to shear in the opposite direction $[57,58]$. As a result, the limited slip systems available and the directionality of the twinning systems are the main reasons for the anisotropy of mechanical properties observed in Mg alloys.

\subsubsection{Twinning Types in Magnesium}

As mentioned above, in the hcp crystal structure the twinning systems are strongly correlated with the $c / a$ ratio of the unit cell [53,59-62]. Generally two types of twins, $\{10 \overline{1} 2\}<\overline{1011}>$ extension and $\{10 \overline{1} 1\}<10 \overline{1} \overline{2}>$ contraction twins, are observed in magnesium alloys [63-72].
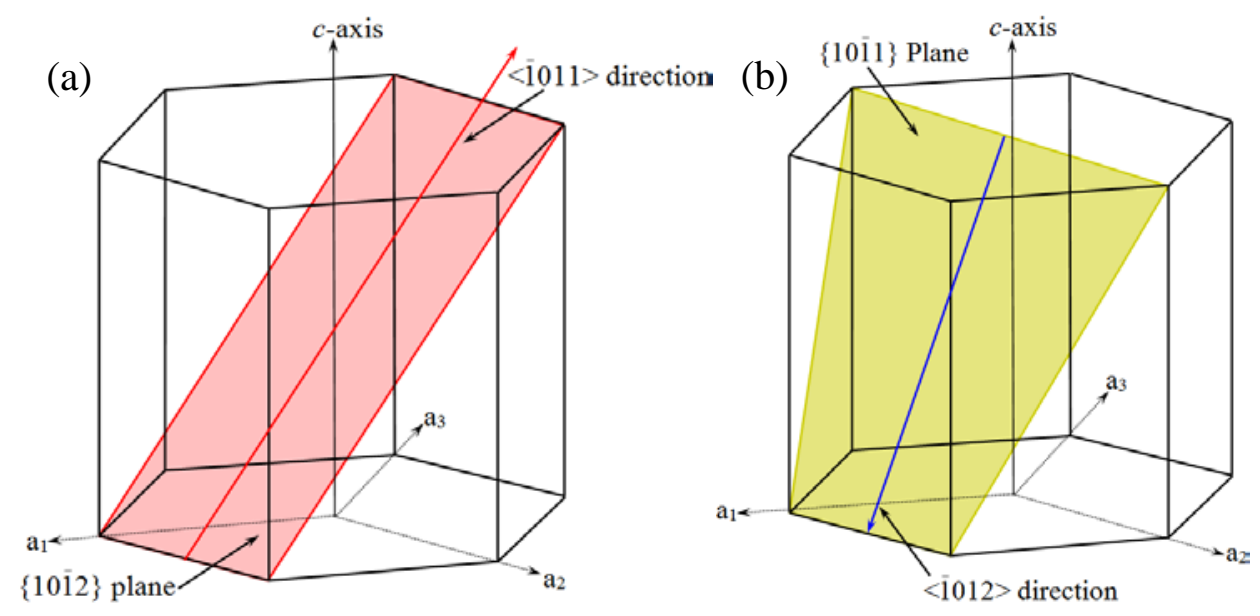

Figure 2.5: (a) Schematic illustration of extension twins $\{10 \overline{1} 2\}<\overline{1011}>$ and, (b) contraction twins $\{10 \overline{1} 1\}<\overline{1012}>$ in magnesium, where the corresponding shear direction are indicated.

The former is the most common and is easily activated in Mg and many other hcp metals $[53,57,61,62]$. The $\{10 \overline{1} 2\}$ extension twins are the only type that allow extension strain parallel 
to the $c$-axis [33]. Thus the $\{10 \overline{1} 2\}$ twins form prevalently when a tensile stress (or a compressive stress) is applied in the direction parallel (or perpendicular) to the $c$-axis. The $\{10 \overline{1} 2\}$ extension twins have the second smallest critical resolved shear stress (CRSS) following basal slip; therefore, the $\{10 \overline{1} 2\}$ twins could be activated in the initial deformation stage and influence yielding behavior. The occurrence of $\{10 \overline{1} 2\}$ twinning causes re-orientation of the basal planes by $86.3^{\circ}$. Therefore, grains that are oriented unfavorably for slip still remain in unfavorable orientations after $\{10 \overline{1} 2\}$ twinning [72].

Contraction twinning, including $\{10 \overline{1} 1\}$ twins and $\{10 \overline{1} 3\}$ twins, on the other hand could accommodate the compression or contraction strain along the $c$-axis [73]. It is activated when there is a contraction strain component parallel to the $c$-axis or when macroscopic extension is applied perpendicular to the $c$-axis. $\{10 \overline{1} 1\}$ and $\{10 \overline{1} 3\}$ contraction twins reorient the basal plane by $56^{\circ}$ and $64^{\circ}$, respectively [74-76]. Like $<\mathbf{c}+\mathbf{a}>$ slip, contraction twinning has the effect of rotating the basal planes towards more favorable orientations for dislocation slip.

In addition to primary twinning, secondary twinning can take place within the reoriented primary twins [64]. Usually reported double twinning are $\{10 \overline{1} 1\}-\{10 \overline{1} 2\}$ and $\{10 \overline{1} 3\}-\{10 \overline{1} 2\}$ twinning. Generally $\{10 \overline{1} 1\}$ or $\{10 \overline{1} 3\}$ contraction twins form first, after which $\{10 \overline{1} 2\}$ extension twins are formed and propagated within the original contraction twins. Both types of double twins lead to a contraction along the c-axis [75]. 


\subsubsection{Twinning Invariants}

Deformation twinning occurs by coordinated dislodgments of atoms along crystallographic directions on crystallographic planes, resulting in homogeneous shearing of the crystal lattice and formation of a new lattice in twin region to the parent lattice [37]. Each twinning mode is identified by the invariant shear plane and shear direction. Twinning elements and a number of other characteristic parameters for $\{10 \overline{1} 2\}$ extension and $\{10 \overline{1} 1\}$ contraction twinning are given in Table 2.2, [61,77].

Table 2.2: Description of the $\{10 \overline{1} 2\}$ and $\{10 \overline{1} 1\}$ twinning invariants [77].

\begin{tabular}{|c|c|c|c|c|c|}
\hline & $K_{1}$ & $K_{2}$ & $\eta_{1}$ & $\eta_{2}$ & $s$ \\
\hline Extension twin & $\{10 \overline{1} 2\}$ & $\{\overline{1012\}}$ & $<10 \overline{1} 1>$ & $<\overline{1} 01 \overline{1}>$ & -0.129 \\
\hline Contraction twin & $\{10 \overline{1} 1\}$ & $\{10 \overline{1} 3\}$ & $<10 \overline{1} 2>$ & $<30 \overline{3} 2>$ & 0.138 \\
\hline
\end{tabular}

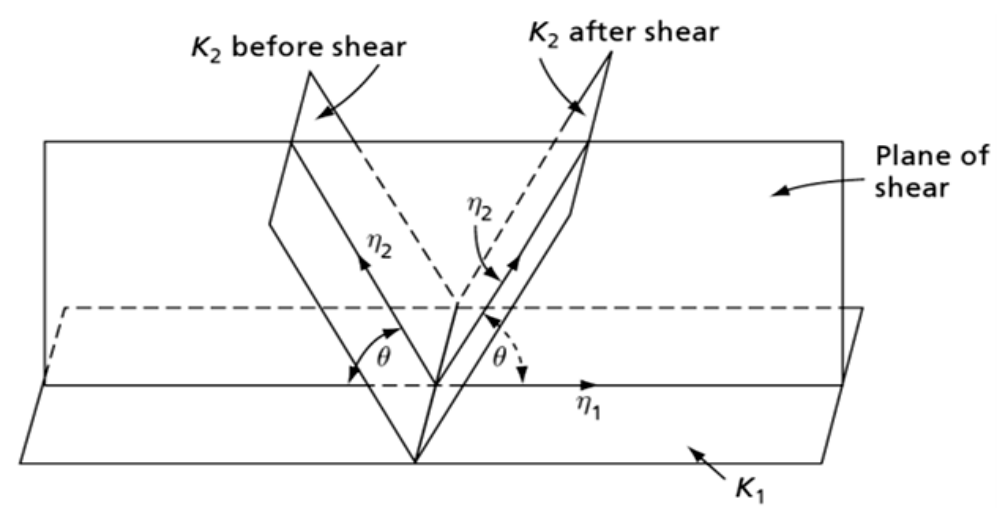

Figure 2.6: Twinning elements $K_{1}, K_{2}, \eta_{1}$ and $\eta_{2}$, shown in relation to the plane of shear [78].

In this table, and with reference to the standard twinning invariants shown in Fig. 2.6, the values $K_{1}$ and $K_{2}$ are the first invariant and second invariant planes, respectively. The terms $\eta_{1}$ and $\eta_{2}$ 
refer to the shear direction and its conjugate, i.e., the vector formed by the intersection of $K_{2}$ and the plane of shear. The symbol $\theta$ refers to the angle through which the basal planes are rotated around the axis $<$ hkil $>$ (which is normal to the plane of shear, Fig. 2.6).

Fig. 2.7 shows a well-known illustrative representation of a twinning shear [37]. The twinning shear occurs in the $K_{1}$ plane along the $\eta_{1}$ direction. The $K_{1}$ plane, the invariant plane, preserves its crystallographic identity in the twin lattice. $K_{2}$ is the conjugate to the twinning plane; it rotates to $K_{2}^{\prime}$ (after shear) in the twin, and it also conserves its type in the twin lattice. The $K_{2}$ plane contains the $\eta_{2}$ direction, defined as the conjugate twinning direction or conjugate shear direction; it rotates during twinning to $\eta_{2}^{\prime}$ in the $K_{2}^{\prime}$ plane in the twin. Another important plane is the plane of the shear, and the projection plane in Fig. 2.7. The plane is extended on $\eta_{1}$ and $\eta_{2}$ or alternatively defined as the plane containing $\eta_{1}$, and the normal to the $K_{1}$ and $K_{2}$ planes. When twinning occurs, the material above the $K_{1}$ plane is sheared by an amount defined by the twinning shear, s. The magnitude of the twinning shear determines the position of the $K_{2}^{\prime}$ plane in the twin lattice. In the hcp lattice the twining shear $s$ depends on the $c / a$ ratio.

The shear magnitude for extension twinning, $s_{\mathrm{e}}$, is expressed by [53]:

$$
s_{e}=\frac{(c / a)^{2}-3}{\sqrt{3}(c / a)}
$$

The shear magnitude for contraction twinning, $s_{\mathrm{c}}$, is expressed by [53]:

$$
s_{C}=\frac{4(c / a)^{2}-9}{4 \sqrt{3}(c / a)} .
$$


(a)
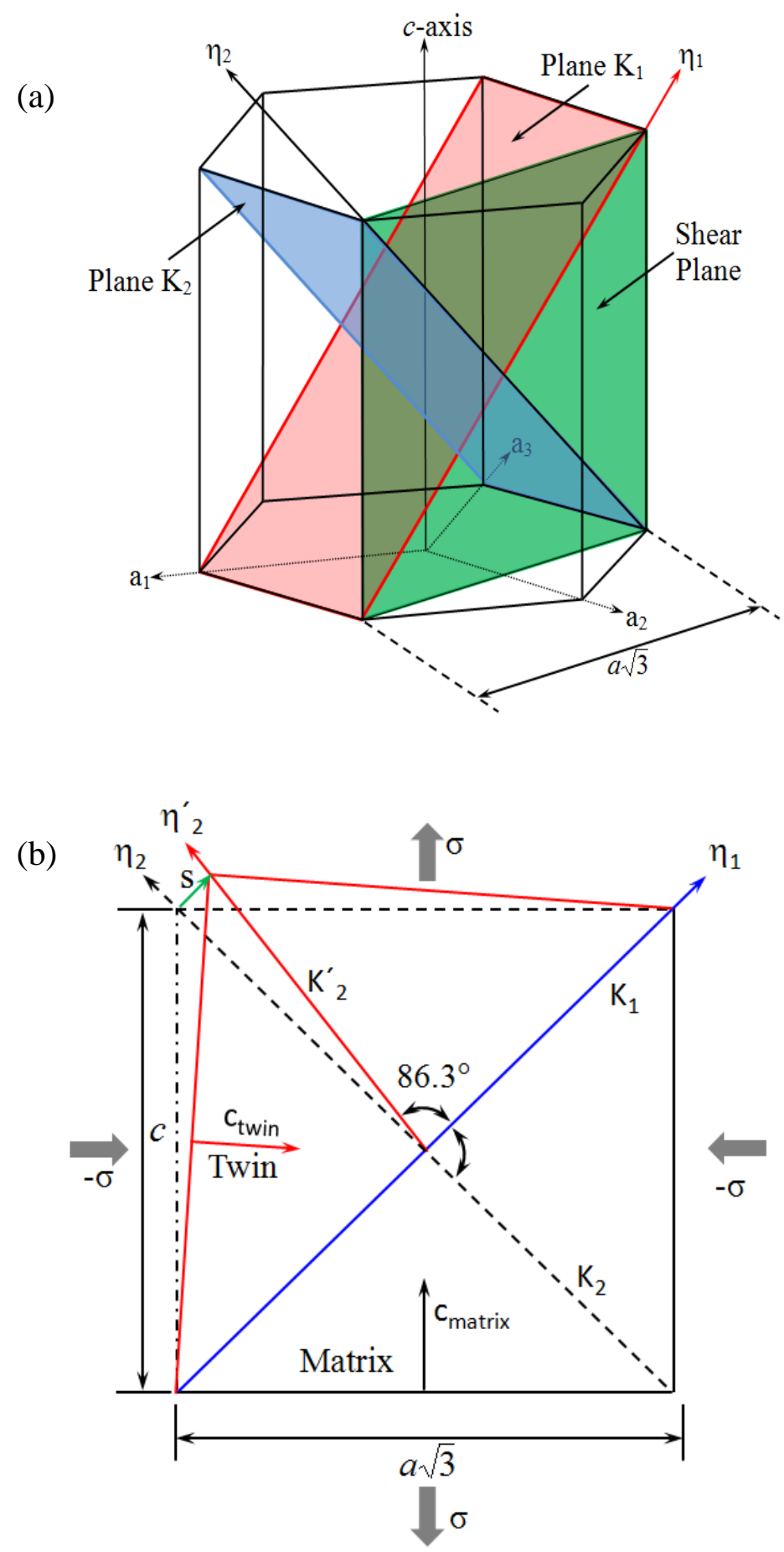

Figure 2.7: (a) Twinning elements of twinning mode $\{10 \overline{1} 2\}<10 \overline{1} 1>$, (b) schematic image of twin mechanism. Extension twin occurs when a compressive load is applied perpendicular to the $c$-axis $[49,79]$. 


\subsubsection{Twin Growth}

Twin nucleation occurs in regions of stress concentration; grain boundaries and other dislocations. Twin growth starts after twin nucleation when the resolved shear stress (RSS) on the twinning system exceeds the critical resolved shear stress (CRSS) for twin growth, which is usually less than the CRSS for twin nucleation [80]. Twin growth is accomplished through gliding of twinning dislocations (TDs) in the matrix on the twin boundary (TB), where the driving force is the stress acting on TDs at the TB interface. Twin growth is related to two phenomenons [80]: matrix reduction and twin propagation, which are respectively activated by the average stresses in the matrix and in the twin. Matrix reduction is accomplished through the migration of the twin boundaries induced by the resolved shear stress in the matrix and thus reduces the current volume of the matrix; twin propagation is accomplished through the migration of the twin boundary towards the matrix by the resolved shear stress in the twin and increases the current volume of the twin.

\subsubsection{De-twinning}

Due to the polar nature of twinning, the extension twin can only be activated by a tensile component of stress along the $c$-axis or by a compressive component of stress normal to the $c$ axis of the hcp unit cell. Because extension twinning results in an $86.3^{\circ}$ reorientation of the basal pole, de-twinning can occur in a twin if the load is reversed [80]. Under cyclic loading and strain path changes, twinning and de-twinning appear alternately [11,34,67,69,81-86]. 
Microscopically, de-twinning can be characterized by the disappearance of existing twins. In other words, twins can vanish or become narrower (twin shrinkage) under reversed loading. Twin shrinkage operates when the resolved shear stress (RSS) corresponding to the de-twinning is greater than the critical resolved shear stress (CRSS) for twin shrinkage. Twin shrinkage is the reverse operation of twin growth. Therefore, a complete texture reversal is expected, once the de-twinning capability is exhausted. The crystallographic deformation process of de-twinning is similar to twinning, except that nucleation is not required, or occurs more readily. Thus, the stress required for de-twinning is less than that for twinning nucleation, but greater than that for twinning growth [49]. Therefore, de-twinning can also result in an inflected and concave-up strain-hardening flow curve [38].

\subsubsection{Re-twinning}

Re-twinning (RT) represents the activation of a twin within a twin. The twin variant could be the same as or different from the pre-existing twin variant. If the variant is the same as the preexisting twin, it corresponds to de-twinning through the nucleation of a twin [86]. If the variant is different from the pre-existing twin, it corresponds to secondary twinning through the nucleation of a new twin variant in the twin. Because re-twinning corresponds to the introduction of a new twin, the CRSS for re-twinning could be the same as the CRSS for twin nucleation. The resolved shear stress corresponding to this operation is calculated using the stress state inside the twin. 


\subsection{Factors Influencing Deformation Mechanisms}

\subsubsection{Critical Resolved Shear Stress}

To activate a slip or twinning system within the crystal, the shear stress on the slip or twin plane in the slip or twin direction must reach a required level called the critical resolved shear stress (CRSS). It constitutes a material property that may be influenced by deformation temperature, impurities and alloying elements, as well as by the mechanical and thermal history (the rate of deformation and of recrystallization). Barnett [87] summarized the temperature dependence of critical resolved shear stress (CRSS) for the main deformation modes in magnesium, as shown in Fig. 2.8. The CRSS for the basal slip in pure magnesium is very low, approximately $0.6-0.7$ $\mathrm{MPa}$, which is also nearly independent of temperature. In contrast, the critical resolved shear stress for the non-basal slip is over $40 \mathrm{MPa}$ at low temperature; this is two orders of magnitude higher than that for the basal slip, and drastically decreases to 2-3 MPa with increasing temperature [88]. In the single crystal Mg, basal slip is the dominant deformation mechanism at room temperature. However, when it comes to polycrystalline Mg, the situation is different since the grains are constrained by the surrounding grain boundaries. If only the basal slip is activated, it can only offer two independent slip systems, which is less than the required five. This will cause strain incompatibility at the grain boundaries. When the grain boundaries are strong enough, stress concentration occurs to maintain strain compatibility at the grain boundaries [39]. This stress concentration would lead to the activation of non-basal slip, as well as twinning, in Mg. 


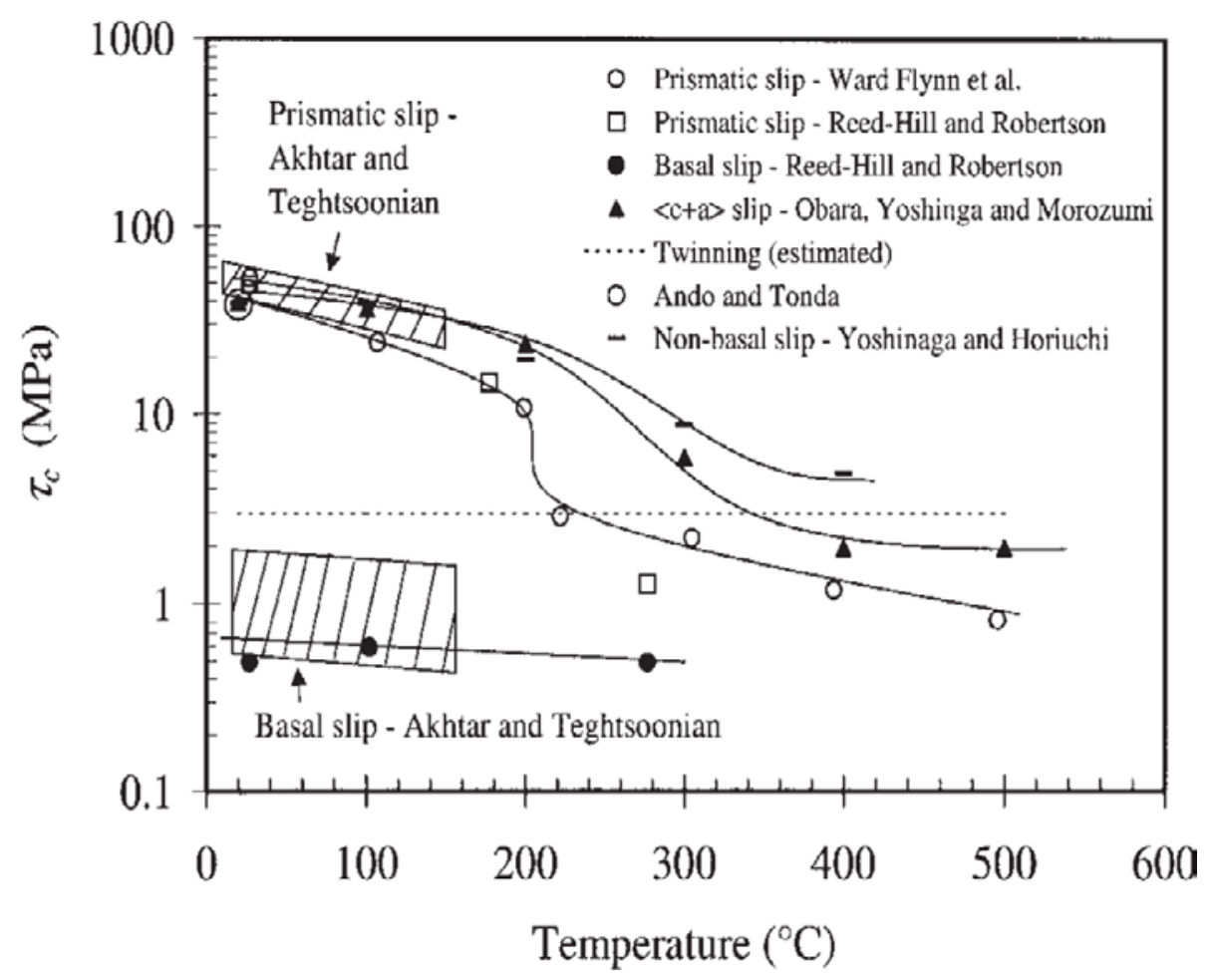

Figure 2.8: Influence of deformation temperature on the critical resolved shear stress [87].

The reason for the easy formation of the $\{10 \overline{1} 2\}$ twins can be attributed to their small CRSS, as indicated by the dotted line in Fig. 2.8. Various parameters related to the formation of $\{10 \overline{1} 2\}$ and $\{10 \overline{1} 1\}$ twins and the most conspicuous difference is found in the CRSS values. The $\{10 \overline{2}\}$ extension twinning requires only 2 to $3 \mathrm{MPa}$, [74] while $\{10 \overline{1} 1\}$ contraction twinning requires 114 MPa [39].

\subsubsection{Schmid Factor and Schmid Law}

The Schmid factor arises from a purely geometrical relationship between the corresponding deformation mode and the direction of the applied force $[55,89]$. The relationship between the 
resolved shear stress operating on the slip/twinning plane along the slip/twinning direction, the direction of the external applied force, and the specimen dimension is given by the following equation and also is given in Fig. 2.9:

$$
\tau_{C}=\frac{F}{A} \cos \varphi \cos \lambda,
$$

where:

- $\tau_{c}$ : resolved shear stress (RSS) on the slip/twinning plane in the shear direction;

- F: externally applied force in the direction of the rod axis;

- A: cross-section area of the rod sample;

- $\varphi$ : angle between the slip/twinning direction and net force of the external reference system.

- $\lambda$ : angle between the normal to the slip/twinning plane and the direction of force of the external reference system. The Schmid factor is the product of $\cos \varphi$ and $\cos \lambda$.

In hcp metals, the resolved shear stresses of the various deformation modes are strongly dependent on the direction of the external applied force, especially with respect to the $c$-axis. When the applied RSS reaches the critical resolved shear stress (CRSS), slip (or twinning) occurs; this is usually called the Schmid law. 


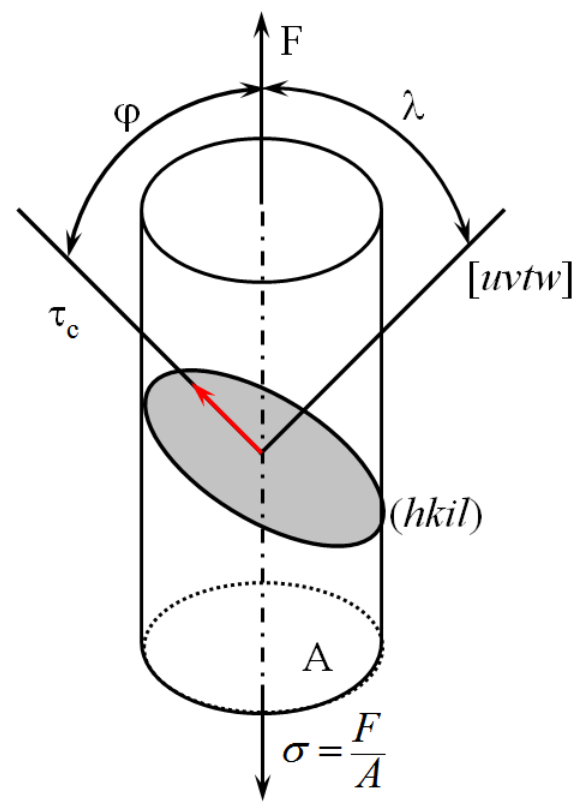

Figure 2.9: Relationship between the resolved shear stress acting on the deformation system and the force applied externally on the single-crystal rod [55].

\subsubsection{Texture (Preferred Orientation)}

Most natural or artificial solid materials are polycrystalline, consisting of many crystallites (also called grains) of various size, shape, and orientation. Each grain is a single crystal with anisotropic properties due to its periodic atomic arrangement in three-dimensional space. The properties of polycrystalline materials are determined by the combination of the single crystal's properties, the boundary between grains, and the orientation distribution of the grains within the material. In some cases, the orientations of the grains in a material have a random distribution, which produces isotropic properties. In most cases, grains have an anisotropic orientation distribution and properties. This anisotropic orientation distribution is referred to as a preferred orientation or a texture [90]. 


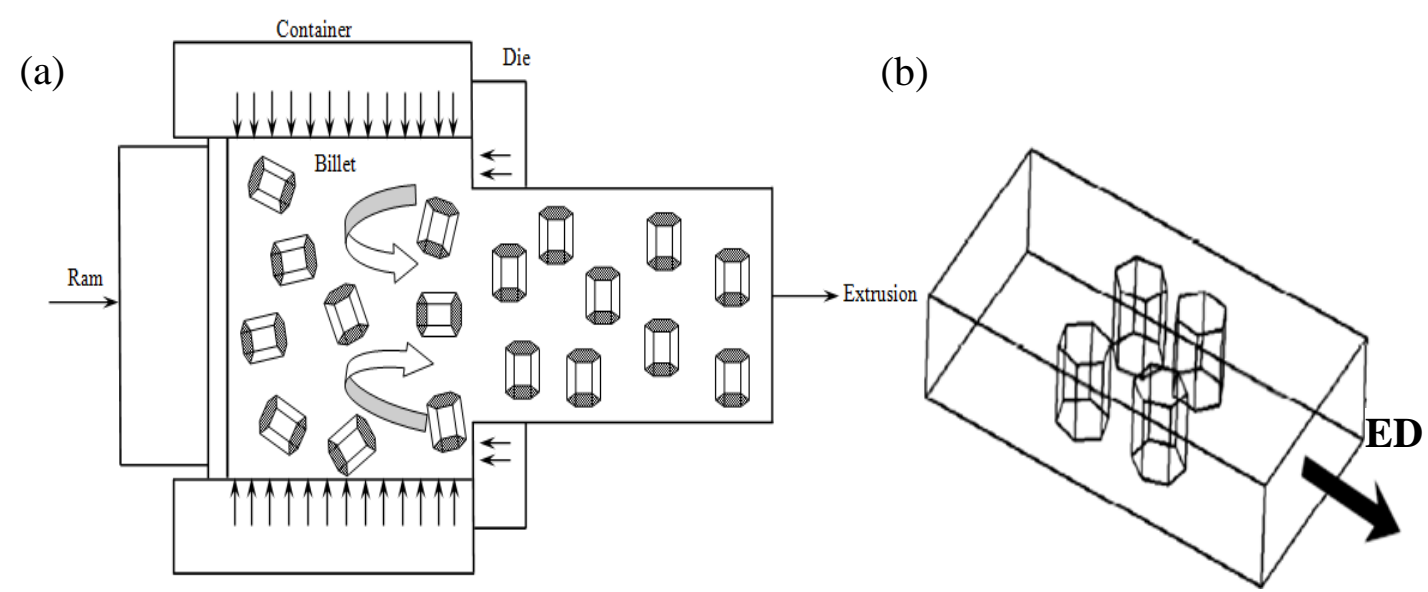

Figure 2.10: Schematic diagram showing the (a) extrusion process, and (b) the formation of basal texture in extruded magnesium [93].

When a metal has undergone a severe amount of deformation during manufacturing processes [38,91-94] such as rolling, extrusion, forging, wire drawing etc., it will develop a preferred orientation, or texture, in which certain crystallographic planes tend to orient themselves in a preferred manner with respect to the direction of maximum strain [56]. A schematic diagram in Fig. 2.10 shows the extrusion process (Fig. 2.10(a)) and the formation of basal texture (Fig. 2.10(b)) during the extrusion.

The preferred orientation resulting from plastic deformation is strongly dependent on the slip and twinning systems available for deformation, but it is not generally affected by such processing variables as die angle, roll diameter, roll speed, and reduction per pass. The most important mechanical variables are the geometry of the flow and the amount of deformation. Thus the same deformation texture is produced whether a rod is made by rolling or drawing. 


\subsection{Effect of Twinning on Flow Stress}

With regard to the contribution of twinning to the deformation behavior, two facts are important: (a) the reorientation induced by twinning is generally more prominent than that produced by slip; (b) twinning in Mg is polarized, namely, extension or contraction along the $c$-axis activates twinning in two different senses and is of two different types [72]. Previous research has shown that the flow curves generated when tension is applied along the $c$-axes of most of the grains differ considerably from those generated when contraction is applied along the relevant $c$-axes [33, 74-76]. Twinning has been demonstrated to occur during the deformation of $\mathrm{Mg}$ and other metals [59-62]. On the other hand, the twin boundaries that have formed can act as barriers to dislocation motion, as do grain boundaries, leading to an increase in the work hardening rate. In addition, they transform glissile dislocations into sessile dislocations within the twin interiors and hence contribute to strengthening via the Basinski mechanism (i.e. the trapping of sessile dislocations inside twins) [68]. On the other hand, they accommodate strain along the $c$-axis, which can decrease the work hardening rate. Furthermore, the lattice rotation introduced by twinning can enhance or reduce the work hardening rate, depending on the type of twin formed.

\subsection{Effect of Twinning on Ductility}

Many researchers have studied the favorable effect of twinning on the ductility of hcp metals. Indeed, during $\{10 \overline{1} 2\}$ extension twinning in $\mathrm{Mg}$, the stress remains relatively stable; after twinning, the orientation of the twinned crystal is similar to that for the $c$-axis compression sample but the failure strain has been increased [72]. $\{10 \overline{1} 2\}$ extension twinning in $\mathrm{Mg}$ also 
enhances strain hardening and appears to increase the uniform elongation. Basinski et al [68] has also emphasized the importance of the hardening effect of twinning. In these studies, the role of twin boundaries as barriers to slip was presented, together with that of hardening from the dislocation transformations taking place within twins. Nevertheless, the role of crystallographic reorientation was not mentioned. However, the latter effect appears to be more important in $\mathrm{Mg}$ materials than in $\mathrm{Ti}$ and $\mathrm{Zr}$ alloys [40]. $\{10 \overline{1} 1\}$ twinning and $\{10 \overline{1} 1\}-\{10 \overline{1} 2\}$ twinning reorient the basal planes by $56^{\circ}$ and $38^{\circ}$, respectively, as discussed above. This means that basal planes originally unfavorably oriented for slip can be reoriented to more favorable orientations, which in turn leads to softening. On the other hand, the high CRSS related to contraction and double twinning can also introduce high local stress concentrations, leading to microcracks or voids [73]. Barnett has suggested that a strong interaction may exist between void formation next to twins and twin-induced flow softening. Either of these can induce localization and premature failure [73]. The amount of strain that twinning can accommodate is directly proportional to the twinning shear and the volume fraction of crystal that has twinned [58]. Thus the amount of strain that may be accommodated by twinning is limited, even under ideal circumstances. In addition, although twinning mechanisms offer more options for plastic accommodation, they generally require an accommodation mechanism of their own in order to avoid large internal stresses that can lead to failure [73].

\subsection{Recent Research on Magnesium}

The mechanical behavior is controlled by the relative strengths and hardening responses of a variety of dislocation slip and twinning modes. At room temperature, the initial texture, straining 
direction, and straining amount play a significant role in determining the activity of the possible deformation modes in polycrystalline materials. The plastic behavior of wrought magnesium alloys is characterized by plastic yielding and hardening asymmetry between the longitudinal and normal direction in both the extruded and rolled conditions. These unusual characteristics have been attributed to the variety and asymmetrical distribution of crystallographic deformation modes and, in particular, the deformation twinning on the $\{10 \overline{1} 2\}$ planes along the $<10 \overline{1} 1>$ directions and limited dominant slip systems. Furthermore, the unique reorientation of the extension twinning between the parent and twinned grains facilitates de-twinning during unloading and/or reversed loading. Therefore, several features are very distinctive in magnesium alloys where twinning makes a substantial contribution to plastic deformation, including a very rapid texture development associated with twin reorientation; an unusual hardening response, and significant texture weakening due to multiple loading directions.

\subsubsection{Twinning and Hardening Behavior}

In wrought magnesium alloys, during forming or manufacturing processes (extrusion, rolling, forging, etc.) almost all grains are oriented with their c-axes of hexagonal close-packed (hcp) unit cells perpendicular to the process direction and forming a strong basal texture. This initial texture combined with the polar nature of the twinning leads to the easy activation of extension twinning when a compressive (or tensile) load acts perpendicular (or parallel) to the $c$-axis [95]. This is the reason for the well known yield asymmetry of wrought magnesium alloys [96], where the yield strength in one direction is controlled by the stress required to activate twinning, while the yield strength in the other direction is controlled by the harder non-basal slip 
mechanisms [41,97]. Previous research has shown that the stress-strain curve characteristic of twinning activation has an unusual skewed or concave-up shape: an abrupt transition from the elastic to plastic deformation, a low yield stress and low hardening rate followed by a higher hardening rate. In contrast, the stress-strain curve characteristic of dislocation-dominated deformation has a concave-down shape: a high yield stress with a smooth transition from the elastic to plastic deformation [24,35-37].

Gharghouri et al. [67] studied the mechanical properties in an extruded Mg-7.7 at.\% Al alloy by means of in-situ neutron diffraction. The occurrence of twinning was easily detected by neutron diffraction, considering that it would lead to an abrupt reorientation of the crystal lattice, reflected by a change in the peak density. The test results were consistent with the activation of $\{10 \overline{1} 2\}$ twinning. The $\{10 \overline{1} 2\}$ twins in an extruded Mg-3Al-Zn alloys under compression tests led to distinctive flow curves characterized by high levels of work hardening and a "concave" shape [63]. The hardening effect was attributed to the effects of twins on slip, rather than to the stress required to activate it. With decreasing grain size and increasing temperature, the deformation mechanism transferred from twinning to dislocation slip-dominant flow.

\subsubsection{Texture Effect on Deformation Mechanisms}

Due to the formation of texture and the different CRSS values of different deformation mechanisms, the mechanical properties of the materials depend not only on the stress direction but also on the stress sign (tension or compression) [38]. Consequently, qualitative explanations for the observed mechanical anisotropy of textured wrought magnesium alloys could be given. 
Yi et al. [22] described the anisotropic mechanical behavior of extruded AZ31 magnesium alloy during both uniaxial tension and compression tests, in relation to the crystallographic texture, by means of in-situ texture measurement and viscoplastic self-consistent simulation. The activity of the basal $<\mathbf{a}>$ slip and extension twinning exerts a significant effect on the mechanical anisotropy during tension while the importance of the $<\mathbf{c}+\mathbf{a}>$ slip increases during compression. Choi et al. [50] performed uniaxial compression tests using cylindrical specimens of a hot-rolled AZ31 Mg alloy in both the rolling and normal directions, and determined that texture evolution, work hardening and macroscopic anisotropy are strongly dependent on the loading direction. An analysis of texture evolution using the viscoplastic self-consistent polycrystals model revealed that the extension twins largely accommodate the deformation at a low strain level for compression tests along the rolling direction. However, as the true strain increases, the activation of the pyramidal $<\mathbf{c}+\mathbf{a}>$ slip accommodates the deformation. On the other hand, the pyramidal $<\mathbf{c}+\mathbf{a}>$ slip as a primary mode and the basal $<\mathbf{a}>$ slip as a secondary mode accommodate the deformation for compression along the normal direction.

\subsubsection{Effect of Pre-compression and Loading Direction on Twinning and De-twinning}

The effect of $\{10 \overline{1} 2\}$ extension twinning generated by pre-straining on the subsequent deformation behavior with a change of strain path during reloading has been studied by several authors [11,20,21,98-100]. Kleiner and Uggowitzer [38] investigated the compressive plastic pre-deformation along the extrusion direction on an AZ61 Mg alloy up to pre-strain values of 1, 2 and $3 \%$. Then those samples were subjected to tensile tests following the pre-compressive deformation. It was found that $\{10 \overline{1} 2\}<10 \overline{1} 1>$ twinning leads to a reorientation of $86.3^{\circ}$ of the 
crystal lattice, which caused all basal planes in the twinned regions to become nearly perpendicular to the extrusion direction. As a result, these twinned regions were capable of detwinning during reloading in tension. The mechanical responses have shown that all the strain caused by twinning during compressive plastic pre-deformation was recovered by de-twinning during tensile reloading.

Twinning and de-twinning activities were also detected in the cyclic deformation process of extruded magnesium alloy AZ31 with a strong basal texture under plastic strain amplitude and stress amplitude control at room temperature [13-16]. During the cyclic loading, a large number of twins formed under compressive loading from an in-situ EBSD measurement of the texture. With decreasing compression load, some twins became narrower and shorter while the majority of twins remained almost unchanged at that stage. With the transition from a compressive load to a tensile load, a significant amount of twins became narrower and shorter or even disappeared [101]. Wu et al. [82] confirmed the occurrence of alternative twinning and de-twinning behavior in a wrought ZK60A Mg alloy with the cyclic loading using in-situ neutron scattering; that is, most twins formed during compression were removed via de-twinning when the load was reversed.

Park et al. [11] investigated the twinning behavior of a rolled Mg alloy subjected to consecutive in-plane compressions along two orthogonal directions using in-situ electron backscatter diffraction (EBSD) technique. In their experiment, specimens were compressed to $6 \%$ along the rolling direction and unloaded. Then they were further compressed to 3 and $6 \%$ along the transverse direction. The result exhibited multiple twinning modes: $\{10 \overline{1} 2\}$ twinning in residual 
parent grains, $\{10 \overline{1} 2\}-\{10 \overline{1} 2\}$ double twinning in twin bands, and $\{10 \overline{1} 2\}$ de-twinning in twin bands. This pre-compression effect on plastic deformation behavior of AZ31 Mg alloy has also been observed by Xin et al. [20] who concluded that during re-compression, $\{10 \overline{1} 2\}$ extension twinning can occur within the pre-existing $\{10 \overline{1} 2\}$ twins and constituted a new type of 'double extension twinning'.

The reverse motion of $\{10 \overline{1} 2\}$ twin boundaries, i.e., de-twinning, was also observed by Molnár et al. [102], during successive compression of a hot-rolled AZ31 alloy at 3.5\% strain along the rolling direction (RD) and then along the normal direction (ND). During the initial compression along the $\mathrm{RD},\{10 \overline{1} 2\}$ twinning was the main deformation mode, whereas a twin-free microstructure was observed after subsequent compression along the ND which restored the initial shape of the sample. Xin et al. [103] examined the plastic deformation behavior of a hotrolled AZ31 alloy under 6 passes of plain compression along the transverse direction (TD) and ND alternatively, and reported that at $10 \%$ compression along the TD most grains were nearly twinned and extension twinning took place during subsequent compression along the ND without any de-twinning. However, Proust et al. [83] studied the effect of strain path change on twinning and de-twinning where an AZ31-H24 alloy was pre-strained in-plane and then reloaded along the through-thickness ND direction, and observed that the twinned region of the grain that had been created during the loading phase of the deformation began to shrink until it disappeared totally and the grain was then constituted entirely of matrix. Similar results of detwinning were observed by Brown et al. [98] in a rolled beryllium which was initially deformed in-plane and then in the through-thickness ND direction. 


\subsubsection{Annealing and Its Influence on Twinning and Texture}

One of the seminal pieces of work on recrystallization in Mg alloys was by Ion et al. [36] on Mg-0.8\%Al during deformation at elevated temperatures (above 423K). Since then, there has been considerable amount of work on dynamic recrystallization (DRX) in Mg alloys [104-107]; however far less attention has been paid to static recrystallization (SRX) of Mg alloys deformed mainly at room temperature [108-110].

Recrystallization could potentially be a powerful tool in improving the formability of magnesium alloys by altering their texture for at least two important reasons. First, as recrystallization nuclei originate in the deformed structure [111], deformation twins offer the possibility to form new recrystallized orientations that are not easily achieved by slip alone. In particular, contraction and double twins offer the potential for forming many new orientations that would be ideal candidates to influence the recrystallization texture in favor of weakening the strong basal or $c$-axis texture. Second, it has been observed that magnesium alloys exhibit extremely high strain hardening rates for orientations where the $c$-axis is perpendicular during compression [75,112]. As recrystallization requires a minimum amount of stored energy [111], the high strain hardening rates should provide the necessary driving force to initiate recrystallization at low strains, and result in a larger processing window to explore the effects of recrystallization.

Levinson et al. [113] reported the microstructure and texture evolution during isothermal static annealing of an AZ31 magnesium alloy in the cast and extruded states to evaluate the contribution of extension and contraction twins to the recrystallized microstructure after room 
temperature compression to two strains of $5 \%$ and $15 \%$ and then subjected to annealing at temperatures of $175^{\circ} \mathrm{C}$ and $275^{\circ} \mathrm{C}$. Their results showed that contraction and double twins were potent sites for recrystallized nuclei and produced a distribution of new orientations, while no significant influence on the final texture was observed. Yang et al. [114] also studied the effect of prior strain on static recrystallization of a hot-deformed AZ31 magnesium alloy during isothermal annealing at temperatures of $220^{\circ} \mathrm{C}, 230^{\circ} \mathrm{C}$ and $240^{\circ} \mathrm{C}$. It was observed that ultrafine grains were evolved by grain fragmentation due to continuous dynamic recrystallization during hot deformation, and grain coarsening occurred during the subsequent annealing together with a significant change in the deformation texture.

Li et al. [108] has determined the macrotextures and microstructures of a hot extruded AZ31 magnesium plate during static recrystallization using the EBSD technique. At favorable orientations, both extension and contraction twins were formed by rolling and a comparison was made between their recrystallization characteristics. The results again showed that contraction twins are more effective as nucleation sites than extension twins and the orientation characteristics in local regions during nucleation are similar to those of sub-grains within contraction twins or shear bands. During annealing the growth of grains are easier in wider twins, and resulted weakening of the deformation texture.

\subsection{Summary}

Twinning in hcp materials plays two important roles: it has a significant influence on the texture evolution and it strongly affects the mechanical behavior. As briefly reviewed above, the 
occurrence of deformation twinning in magnesium alloys has been widely reported in the literature. However, its precise influence on the hardening behavior is still not well understood and some controversy still remains. Rohatgi et al. [115] explained that the effect on strain hardening rate is due to the grain refinement that results from twinning. By contrast, Barnett [37] pointed out that twin formation in samples aligned for $c$-axis extension, which re-oriented the crystal lattice by almost $86.3^{\circ}$, had a more significant effect on strain hardening than the grain refinement produced by twinning. Knezevic et al. [112] also mentioned that extension twins are not very effective in refining the grain size, and therefore have no significant effect on strain hardening. Their contribution to hardening is through texture hardening, because extension twins rotate grains into hard orientations with the $c$-axis more or less parallel to the loading axis. Jiang et al. [116] pointed out that twin-induced barrier to dislocation motion play a more important role in increasing the strain hardening rate than twin-induced grain reorientation.

Also, the effect of sample orientation on the mechanical behavior and texture evolution under monotonic and cyclic loading has not yet been well understood. Although the sample orientation has a strong influence on strain hardening in magnesium and its alloys, the mechanisms causing this effect are still not clear.

Although twinning and de-twinning in hcp materials such as magnesium and zirconium have been reported in the literature, and they have a significant influence on the strain hardening, the mechanisms causing twinning and de-twinning are still not fully understood. The microstructure evolution that influences hardening and texture behavior upon re-loading (in both longitudinal and transverse directions) depend on pre-strain amounts. However, it is unclear how twinning 
and de-twinning occur with a change of strain path, if the formation of new twins and detwinning can be co-existent, and how they affect the texture change and hardening behavior. Moreover, no such studies are seen in the open literature on the recrystallization during annealing of AM30 magnesium alloy after pre-compression at room temperature. It is unknown how the strain hardening rate and texture change occurred in the pre-deformed and annealed alloy during re-compression. This dissertation is aimed to deal with these unclear or unknown issues. 


\section{CHAPTER 3}

\section{Materials and Experimental Details}

The microstructural characterization with a focus on texture analysis has been employed to study the mechanical behavior and the role of extension twinning in an AM30 magnesium alloy, as a representative material with typical extension twins. In the following, the description will cover the compression tests as the main testing methods to investigate the loading parameters, loading direction, sample orientation, and heat-treatment on the mechanical behavior and deformation mechanisms of the extruded magnesium alloy. All experimental data was carefully collected with the help of data acquisition instruments connected to the various testing systems.

\subsection{Experimental Materials}

The current research involves an extruded magnesium alloy, AM30, as the experimental material. This alloy was selected as the model material because it is a common wrought magnesium alloy with improved formability (i.e., based on the considerations of practical interest and commercial availability), and it exhibits a strong basal texture, typical for the extruded magnesium alloys. The nominal chemical composition of this magnesium alloy is listed in Table 3.1. 
Table 3.1: Chemical composition (wt \%) of the extruded AM30 magnesium alloy.

\begin{tabular}{|c|c|c|c|c|c|c|c|}
\hline Mg alloy & Al & Mn & Zn & Fe & Ni & Cu & Mg \\
\hline AM30 & 3.4 & 0.33 & 0.16 & 0.0026 & 0.0006 & 0.0008 & Bal. \\
\hline
\end{tabular}

\subsection{Sample Design for Compression Tests}

For the uniaxial compression tests, two types of samples were designed and machined from the AM30 magnesium alloy plate. First type: cylindrical samples with a diameter of $5 \mathrm{~mm}$ and height of $8 \mathrm{~mm}$ based on ASTM E9-09, where the cylinder (or compression) axis is parallel to the ED, as shown in Fig. 3.1(a).

(a)

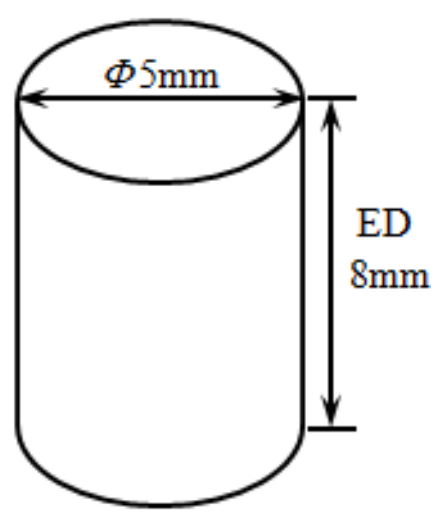

(b)

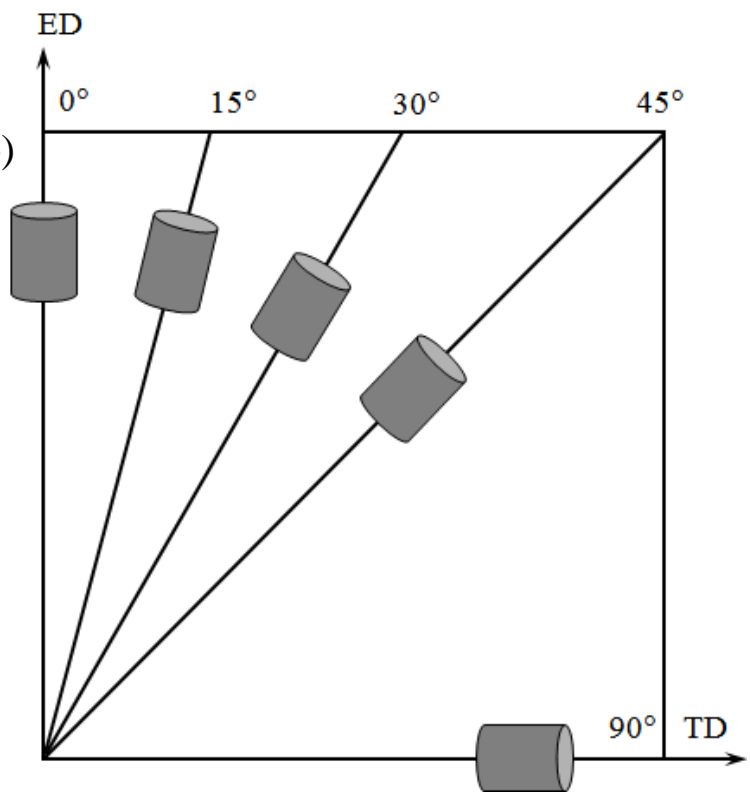

Figure 3.1: Sample dimension for the compression test prepared according to ASTM E9-09 standards for, (a) cylindrical sample, (b) schematic diagram showing the orientation of samples with respect to the extrusion direction (ED). 
To observe the effect of sample orientation during compression, some cylindrical specimens ( $\Phi 5$ $\mathrm{mm} \times 8 \mathrm{~mm}$ ) were machined with the cylinder (compression) axis oriented at $0^{\circ}, 15^{\circ}, 30^{\circ}, 45^{\circ}$ and $90^{\circ}$ to the extrusion direction (ED), as illustrated in Fig. 3.1(b). Second type: Rectangular samples with dimensions of $5 \mathrm{~mm} \times 4 \mathrm{~mm} \times 6 \mathrm{~mm}(\mathrm{ED} \times \mathrm{TD} \times \mathrm{ND})$ were used as the samples permitted loading along the three different strain directions with the same sample geometry. The geometry and dimensions of samples is shown in Fig. 3.2. Such a sample design also allows an easy preparation of flat surface to facilitate twin observations at the end of each deformation step.

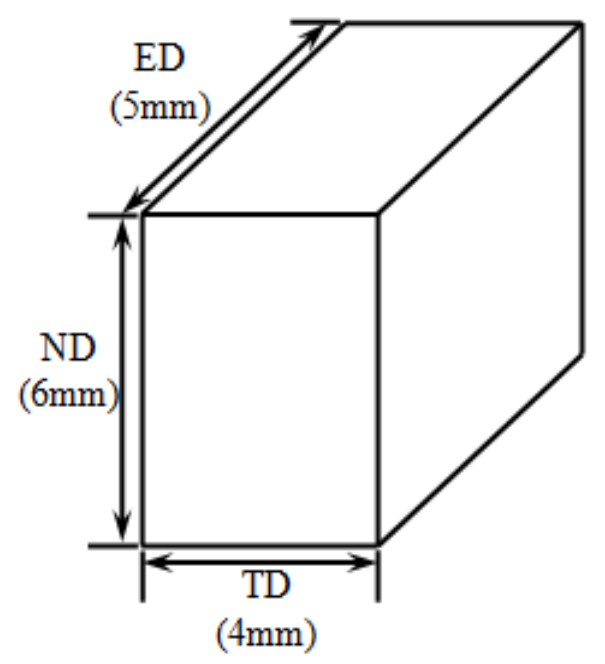

Figure 3.2: Rectangular sample dimension for the compression test.

\subsection{Uniaxial Compression Tests}

All the tests were conducted using a fully computerized Instron machine at Ryerson University. The displacements and load were converted into true strain and true stresses in the standard way 
while neglecting the elastic strains [56]. As there was no extensometer for direct extension measurement from all additional deformation such as load frame, load cell, grips or fixtures, the load displacement curve from the machine by touching the compressive plate to plate was evaluated and considered as machine deformation. It should be noted that in the strain-related evaluation (i.e., strain amounts, stress-strain curves and strain-hardening rates); the machine deformation was eliminated using a calibration curve to arrive at the actual or net strain values of all test samples. To do the compression test, the following experimental parameters were chosen:

1. For the results presented in Chapter 4, to observe the twinning and texture development, compression tests were first performed with cylindrical specimens $(\Phi 5 \mathrm{~mm} \times 8 \mathrm{~mm})$ up to failure at a strain rate of $1.25 \times 10^{-4} \mathrm{~s}^{-1}$ and at room temperature. Then some samples were tested at different strain levels of $1.5,2.4,4.3,8.4$, and $12.9 \%$. After each compression test, the deformed samples were cut along the compression axis and prepared for texture analysis. To detect the twin growth as a function of strain, rectangular samples $(5 \mathrm{~mm} E D \times 4 \mathrm{~mm} \mathrm{TD} \times 6 \mathrm{~mm}$ ND) were compressed along the ED at a compressive true strain of $1.7 \%$, and then a true strain increment of $0.91 \%$ was applied in steps for a total of 9 steps. Twin growth morphology was observed via optical microscopy, after polishing of the $\mathrm{ED} \times \mathrm{ND}(5 \mathrm{~mm} \times 6 \mathrm{~mm})$ sample surface using standard metallographic techniques.

2. For the results presented in Chapter 6, to observe the effect of pre-strain and loading direction on the microstructure and mechanical behavior of AM30 alloy, three types of loading directions were chosen as follows: 
i. Cylindrical samples ( $\Phi 5 \mathrm{~mm} \times 8 \mathrm{~mm}$ ) were pre-strained in compression at different strain levels of $1.5 \%, 2.3 \%, 4.0 \%, 5.8 \%, 7.5 \%$ and $8.2 \%$ (denoted as $1.5 \% \mathrm{ED}, 2.3 \% \mathrm{ED}$, 4.0\%ED, 5.8\%ED, 7.5\%ED and 8.2\%ED, respectively), then re-loaded until failure along the ED, as symbolized by $x \% E D-E D$, where $x$ indicates the pre-strain amount after the exclusion of machine deformation. Some tests were stopped at a strain of $4.0 \%$, to observe the microstructure and texture changes at that strain.

ii. The first set of rectangular samples $(5 \mathrm{~mm} E D \times 6 \mathrm{~mm} \mathrm{TD} \times 4 \mathrm{~mm} \mathrm{ND})$ were pre-strained along the ED at different strain levels of $1.7 \%, 5.2 \%$, and $7.5 \%$ (named as $1.7 \% \mathrm{ED}$, $5.2 \% \mathrm{ED}$, and $7.5 \% \mathrm{ED}$, respectively). The pre-strained rectangular samples were subsequently subjected to re-compression along the TD until failure and symbolized by $x \%$ ED-TD. To observe the microstructural change and texture evolution, some predeformed samples were re-deformed to a strain amount of $4.0 \%$.

iii. A second set of rectangular samples $(5 \mathrm{~mm} \mathrm{ED} \times 4 \mathrm{~mm} \mathrm{TD} \times 6 \mathrm{~mm} \mathrm{ND})$ were pre-strained along the ED at different strain levels of $2.1 \%, 3.7 \%, 5.4 \%$ and $7.9 \%$ (named as 2.1\%ED, 3.7\%ED, 5.4\%ED, and 7.9\%ED, respectively). The pre-strained rectangular samples were subjected to recompression along the ND until failure and symbolized by $x \%$ ED-ND. To study the microstructural and texture evolution, some pre-deformed samples were re-deformed to a strain amount of 3.7\% along the ND. 
3. For the results presented in Chapter 7, to observe the effect of pre-compression and subsequent annealing on microstructure and texture formation of AM30 alloy, cylindrical samples $(\Phi 5 \mathrm{~mm} \times 8 \mathrm{~mm}$ ) were pre-strained along the ED at a strain level of $4.3 \%$ (denoted as 4.3\%ED) with a constant strain rate of $1.25 \times 10^{-4} \mathrm{~s}^{-1}$ at room temperature. Some pre-strained cylindrical samples were annealed at $250^{\circ} \mathrm{C}$ and $350^{\circ} \mathrm{C}$ for $3 \mathrm{~h}$ and other pre-strained samples at $450^{\circ} \mathrm{C}$ for $0.25 \mathrm{~h}, 1 \mathrm{~h}, 3 \mathrm{~h}, 6 \mathrm{~h}, 12 \mathrm{~h}, 24 \mathrm{~h}$ and $60 \mathrm{~h}$. The annealed samples at different temperatures and times $\left(250^{\circ} \mathrm{C}\right.$ and $350^{\circ} \mathrm{C}$, for $3 \mathrm{~h} ; 450^{\circ} \mathrm{C}$ for $3 \mathrm{~h}, 6 \mathrm{~h}$, and $\left.12 \mathrm{~h}\right)$ were subsequently subjected to re-compression along the ED until failure. To observe the microstructural change and texture evolution, some pre-deformed and annealed samples at $450^{\circ} \mathrm{C}$ for $3 \mathrm{~h}$ and $12 \mathrm{~h}$, were selected and re-deformed to a strain amount of $4.3 \%$ along the ED.

\subsection{Heat Treatment-Annealing}

A schematic diagram representing the temperature and time for annealing is shown in Fig. 3.3.

One set of cylindrical samples were annealed at $250^{\circ} \mathrm{C}$ and $350^{\circ} \mathrm{C}$ for $3 \mathrm{~h}$ and the other set of samples at $450^{\circ} \mathrm{C}$ for $0.25 \mathrm{~h}, 1 \mathrm{~h}, 3 \mathrm{~h}, 6 \mathrm{~h}, 12 \mathrm{~h}, 24 \mathrm{~h}$ and $60 \mathrm{~h}$ as shown in Table 3.2. After annealing, all samples were cooled by air.

Table 3.2: Temperature and time selected during annealing.

\begin{tabular}{|c|c|}
\hline Annealing temperature, ${ }^{\circ} \mathrm{C}$ & Holding time, $\mathrm{h}$ \\
\hline 250 & 3 \\
\hline 350 & \multirow{2}{*}{$\begin{array}{c}0.25,1,3,6,12 \\
24,60\end{array}$} \\
\hline \multirow{2}{*}{450} & \\
\hline
\end{tabular}




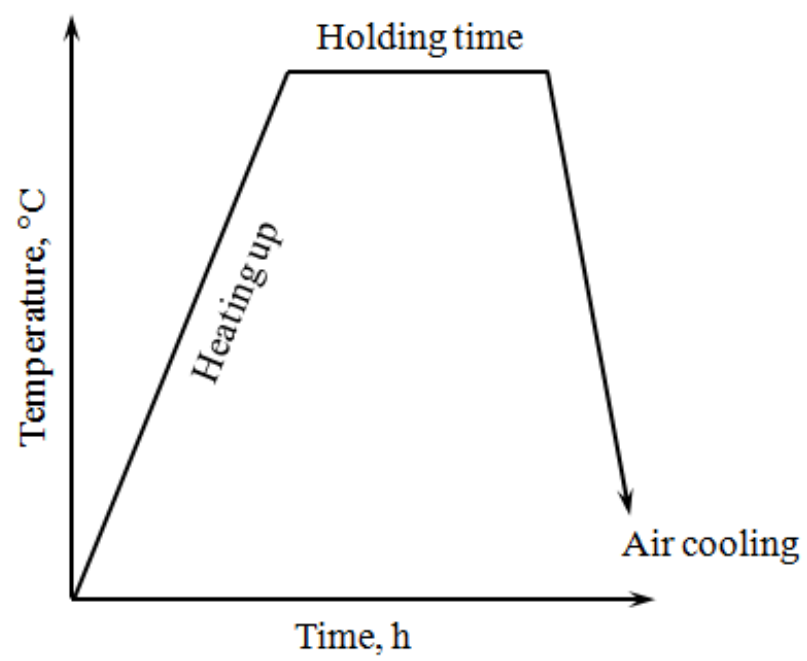

Figure 3.3: Schematic diagram showing the variables, temperature and time during annealing.

\subsection{Metallography}

For the microstructural characterization and texture measurements using the lab XRD, the deformed or re-deformed samples were cut along the compression axis using a slow diamond cutter, cold-mounted, ground using SiC paper up to a grit of \#1200, and polished with 6, 3, and $1 \mu \mathrm{m}$ diamond paste, respectively. After an ultrasonic cleaning, the samples were finally polished with colloidal silica, and etched using an acetic picral solution containing 4.2-g picric acid, 10-ml acetic acid, 10-ml $\mathrm{H}_{2} \mathrm{O}$, and 70-ml ethanol to examine the evolution of deformation twins during compression. The microstructures were examined using Nikon EPIPHOT200, a light optical microscope, equipped with a quantitative image analyzer and CLEMEX software. 


\subsection{Twin Volume Fraction Measurements}

A point counting procedure according to ASTM E562-11 was used to evaluate the volume fraction of twins.

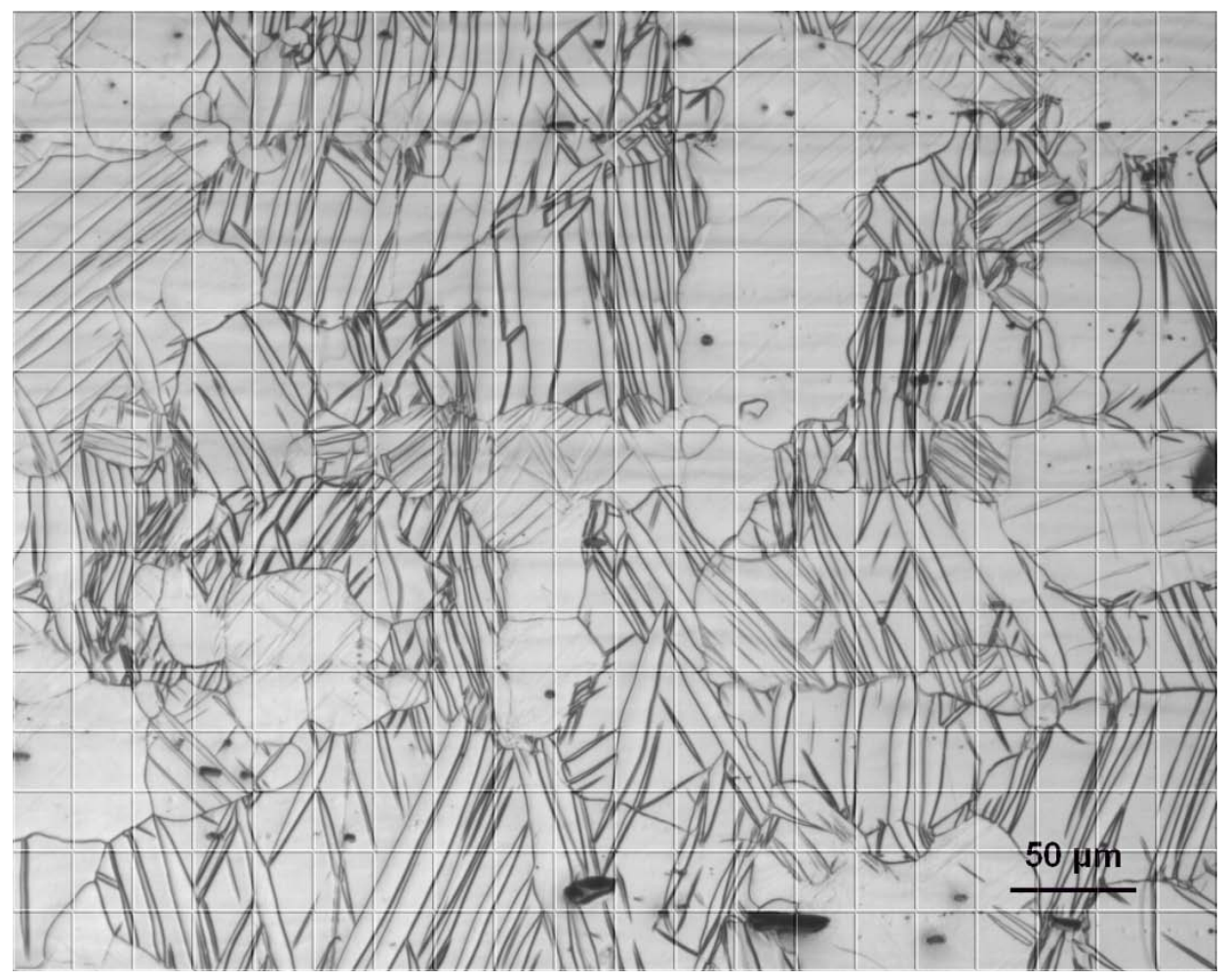

Figure 3.4: An example showing how to evaluate the volume fraction of twins via a point counting technique. In this example the volume fraction is obtained to be $182 / 357=51 \%$.

Five digital micrographs were taken from each sample at a fixed magnification of $200 \times$, and then a grid of 17 points by 21 points (= 357 grid points) was superimposed on the images. The volume fraction was determined by calculating the ratio of the number of points positioned within the twins to the total number of grid points. Fig. 3.4 illustrates an example of this 
technique. This procedure was repeated five times per sample in order to obtain representative values of the fraction of material that had twinned.

\subsection{Quantitative Texture Analysis}

Pole figure is a representation of the orientation distribution of all grains in a sample with respect to the sample surface (rolling or extrusion surface) and sample direction (e.g., the extrusion direction). X-ray diffraction is the most popular method for the texture measurement. Texture characterization by X-ray diffraction involves the measurement of the peak intensity of a particular crystallographic plane at all possible tilt angles with respect to a sample surface and direction.

The schematic diagram in Fig. 3.5 shows the principle: in order to determine the orientation of a given lattice plane, $h k l$, the detector is first set to the proper Bragg angle, $2 \theta$, of the diffraction peak of interest. Then the sample is rotated along two independent axes $\phi\left(\phi=360^{\circ}\right.$; step size $5^{\circ}$, total steps 72$)$ and $\Psi\left(\Psi=75^{\circ}\right.$; step size $5^{\circ}$, total steps 15$)$ in a goniometer until the lattice plane $h k l$ is in the reflection condition (i.e., the normal to the lattice plane or diffraction vector is the bisectrix between incident and diffracted beam, as shown in Fig. 3.5). 


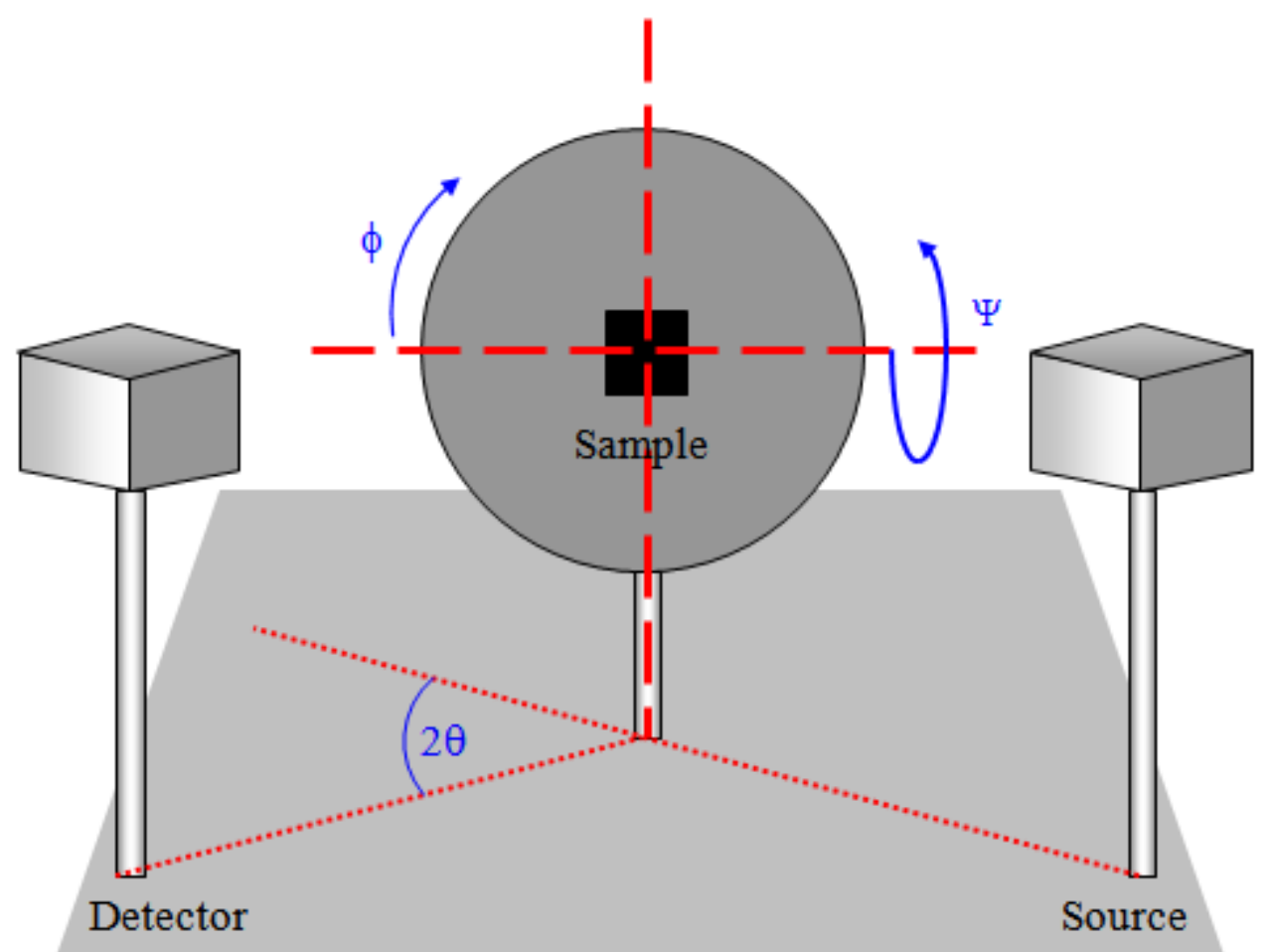

Figure 3.5: Schematic illustration of texture measurements using X'pert MRD system.

Plotting the intensity of each $(h \mathrm{kl})$ plane with respect to the sample coordinates in a stereographic projection gives a qualitative view of the orientation of the crystallites with respect to a sample direction. These stereographic projection plots are called pole figures. As shown in Fig. 3.6(a), the extrusion direction (ED) is aligned to the sample coordinate $S_{2}$, the transverse direction (TD) to $S_{1}$, and the plate normal direction (ND) to $S_{3}$. Let us consider a sphere with unit radius and the origin at $O$. A unit vector representing an arbitrary pole direction (also the unit vector of the diffraction) starts from the origin $O$ and ends at the point $P$ on the sphere. The pole direction is defined by a set of the radial angle $\Psi$ and azimuthal angle $\phi$. The pole density at the point $P$ projects to the point $P^{\prime}$ on the equatorial plane through a straight line from $P$ to the 
point $S$. The pole densities at all directions are mapped onto the equatorial plane by stereographic projection.

(a)

$\mathrm{ND}$

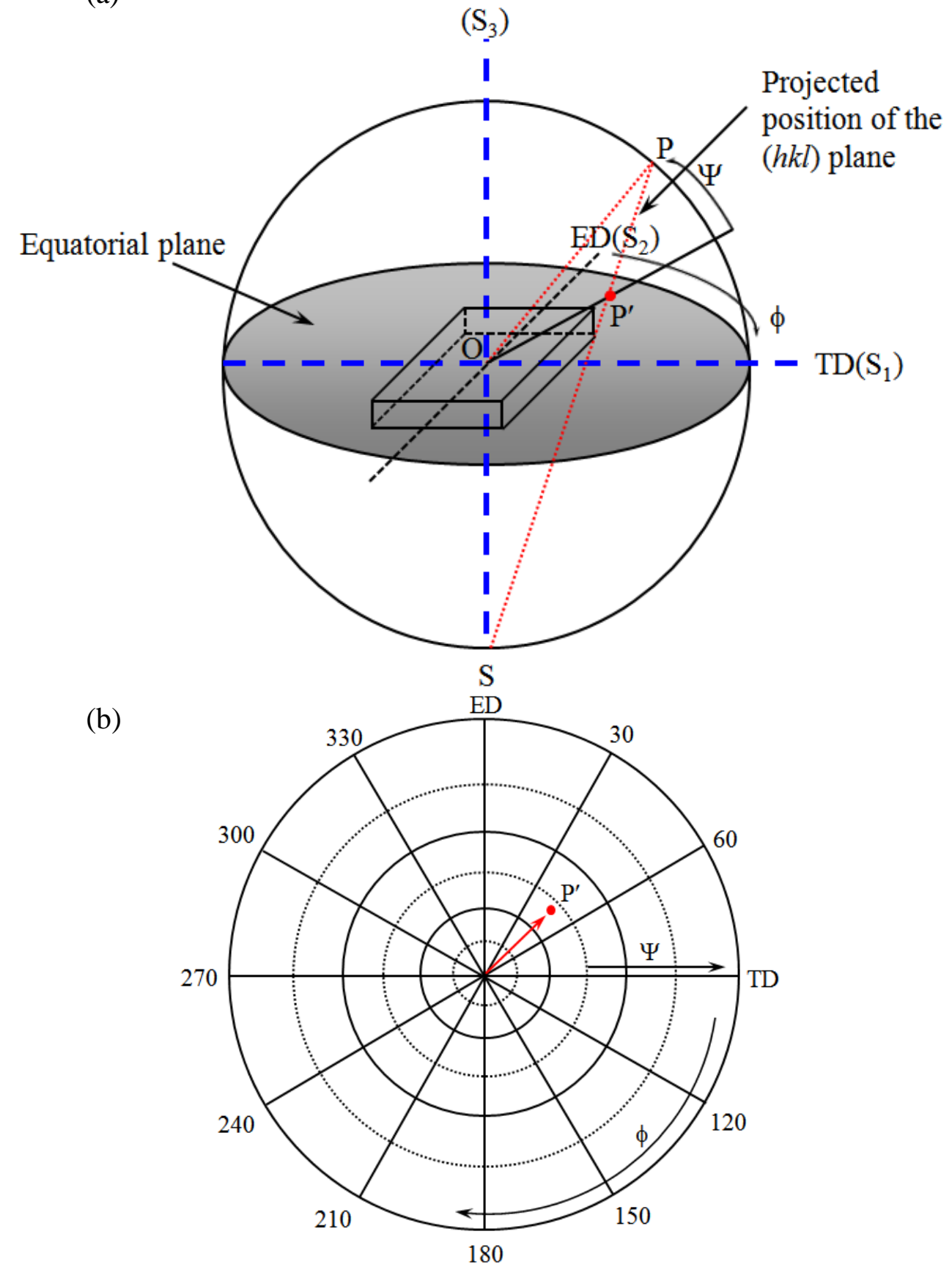

Figure 3.6: (a) Definition of pole direction angles $\phi$ and $\Psi$, (b) stereographic projection in a pole figure [90]. 
As shown in Fig. 3.6(b), this two-dimensional pole density mapping on the equatorial plane becomes a pole figure. The azimuthal angle $\phi$ projects to the pole figure as a rotation angle about the center of the pole figure from the sample direction $S_{2}$. The radial angle $\Psi$ is projected to the pole figure with a nonlinear scale.

Pole figures contain some partial data about the orientation-distribution function (ODF), which is a mapping of the probability of each of the possible grain orientations with respect to the macroscopic or sample coordinates. The ODF is defined as a probability density function of orientations, $g$, expressed, for example, in the form of the Euler angles $\varphi_{1}, \Phi$, and $\varphi_{2}$.

Let us assume a microstructure consisting of different grains $i$ of volume $V_{i}$ with different orientations as shown in Fig. 3.7. Grains of similar orientations $g$ within an orientation range $d g$ are color coded with the same gray value. The ODF, $f(g)$, is defined by the following relationship:

$$
\frac{d V}{V}=f(g) d g,
$$

with $d g=\frac{1}{8 \pi^{2}} \sin \Phi d \varphi_{1} d \Phi d \varphi_{2}$,

and $\oint f(g) d g=1$, where $V$ is the sample volume and $d V$ is the volume of all crystallites $i$ with the orientation $g$ in the angular element $d g$. 
In this study texture was determined using a PANalytical X-ray diffractometer (XRD) by measuring a set of five incomplete pole figures (\{0001\}, $\{10 \overline{1} 0\},\{10 \overline{1} 1\},\{11 \overline{2} 0\},\{10 \overline{1} 3\})$ between $\Psi=0$ to $75^{\circ}$ in a back reflection mode using $\mathrm{Cu} \mathrm{K}_{\alpha}$ radiation at $45 \mathrm{kV}$ and $40 \mathrm{~mA}$. Then the pole figures were evaluated using MTEX toolbox [117]. It should be noted that defocusing may occur due to the rotation of the XRD sample holder during the texture measurement, which was subsequently corrected using experimentally determined data from the diffraction of magnesium powders which were assumed to be texture-free.

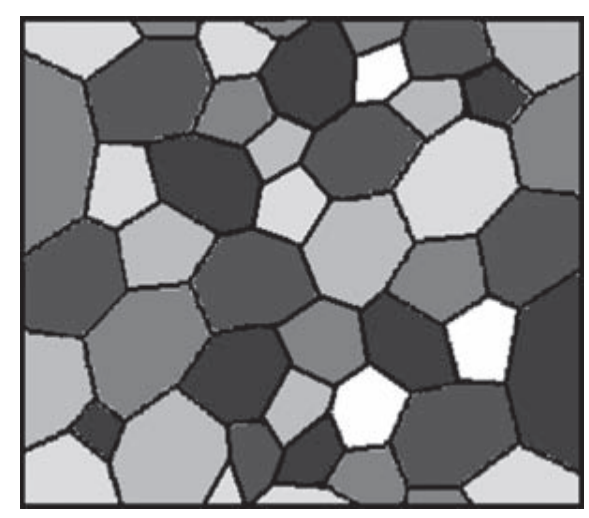

Figure 3.7: Schematic representation of a microstructure consisting of different grains $i$ of volume $V_{i}$ with different orientations. Similar orientations $g$ within an orientation range $d g$ is color coded with the same gray value [90].

In this dissertation, for compression tests, 2-3 samples were tested in each condition to check the reproducibility of the experiments. Either all the data points in each case or average value were plotted in the graphs. In the observations of the microstructure, several micrographs were taken for each experimental condition and then summarized. To ensure the accuracy of the analysis, 
texture was determined using five incomplete pole figures. To ensure the accuracy of the analysis, texture was determined using five incomplete pole figures. 


\section{CHAPTER 4}

\section{Twinning and Texture Development during Compression}

\subsection{Introduction}

As surveyed in Chapter 2, the twin formation would be dependent on the preferred orientation (texture) of grains with respect to the loading direction. For the wrought Mg alloys, most basal planes are aligned parallel to the extrusion or rolling direction, which would lead to the occurrence of extension twinning during compression along the extrusion or rolling direction [13-16,20,33,34]. However, it is unclear how the twinning and their growth occur with increasing strain, and how they affect the strain hardening behavior. Also, it is unknown how the texture components present in an extruded AM30 alloy change during compression. The purpose of this study was, therefore, to identify the twinning and their growth in an extruded Mg alloy in relation to the strain level and strain hardening characteristics during compressive deformation, with particular attention to identify the texture components and their evolution with increasing compressive strain.

\subsection{Initial Microstructure}

The microstructure of the as-extruded material is shown in Fig. 4.1(a), consisting of a mix of large and small grains which were twin-free. The initial texture of this material, shown in Fig. 4.1(b), exhibited a strong basal texture with a maximum intensity of 8.6 multiples of random distribution (MRD). While the basal (0001) poles had about $20^{\circ}$ tilt towards the ED, the (1010) 
poles were observed to tilt towards both the ED and TD, indicating that two sets of initial basal textures, i.e., $\{0001\}<2 \overline{1} \overline{1} 0>$ and $\{0001\}<10 \overline{1} 0>$, were present in the extruded AM30 Mg alloy. Ma et al. [107] studied the texture evolution in an extruded Mg-Al-Mn alloy (AM30) during uniaxial compression at $450^{\circ} \mathrm{C}$ and under various strain rates. In compression along the extrusion direction (ED) and with strain rates of greater than $0.5 \mathrm{~s}^{-1}$, resulted in two texture components $<10 \overline{1} 0>\| \mathrm{ED}$ and $<2 \overline{1} \overline{1} 0>\| \mathrm{ED}$, with an angle of $30^{\circ}$ between these components. Kun et al. [16] also reported the evolution of texture during the annealing and hot rolling process of extruded AZ31 magnesium alloy sheets, and described two kinds of texture components $\{0002\}<10 \overline{1} 0>$ and $\{10 \overline{1} 0\}<11 \overline{2} 0>$. Based on the present results, a schematic diagram could be plotted in Fig. 4.1(c), where the $c$-axes of hcp unit cells in most grains were nearly perpendicular to the ED in both cases of cylindrical and rectangular samples.

\subsection{Twin Growth Behavior}

Fig. 4.2(a) shows a typical microstructure of the as-extruded AM30 magnesium alloy, where a twin free grain is magnified. As the alloy exhibited strong basal textures with the $c$-axes aligned almost parallel to the ND (Fig. 4.1(b)), after compression to a true strain amount of $1.7 \%$ along the ED, several twins with varying widths were formed as shown in Fig. 4.2(b). With increasing compressive strain, the growth of twins was clearly observed. When the stepwise compression reached a cumulative true strain of $4.4 \%$, widening of the previous twins occurred (Fig. 4.2(c)). A further increase of strain to $9.9 \%$ led to more twin growth and coalescence (or the disappearance of twin boundaries), as shown in Fig. 4.2(d). 

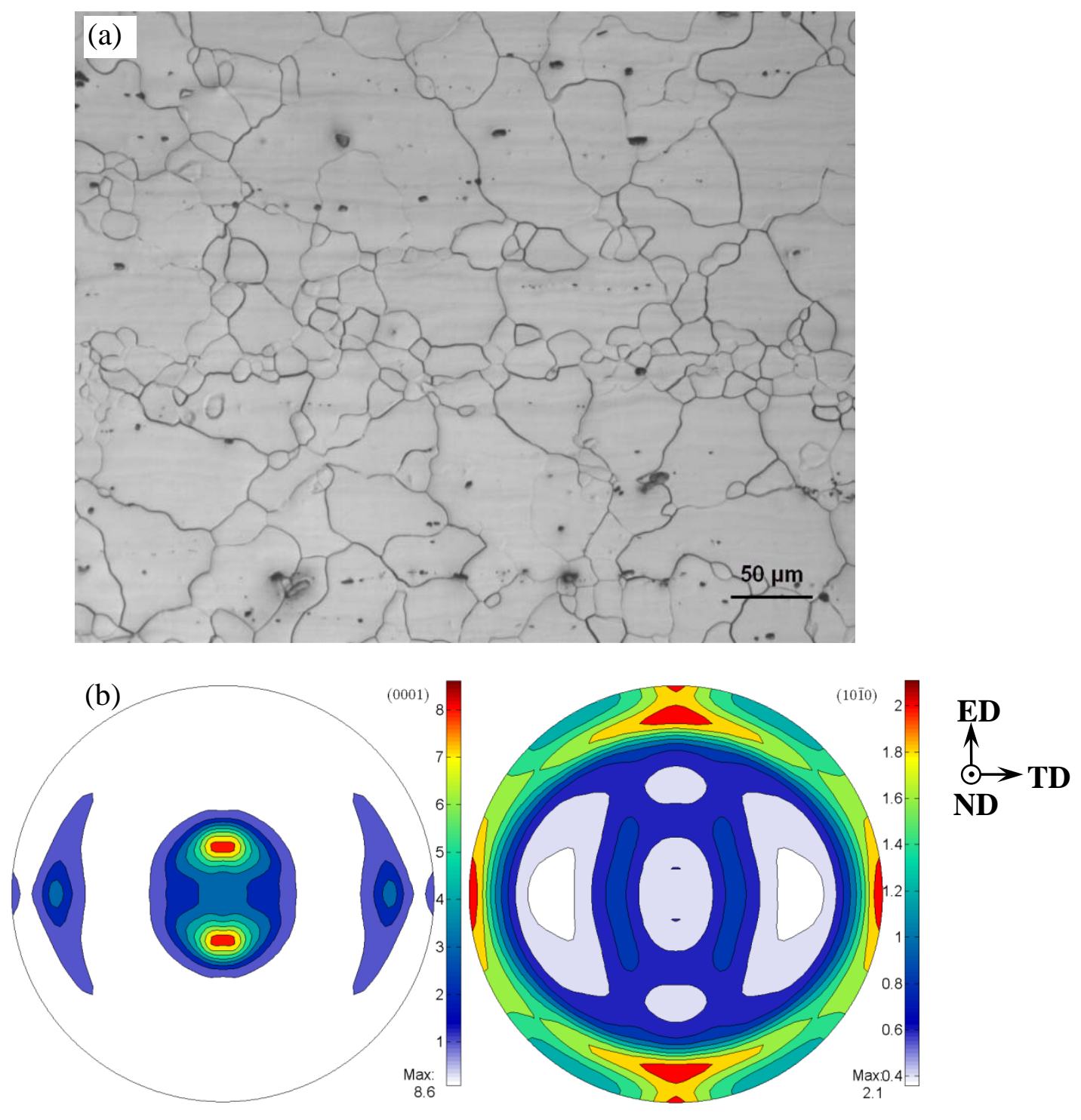

(c)

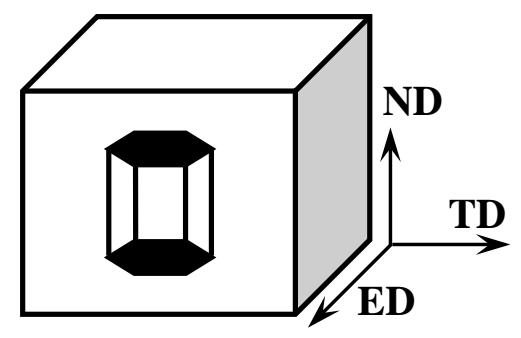

Figure 4.1: (a) Microstructure, (b) (0001) and (1010) pole figures of the extruded AM30 magnesium alloy, (c) schematic diagrams illustrating the relationship between the $c$-axis of most grains and the compression axis oriented horizontally. 
The growth and coalescence of twins eventually encompassed the whole grain, indicative of complete twin growth. The formation and growth of the twins observed were due to the fact that the extruded magnesium alloy contained strong basal textures with the $c$-axes of hcp unit cells in most grains being oriented perpendicular to the ED (Fig. 4.1(b)). During compression along the ED the nucleation and growth of $\{10 \overline{1} 2\}$ extension twinning would occur easily due to the low CRSS. These observations were consistent with those reported in the literature.

Hong et al. [118] reported that twins could nucleate at a fairly low strain of about 2\%, and twin growth could occur at a strain between $2 \%$ and $6 \%$, and then coalesce. Twin growth occurred mainly due to the interaction of dislocations with twin boundaries, depending on the characteristics of dislocations and the driving force [32,118,119].

To quantify the twin growth behavior, the change of the width of a few representative twins indicated as "A", "B" and "C" in Fig. 4.2 as a function of cumulative true strain was measured after each step of compression and plotted in Fig. 4.3. After the initial compression at a strain of 1.7\%, the width of twins A, B and C were $5.2 \mu \mathrm{m}, 6.6 \mu \mathrm{m}$ and $1.3 \mu \mathrm{m}$ respectively. With increasing compressive true strain from $1.7 \%$ up to $9.9 \%$, the width of all three twins clearly increased. It is of interest to note that for all the twins, the width vs. strain curve exhibited a sigmoid shape with three distinct stages. Stage I is characterized by a relatively slow growth rate with respect to strain (i.e., the slope $d W / d \varepsilon$ in Fig. 4.3 , where $W$ is the twin width and $\varepsilon$ is the cumulative true strain). Stage II exhibits a linear increase of twin width with increasing strain at a constant growth rate. This suggests that the twin growth reaches a steady state. The twin growth in stage III slows down again, as seen from Fig. 4.3 where all curves display a plateau- 

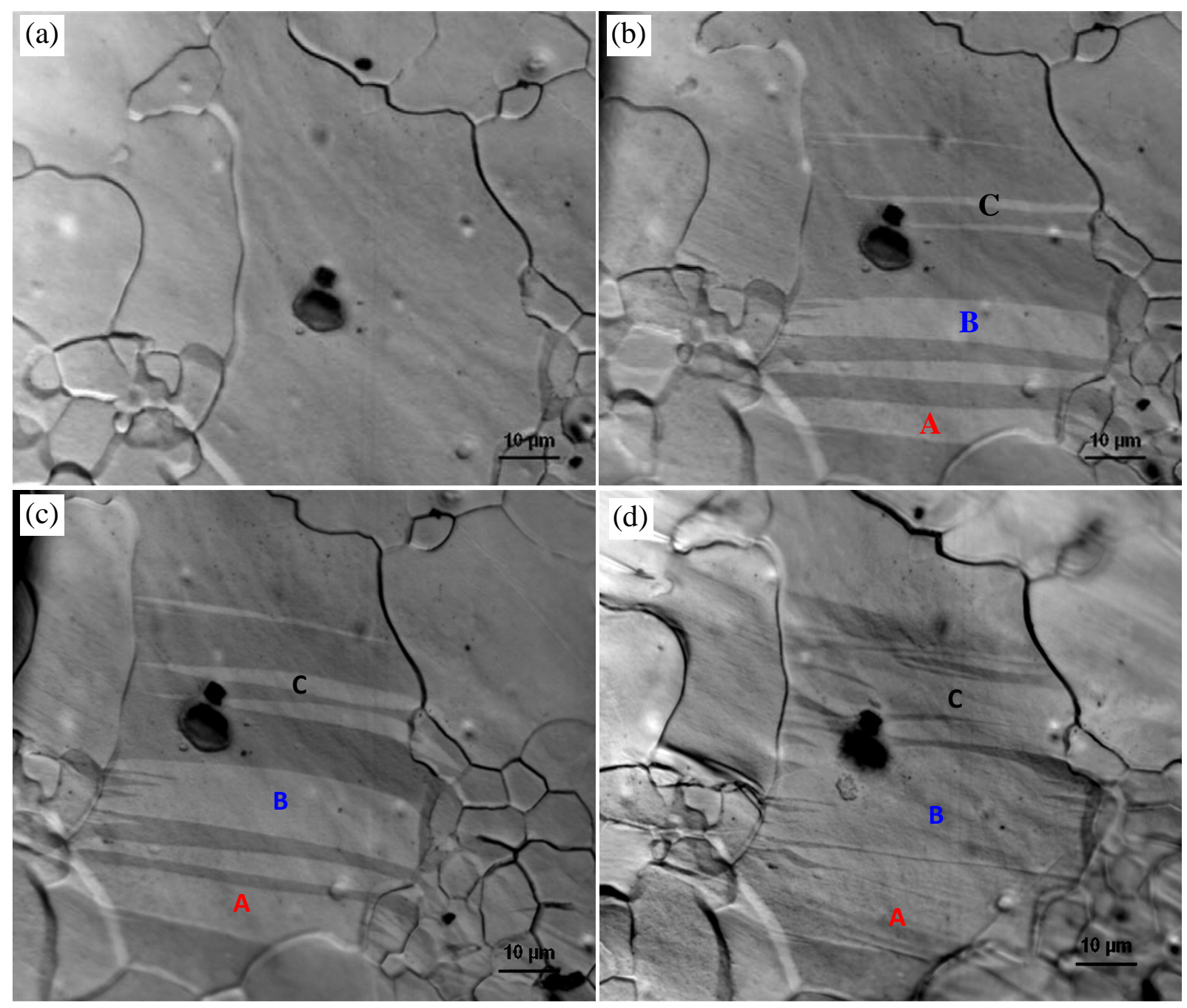

Figure 4.2: (a) Initial twin-free microstructure and twin growth during stepwise compression after a cumulative true strain amount of (b) $1.7 \%$, (c) $4.4 \%$, and (d) $9.9 \%$ along the ED.

-like character. This is understandable, since the growth of twins approaches their completion in this stage. It is also interesting to note that the steady-state growth rate (or the slope in stage II) was different, where the initially wider twins grew faster.

To describe the twin growth mechanism, schematic diagrams are plotted in Fig. 4.4, where $\{10 \overline{1}$ 2\} extension twins are formed and embedded within a grain, which is normally activated 


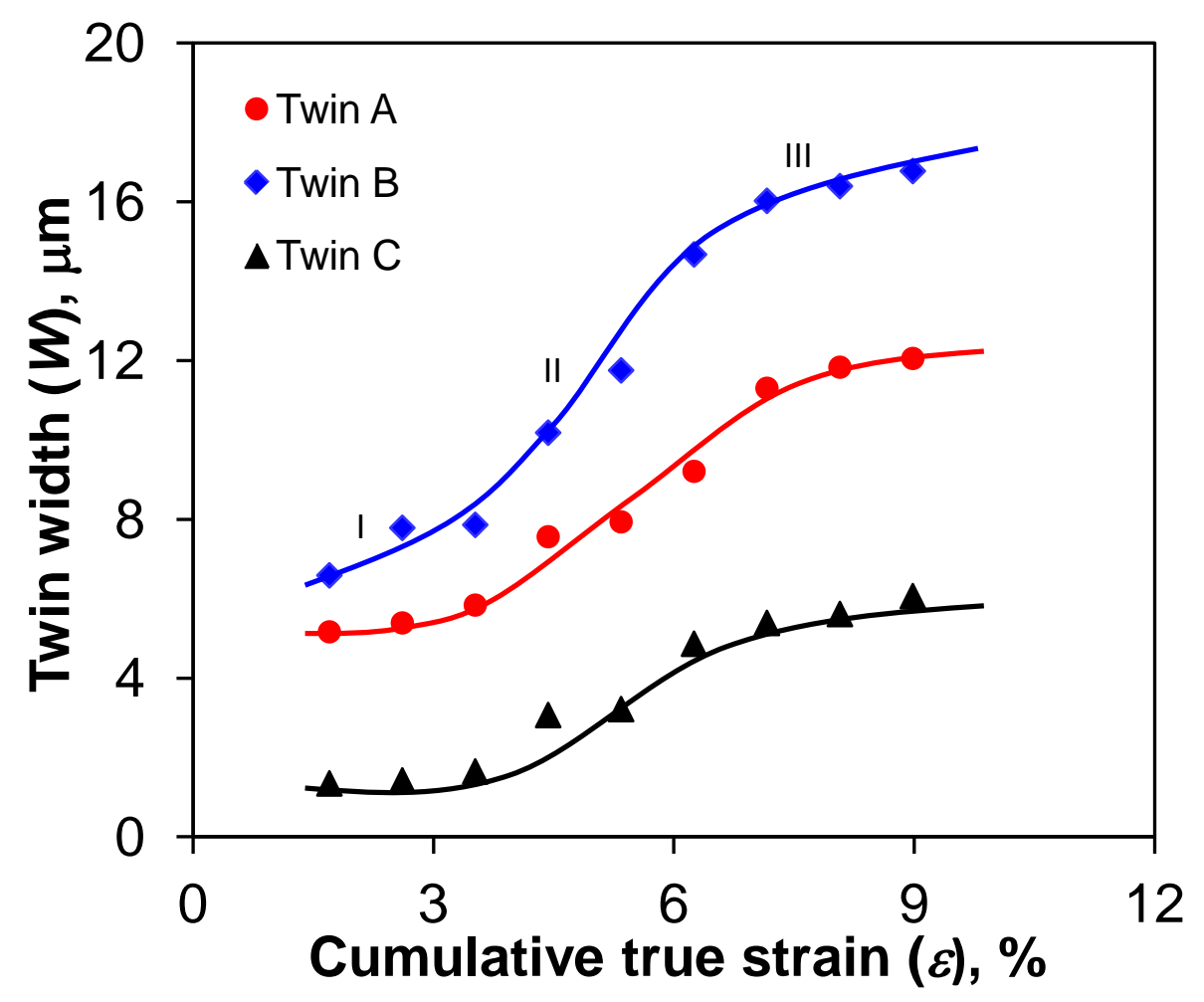

Figure 4.3: Twin width vs. cumulative true strain after stepwise compression.

when a compressive stress is applied perpendicular to the $c$-axis, i.e., along the ED as shown in Fig. 4.4(a). The occurrence of $\{10 \overline{1} 2\}$ extension twins led to an $86.3^{\circ}$ rotation of the $c$-axis in the twinned regions with respect to the $c$-axis in the matrix [19], as indicated in Fig. 4.4(a).

This means that the $c$-axes of hcp unit cells in the twinned regions were always rotated towards the compression direction or aligned along the loading axis (i.e., ED in this case). With continuing compression along the same loading direction, twins started to grow as shown in Fig. 4.2(b) to (d). In the AM30 magnesium alloy twin-twin interactions during their nucleation and propagation were observed by Kadiri et al. [120] as well, and they reported that twinning was not observed in any region of the sample until the plastic strain reached $1.3 \%$, and beyond appr- 

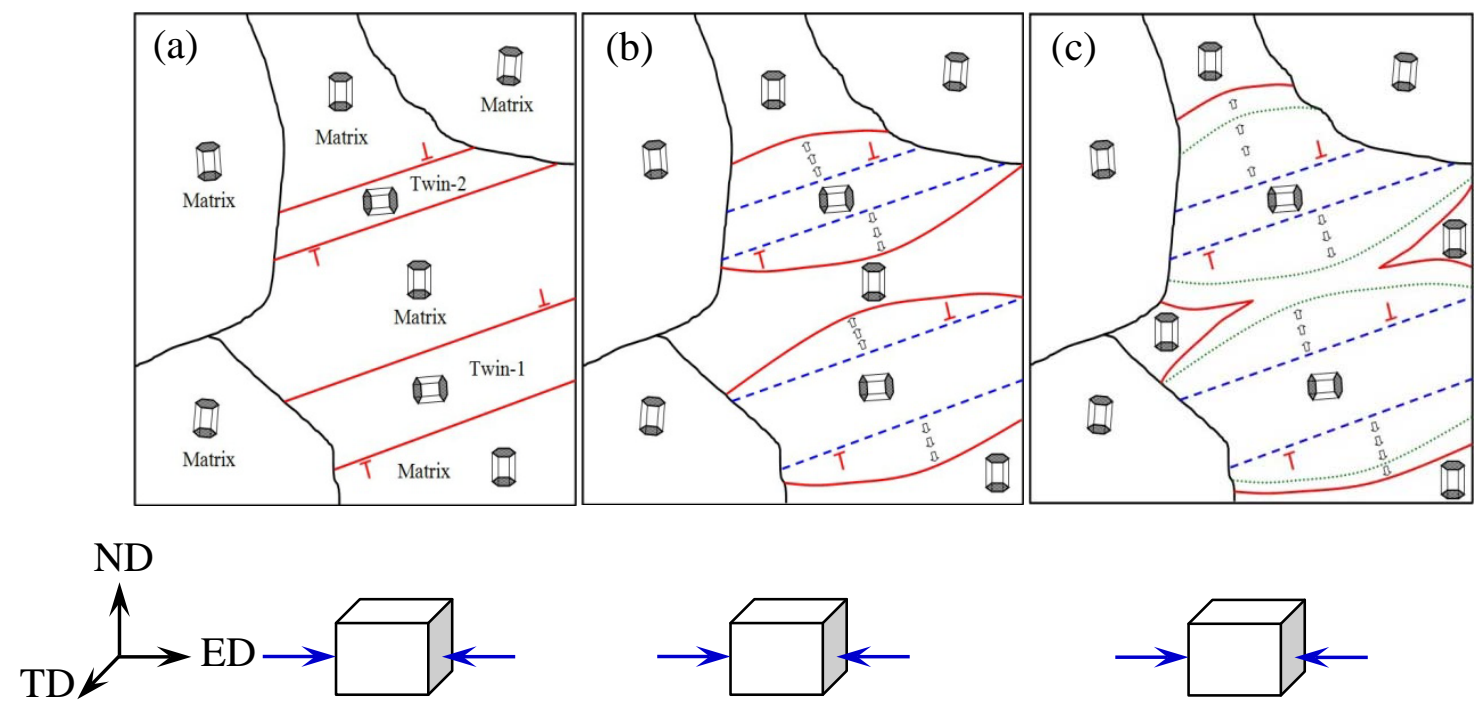

Figure 4.4: Schematic illustration of the growth of $\{10 \overline{1} 2\}$ extension twins in a grain: (a) formation of twins inside a grain by rotating basal planes at an angle of $86.3^{\circ}$, (b) twin growth, (c) coalescence of twins (or vanishing of twin boundaries) to continue twin growth.

-oximately $8 \%$ strain grains were completely consumed by $\{10 \overline{1} 2\}$ extension twinning. Twindislocation interactions play a key role in the twin growth. In the dislocation theory, upon meeting with a $\{10 \overline{1} 2\}$ twin boundary, a slip dislocation can penetrate into the twin and pass through the other side of the twin boundary (TB), undergo a core reconstruction, or dissociate into interfacial defects [121].

Twin growth occurs when the dissociation products are glissile twin dislocations (TDs) that glide through the TB in the matrix, where the driving force is the stress acting on TDs at the TB interfaces $[86,121,122]$. When the shear stress along the twin boundary is larger than the CRSS of twin propagation, a twin can grow [123]. However, the high local stress concentration at the twin ends (i.e., at the intersection of a twin with grain boundaries) requires more driving force 
for the twin to grow near the ends than in the middle. Hence, the twin can keep growing in the middle area with a lesser resistance compared with its growth near the two ends, leading to a lenticular shape as schematically shown in Fig. 4.4(b) and generally observed, e.g., in [124-126]. The mobility of twin boundaries during compression was also observed by Nie et al. [3], who reported the formation of twins after the first compression of a Mg-Gd alloy to a strain of $2.5 \%$. After the specimen was re-compressed to an accumulated strain of $4.5 \%$, they detected further growth of twins generated during the first compression. Further growth of twins by merging twin boundaries may cause coalescence of twins as shown in Fig. 4.4(c) and thus encompass the whole grain.

\subsection{Role of Twinning on Strain Hardening}

The stress-strain curve and strain-hardening rate of AM30 magnesium alloy during compression along the ED is shown in Fig. 4.5(a) and (b), respectively, where the stress-strain curves of several samples deformed by varying amounts are plotted in Fig. 4.5(a) as well. Good reproducibility was seen, and a compressive yield strength of $71 \pm 3 \mathrm{MPa}$ was obtained, which was considerably lower than the tensile yield strength of 189 MPa [15], due to the occurrence of twinning during compression. The stress-strain curve of AM30 extruded alloy exhibited a skewed shape (Fig. 4.5(a)), which is not usually seen in the normal tensile (or compressive) curves of most metals with cubic crystal structures.

This could be better seen based on the change of strain hardening rate $d \sigma / d \varepsilon$ (where $\sigma$ is true stress) with respect to true strain, $\varepsilon$, where three stages of strain hardening can be more clearly 

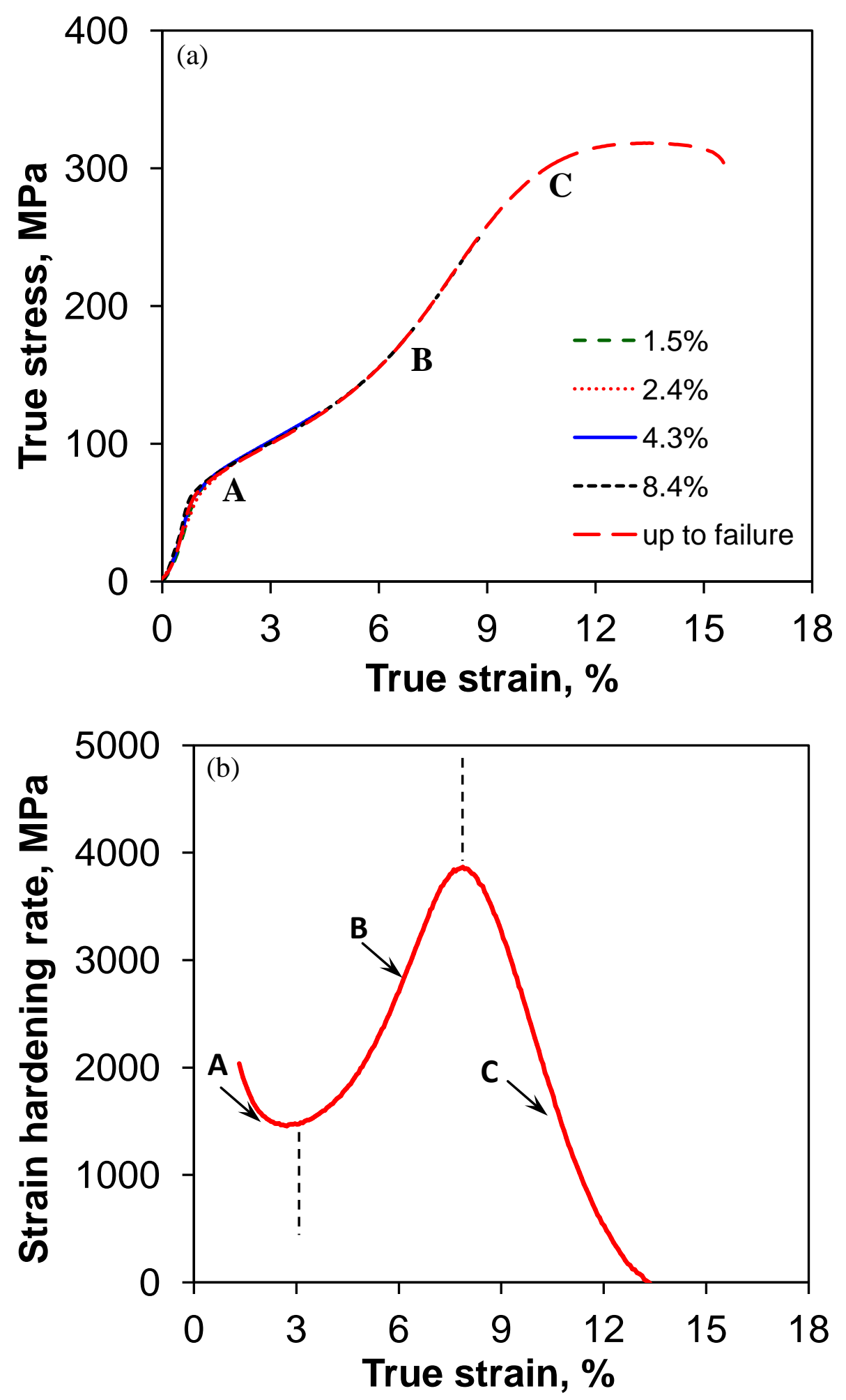

Figure 4.5: (a) Compressive true stress-true strain curves of extruded AM30 magnesium alloy deformed to different strain levels, and (b) strain hardening rate as a function of true strain. 
distinguished (Fig. 4.5(b)). Stage A was characterized by a decreasing strain hardening rate up to approximately 3\% compressive true strain, followed by stage B with an increasing strain hardening rate up to $\sim 8.0 \%$ compressive true strain, and then stage $\mathrm{C}$ with decreasing strain hardening rate again until failure. A similar trend in the strain hardening rate has also been observed in $\alpha$-titanium [127] and AZ31 magnesium alloys [128].

To understand such a peculiar change, the deformation characteristics of the samples compressed to varying strain amounts were examined. Fig. 4.6 shows the feature of twins as a function of strain. Comparing Fig. 4.6 with Fig. 4.5, it is seen that stage A deformation after yielding at about 70 MPa was characterized by a decreasing strain hardening rate (Fig. 4.5(b)) and increasing amount of twin with increasing strain up to nearly 3\% compressive strain (Fig. 4.6). At a compressive strain of $\varepsilon=1.5 \%$, a small number of lenticular shaped extension twins were observed in some large grains (Fig. 4.6(a)), whereas at $\varepsilon=2.4 \%$ more and thicker twins were observed (Fig. 4.6(b)). Similar results have been reported in the wrought AZ31B-H24 Mg alloy [34], where the flow stress after compressive yielding remained nearly constant until the maximum compressive strain reached at $3.0 \%$, and the volume fraction of extension twinning reached the maximum. It is of particular interest to observe that stage B strain hardening was represented by an increasing strain hardening rate (Fig. 4.5(b)) and decreasing the existence of twin boundaries (Fig. 4.6) in the compressive strain range in-between $\sim 3 \%$ and $\sim 8 \%$. It should be pointed out that such a stage of increasing strain hardening rate was absent in the wellestablished strain hardening characteristics of cubic metals. At such higher strain levels it was likely that the dislocation slip would be activated. 

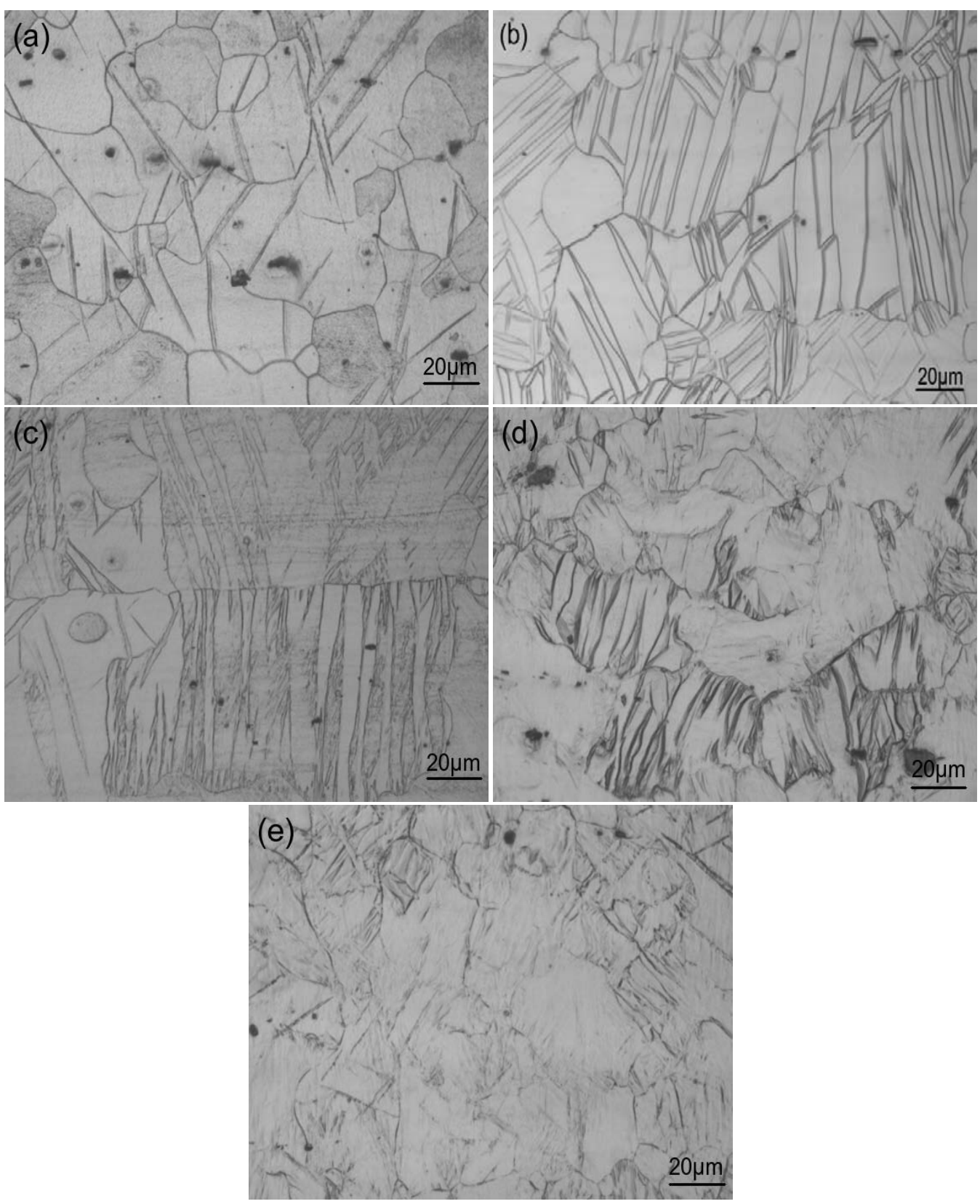

Figure 4.6: Optical micrographs at different strain levels of, (a) 1.5\%, (b) $2.4 \%$, (c) 4.3\%, (d) 8.4\% and (e) 12.9\% during compression of extruded AM30 magnesium alloy.

The increasing strain hardening rate mainly arose from the interaction of dislocations with twins generated in stage A, which caused the starting of twin growth as observed in Fig. 4.2. The 
phenomena of twin growth with increased compressive strain merged their boundaries (Fig. 4.4) as seen from images (c) and (d) of Fig. 4.6. As seen in Fig. 4.6(c), some twin boundaries in some grains were observed to start to fade away after a strain of $\sim 3 \%$. At a higher strain of $\varepsilon=$ 8.4\% more twin boundaries disappeared (Fig. 4.6(d)).

In stage C, with a compressive true strain beyond $\sim 8.0 \%$, the strain hardening rate (Fig. $4.5(\mathrm{~b})$ ) decreased, whereas the disappearance of twin boundaries (Fig. 4.6) increased. As seen from Fig. 4.6(e), twin boundaries disappeared in most grains at a compressive strain of $\varepsilon=12.9 \%$, indicating complete twin growth in stage C.

\subsection{Texture Transformation during Compressive Strain}

The change of texture during compression is shown in Fig. 4.7 for several strain levels. At a strain of 1.5\%, basal (0001) poles showed some extent of split of intensities from the center towards the radial direction (RD) with weak intensities along the ED, while prismatic (1010) poles with a moderate intensity were oriented towards the ED, as shown in Fig. 4.7(a).

More rotations of basal planes towards the ED and prismatic planes towards the center were obvious with increasing strain levels from $1.5 \%$ to $4.3 \%$ to $8.4 \%$ (Fig. 4.7(a) to (c)). The basal planes of further more grains were rotated to ED after $12.9 \%$ strain as indicated by the increasing maximum intensity from 12 to $16 \mathrm{MRD}$, while the prismatic planes were oriented inbetween the center and RD, as shown in Fig. 4.7(d). 
(a)
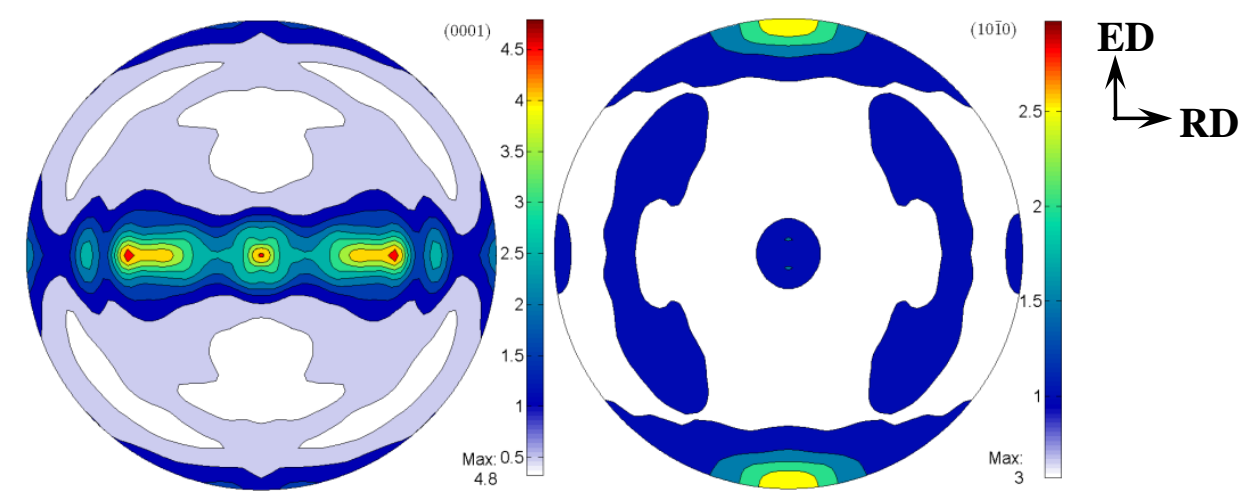

(b)

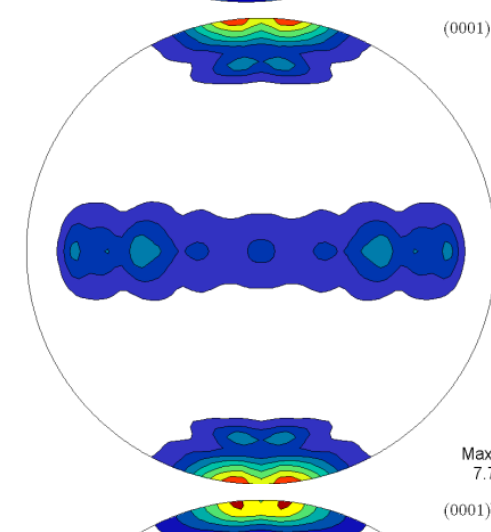

(c)

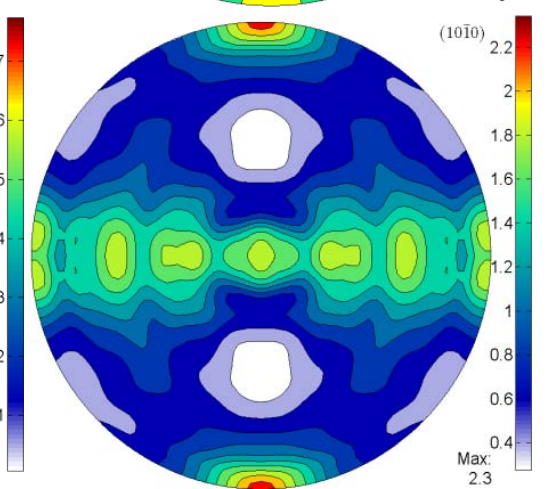

(d)

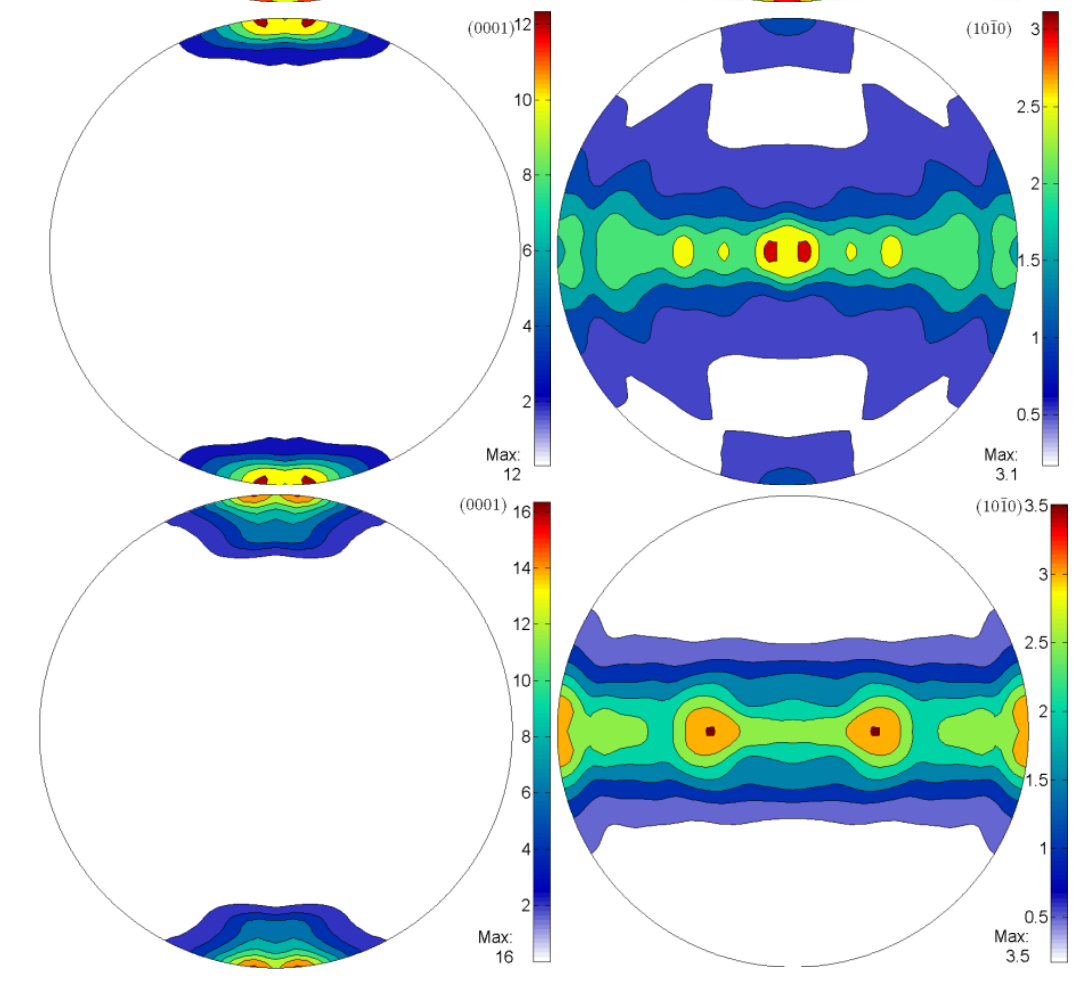

Figure 4.7: (0001) and (1010) pole figures obtained from the compressed samples at a strain amount of (a) $1.5 \%$, (b) $4.3 \%$, (c) $8.4 \%$, and (d) $12.9 \%$. 
Table 4.1: Main texture components identified from Euler angles.

\begin{tabular}{|c|c|}
\hline $\begin{array}{c}\text { Euler angles } \\
\left(\varphi_{1} \Phi \varphi_{2}\right)\end{array}$ & $(h k i l)[u v t w]$ \\
\hline $\mathrm{A}(90,20,0)$ & $\{0001\}<2 \overline{1} \overline{1} 0> \pm 20^{\circ}$ towards ED \\
\hline $\mathrm{B}(90,20,30)$ & $\{0001\}<10 \overline{1} 0> \pm 20^{\circ}$ towards ED \\
\hline $\mathrm{C}(90,90,0)$ & $\{\overline{1} \overline{1} 0\}<0001>$ \\
\hline $\mathrm{D}(90,90,30)$ & $\{01 \overline{1} 0\}<0001>$ \\
\hline
\end{tabular}

To better understand the evolution of texture components, ODF was presented in a Euler space of $0^{\circ} \leq \varphi_{1} \leq 90^{\circ}, 0^{\circ} \leq \Phi \leq 90^{\circ}$ and $0^{\circ} \leq \varphi_{2} \leq 60^{\circ}$ as the alloy has hexagonal crystal symmetry. The texture change during compression at strain amounts of $0 \%, 4.3 \%$ and $12.9 \%$ is shown in Fig. 4.8(a), represented by the ODF sections at $\varphi_{2}=0^{\circ}$ and $\varphi_{2}=30^{\circ}$ from which the main texture components in hcp materials could be distinguished $[22,23,29]$. In the ODF sections several major components marked as A, B, C and D can be clearly identified, with the corresponding Euler angles given in Table 4.1.

The undeformed alloy showed two types of basal texture components $\{0001\}<2 \overline{1} \overline{1} 0>$ at orientation $\mathrm{A}(90,20,0)$ and $\{0001\}<10 \overline{1} 0>$ at orientation $\mathrm{B}(90,20,30)$, with their basal planes being $\pm 20^{\circ}$ towards the ED as indicated by $\Phi=20^{\circ}$ while $\varphi_{1}=90^{\circ}$ [129]. A salient change in ODF at different strain levels was observed with respect to the undeformed alloy (Fig. 4.8(a)). That is, both texture components $\mathrm{A}\{0001\}<2 \overline{1} \overline{1} 0>$ and $\mathrm{B}\{0001\}<10 \overline{10}>$ disappeared, and were replaced by the formation of texture components $C\{\overline{1} 2 \overline{1} 0\}<0001>$ and $\mathrm{D}\{01 \overline{1} 0\}<0001>$ at orientations $(90,90,0)$, and $(90,90,30)$, respectively, with increasing strain up to $12.9 \%$. 


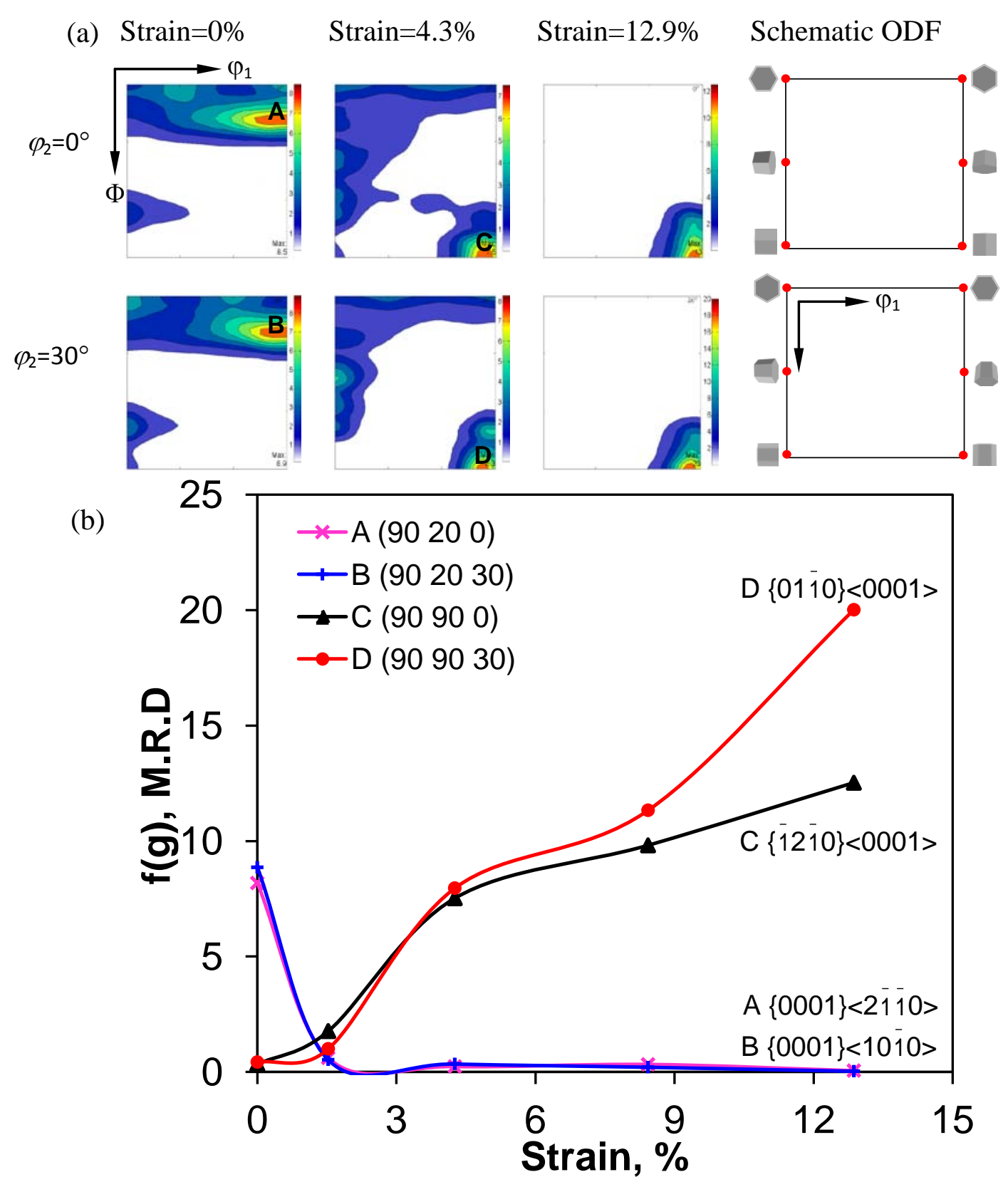

Figure 4.8: (a) ODF sections at $\varphi_{2}=0^{\circ}$ and $\varphi_{2}=30^{\circ}$, from the samples strained at $0 \%, 4.3 \%$ and $12.9 \%$, and (b) the change of intensity of main texture components with the strain.

The change of components A to C in the ODF section of $\varphi_{2}=0^{\circ}$ and components B to D in the section of $\varphi_{2}=30^{\circ}$ with increasing strain indeed reflected a rotation of $c$-axes from an 
orientation of $\pm 20^{\circ}$ towards the ED to parallel to the ED. This was equivalent to rotating the $c$ axes of most grains against the "push". To quantify the change of these texture components, their intensity (f(g)) as a function of compressive strain was evaluated and plotted in Fig. 4.8(b). Clearly, components A and B rapidly faded away, while components C and D increasingly intensified. In the later stages of compressive deformation, the intensity of component $\mathrm{D}$ became higher than that of component C.

This is likely related to the slightly higher density of component B (8.8 MRD), as compared with that of component A (8.2 MRD), since component B subsequently changed to component D during compressive deformation. Similar results in an extruded AZ31 alloy were also reported in [22], where the intensity of component D was higher than that of component C.

The texture volume fraction for the transformed $\{\overline{1} 2 \overline{1} 0\}<0001>$ and $\{01 \overline{1} 0\}<0001>$ components during compression could be quantified on the basis of the following equation [130-132], and is shown in Fig. 4.9:

$$
f_{X}=\frac{M_{X}-M_{o}}{M_{f}-M_{o}},
$$

where $f_{x}$ is the texture volume fraction at a particular strain $(x), M_{o}$ is the initial orientation density, $M_{x}$ is the orientation density at a strain $x, M_{f}$ is the final orientation density.

Before the compression tests (i.e., at $0 \%$ strain), the texture volume fractions of both $\{\overline{1} 2 \overline{1} 0\}<0001>$ and $\{01 \overline{1} 0\}<0001>$ components were zero, which increased with increasing strain as shown in Fig. 4.9. For example, at a strain to 4.3\% (Fig. 4.9), the texture volume 
fraction of $\{\overline{1} 2 \overline{1} 0\}<0001>$ and $\{01 \overline{10}\}<0001>$ components increased to $\sim 0.59$ and $\sim 0.38$, respectively. With a further increase of strain to $8.4 \%$, the texture volume fraction of $\{\overline{12} \overline{1}$ $0\}<0001>$ and $\{01 \overline{1} 0\}<0001>$ components increased to $\sim 0.78$ and $\sim 0.56$, respectively, and $\{\overline{1} 2 \overline{1}$ $0\}<0001>$ component became $\sim 1.0$ at a strain of $12.9 \%$. A $30^{\circ}$ angle between $\{\overline{1} 2 \overline{1} 0\}<0001>$ and $\{01 \overline{1} 0\}<0001>$ components indicated that slip may cause transformation of $\{\overline{1} 2 \overline{1} 0\}<0001>$ to $\{01 \overline{10}\}<0001>$ components which was also evident from the increased rotations of the basal (0001) poles towards the ED with an intensity of 12 MRD to 16 MRD (Fig. 4.7).

The observed change in the texture components can give us useful information about the deformation mode during compression. Based on the assumption that the intensifying of a texture component is at the expense of some other components, the development of components $\mathrm{C}$ and $\mathrm{D}$ can be associated with the weakening of components A and B. Such a rapid and large orientation change cannot be achieved by slip, but is possible by twinning $[22,23,133,134]$. The $\{10 \overline{1} 2\}$ extension twinning has been observed to play a predominant role in the texture evolution in the deformation of magnesium alloys [133,134], which was also responsible for the tension and compression anisotropy $[13,14,16]$. The initial components A and B with the $c$-axes of most grains nearly perpendicular to the ED (Fig. 4.1(b) and Fig. 4.8(a)) were favorably oriented for the activation of extension twinning when compressed along the ED (Fig. 4.1(c)). The occurrence of this type of twinning induces an $86.3^{\circ}$ rotation of the basal plane or $c$-axis [19], so that the activation of $\{10 \overline{1} 2\}$ twinning would satisfy the large orientation change of texture components $\mathrm{A} \rightarrow \mathrm{C}$ and $\mathrm{B} \rightarrow \mathrm{D}$. The occurrence of abundant extension twinning in the extruded magnesium alloys, e.g., AM30, has been observed in [15,135,136]. 


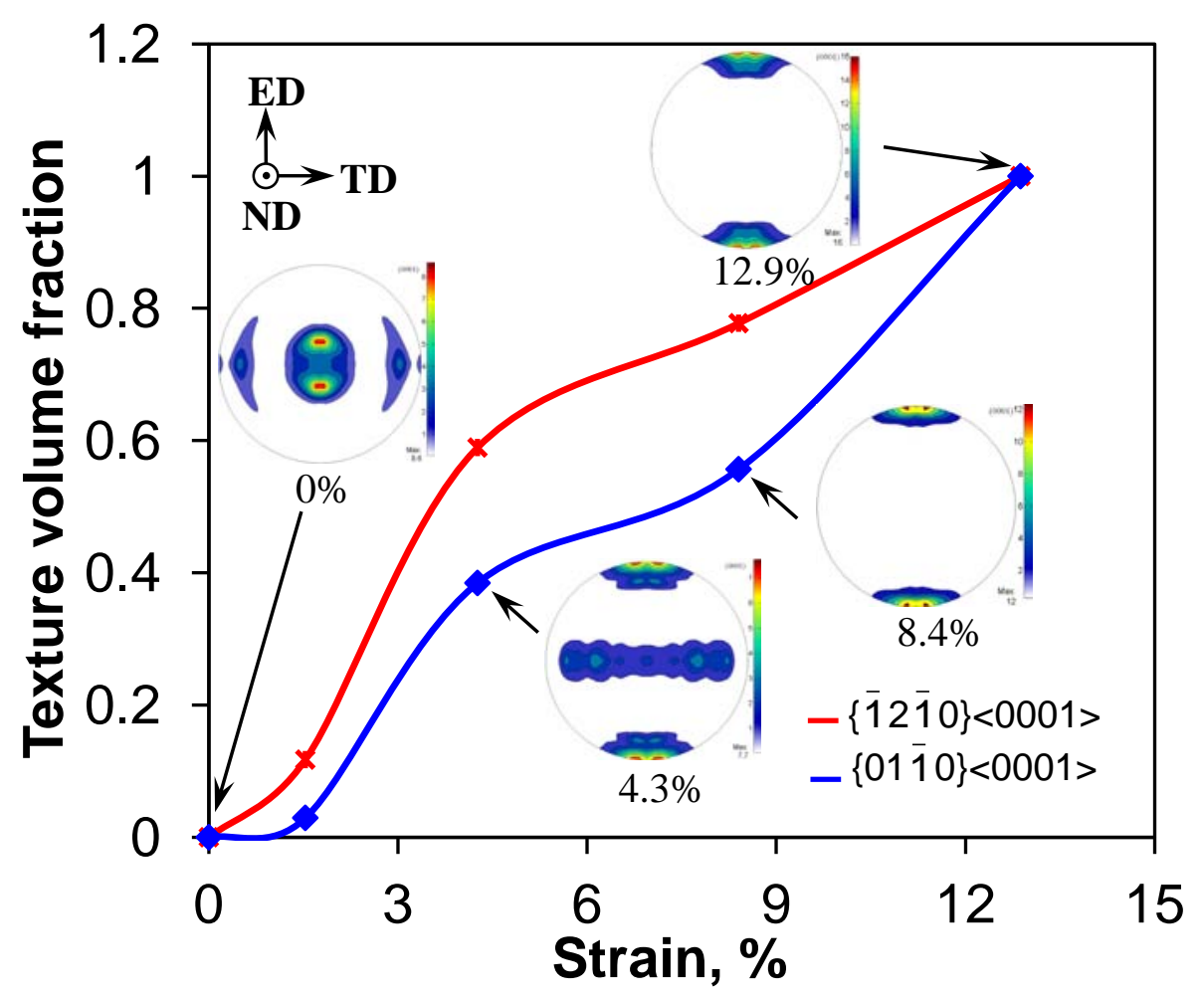

Figure 4.9: Texture volume fraction vs. strain after compression.

Therefore, the evolution of texture components in the extruded magnesium alloy is predominantly related to the twinning. Additionally, as reported in $[22,23,29]$, since the interplanar angle between components $\mathrm{C}$ and $\mathrm{D}$ is $30^{\circ}$, the rotation of the $c$-axes of most grains towards the compression was anticipated to be also associated with the dislocation glide involving the twin-dislocation interactions and twin-twin interactions during deformation $[135,136]$. Further studies in this aspect are needed, e.g., by using atomic scale modeling and insitu high-resolution transmission electron microscopy to observe the migration of twin boundaries during plastic deformation [137]. 


\subsection{Summary}

In this chapter, the deformation behavior of an extruded AM30 alloy during compression along the extrusion direction at different strain levels was studied, and the experimental results regarding the extent of twinning and twin growth, strain hardening characteristics during compression, with particular attention to texture components with increasing compressive strain, were presented and discussed.

Extension twins were introduced at an initial strain of $1.7 \%$ during compression along the ED and a gradual twin growth with increasing cumulative true strain along the same loading direction was observed. A sigmoid shape in the curves of twin width vs. cumulative true strain was observed and three stages of twin growth were identified. Stage I was characterized by a relatively slow growth rate with respect to the cumulative true strain but with an accelerating trend. In stage II the twin growth reached a steady state with a constant growth rate (or slope $d W / d \varepsilon$ ), where the twin width increased linearly with increasing strain. The twin growth in stage III decelerated, exhibiting a plateau-like character due to the fact that the growth of twins approached their completion. Furthermore, the steady-state growth rate was observed to be higher for the initially wider twins.

A sigmoid shape in the true stress-true strain curve is observed with three distinct stages of strain hardening behavior where stage A corresponded to a decreasing strain hardening rate up to a strain level of $\sim 3 \%$, followed by stage $B$, an increasing strain hardening rate over an 
extended strain range from $3 \%$ to $8 \%$. Beyond this strain level, stage C was reflected by an almost linearly decreasing strain hardening rate.

The present study demonstrates that the compressive deformation along the ED in AM30 extruded magnesium alloy led to a remarkable change in texture components. Both types of initial basal texture components $\{0001\}<2 \overline{1} \overline{1} 0>$ and $\{0001\}<10 \overline{1} 0>$ were observed to fade away, while $\{\overline{1} 2 \overline{1} 0\}<0001>$ and $\{01 \overline{1} 0\}<0001>$ texture components intensified with increasing compressive strain. Such texture changes reveal that the $c$-axes of hcp unit cells of most grains inside the material were always rotated towards "push" or compression direction, mainly due to their $86.3^{\circ}$ re-orientations stemming from extension twinning. 


\section{CHAPTER 5}

\section{Effect of Crystallographic Orientation and Loading Direction on Schmid Factor}

\subsection{Introduction}

The hexagonal close-packed (hcp) crystal structure of magnesium possesses limited slip systems, while five independent slip systems are required for homogeneous deformation according to Taylor criteria [138,139]. Moreover, in magnesium the critical resolved shear stress (CRSS) for most slip deformation modes, except the basal slip, is higher than that of twinning [140], which consequently makes twinning essential in the deformation at room temperature [141]. This twinning, in turn, is a function of the $c / a$ ratio, available deformation modes, critical resolved shear stress (CRSS) for slip, twin activation stress, and the imposed deformation gradient matrix along with its orientation related to the crystallographic texture [142]. The presence of crystallographic textures in wrought magnesium alloys, which are developed during manufacturing processes (extrusion, rolling, forging, etc.), causes poor room temperature formability and anisotropy in mechanical properties.

Recently, there have been efforts to weaken the intense basal texture of wrought magnesium alloys by adding rare earth elements, and/or applying various process methods [143-145]. Mukai et al. [146] showed that an AZ31 alloy processed by a shear extrusion technique (equal channel angular extrusion (ECAE) exhibits a significant enhancement in room temperature ductility. The ductility enhancement of the ECA-extruded $\mathrm{Mg}$ alloy is related to an unusual texture, where the 
basal planes are highly inclined $\left(45^{\circ}\right)$ to the extrusion direction axis [147-149]. Chino et al. [150] also observed enhanced ductility of AZ31 Mg alloy through a torsion extrusion technique, where the torsion-extruded specimen produced texture highly inclined $\left(30^{\circ}\right)$ to the extrusion direction. Huang et al. [151] have reported an increase in the uniform elongation of AZ31 sheet produced by differential speed rolling, where the basal planes were tilted at $15^{\circ}$ to the rolling direction. These findings give hints that mechanical properties can be significantly improved through texture optimization alone.

Basal texture tilt can influence twin formation and their variants can be selected using the Schmid factor (SF) which is a ratio of the resolved shear stress (along the slip or twinning direction) to the axial stress (along the loading direction), and initiates plastic deformation for a given crystal orientation [142]. As an important parameter, the SF is frequently applied to analyze the possibility of the activation of deformation modes with different CRSS values upon certain stress in magnesium [152]. Although the Schmid law is generally used to predict the activated slip system depending on the tensile direction, twinning variants can also be predicted by the highest Schmid factor as reported by many authors [65,118,153-157]. Hong et al. $[118,155]$ and Park et al. [156] reported that different twin variants are active depending on the strain paths (i.e., compression perpendicular to the $c$-axis or tension parallel to the $c$-axis) and their selection mechanism is governed by the Schmid law. By analogy with dislocation activation, a Schmid factor (SF) criterion for twinning variant selection was proposed and validated experimentally for the case of polycrystalline AZ31 [156] and AM30 [65] magnesium alloys, as well as a metastable titanium alloy [154]. Although the calculation of SF and its correlation with the deformation mode in hcp structure is limited, recent studies by Deng et al. 
[158] reported the SF calculation using vector operations to investigate the twinning behavior of Gr2 purity Ti under both quasi-static and dynamic compressive deformation. Nan et al. [159] also calculated Schmid factors for magnesium and other hcp materials [160] as a function of angles between the $c$-axis and the loading direction to interpret deformation behavior. Besides this SF is also possible to calculate using geometric model in a hcp unit cell, where the effect of basal plane tilt angle and loading direction on deformation mode could be incorporated. Therefore, in this study, a model of the hcp crystal structure was used to geometrically calculate the Schmid factor based on the initial basal texture of extruded AM30 alloy. Thus, the purpose of this work is to analytically calculate the influence of basal texture tilt and changes of in-plane loading angle with respect to the extrusion direction on the Schmid factor to select extension twin variants for compressive deformation.

\subsection{Schmid Factor Calculations}

As shown in Fig. 2.2 in Chapter 2 deformation modes in magnesium alloys include the $<\mathbf{a}>$ slip on basal $\{0001\}$, prismatic $\{10 \overline{10}\}$ and pyramidal $\{10 \overline{1} 1\}$ planes, as well as the $\langle\mathbf{c}+\mathbf{a}>$ slip on pyramidal $\{11 \overline{2} 2\}$ planes, and two twinning modes of $\{10 \overline{1} 2\}$ extension twinning and $\{10 \overline{1} 1\}$ contraction twinning [159-161]. The difference of $c / a$ ratio and the use of 4 Miller-Bravais indices for hcp material make the Schmid factor calculation difficult in comparison to fcc materials. However, some useful general equations to calculate the Schmid factor for hcp material are given in [162]. When the load is applied in the direction of $\mathbf{r}_{1}=\left[\begin{array}{lll}u_{l} & v_{l} t_{l} & w_{l}\end{array}\right]$, the slip plane of $\mathbf{p}_{\mathbf{1}}=\left[\begin{array}{llll}h_{1} & k_{1} & i_{1} & l_{1}\end{array}\right]$, slip direction of $\mathbf{r}_{\mathbf{2}}=\left[\begin{array}{lll}u_{2} & v_{2} & t_{2} \\ w_{2}\end{array}\right]$, the Schmid factor (SF) is given by the Eq. 5.1 and 5.2 [162], 


$$
\begin{gathered}
S F=\cos \left(r_{1} \bullet r_{2}\right) \times \cos \left(r_{1} \bullet p 1\right)=\left(\frac{r 1}{\left|r_{1}\right|} \bullet \frac{r_{2}}{\left|r_{2}\right|}\right) \times\left(\frac{r_{1}}{\left|r_{1}\right|} \bullet \frac{p_{1}}{\left|p_{1}\right|}\right), \\
=\frac{u_{1} u_{2}+v_{1} v_{2}+t_{1} t_{2}+\lambda^{2} w_{1} w_{2}}{\left(u_{1}^{2}+v_{1}^{2}+t_{1}^{2}+\lambda^{2} w_{1}^{2}\right)^{1 / 2}\left(u_{2}^{2}+v_{2}^{2}+t_{2}^{2}+\lambda^{2} w_{2}^{2}\right)^{1 / 2}} . \\
\times \frac{u_{1} h_{1}+v_{1} k_{1}+t_{1} i_{1}+w_{1} l_{1}}{\left(u_{1}^{2}+v_{1}^{2}+t_{1}^{2}+\lambda^{2} w_{1}^{2}\right)^{1 / 2}\left(h_{1}^{2}+k_{1}^{2}+i_{1}^{2}+\lambda^{-2} l_{1}^{2}\right)^{1 / 2}} .
\end{gathered}
$$

In a simple way, where $\lambda$ is the angle between the normal of the twinning plane and the loading axis, and $\varphi$ is the angle between their direction of shear and loading axis, Schmid factors, $m$ can be calculated by the following relation [159]:

$$
m=\cos \lambda \cos \varphi
$$

Fig. 5.1(a) shows the two twinning patterns of $\{10 \overline{1} 2\}$ extension twinning and $\{10 \overline{1} 1\}$ contraction twinning, where the schematic relation of the basal plane inclination angle $\beta$, with respect to the extrusion direction is presented in Fig. 5.1(b). Theoretically, extension twinning

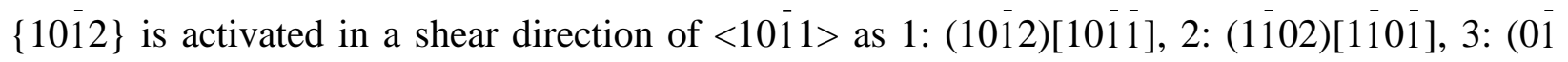

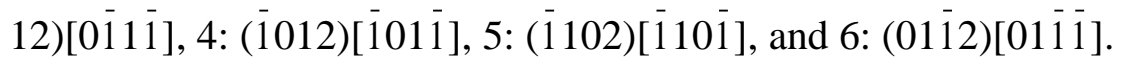

In order to calculate the Schmid factor for the identification of active twin variants with respect to the spatial relationship between the orientation of the lattice and the loading direction, the position of the hcp unit cell of magnesium crystal structure is shown in Fig. 5.2. 

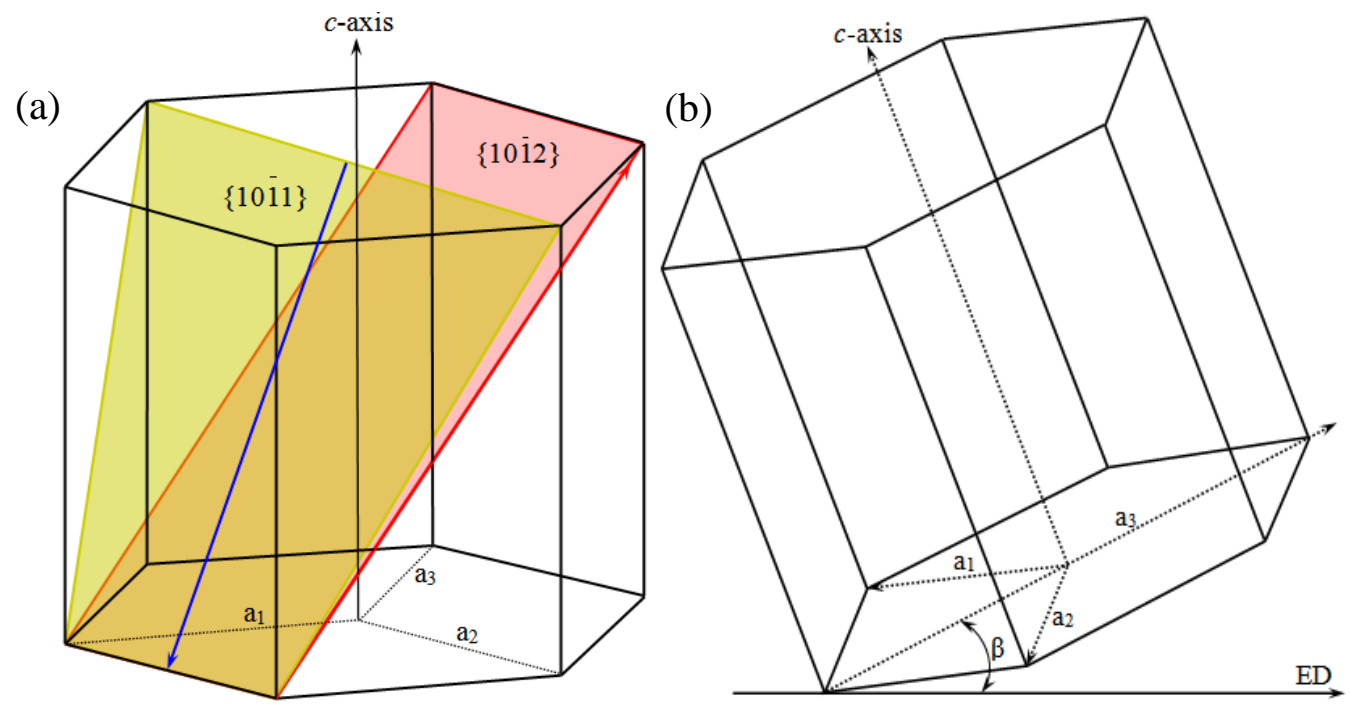

Figure 5.1: (a) Schematic presentation of $\{10 \overline{1} 2\}$ extension and $\{10 \overline{1} 1\}$ contraction twins in magnesium crystal system, (b) considering a basal plane inclination angle $\beta$, with respect to the extrusion direction (ED).

For each variant of twinning as mentioned earlier, the Schmid factor is calculated geometrically by assuming two major concepts: (1) A compressive loading was applied at an in-plane angle $\alpha$ ( $\alpha$ ranges from $0^{\circ}$ to $90^{\circ}$ ) from the extrusion direction, where the $c$-axis of the unit cell would be initially perpendicular to the loading direction, and (2) Basal planes were inclined at an angle of $\beta\left(\beta=0^{\circ}, 15^{\circ}, 30^{\circ}, 45^{\circ}\right)$ from the extrusion direction (Fig. 5.1(b)). An arbitrary (1012)[101̄1] extension twinning variant is shown by a shaded area FEON in Fig. 5.2, where FY is the loading direction, $\mathrm{FN}$ is the twin direction, from $\triangle \mathrm{FYN}$, and $\varphi$ is the angle between the twin direction and the loading direction. From the unit cell (Fig. 5.2), RS intercepts the adjoining line PN at point $S$ and a projection from $S$ to the unit cell intercepts at $\mathrm{S}^{\prime}$, making a triangle $\Delta \mathrm{FSS}^{\prime}$ where $\gamma$ is the angle between $\mathrm{FS}$ and $\mathrm{FS}^{\prime}$. Another triangle $\Delta \mathrm{FS}^{\prime} \mathrm{F}^{\prime}$ is drawn by taking a projection from $\mathrm{S}^{\prime}$ to the sample surface as $\mathrm{F}^{\prime}$ where $(\beta+\gamma)$ is the angle between $\mathrm{FS}$ and $\mathrm{FF}^{\prime}$. 


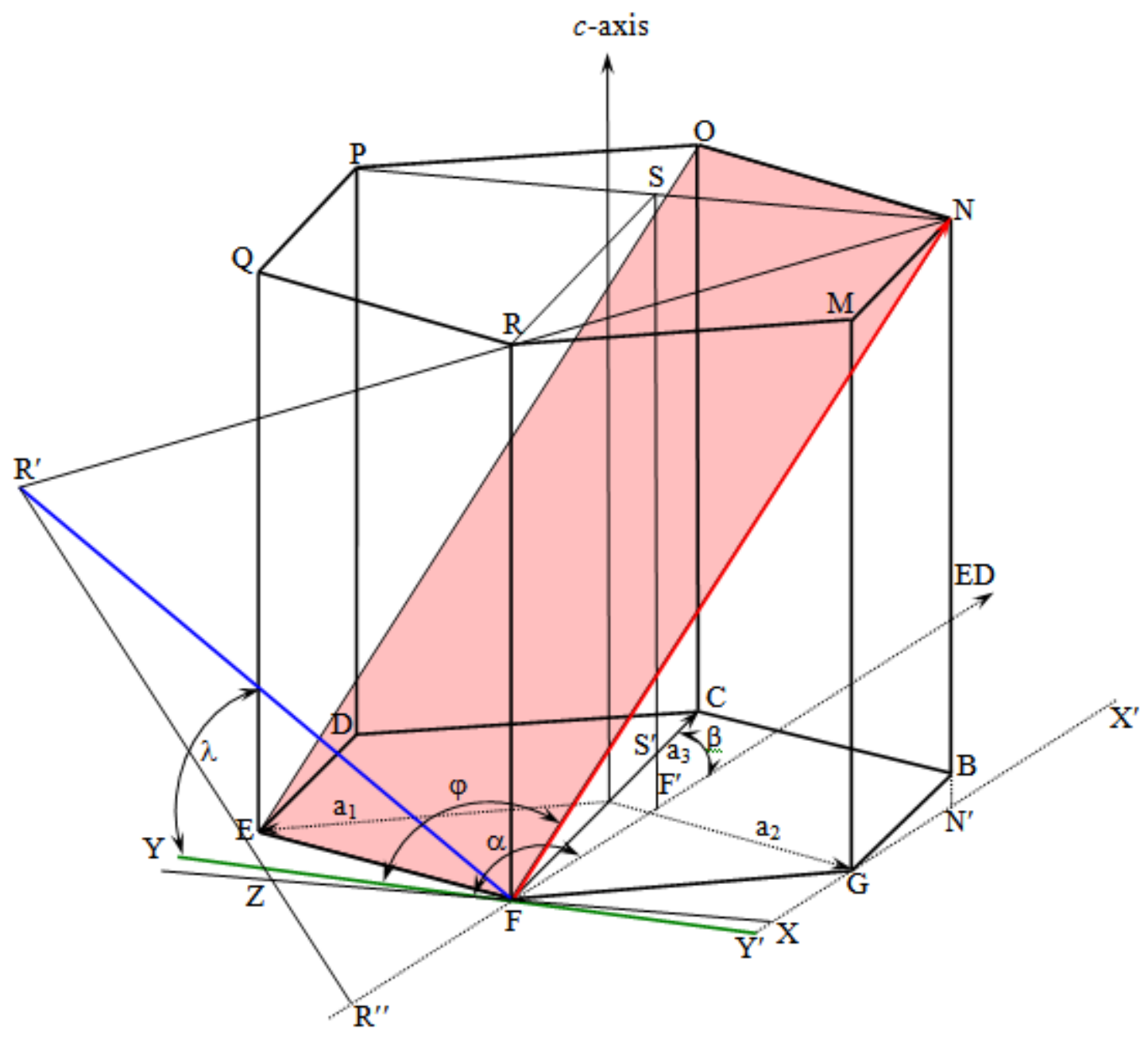

Figure 5.2: Illustration of the hcp crystal structure of magnesium to calculate Schmid factor using the relationship between the angle of compressive loading, twin plane and twin plane normal direction. The shaded FEON is an example of $\{10 \overline{12}\}$ extension twin variant.

The projections from $\mathrm{N}$ to the sample surface is $\mathrm{N}^{\prime}$ and from $\mathrm{Y}$ to extended $\mathrm{XX}^{\prime}$ is $\mathrm{Y}^{\prime}$. $\mathrm{FR}^{\prime}$ is the twin normal on FN, which intercepts the extended $N R$ at point $R^{\prime}$, a projection from $R^{\prime}$ to the sample surface intercepts at point $\mathrm{R}^{\prime \prime}$ (Fig. 5.2). A perpendicular line to the extrusion direction (ED), passes through the point $\mathrm{R}^{\prime}$, intercepts the extended $\mathrm{OR}$ at point $\mathrm{O}^{\prime}$ and makes a right triangle $\Delta \mathrm{RR}^{\prime} \mathrm{O}^{\prime}$ (Appendix A). A projection from $\mathrm{O}^{\prime}$ to the sample surface intercepts at point $\mathrm{O}^{\prime \prime}$ 
(Fig. A1) and $\mathrm{R}^{\prime} \mathrm{R}^{\prime \prime}=\mathrm{O}^{\prime} \mathrm{O}^{\prime \prime}$. The angle between the loading direction FY and the twin direction $\mathrm{FN}$ is $\varphi$, which can be calculated from the $\triangle \mathrm{FYN}$ using cosine rule:

$$
\begin{gathered}
\cos \varphi=\frac{(F Y)^{2}+(F N)^{2}-(Y N)^{2}}{2(F Y)(F N)}, \\
\cos \varphi=\frac{2 \cdot \sqrt{\mathrm{c}^{2}+(9 / 4) \mathrm{a}^{2}} \cdot \cos \left(\beta+\sin ^{-1}\left(\frac{\mathrm{c}}{\sqrt{\mathrm{c}^{2}+(9 / 4) \mathrm{a}^{2}}}\right)\right) \cdot \sin \alpha-\mathrm{a} \sqrt{3} \cdot \cos \alpha}{2 \cdot \sqrt{3 \mathrm{a}^{2}+\mathrm{c}^{2}}},
\end{gathered}
$$

$F^{\prime}$ is the twin normal, which intercepts the extended $N R$ at point $\mathrm{R}^{\prime}$. Considering the $\Delta F Y R^{\prime}$, the angle between FY and FR' which is $\lambda$, can be calculated using cosine rule as:

$$
\begin{gathered}
\cos \lambda=\frac{(F Y)^{2}+(F R)^{2}-\left(R^{\prime} Y\right)^{2}}{2(F Y)\left(F R^{\prime}\right)} \\
\cos \lambda=\frac{\sqrt{\left(4+3 \tan ^{2} \theta\right) \cdot \sin ^{2}(\beta+\delta)+\tan ^{2} \theta \cdot \cos (\alpha+\eta) \cdot \cos \theta}}{2}
\end{gathered}
$$

\subsection{Influence of Loading Direction and Basal Plane Inclination on Schmid Factor}

The SF values of the $\{10 \overline{1} 2\}$ twin variants were calculated and plotted as a function of the angle of loading direction from the extrusion direction, $\alpha$, for different basal plane inclination angles of $\beta$, as shown in Fig. 5.3. Based on the Schmid law, for a specific position the most favorably activated variant should be the one possessing the maximum SF. 
The basal plane inclination, together with in-plane loading direction with respect to the extrusion direction, has a significant effect on the SF value. When there is no inclination of the basal plane $(\beta=0)$, the loading along the extrusion direction $(\alpha=0)$ gave a SF value of 0.374 for four twin

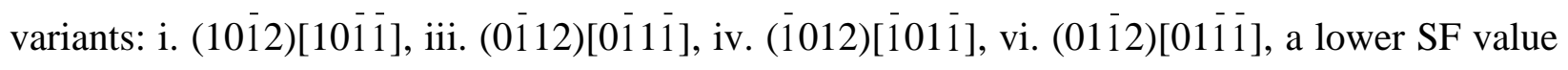

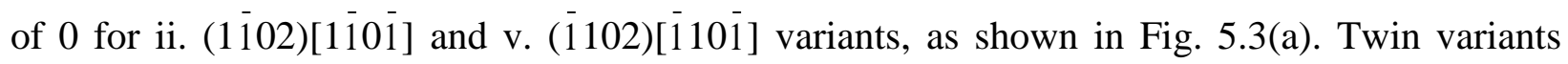
(1102)[1101] and (1102)[1 101] showed a maximum SF value of 0.498 at $\alpha=90^{\circ}$. The SF value

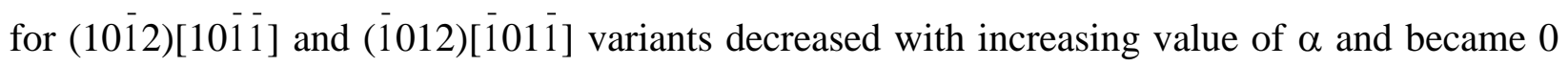

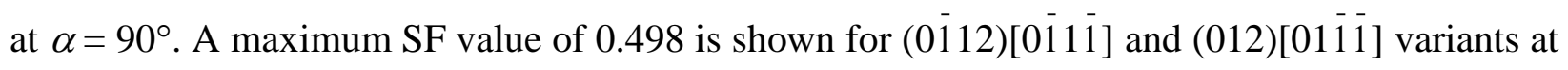
$\alpha \sim 30^{\circ}$, and then decreased to 0 at $\alpha=90^{\circ}$. A decreasing trend of SF values for all variants is observed in Fig. 5.3(b), at a small inclination angle of $\beta=15^{\circ}$ with initial loading direction of $\alpha$ $=0^{\circ}$.

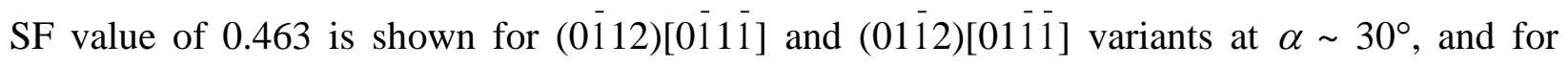
(1102)[1ํㅣ] and (1102)[110i] variants maximum $\mathrm{SF}$ value was 0.498 at $\alpha=90^{\circ}$. With increasing inclination angle to $\beta=30^{\circ}$, as shown in Fig. 5.3(c), all variants showed the lowest SF values, whereas (1102)[1ㅣㅣ] and (1102)[110i] variants gave the same maximum SF value of 0.498 at $\alpha=90^{\circ}$. It is interesting to note that at $\beta=45^{\circ}$, in Fig. 5.3(d), the lowest value of SF is

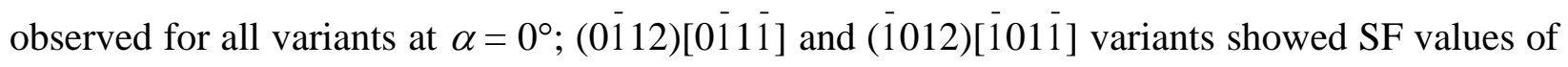

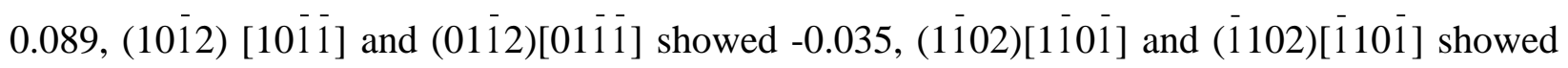
-0.249 . 

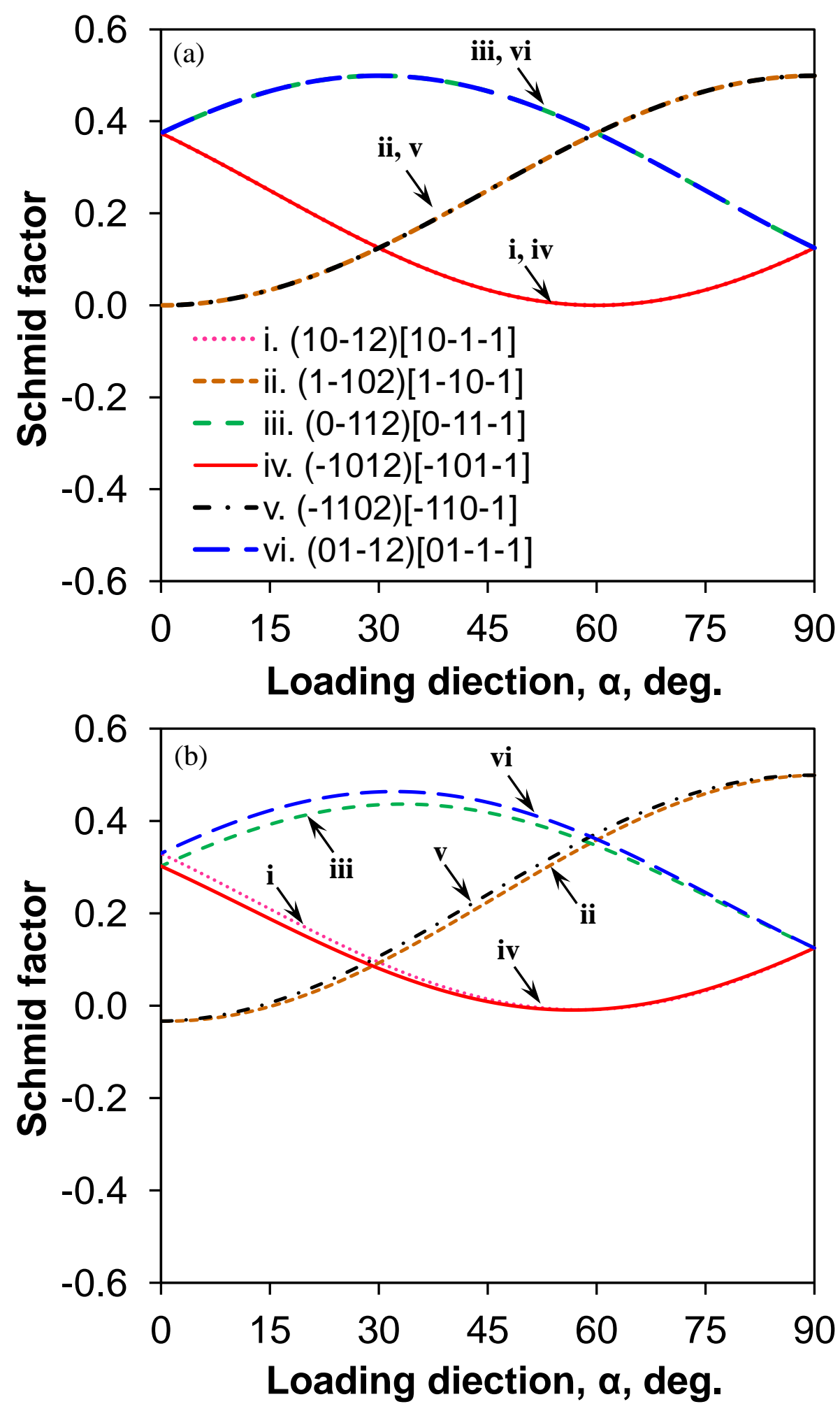

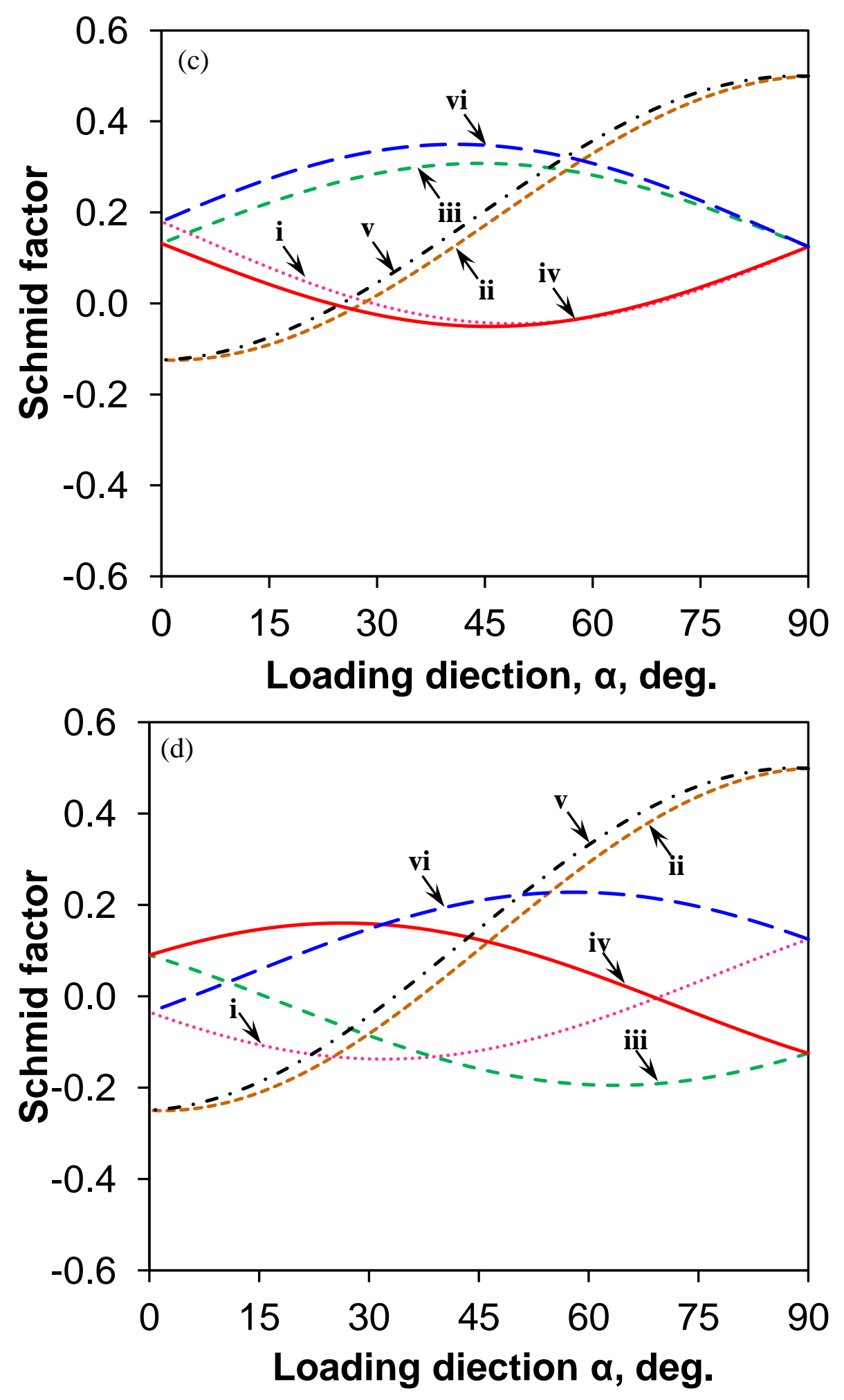

Figure 5.3: Schmid factors of $\{10 \overline{1} 2\}$ extension twins as a function of loading axis with the extrusion direction at different $\beta$ angles of (a) $0^{\circ}$, (b) $15^{\circ}$, (c) $30^{\circ}$, and (d) $45^{\circ}$. 


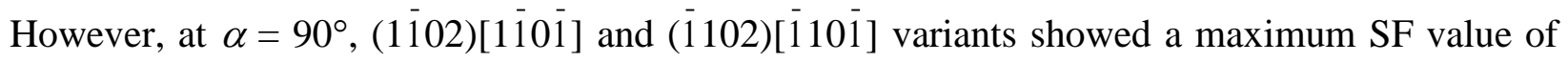
0.498 .

The results of the calculations of the SF values for extension twin variants are further plotted as a function of both angles $\alpha$ and $\beta$, which is shown in Fig. 5.4. Taking into account the symmetry of hcp, there will be six twin variants for the extension twins in which three are independent [163].

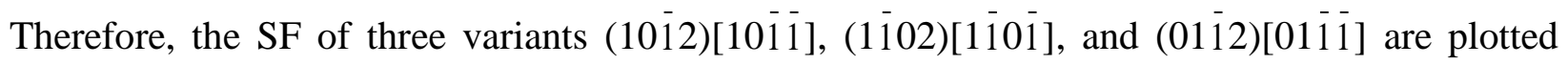
with respect to $\beta$ and $\alpha$. Twin variant (1012)[101]] showed a SF value $\sim 0.3$, in a range of $\alpha=0^{\circ}$ to $20^{\circ}$ and $\beta=0^{\circ}$ to $25^{\circ}$. A lower SF value is observed for the (1102)[1] 101$]$ variant, at initial position of $\alpha=0$ and $\beta=0$, which increased to $>0.4$ with increasing the angle $\alpha$, from $60^{\circ}$ to $90^{\circ}$, and showed a maximum SF value of 0.498 at $\alpha=90^{\circ}$ and at this range, the SF value is independent of $\beta$. A wide range of higher $\mathrm{SF}$ value of $>0.4$ is observed for $(01 \overline{1} 2)[01 \overline{1} \overline{1}]$ variants for $\alpha=0^{\circ}$ to $60^{\circ}$ and $\beta=0^{\circ}$ to $15^{\circ}$.

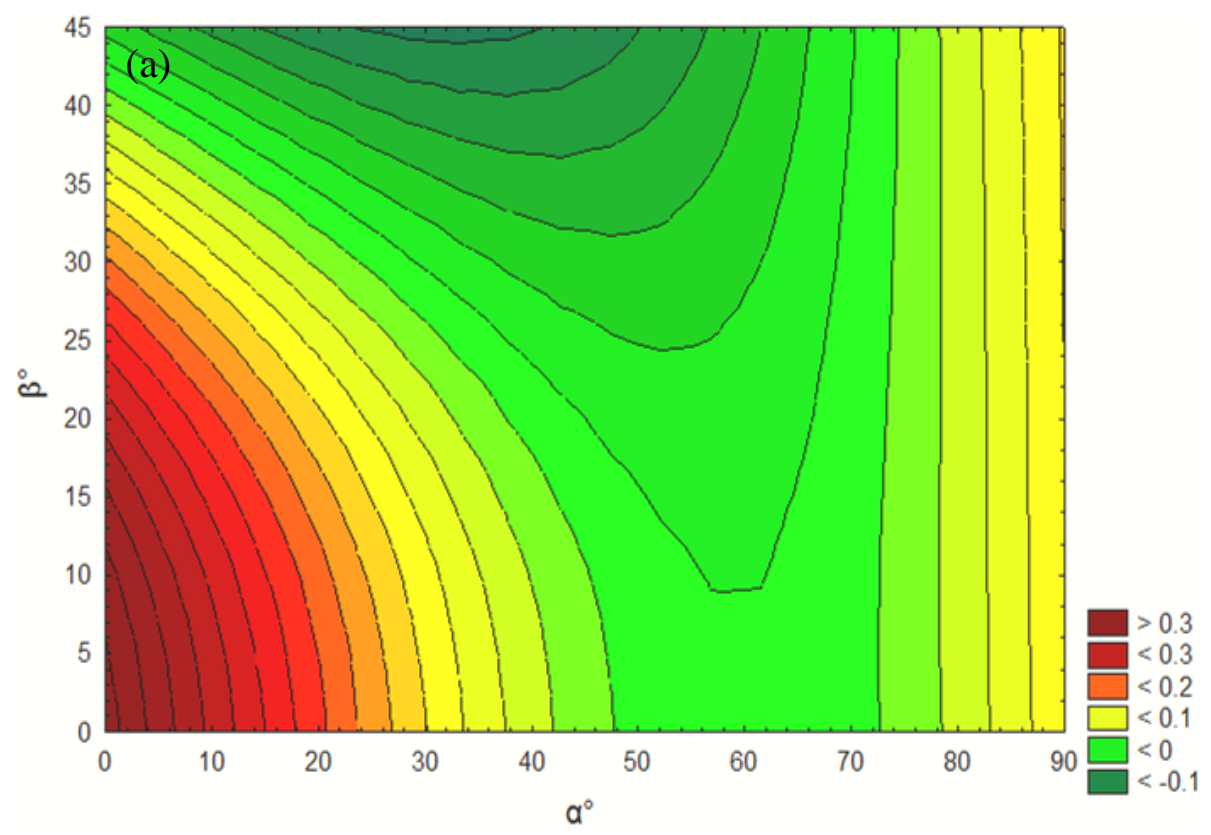



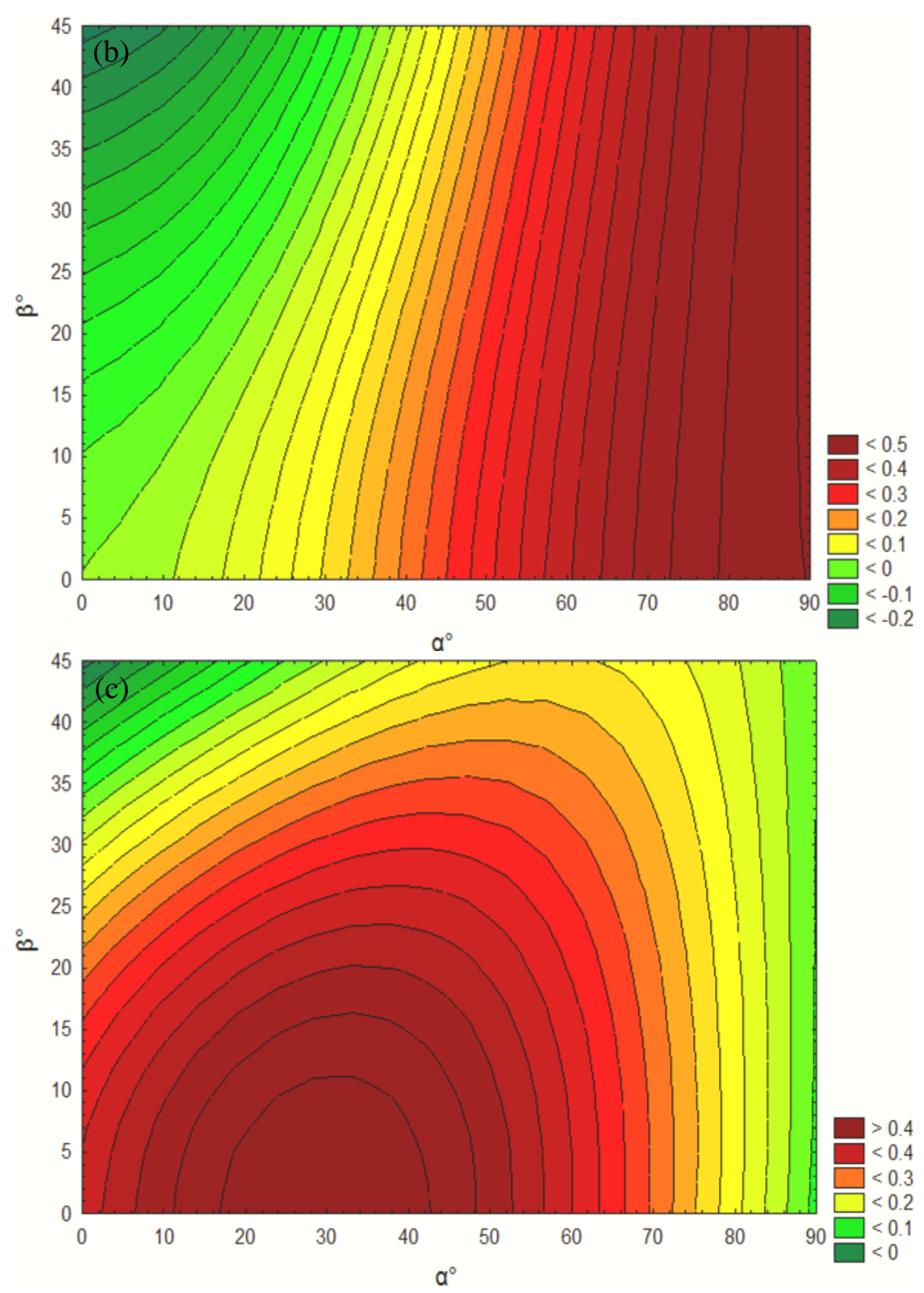

Figure 5.4: Schmid factors as a function of loading axis $(\alpha)$ and basal plane inclination angle $(\beta)$

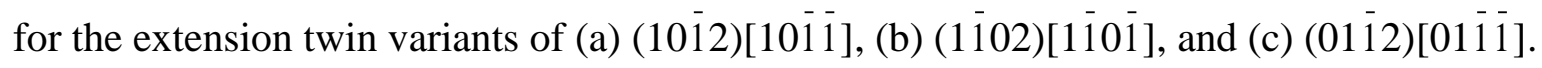

Using a well established equation from [162], and HEXASchmid software, SF values were calculated and shown in Table 5.1, considering a $0^{\circ}$ inclination angle and compression along the 
extrusion direction. This comparison indicates that four calculated variants have the same SF value of 0.374 as observed from Eq. 5.2 [162] and HexaSchmid program while two variants show a SF value of 0 . Many authors also indicated a discrepancy between the actual and calculated SF values and explained it as elastic and plastic anisotropy resulting from texture [142].

Table 5.1: A comparison of SF values for all the extension twin variants using different methods at a $0^{\circ}$ inclination angle and compression along the extrusion direction.

\begin{tabular}{|c|c|c|c|}
\hline $\begin{array}{c}\text { Extension } \\
\text { twin variants }\end{array}$ & $\begin{array}{c}\text { SF value using } \\
{[27]}\end{array}$ & $\begin{array}{c}\text { SF value using } \\
\text { HexaSchmid } \\
\text { software }\end{array}$ & $\begin{array}{l}\text { SF value in the } \\
\text { present study }\end{array}$ \\
\hline$(1012)[1011]$ & 0.374 & 0.374 & 0.374 \\
\hline$(1 \overline{1} 02)[1 \overline{1} 0 \overline{1}]$ & 0 & 0 & 0 \\
\hline$(0 \overline{1} 12)[0 \overline{1} 1 \overline{1}]$ & 0.374 & 0.374 & 0.374 \\
\hline (1012) [1011] & 0.374 & 0.374 & 0.374 \\
\hline (1102) [110̄1] & 0 & 0 & 0 \\
\hline$(01 \overline{1} 2)[01 \overline{1} \overline{1}]$ & 0.374 & 0.374 & 0.374 \\
\hline
\end{tabular}

The results above can be used to analyze the effect of texture on deformation behavior [38,41,69,97,164-166]. In an extruded AM30 alloy, containing an initial texture (Fig. 4.1(b)), where $\beta=0^{\circ}$ to $20^{\circ}$ and $\alpha=0^{\circ}$, four extension twin variants $(10 \overline{1} 2)[10 \overline{1} \overline{1}],(0 \overline{1} 12)[0 \overline{1} 1 \overline{1}],(\overline{1} 012)$

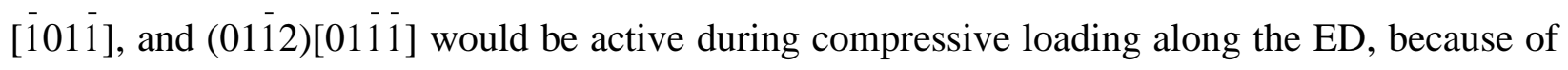
their higher SF value in that range (Fig. 5.3(a)). Their formation is evident from texture development (Fig. 4.7(b)) after compression along the extrusion direction, as the occurrence of $\{10 \overline{1} 2\}$ extension twinning re-orients the crystallographic lattice by $86.3^{\circ}[19,49,129,167]$. 
Another two (1102)[1시] and (1102)[110i] variants would be active with increasing loading angle from the extrusion direction. Basal plane inclination showed a significant effect on the SF value as four variants showed a decreasing value with increasing $\beta$, except the (1102)[110i] and (1102)[1101] variants, as they are independent of $\beta$. Thus active twin variants reflected from the highest Schmid factor value were significantly dependent on the activation mode (i.e., tension parallel or compression perpendicular to the $c$-axis) which was governed by the combination of the crystallographic lattice orientation and the applied loading direction.

\subsection{Effect of In-plane Loading Direction on Compressive Behavior}

Cylindrical samples $(\Phi 5 \mathrm{~mm} \times 8 \mathrm{~mm})$ were taken with the cylinder (compression) axis oriented at $0^{\circ}, 15^{\circ}, 30^{\circ}, 45^{\circ}$ and $90^{\circ}$ to the extrusion direction (ED) (Fig. 3.2). The flow stress behavior of the samples during compression along $0^{\circ}, 15^{\circ}, 30^{\circ}, 45^{\circ}$ and $90^{\circ}$ to the ED are shown in Fig. 5.5. From the true stress-strain curves (Fig. 5.5(a)), the yield strength (YS), and ultimate compressive strength (UCS) are plotted in Fig. 5.5(b), as functions of sample orientation (or compressive axis angle) with respect to the ED. The results from the compression tests showed almost constant YS of $\sim 90 \mathrm{MPa}$ and UCS of $380 \mathrm{MPa}$ at different sample orientations with a remarkable compressive hardening capacity of about 3.2, where the hardening capacity index was defined as (UCS-YS)/YS [168]. The fracture strain showed an increasing trend with increasing in-plane orientation angle up to $45^{\circ}$, and then decreased at $90^{\circ}$ with respect to the ED (Fig. 5.5(c)). These variations of compressive properties are directly related to the twin formation and texture change during plastic deformation [135,169], which will be discussed later. 

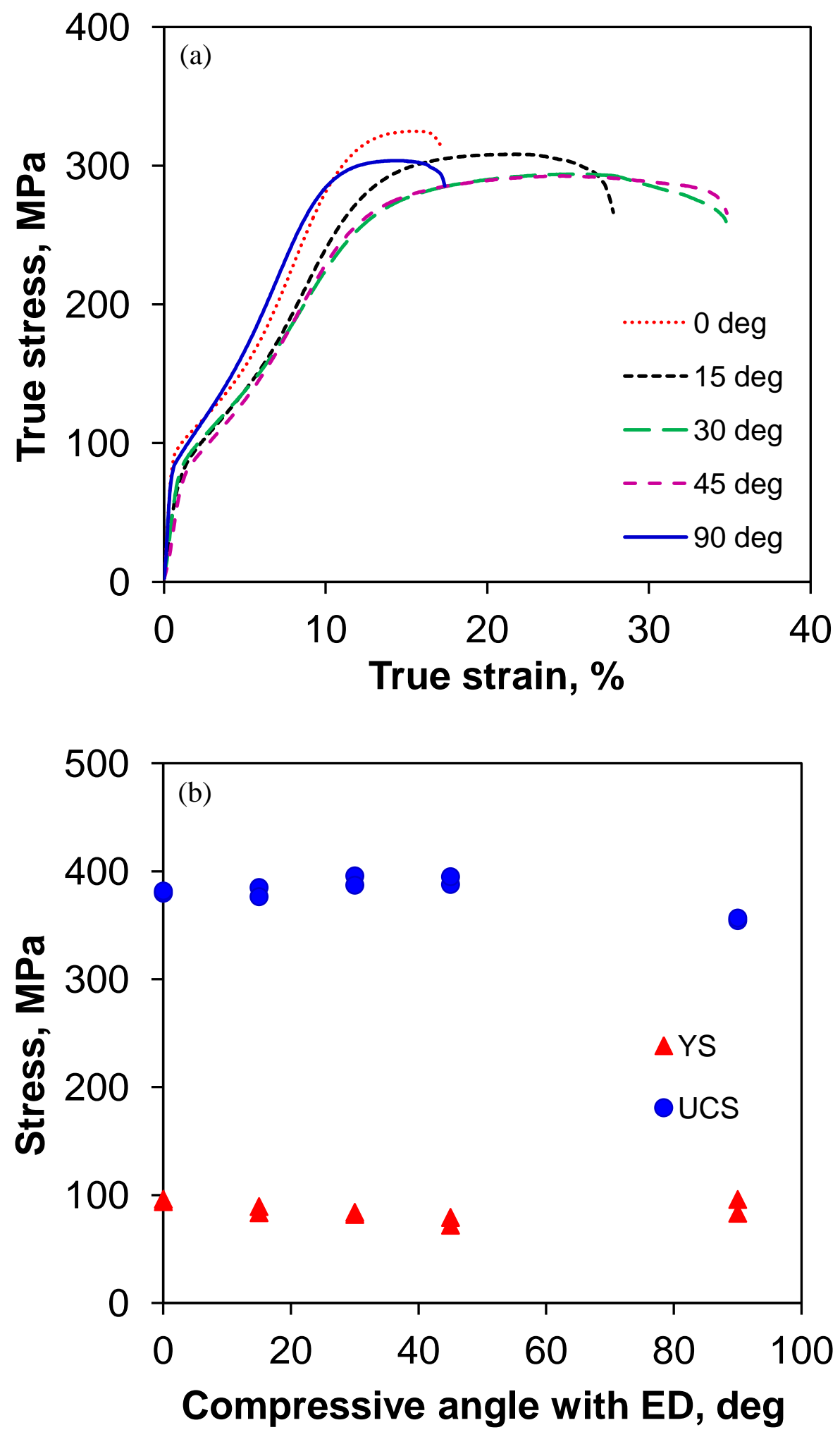

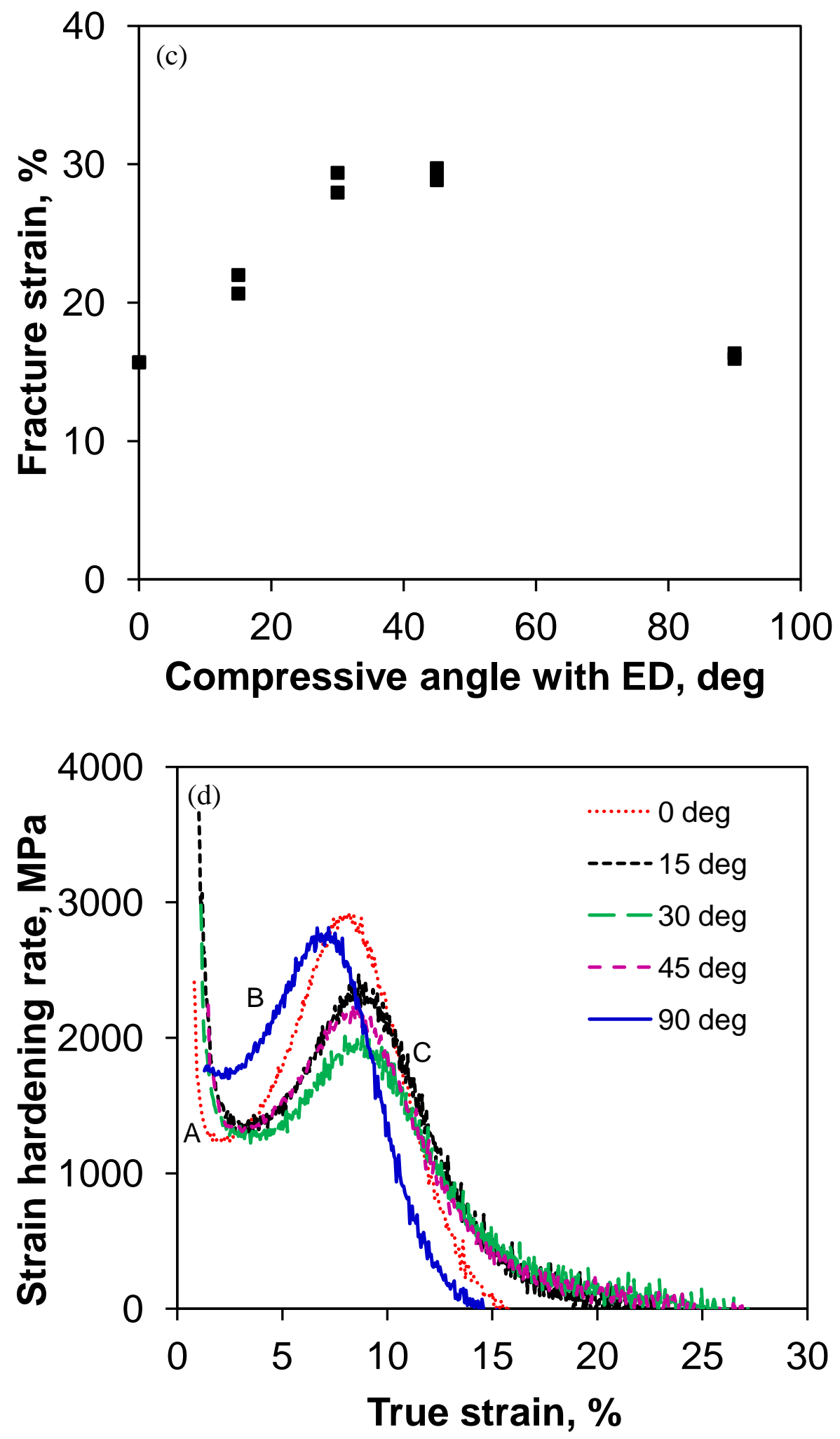


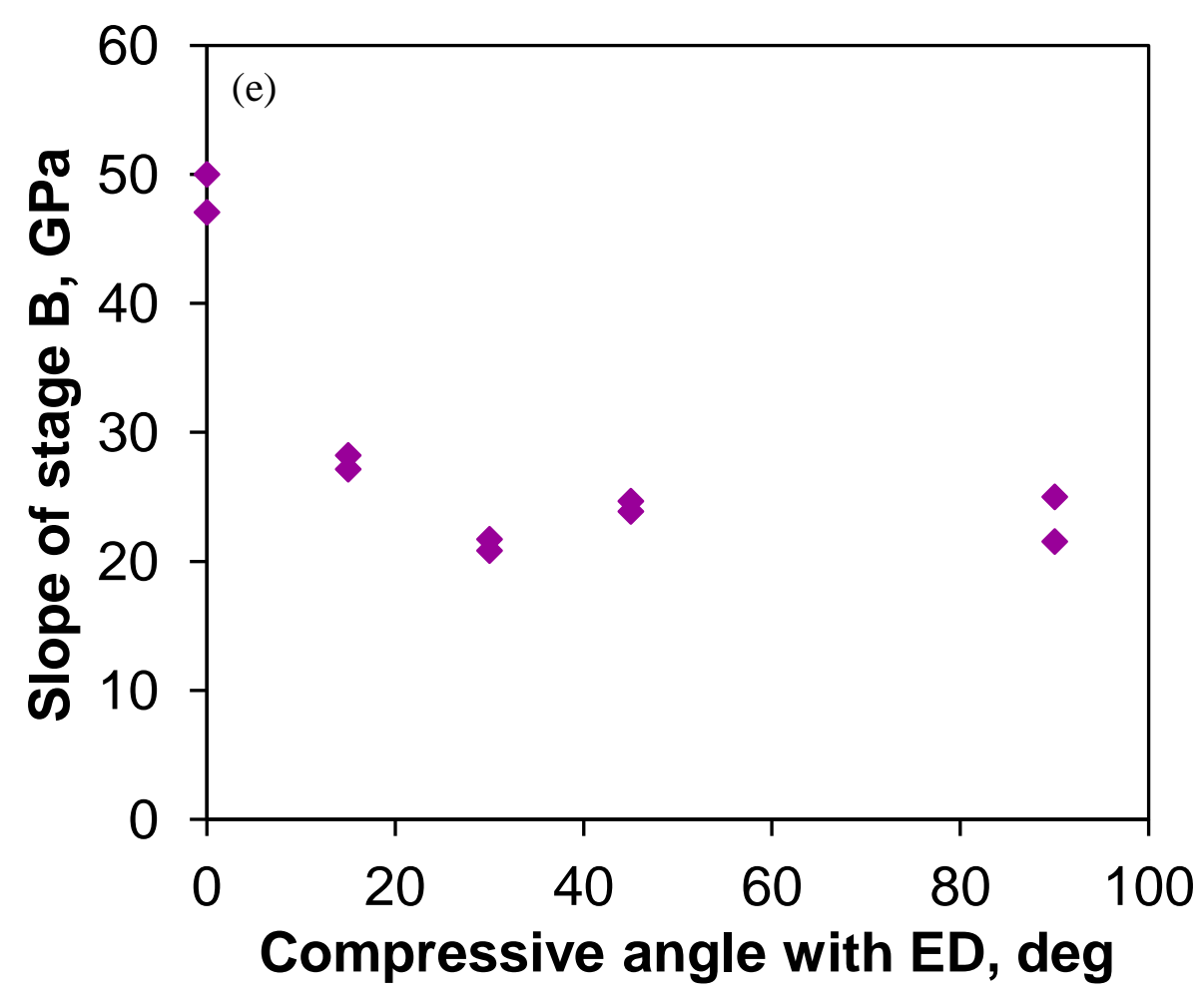

Figure 5.5: (a) Compressive true stress-true strain curves, (b) yield strength (YS) and ultimate compressive strength (UCS) and (c) fracture strain as a function of sample orientation, (d) strain hardening rate as a function of true strain, and (e) the slope of stage B in the strain hardening rate vs. sample orientation in the extruded AM30 magnesium alloy.

To better view the flow stress behavior, the strain hardening rate $d \sigma / d \varepsilon$ (where $\sigma$ is true stress) with respect to true strain $\varepsilon$ is plotted in Fig. 5.5(d). From these curves three stages of strain hardening (defined as A, B and C in [135]) could be clearly distinguished for all samples oriented differently. Stage A was characterized by a rapidly decreasing strain hardening rate up to a compressive strain of approximately $2.5 \sim 3.5 \%$, followed by stage B with an increasing strain hardening rate up to a compressive strain of 7 9\%, and then stage $\mathrm{C}$ with an decreasing 
strain hardening rate again until failure. Similar trends in the strain hardening rate have also been observed in $\alpha$-titanium [127], AM30 and AZ31 magnesium alloys [128,135,170].

A decreasing slope of stage B in strain hardening rate with increasing compressive axis angle from $0^{\circ}$ to $15^{\circ}$ to $30^{\circ}$ with respect to the ED was observed (Fig. 5.5(e)). This suggested that the strain hardening rate also depended on the in-plane loading direction. A slight increase in the slope for the $45^{\circ}$ and $90^{\circ}$ samples compared to the $30^{\circ}$ sample occurred as well.

To understand such a flow stress behavior, the microstructure development of the samples oriented at $0^{\circ}, 45^{\circ}$ and $90^{\circ}$ from the $\mathrm{ED}$ and compressed at a strain of $4.3 \%$ was examined.

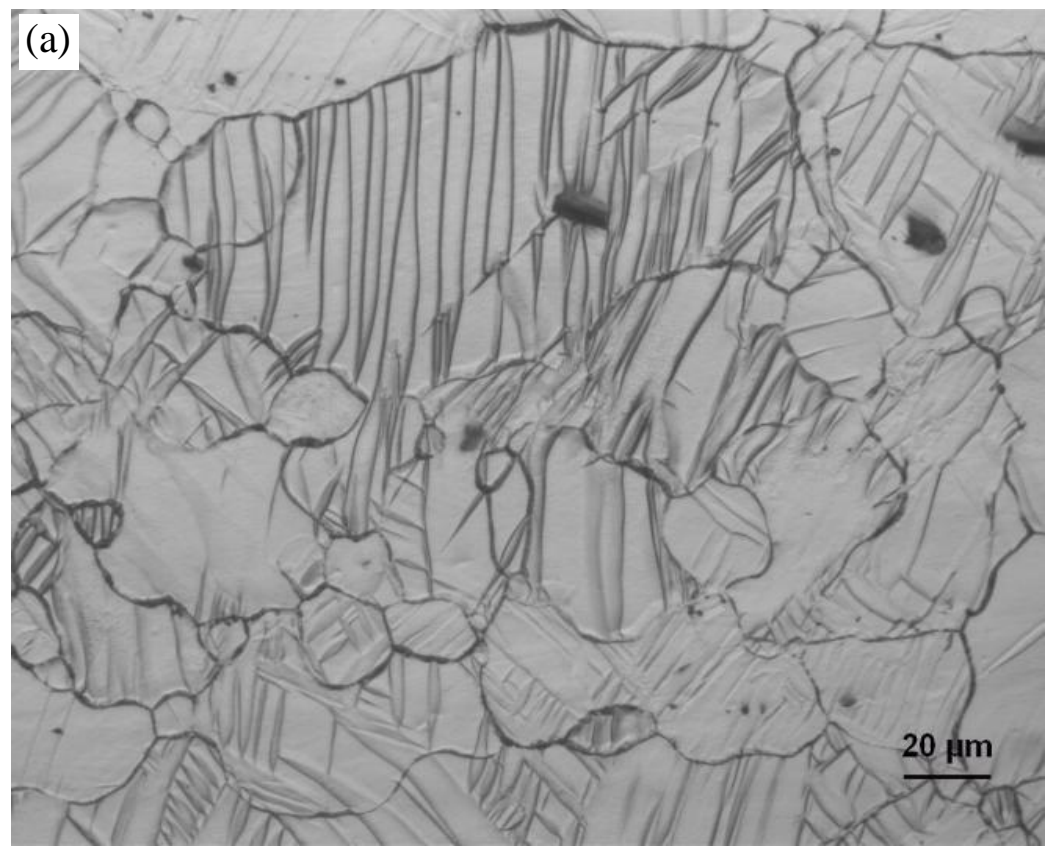



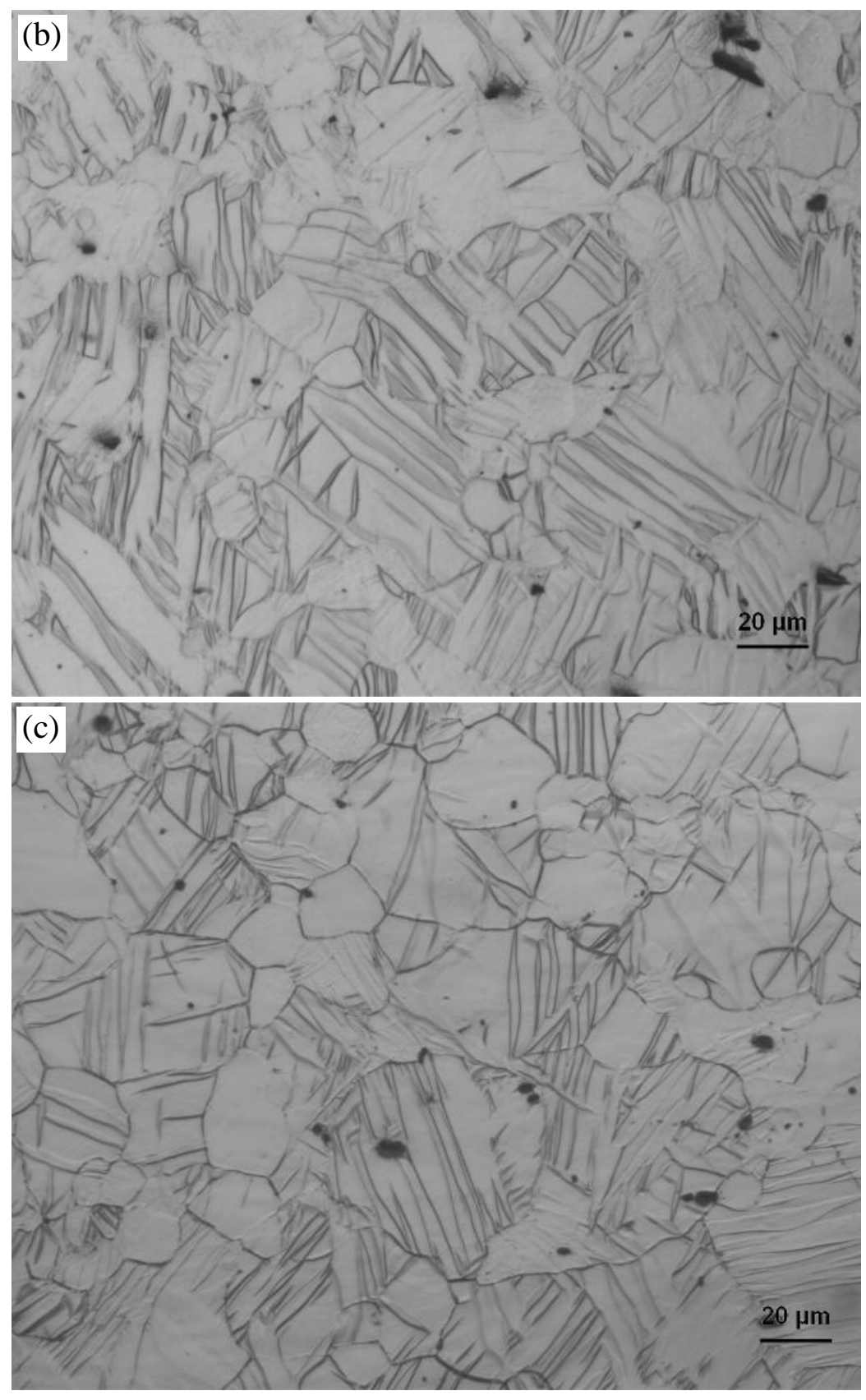

Figure 5.6: Typical microstructure observed at a strain level of $4.3 \%$ in samples oriented at an angle of (a) $0^{\circ}$, (b) $45^{\circ}$ and (c) $90^{\circ}$ relative to the ED.

Some typical images are shown in Fig. 5.6, where the compressive axis was parallel to the horizontal direction. At the strain level of $4.3 \%$, all three samples lay within stage B of strain 
hardening (Fig. 5.5(d)), and the presence of twins can be clearly seen in all the samples. This observation was consistent with the previous studies in $[116,135,169]$, where in the extruded magnesium alloys extension twins were reported to occur in the strain range of $2-4 \%$. The microstructural observations indicated that the twin formation was directly related to the sample orientation. In all three differently oriented samples, the $c$-axis was perpendicular to the compressive axis and thus favored twin formation. Early experiments on Mg single crystals [35] showed that compression perpendicular to the $c$-axis led to the occurrence of extension twinning when the $c$-axis was not constrained. It has been reported that in the extruded material $c$-axis was more concentrated perpendicular to the ED than to the TD [35,170], which caused a higher extent of twinning along the ED than along the TD, which was also evident in the current extruded AM30 alloy in Fig. 5.6(a) and (c).

This could be confirmed from the texture results shown in Fig. 5.7 in terms of pole figures measured from the samples oriented at an angle of $0^{\circ}$ (Fig. $4.7(\mathrm{~b})$ ), $45^{\circ}$ and $90^{\circ}$ from the ED and compressed at a strain of $4.3 \%$. Compared with the initial textures (Fig.4.1(b)), it is clear that twinning gave rise to a marked orientation change for all three samples with increasing compressive strain from 0 to $4.3 \%$. As already described in Fig. 4.7(b), compression along the ED $\left(0^{\circ}\right.$ oriented sample) at a strain level of $4.3 \%$, (0001) the basal poles showed maximum intensity of 7.7 MRD towards the ED and prismatic (1010) poles towards the center. In the $45^{\circ}$ oriented sample (Fig. 5.7(a)), basal (0001) poles were rotated to a location at $45^{\circ}$ from the ED with a maximum intensity of 6.5 MRD. On the other hand, more intense (0001) poles rotated towards the TD and (1010) poles along the ED were observed after compression of the $90^{\circ}$ oriented sample (Fig. 5.7(b)). 


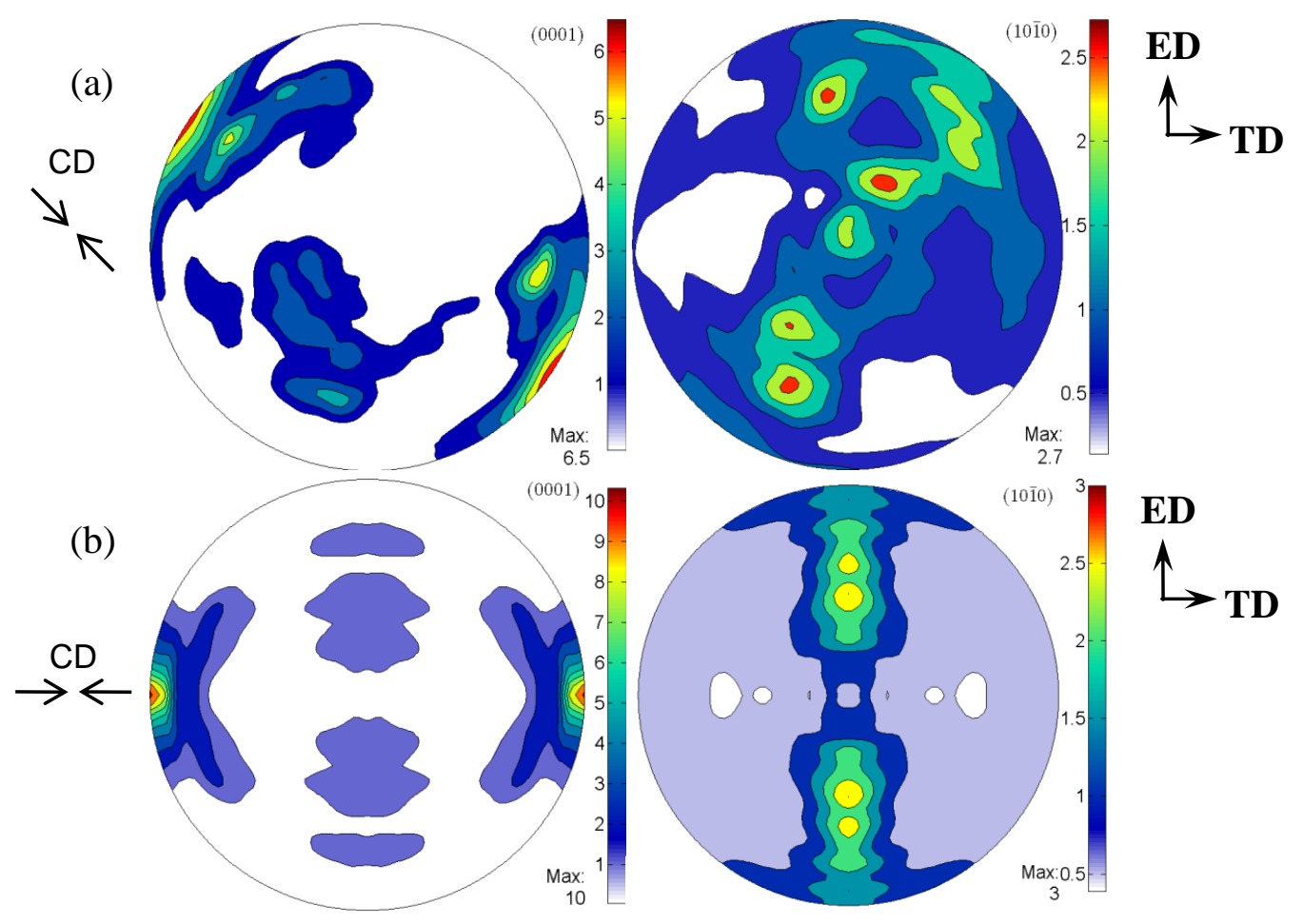

Figure 5.7: Pole figures obtained from the samples oriented at an angle of (a) $45^{\circ}$ and (b) $90^{\circ}$ from the ED and compressed at a strain amount of $4.3 \%$, where $\mathrm{CD}$ stands for the compression direction.

The texture evolution was related to the twin formation with increasing compressive strain level [135,169-175]. As shown earlier in Fig. 5.6, a lot of twins formed after compressive deformation at a strain of $4.3 \%$. The occurrence of extension twinning during compression caused the $c$-axes of most grains to rotate by $\sim 86.3^{\circ}$, always towards the compression direction [169]. This is clearly perceived in the pole figures shown in Fig. 5.7, where the (0001) poles were always rotated against the compressive direction (CD) (Fig. 4.7(b), 5.7(a)-(b)). That is, in the $0^{\circ}$ and $90^{\circ}$ oriented samples after compression to $4.3 \%$ strain, the $c$-axes in almost all grains became parallel to the ED and TD, respectively, orientations which were un-favorable for further twinning and 
slip and thus became more strain hardened (Fig. 5.5(d)). This was corroborated by the lower fracture strain in these two cases (Fig. 5.5(c)).

The calculated SF values at different crystallographic and loading orientations can be used to explain the changes in compressive properties at different in-plane loading directions. With the initial texture of extruded AM30 alloy where the $c$-axis was at a $20^{\circ}$ tilt to $\operatorname{ED}\left(\beta=20^{\circ}\right)$, compression along the $0^{\circ} \mathrm{ED}\left(\alpha=0^{\circ}\right)$, showed a SF value of 0.374 for four extension twin variants, indicating higher possibility of twinning activity as evident from the microstructure (Fig. 5.6) and the skewed shape in the stress-strain (Fig. 5.5(a)) curve with lower fracture strain (Fig. 5.5(c)). With increasing in-plane loading angle to $15^{\circ}, 30^{\circ}$, and $45^{\circ}$ to the ED, two twin variants showed a maximum SF value of 0.498 . Moreover, in the $45^{\circ}$ oriented samples, almost all $c$-axes were positioned at $45^{\circ}$ to the ED due to the formation of extension twins, and further straining could continue the twinning or slip process which would be the reason for the higher fracture strain (Fig. 5.5(c)).

\subsection{Summary}

The presence of crystallographic texture in wrought magnesium alloys causes mechanical anisotropy and tension-compression yield asymmetry. The basal texture tilt can influence the formation of twins, and their variants can be identified using the Schmid factor (SF). In this chapter, a geometrical model of hexagonal close packed (hcp) structure was proposed to calculate the Schmid factor value. The dependence of the Schmid factor on the crystallographic orientations and compressive loading directions was obtained, thus the active twin variants 
during deformation could be identified. To verify the proposed model for calculating the SF value, compressive deformation was also conducted using cylindrical samples of different orientations with respect to the extrusion direction.

The basal plane inclination and loading direction have a significant effect on the SF, which is calculated based on a geometrical model proposed. When there is no basal plane inclination, compressive loading along the ED showed four extension twin variants with a high SF value, indicating a high probability of twin formation. With increasing inclination angle and the inplane loading direction with respect to the ED, only two variants showed such a high SF value. The results suggest that the active twin variants reflected by the high Schmid factor value were significantly dependent on the activation mode (i.e., tension parallel or compression perpendicular to the $c$-axis) which was governed by the combination of the crystallographic lattice orientation with the applied loading direction.

The in-plane loading direction with respect to the ED has a significant impact on compressive properties. At different compressive loading directions, from $0^{\circ} \mathrm{ED}$ to $90^{\circ} \mathrm{ED}$, nearly constant compressive yield strength of $\sim 90 \mathrm{MPa}$ and ultimate compressive strength of $\sim 380 \mathrm{MPa}$ were obtained with a notable hardening capacity index of $\sim 3.2$. However, the fracture strain first increased with increasing loading angle up to $45^{\circ} \mathrm{ED}$ and then decreased at $90^{\circ} \mathrm{ED}$. The slope of the strain hardening rate curve in stage B showed a decreasing trend with increasing compressive axis angle from the ED. 


\section{CHAPTER 6}

\section{Role of Pre-compressive Deformation on Twinning, Texture and Hardening Behavior}

\subsection{Introduction}

The activation of $\{10 \overline{1} 2\}$ extension twinning in the extruded magnesium alloy causes the $c$-axes in the twinned regions to be aligned towards the compression direction from the basal (0001) texture where the $c$-axes in most grains are initially positioned perpendicularly to the loading axis [129], as shown in Fig. 4.1. It has also been reported that the twinned areas can be aligned to either twin growth or de-twinning if the strain path (or loading path) is changed appropriately $[3,34,67,69,81-85]$, which can have a significant influence on the behavior of magnesium alloys during forming, e.g., rolling, leveling, coiling, bending, stretching, etc. since it can result in an increase or decrease in the flow stress when such alloys are subjected to strain reversals $[11,173]$. The microstructure evolution that influences hardening and texture behavior upon re-loading (both longitudinal, transverse and normal) depends on the pre-strain amounts [11]. However, it is unclear how twinning and de-twinning occur with a change of strain path, if the formation of new twins and de-twinning can be co-existent, and how they affect the texture change and hardening behavior. It is therefore necessary to understand the effect of reversing the strain path on the stress-strain response, as well as the role of twinning in bringing this about. The objective of this study was, therefore, to identify the influence of twins introduced by pre-straining on the subsequent texture and hardening behavior of an extruded AM30 alloy upon re-loading with and without changing the strain path. 


\subsection{Effect of Loading Direction on Compressive Properties}

The true stress-true strain curves of the initially as-extruded AM30 magnesium alloy compressed in the ED, TD and ND directions are shown in Fig. 6.1. It is seen that both the ED and TD curves were similar and the average compressive yield stress was $~ 91 \mathrm{MPa}$ in both the ED and TD directions. Also, both curves showed a skewed/sigmoidal shape, indicative of the activation of deformation twinning $[98,129,135]$. An ultimate compressive strength of $\sim 325 \mathrm{MPa}$ and $\sim 295$ MPa was observed along the ED and TD, respectively. However, compression through the ND showed a true stress-true strain curve that appeared similar to a slip dominated one (e.g., for fcc and/or bcc metals), with a higher yield stress of $\sim 175 \mathrm{MPa}$ and compressive strength of $\sim 330$ $\mathrm{MPa}$. When compressed in the ED, the true stress increased more rapidly with increasing true strain, compared to the TD and ND. Because of the presence of strong basal textures in the asextruded material with the $c$-axes of most grains aligned almost parallel to the ND (Fig. 4.1(b) and (c)), for both the ED and TD samples, compressive loading was perpendicular to the $c$-axes, whereas for the ND sample, it was parallel to the $c$-axis. Such a loading condition facilitated the occurrence of the $\{10 \overline{1} 2\}$ extension twinning in both the ED and TD. Therefore, the flow curves of ED and TD exhibited a similar sigmoidal shape (Fig. 6.1), which is known to be a typical feature of twinning-dominated deformation $[11,20,21,112,129,135]$. 


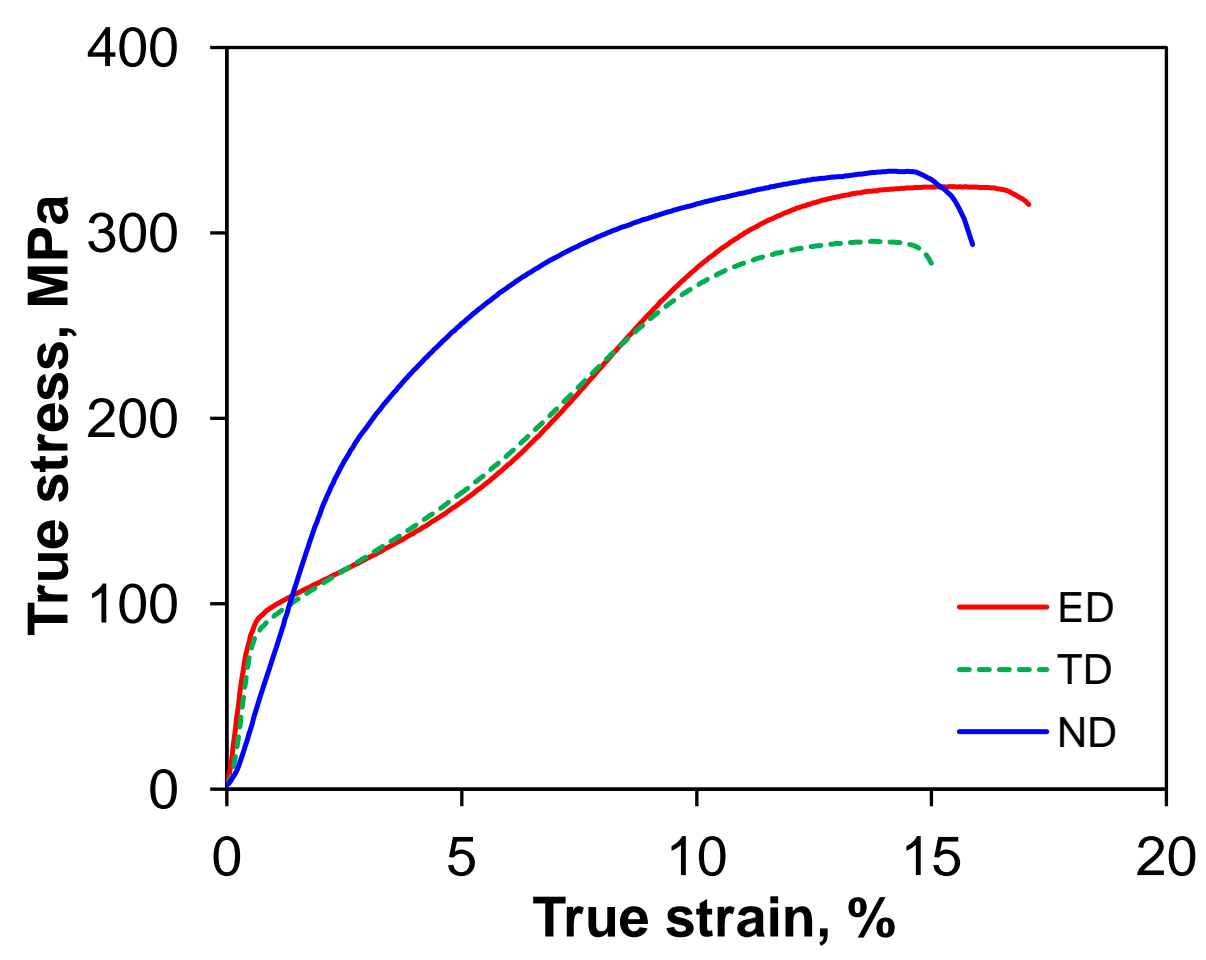

Figure 6.1: True stress-true strain curves of an extruded AM30 along the ED, TD and ND.

\subsection{Effect of Pre-Strain and Loading Direction on Compressive Properties}

The true stress-true strain behavior of the samples that were subjected to different amounts of pre-strains along the ED is shown in Fig. 6.2(a), where the re-compression was also conducted along the ED, as symbolized by $x \% \mathrm{ED}-\mathrm{ED}$. It is of interest to see that a marked change in the sigmoidal shape of the true stress-true strain curves occurred. With increasing pre-strain from $0 \% \mathrm{ED}, 1.5 \% \mathrm{ED}, 2.3 \% \mathrm{ED}, 4.0 \% \mathrm{ED}, 5.8 \% \mathrm{ED}, 7.5 \% \mathrm{ED}$ and $8.2 \% \mathrm{ED}$, the skewed curve shape gradually vanished. When the pre-strain reached about 7.5-8.2\%, the true stress-true strain curve looked like a normal one (e.g., for the fcc and/or bcc metals). The fracture strain of the prestrained (ED-ED) samples also decreased with increasing pre-strain. Fig. 6.2(b) illustrates the 
true stress-true strain curves corresponding to the pre-compression along the ED and recompression along the TD (denoted as $x \%$ ED-TD compressed samples). Obviously, the true stress-true strain curves exhibited different flow characteristics when re-compressed along the TD, compared to the ED-ED compressed sample (Fig. 6.2(a)). Even at a pre-strain of 5.2\% or $7.5 \%$, the skewed/sigmoidal shape of the true stress-true strain curves still remained in the EDTD compressed samples, although the extent of the skewness was reduced. Again, such peculiar shapes were consistent with the twin formation, twin-twin and twin-dislocation interactions, leading to the distinctive strain hardening in compression $[98,129,135]$. A significant change in the shape of the true stress- true strain curve became noticeable when re-compressed along the ND where the sample was pre-compressed along the ED as well, shown in Fig. 6.2(c) (denoted as $x \%$ ED-ND compressed samples). Without pre-straining compression through the ND showed a higher yield stress of $\sim 175 \mathrm{MPa}$ which decreased to $\sim 91 \mathrm{MPa}$ when different amounts of prestrains were applied. With increasing pre-strain from $2.1 \% \mathrm{ED}, 3.7 \% \mathrm{ED}, 5.4 \% \mathrm{ED}$, and 7.9\%ED, the skewed curve shape gradually became more obvious. When the pre-strain reached about 5.4\%-7.9\%, the true stress-true strain curve looked like a twin dominated one. A slight increase in ultimate compressive strength and decrease in fracture strain with increasing pre-strain amount were also observed. In comparison with the ED, TD and ND compression test results of the asextruded samples without pre-deformation (Fig. 6.1), the compressive yield stress clearly increased in the ED-ED and ED-TD compressed samples (Fig. 6.2(a) and (b)), whereas it decreased in the ED-ND compressed sample with increasing pre-strain (Fig. 6.2(c)). The evaluated results were plotted in Fig. 6.3(a). 

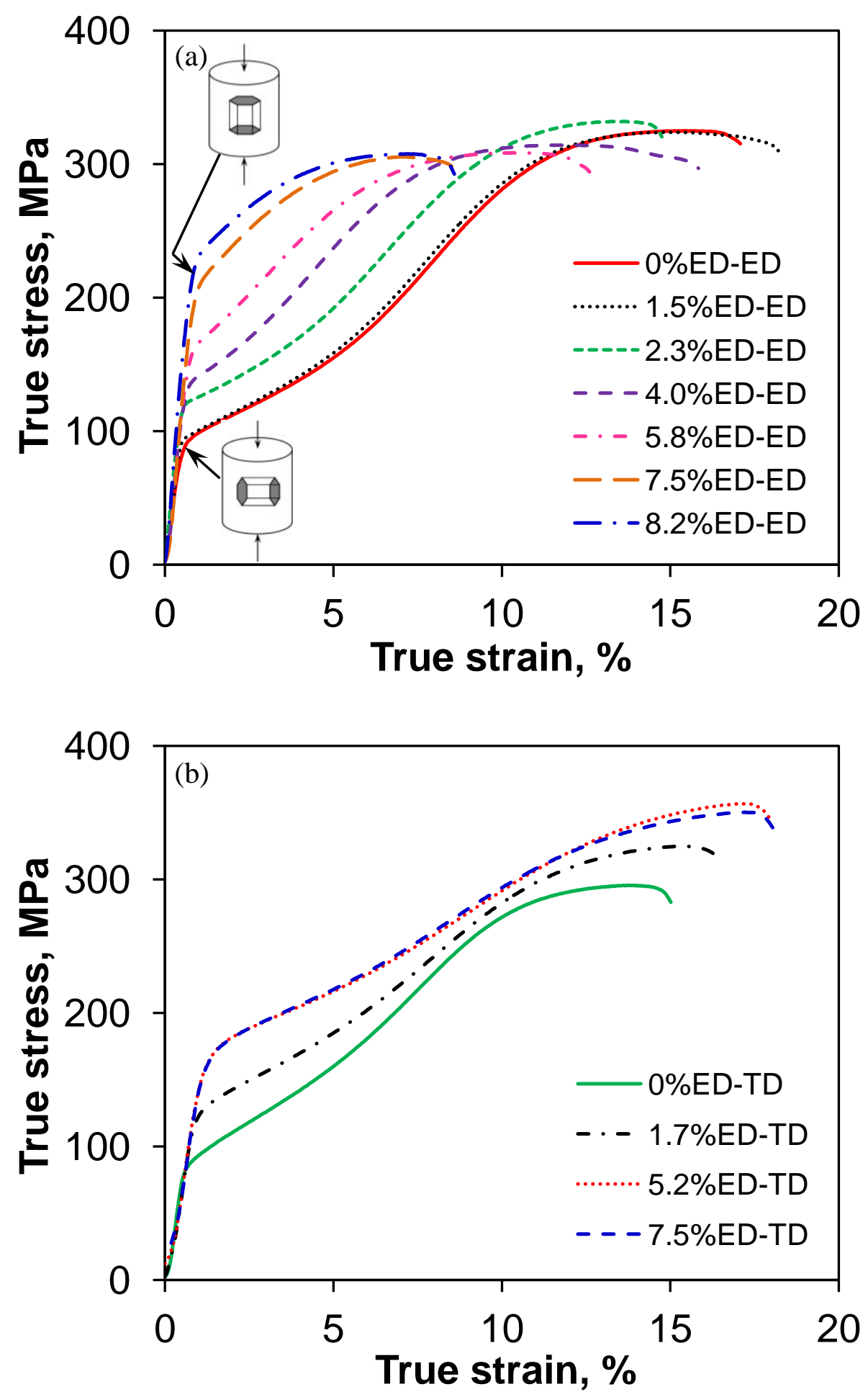


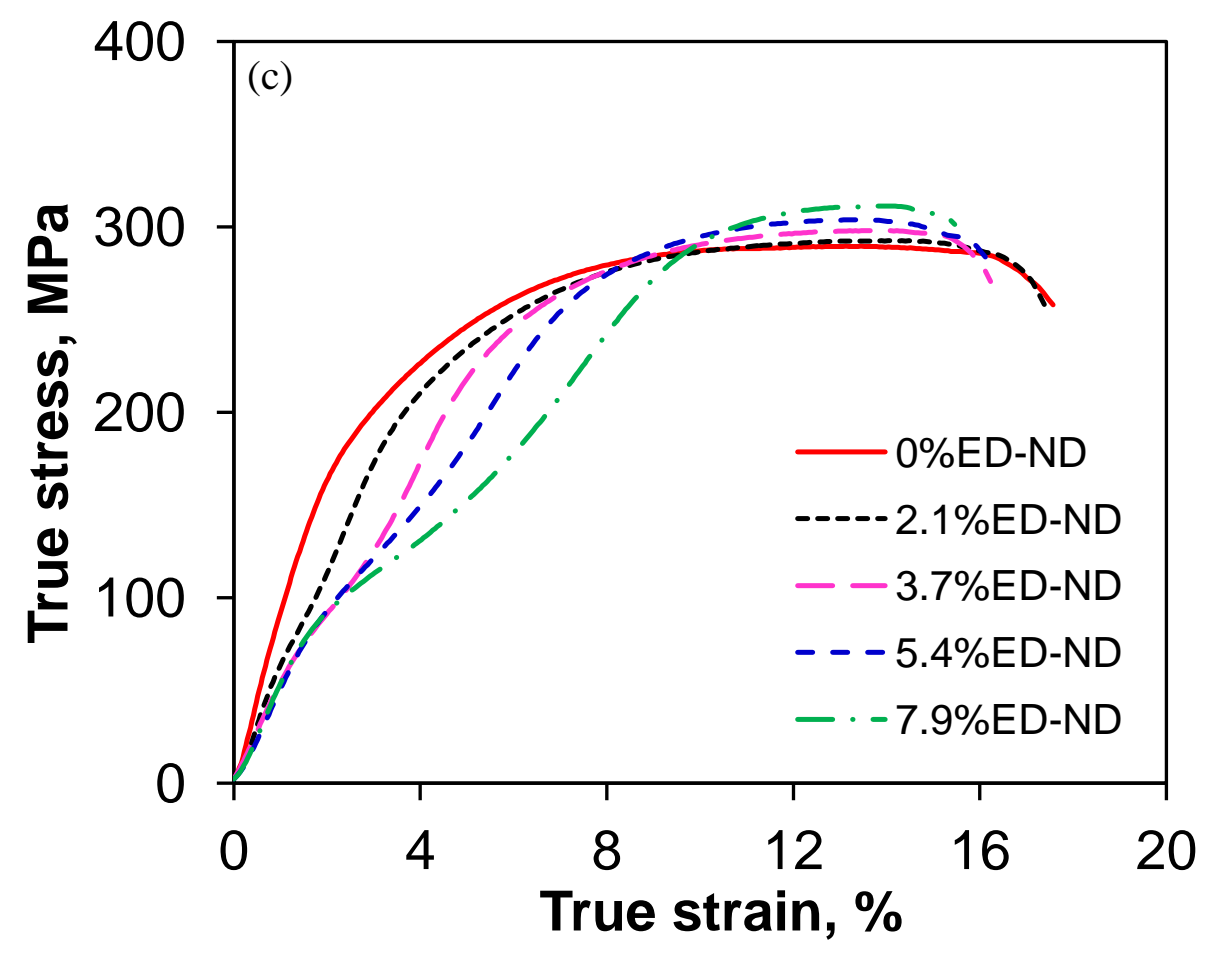

Figure 6.2: $(a, b, c)$ True stress-true strain curves obtained in re-compression along the ED, TD and ND, respectively, after pre-compression at various strain levels along the ED.

In the ED-ED compressed samples, with increasing pre-strain from $1.5 \% \mathrm{ED}, 2.3 \% \mathrm{ED}, 4.0 \% \mathrm{ED}$, 5.8\% ED, 7.5\% ED and 8.2\% ED, the compressive yield stress almost linearly increased to 101 MPa, $119 \mathrm{MPa}, 137 \mathrm{MPa}, 161 \mathrm{MPa}, 202 \mathrm{MPa}$ and $232 \mathrm{MPa}$, respectively, which could thus be expressed by the following linear equation,

$$
\sigma_{y s}=a_{1}+b_{1} \varepsilon_{p r e},
$$



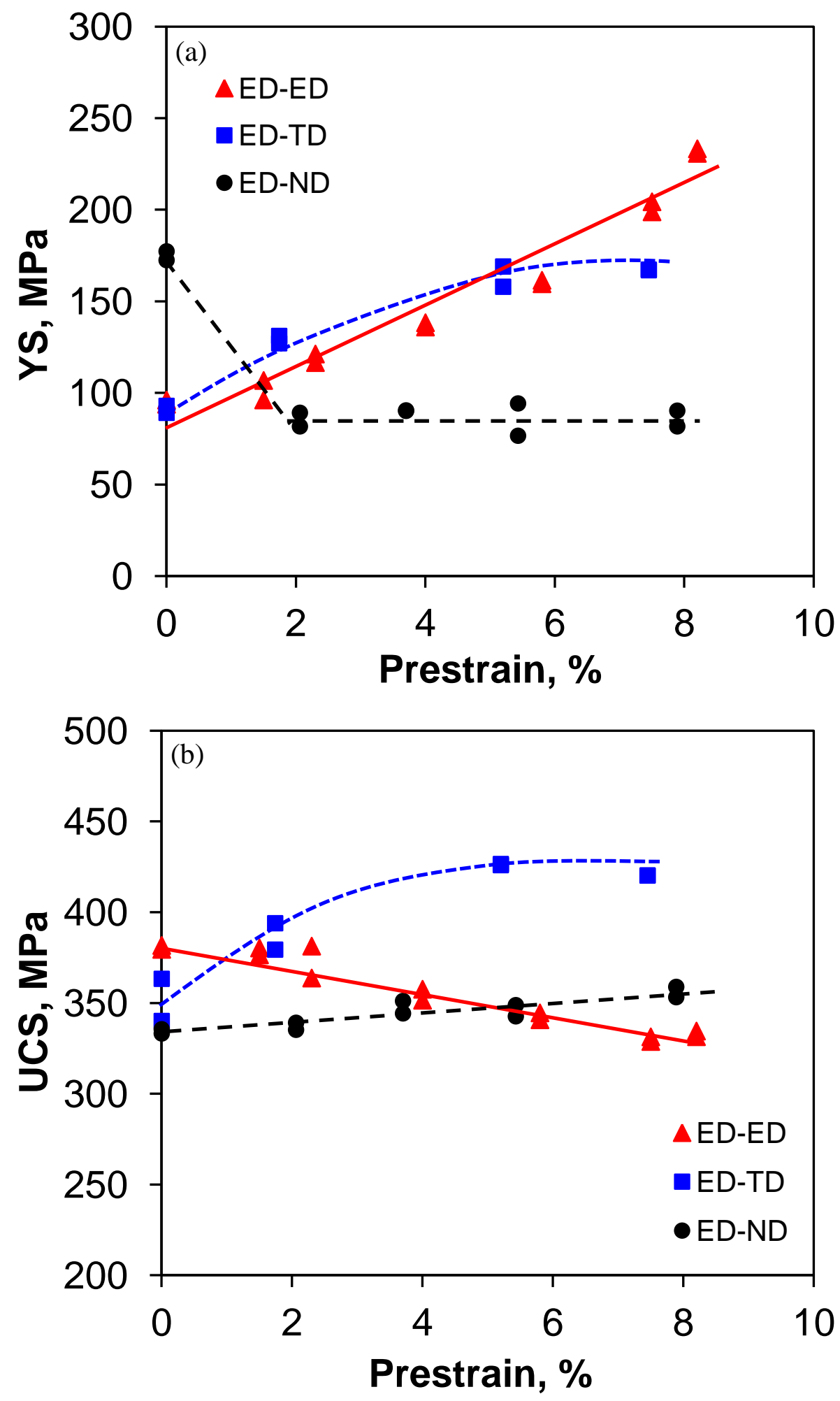


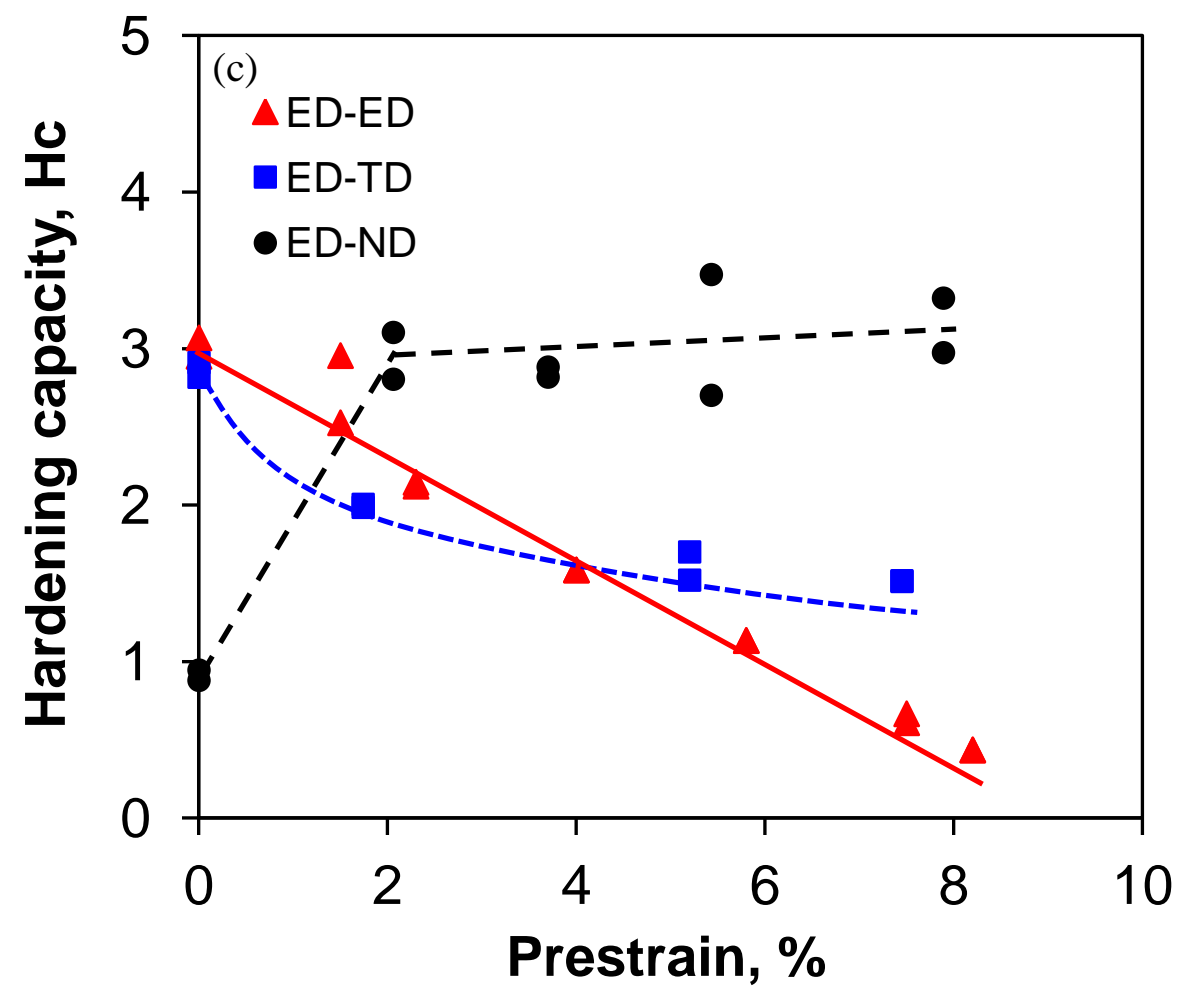

Figure 6.3: Effect of pre-strain along the ED on (a) compressive yield strength (YS), (b) ultimate compressive strength (UCS), and (c) hardening capacity of re-compressed samples along the ED, TD and ND.

where $\sigma_{y s}$ is the compressive yield stress of the pre-strained samples obtained during recompression, $\varepsilon_{p r e}$ is the compressive pre-strain applied, $a_{1}$ and $b_{1}$ are two empirical material constants ( $a_{1}=82 \mathrm{MPa}$ and $b_{1}=1618 \mathrm{MPa}$ obtained for the AM30 alloy in the present study).

In contrast, in the ED-TD compressed samples the compressive yield stress increased nonlinearly with increasing pre-strain. When the applied pre-strain exceeded about 5.2\%, the ED-TD compressed samples showed a plateau or saturation behavior in the compressive yield stress (Fig. 6.3(a)). On the other hand, in the ED-ND compressed samples the yield stress sharply dropped to 
$91 \mathrm{MPa}$ with pre-strain amount of $2.1 \%$ and then showed a constant behavior with further increase of pre-strain (Fig. 6.3(a)). Fig. 6.3(b) shows the ultimate compressive strength (UCS) as a function of pre-strain in the ED-ED, ED-TD and ED-ND compressed samples. It is clear that the effect of pre-strain on the UCS was strongly dependent on the strain path (or loading direction) of re-compression. The UCS of the ED-TD compressed samples initially increased with increasing pre-strain and later reached a plateau as well, which indeed exhibited a similar trend to that of the YS (Fig. 6.3(a)). In ED-ND compressed samples, a slight increase in the UCS with pre-strain is observed. However, the UCS of the ED-ED compressed samples decreased linearly as the pre-strain increased, which could be described by,

$$
\sigma_{u c s}=a_{2}+b_{2} \varepsilon_{\text {pre }}
$$

where $\sigma_{u c s}$ is the ultimate compressive strength of the pre-strained samples obtained during recompression, $a_{2}$ and $b_{2}$ are two empirical constants $\left(a_{2}=384 \mathrm{MPa}\right.$ and $b_{2}=-679 \mathrm{MPa}$ attained for the AM30 alloy in the present study).

Such changes would lead to a significant change in the hardening capacity $\left(H_{\mathrm{c}}\right)$, which could be defined as $[168,176]$,

$$
H_{c}=\frac{\sigma_{u c s}-\sigma_{y s}}{\sigma_{y s}} .
$$

The evaluated hardening capacity is shown in Fig. 6.3(c). The hardening capacity of ED-ED and ED-TD compressed samples decreased, though for ED-ND compressed samples, it increased with increasing pre-strain, but had a different varying trend. For the ED-ND compressed sample, the hardening capacity increased initially with pre-strain and showed constant behavior with 
increased pre-strain because both the compressive yield stress and ultimate compressive stress exhibited a linear trend as a function of pre-strain (Fig. 6.3(a) and (b)). While the hardening capacity decreased faster initially and then became slower with increasing pre-strain in the EDTD compressed samples, a linear decrease was again observed in the ED-ED compressed samples, which could be expressed as,

$$
H_{c}=a_{3}+b_{3} \varepsilon_{\text {pre }},
$$

where $a_{3}$ and $b_{3}$ are two empirical constants $\left(a_{3}=3.0\right.$ and $b_{2}=-32.0$ obtained for the AM30 alloy in the present study). Such a linear change of hardening capacity in the ED-ED compressed samples was expected since both the compressive yield stress and ultimate compressive stress displayed a linear change as a function of pre-strain (Fig. 6.3(a) and (b)). These changes in the mechanical properties were attributed to the change in the microstructure and texture which will be discussed later.

The strain hardening rate $d \sigma / d \varepsilon$ (where $\sigma$ is true stress) of the ED-ED, ED-TD and ED-ND compressed samples as a function of true strain $\varepsilon$, is plotted in Fig. 6.4. In the case of ED-ED compressed samples (Fig. 6.4(a)), at a pre-strain along the ED from 0 to 5.8\% three distinct stages of strain hardening can be distinguished, which are also illustrated schematically in the inset of Fig. 6.4(a), where stage A was characterized by a rapidly falling strain hardening rate, followed by stage $\mathrm{B}$ with an increasing strain hardening rate, and then stage $\mathrm{C}$ with decreasing strain hardening rate again until failure. Similar trends in the strain hardening rates have also been observed in $\alpha$-titanium [127] and AZ31 magnesium alloy [128], and AM30 magnesium alloy compressed without pre-straining [135]. It is of special interest to note that the extent of 
stage B decreased with increasing pre-strain, and stage B disappeared completely when the prestrain surpassed a pre-strain of 7.5\%, as indicated by an upward dashed-line arrow in Fig. 6.4(a). That is, at a pre-strain of $7.5 \%$ and $8.2 \%$, stage $\mathrm{C}$ emerged immediately after the rapidly falling stage A in the absence of stage B. From Fig. 6.4(a) it is seen that stage C in this case could be approximated by a linear decrease with increasing true strain, which indeed reflected stage III hardening in the tensile deformation $[12,176]$. Also, stage $\mathrm{C}$ was observed to move downward with increasing pre-strain, as indicated by a downward solid-line arrow in Fig. 6.4(a). Similarly, three characteristic stages of strain hardening appeared after re-compression along the TD, where the pre-strain was applied along the ED as well, as seen from Fig. 6.4(b). While the degree of stage B in this case decreased with increasing pre-strain, stage B did not fade away completely even at a pre-strain of $7.5 \%$. In this case, the value of peak strain hardening rate decreased, with a decreasing slope of both stage B and stage $\mathrm{C}$, which made both stages flatten, as indicated by the downward dashed-line arrow and upward solid-line arrow in Fig. 6.4(b), respectively. Similar hardening behavior was observed in a hot-rolled AZ31 magnesium alloy where samples were pre-compressed along the rolling direction to a plastic strain of $1.8 \%, 3.4 \%$ and $6 \%$ and recompressed along the TD [20]. To characterize the varying degrees of stage $\mathrm{B}$, the change range of strain hardening rate in stage $\mathrm{B}\left(\Delta \theta_{\mathrm{B}}\right.$, defined in the inset of Fig. 6.4(a)) was evaluated and plotted in Fig. 6.4(c) for both ED-ED and ED-TD compressed samples, where $\Delta \theta_{\mathrm{B}}$ is defined as the peak/maximum strain hardening rate between stages $\mathrm{B}$ and $\mathrm{C}$ minus the valley/minimum strain hardening rate between stages A and B shown in Fig. 6.4(a) and (b). 

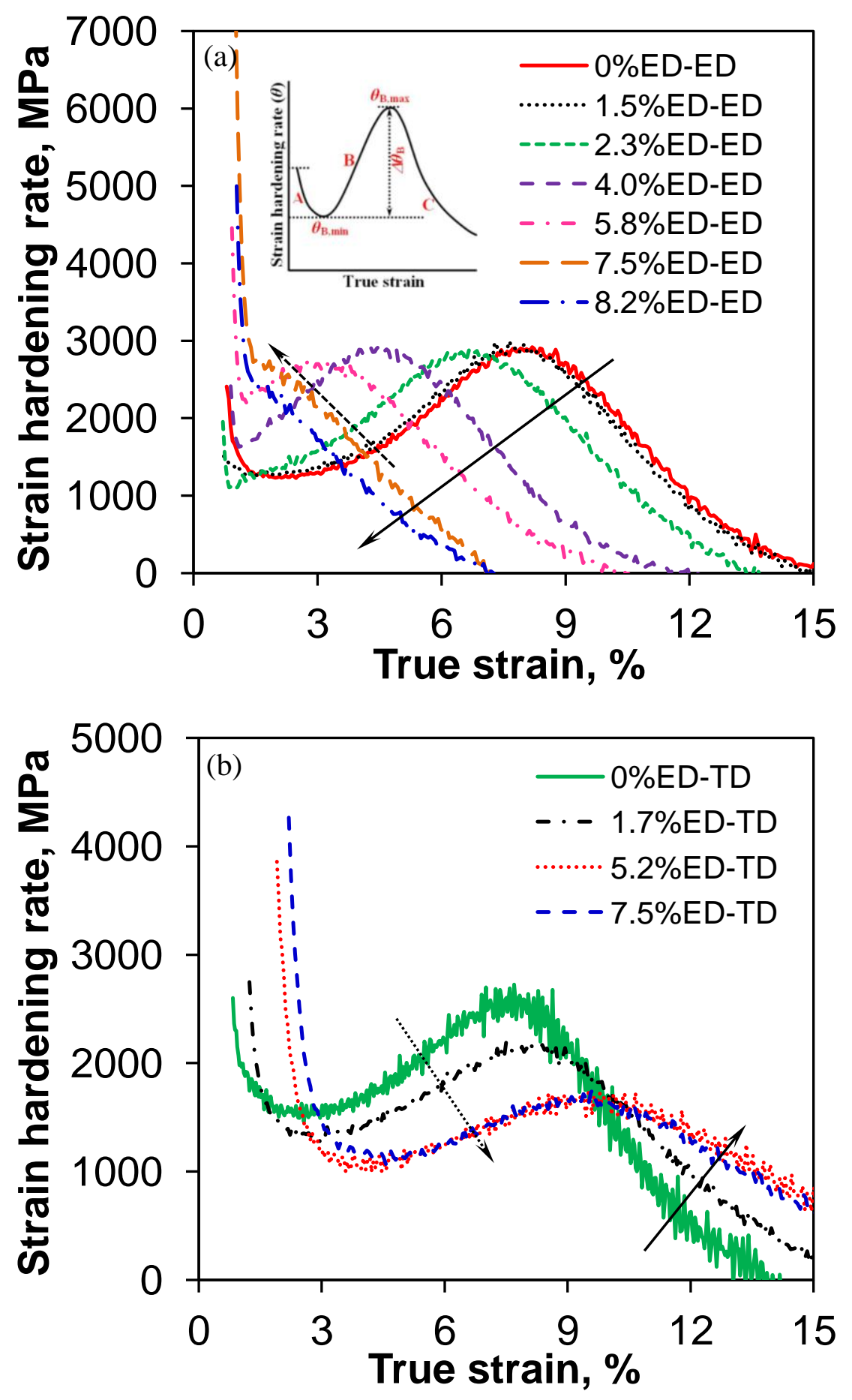

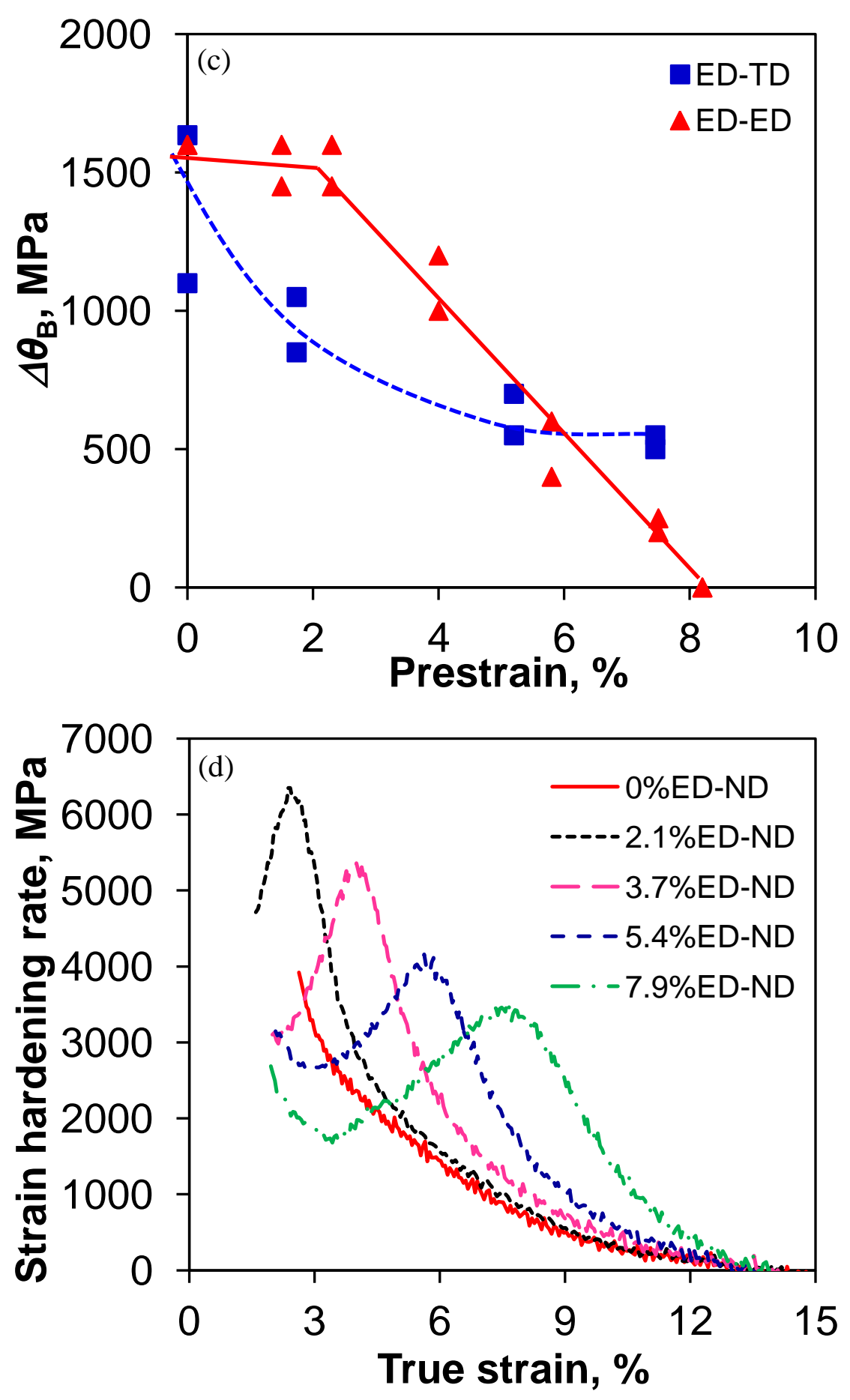

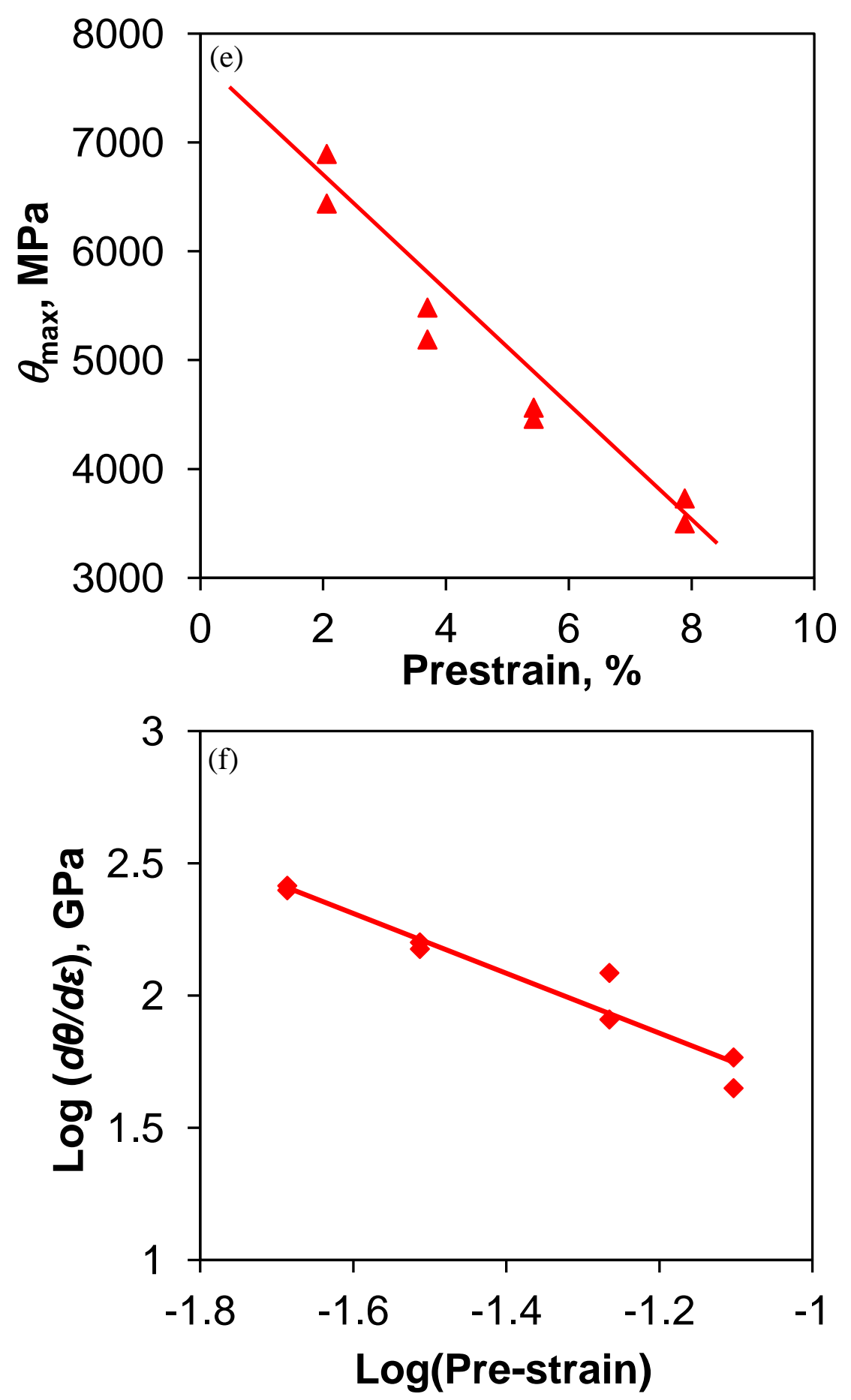

Figure 6.4: For the ED-ED and ED-TD compressed samples, $(a, b)$ strain hardening rate vs. true strain respectively, (c) change range of strain hardening rate in stage $\mathrm{B}\left(\Delta \theta_{\mathrm{B}}\right)$ as a function of pre-strain. For the ED-ND compressed samples, (d) strain hardening rate vs. true strain, (e) peak value of strain hardening rate, $\theta_{\max }$ vs. pre-strain, (f) $\log (d \theta / d \varepsilon)$ vs. Log (pre-strain). 
It is seen from Fig. 6.4(c) that when the pre-strain was below 2.3\%, the ED-ED compressed samples showed a nearly constant $\Delta \theta_{\mathrm{B}}$ value of $\sim 1600 \mathrm{MPa}$, followed by a linear drop with increasing pre-strain until zero at a pre-strain of $8.2 \%$. In the case of the ED-TD compressed samples, a decreasing trend of $\Delta \theta_{\mathrm{B}}$ was also obvious with increasing pre-strain. However, the value of $\Delta \theta_{\mathrm{B}}$ tended to level off at a higher level of pre-strain, corresponding well to the decreasing hardening capacity (Fig. 6.3(c)). The diagram shown in Fig. 6.4(c) clearly indicated a significant difference of stage $B$ hardening behavior between the ED-ED and ED-TD compressed samples which were in essence due to the orientation effect that governed the formation and growth of extension twins, which will be presented in the next section.

On the other hand, different stages of strain hardening can be distinguished in the ED-ND compressed samples, as shown in Fig. 6.4(d). In the absence of pre-straining, compression along the ND resulted in a linear decrease in the hardening curve, which reflected stage III hardening followed by stage IV hardening in the tensile deformation [177-179]. In the case of the lower pre-strain level of $2.1 \%$ and $3.7 \%$, two distinct stages (i.e., stages B and C) were obvious in the hardening curve which changed to three distinct stages (i.e., stages A, B and C) with increasing pre-strain to $5.4 \%$ and $7.9 \%$. A similar trend in the strain hardening rate has also been observed in hep materials, such as $\alpha$-titanium [127], Zr-1Nb zirconium alloy [180], AZ31 magnesium alloy [128], and AM30 magnesium alloy [135,169]. It is also seen from Fig. 6.4(d) that the range of stage B became wider with increasing pre-strain amount. While stage A was associated with the formation of $\{10 \overline{1} 2\}$ extension twins during compression, the ascending stage B was mainly attributed to the twin-twin and twin-dislocation interactions and the resulting twin growth which will be discussed later. To characterize the strain hardening rate, the value of peak strain 
hardening rate $\left(\theta_{\max }\right)$ at different pre-strain levels was evaluated and plotted in Fig. 6.4(e), where $\theta_{\max }$ is defined as the peak/maximum strain hardening rate between stages B and C. With increasing pre-strain from $2.1 \% \mathrm{ED}, 3.7 \% \mathrm{ED}, 5.4 \% \mathrm{ED}$, and $7.9 \% \mathrm{ED}$, the average $\theta_{\max }$ value decreased almost linearly from $6667 \mathrm{MPa}$ to $5336 \mathrm{MPa}, 4512 \mathrm{MPa}$, and $3614 \mathrm{MPa}$, respectively, which could be expressed by the following equation,

$$
\theta_{\max }=a_{4}+b_{4} \varepsilon_{p r e}
$$

where $\theta_{\max }$ is the value of peak strain hardening rate obtained during re-compression after predeformation, $\varepsilon_{\text {pre }}$ is the compressive pre-strain, $a_{4}$ and $b_{4}$ are two empirical material constants $\left(a_{4}\right.$ $=7239 \mathrm{MPa}$ and $b_{4}=-47838 \mathrm{MPa}$ obtained for the extruded AM30 alloy in the present study). The slope of the strain hardening rate in stage B $(d \theta / d \varepsilon)$ was also evaluated from the strain hardening curves (Fig. 6.4(d)), and the obtained $\log (d \theta / d \varepsilon)$ vs. Log (pre-strain) is plotted in Fig. 6.4(f). With increasing pre-strain amount from $2.1 \% \mathrm{ED}$ to $7.9 \% \mathrm{ED}$, a linearly decreasing trend in the slope is observed in the double-log scales, which could be expressed by the following equation,

$$
\frac{d \theta}{d \varepsilon}=K \varepsilon_{p r e}^{b},
$$

where $d \theta / d \varepsilon$ is the slope of the strain hardening rate in stage $\mathrm{B}$, obtained after the second step compression in the ND of the ED pre-strained sample, $\varepsilon_{\text {pre }}$ is the compressive pre-strain in the $\mathrm{ED}$, and $K$ and $b$ are two empirical material constants $(K=3.2 \mathrm{GPa}$ and $b=-1.1 \mathrm{GPa}$ obtained for the extruded AM30 alloy in the present study). Such a linear change in the slope of strain hardening rate clearly indicated a significant difference of stage B hardening behavior, which was expected since the compressive yield stress displayed a large decrease (from 175 $\mathrm{MPa}$ to 
291 MPa in Fig. 6.3(a)) and a slight increase in the ultimate compressive strength (Fig. 6.3(b)) as

a function of pre-strain. These distinctive macroscopic deformation characteristics were attributed to the change in the microstructure and texture which will be discussed later.

\subsection{Effect of Pre-strain and Loading Direction on Microstructure}

Microstructure evolution was examined by optical microscopy for the ED-ED, ED-TD and EDND compressed samples at different pre-strain levels. The microstructure shown in Fig. 6.5(a), corresponding to a compressive strain of $4.0 \%$ along the ED was very different from the initial microstructure shown in Fig. 4.1(a). At this amount of strain, almost all grains were subjected to extension twinning which traversed the parent grains and interacted with each other. With a continuous deformation to a compressive strain of $8.2 \%$ along the ED, as shown in Fig. 6.5(b), most twin boundaries apparently disappeared or only some traces of twin boundaries in the microstructure were visible, indicative of twin growth. As described earlier, during compression the nucleation and growth of $\{10 \overline{1} 2\}$ extension twinning were very fast and also influenced by the interaction of dislocations with twin boundaries, depending on the characteristics of dislocations and the driving stress [32,118,119,135].

To observe the effect of pre-strain on the twin formation, a $4.0 \%$ re-compression along the ED and TD was carried out, respectively, after a $4.0 \%$ pre-strain along the ED, and the obtained microstructure is shown in Fig. 6.6(a) and (b). 

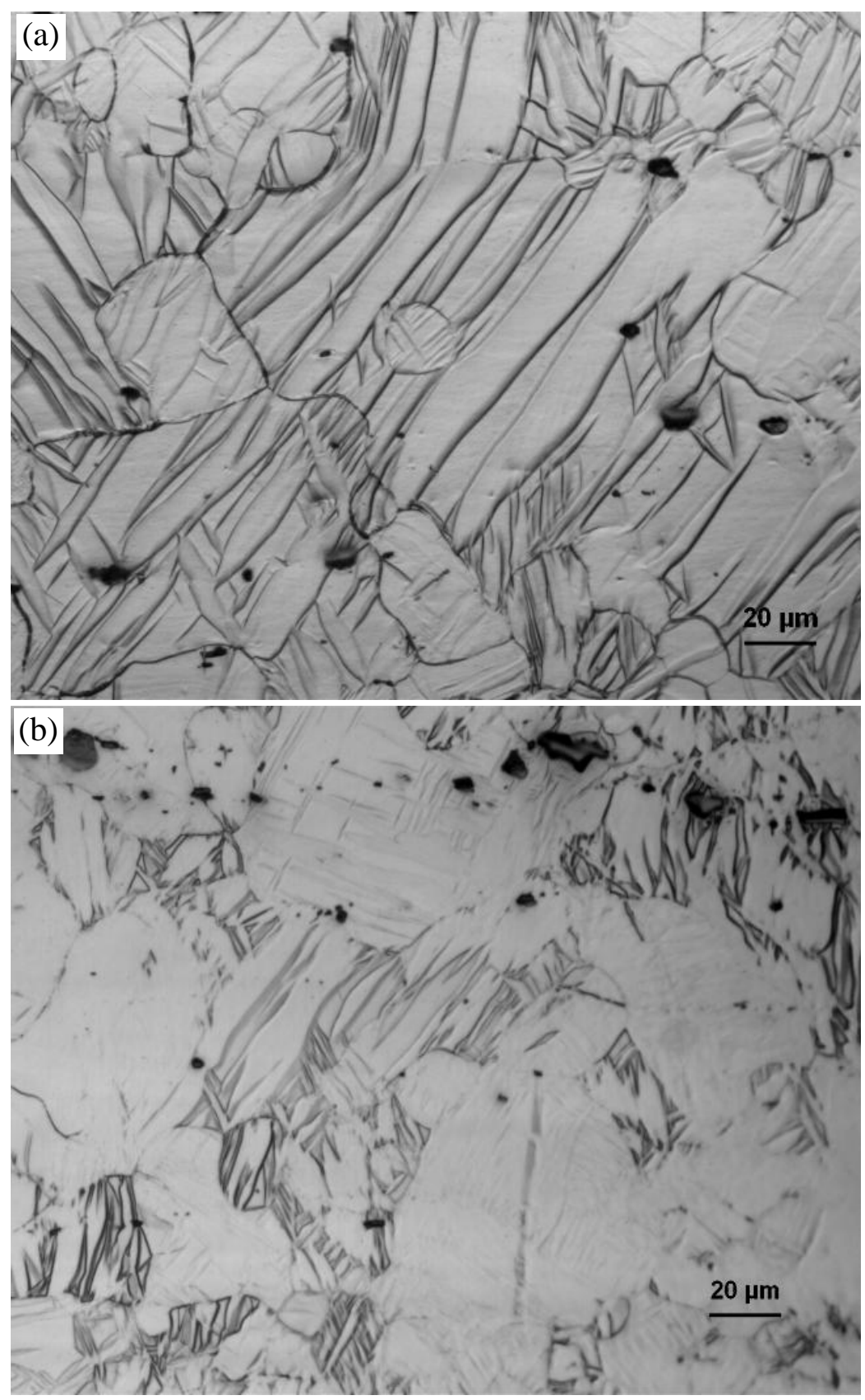

Figure 6.5: Interior microstructures of the AM30 samples subjected to a continuous compressive strain of (a) $4.0 \%$ and (b) $8.2 \%$ along the ED at a strain rate of $1.25 \times 10^{-4} \mathrm{~s}^{-1}$ and room temperature. 
For the ED-ED compressed samples (Fig. 6.6(a)), the vanishing of some twin boundaries or the coalescence of twins by twin growth was observed, in comparison with the microstructure in Fig. 6.5(a). This situation was somewhat similar to Fig. 6.5(b).

However, the twin boundaries in Fig. 6.6(a) were more visible within the pre-existing twins due to the effect of pre-strain, suggesting that the extent of twin growth was lower in comparison with a continuous compression to $8.2 \%$ (Fig. 6.5(b)). On the other hand, a $4.0 \%$ re-compression along the TD after a $4.0 \%$ pre-strain along the ED caused more secondary twin formation and eventually refined grain size as shown in Fig. 6.6(b). It should be noted that the above observations involved the interior microstructures of the compressed samples. To better identify the effect of re-compression direction on the twinning, a sample was first pre-polished and then a two-step compressive deformation test was done as well. Fig. 6.7(a) and (b) show the twin appearance in the same area on the pre-polished sample surface after $4.0 \%$ pre-strain along the ED and after $4.0 \%$ pre-strain along the ED plus $4.0 \%$ re-compression along the TD. It is of particular interest to observe that the width of some twins formed in the first step became narrower or their length became shortened in the same grains after $4.0 \%$ re-compression along the TD, as indicated by the red dashed ovals in Fig. 6.7. This indicates that a change in the recompression direction led to the occurrence of de-twinning in some grains oriented favorably. On the other hand, new twins were also observed to form in some grains after $4.0 \%$ recompression along the TD, as indicated by the blue arrows in Fig. 6.7(b), corresponding well to the situation shown in Fig. 6.6(b). Therefore, in the present study two phenomena were observed to be co-existent in the case of the re-compression along the TD after pre-strain along the ED: (1) the formation of new twins within the twinned and un-twinned regions (Fig. 6.6(b) and Fig. 
6.7(b)), (2) and the occurrence of de-twinning (Fig. 6.7). This will be further verified by the texture measurements.
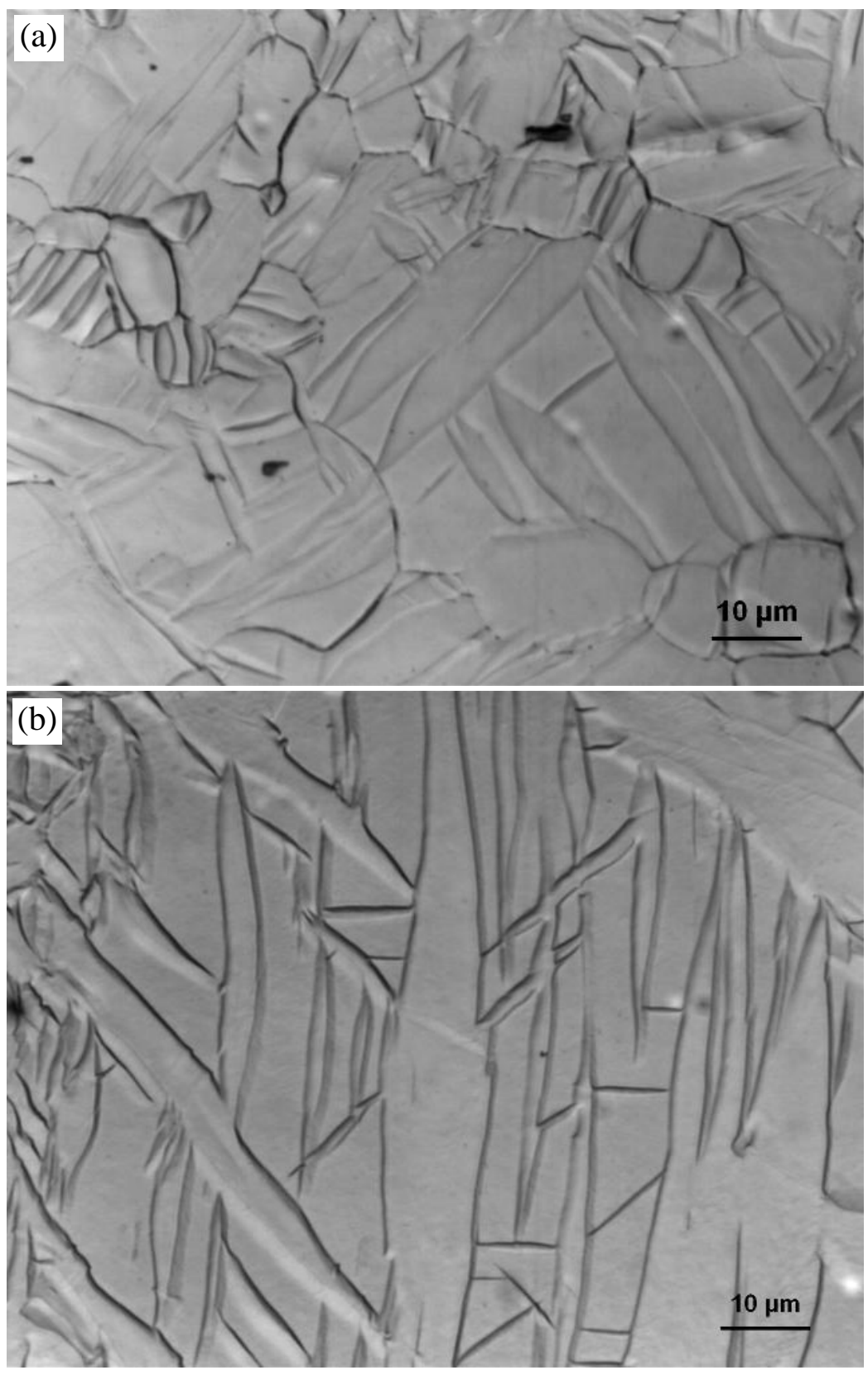

Figure 6.6: Interior microstructures of the AM30 samples subjected to a two-step compressive deformation, (a) 4.0\% re-compression along the ED and (b) $4.0 \%$ re-compression along the TD, after a $4.0 \%$ pre-strain along the ED. 


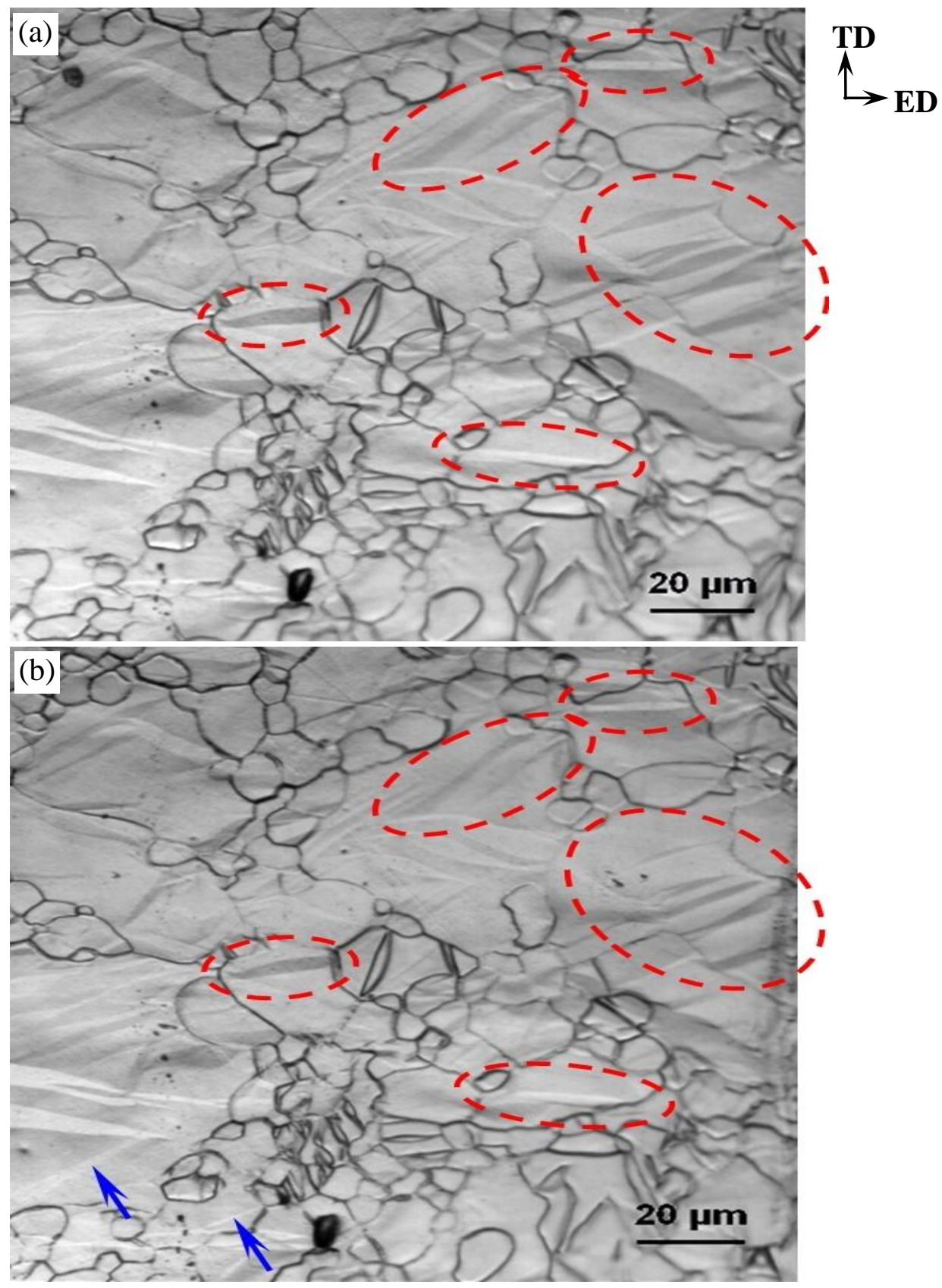

Figure 6.7: Change of microstructures on the pre-polished surface of an AM30 sample subjected to (a) $4.0 \%$ pre-strain along the ED only, and (b) $4.0 \%$ pre-strain along the ED plus $4.0 \%$ recompression along the TD, where the red dashed ovals denote de-twinning or twin shrinkage (narrowing, shortening), and the blue arrows indicate the formation of new twins. 
Fig. 6.8 shows the microstructure evolution examined by optical microscopy for samples that were first compressed along the ED at different pre-strain levels, which were then re-compressed along the ND at a fixed strain level of 3.7\%. It is seen from Fig. 6.8(a), corresponding to a compressive strain of $1.7 \%$ along the $\mathrm{ED}$, that almost all grains were subjected to extension twinning, where the twins traversed the parent grains and interacted with each other. To observe the effect of pre-strain on the microstructure, the $2.1 \%$ compressed sample along the ED was recompressed at a strain of 3.7\% along the ND and is shown in Fig. 6.8(b), where the twins disappeared from almost all grains and only very few traces of twins in the microstructure were visible, indicative of the occurrence of nearly complete de-twinning. With deformation to a compressive strain of $4.0 \%$ along the ED, as shown in Fig. 6.8(c), twins started to become wider as compared to Fig. 6.8(a) and their boundaries merged with each other, due to the twin growth [169]. In this case a further $3.7 \%$ recompression along the ND also caused de-twinning, which was obvious from some twin free grains, whereas other grains showed narrower/thinner twins as seen in Fig. 6.8(d). At a higher strain to 8.2\% along the ED, as shown in Fig. 6.8(e), most twin boundaries apparently disappeared and only some traces of twins were visible as they encompassed the whole grain. After the second-step compression along the ND of the previously compressed sample at a pre-strain amount of $7.9 \%$ twin shrinkage was observed by the narrowing of the existing twins as seen in Fig. 6.8(f) vs. Fig. 6.8(e). The observation clearly indicated that with increasing pre-strain amount while keeping the re-compression strain amount constant, the de-twinning tendency decreased or became more difficult as was evident from the thin narrow twins observed in most grains. This will be further verified through the texture measurements. 

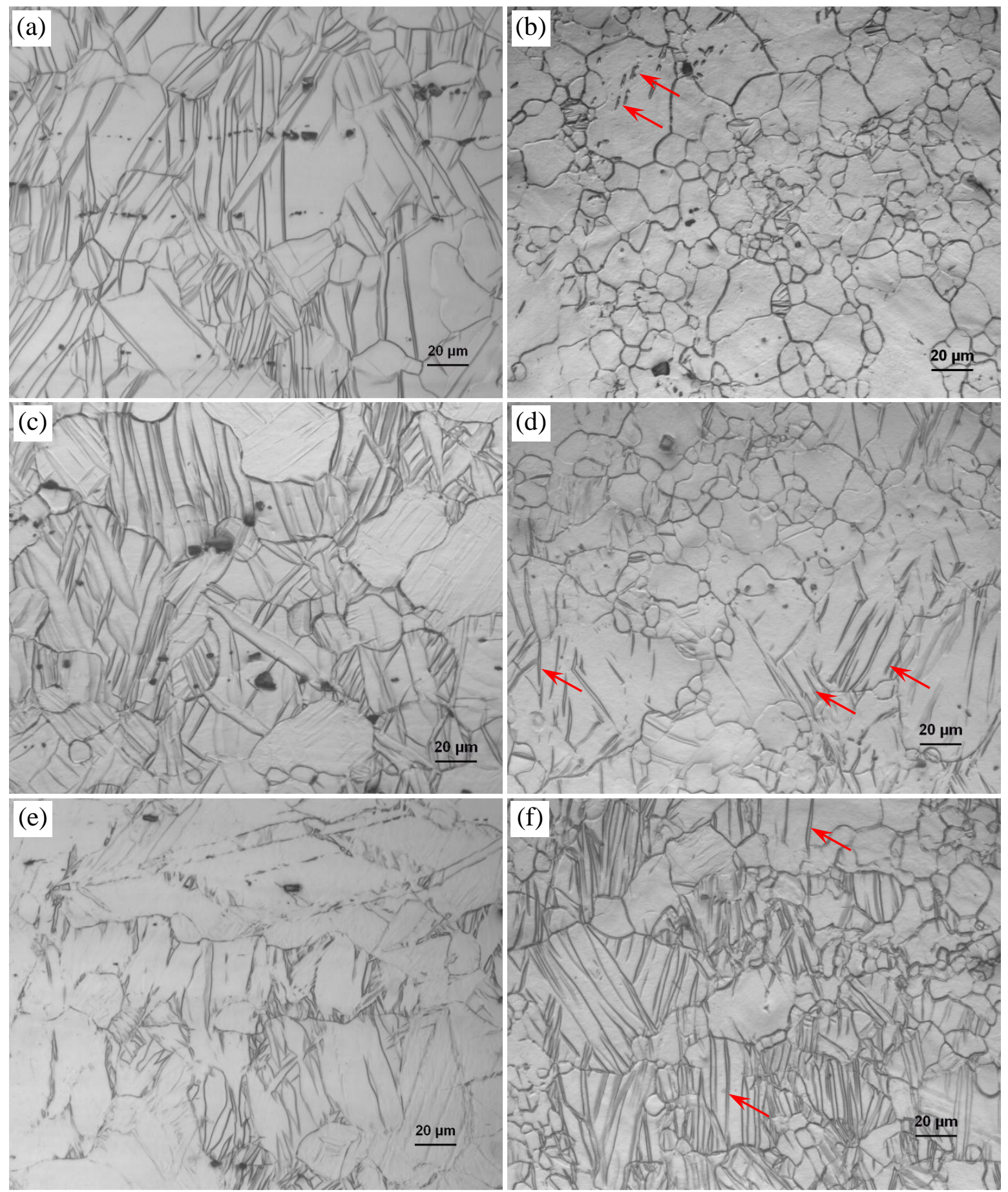

Figure 6.8: Microstructures of the compressed AM30 samples at a strain of (a) $1.7 \%$ along ED,

(b) $3.7 \%$ re-compression along ND after $2.1 \%$ pre-strain along ED, (c) $4.0 \%$ along ED, (d) $3.7 \%$ re-compression along ND after 3.7\% pre-strain along ED, (e) $8.2 \%$ along ED, (f) $3.7 \%$ recompression along ND after $7.9 \%$ pre-strain along ED. 

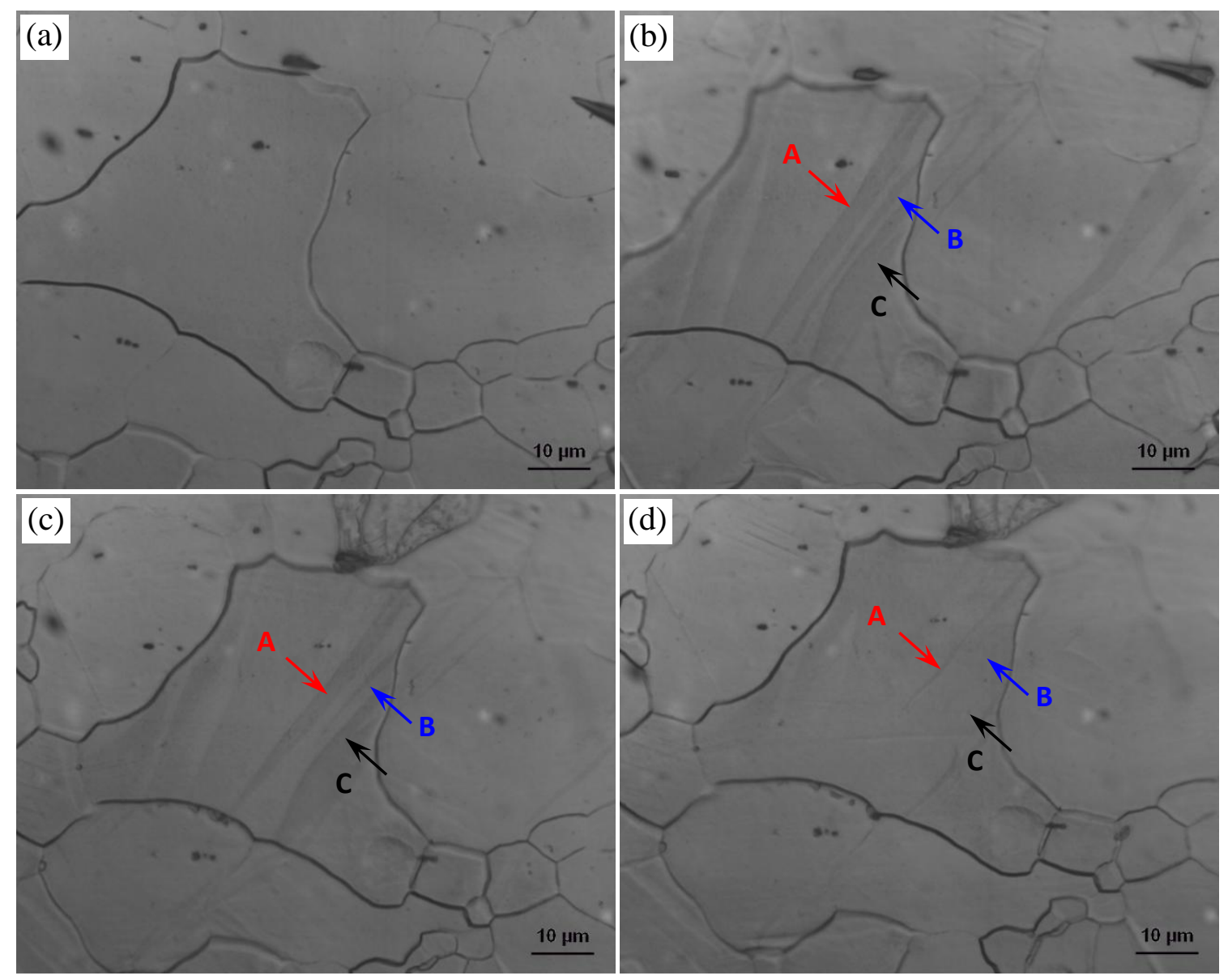

Figure 6.9: (a) Initial microstructure and twin width during stepwise compression after a cumulative strain amount of (b) $3.7 \% \mathrm{ED}$, (c) $3.7 \% \mathrm{ED}-3.5 \% \mathrm{ND}$, and (d) $3.7 \% \mathrm{ED}-7.7 \% \mathrm{ND}$.

To better identify the effect of changing the loading direction during re-compression on twin shrinkage, a sample was first pre-polished and then a two-step compressive deformation test was done. Twin free grains and the appearance of twins in the same area on the pre-polished sample surface after 3.7\% pre-strain along the ED is shown in Fig. 6.9(a) and (b) respectively. When the stepwise compression along the ND reached a cumulative true strain of $3.5 \%$, narrowing/thinning of the previous twins occurred (Fig. 6.9(c)). A further increase of strain to 7.7\% led to the disappearance of twin boundaries or de-twinning, as shown in Fig. 6.9(d). 


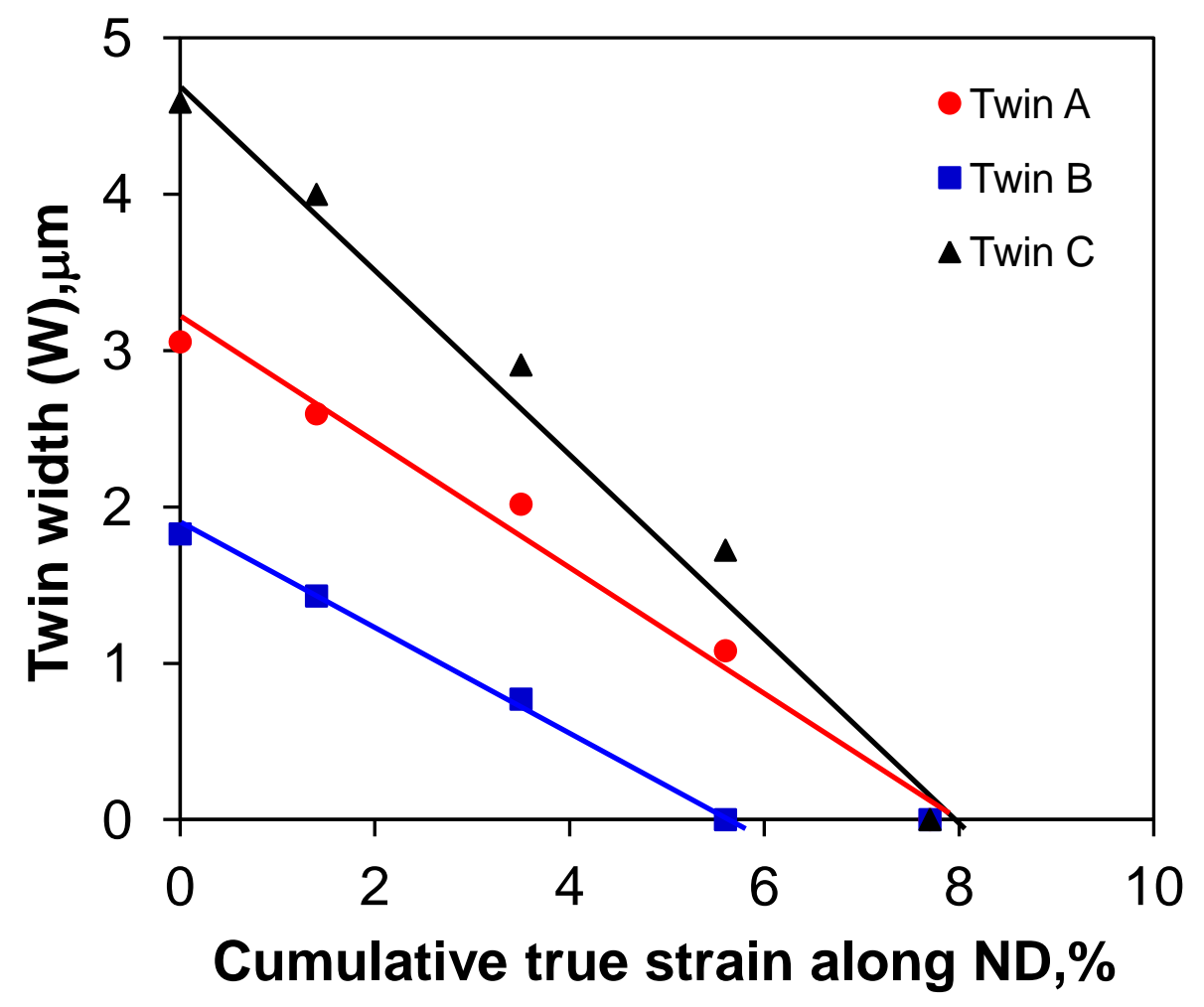

Figure 6.10: Twin width vs. cumulative true strain along the ND of pre-strained sample.

The successive twin shrinkage resulted in complete de-twinning and returned the grain to the initial twin free condition. As the compression causes rotation of the $c$-axis towards the compression direction, after pre-compression along the ED, the $c$-axis would be parallel to the ED which would result in a favorable condition for twinning during reloading along the ND. The interaction of dislocations with twin boundaries that dissociated into twinning dislocations (TDs) can glide through the twin boundary while the opposite motion causes twin shrinkage.

To quantify the twin shrinkage behavior, the change of the width of a few representative twins indicated as "A", "B" and "C" in Fig. 6.9 as a function of cumulative true strain along the ND was measured after each step of re-compression and plotted in Fig. 6.10. After the initial compression along the ED, at a strain of $3.7 \%$, the width of twins $\mathrm{A}, \mathrm{B}$ and $\mathrm{C}$ was $3.06 \mu \mathrm{m}, 1.83$ 
$\mu \mathrm{m}$ and $4.59 \mu \mathrm{m}$ respectively. With increasing re-compressive true strain along the $\mathrm{ND}$, from $1.4 \%$ up to $7.7 \%$, the width of all three twins clearly decreased and eventually vanished completely. It is of interest to note that for all the twins, the width vs. strain exhibited a linearly decreasing trend. The initial width of "C" was larger compared to twins "A" and "B" and showed a faster width reduction with increasing cumulative true strain. The relevant mechanism will be discussed later.

\subsection{Effect of Pre-strain and Loading Direction on Texture Evolution}

Fig. 6.11 shows the (0001) and (1010) pole figures representing the texture of the extruded AM30 in cases of two-step ((a) 4.0\%ED-4.0\%ED, and (b) 4.0\%ED-4.0\%TD) compressive deformation. The one step compression shown in Fig. 4.7(b) and (c) suggests that the $c$-axes of grains were gradually rotated towards the compression direction during the continuous one-step compression. This was expected since the $\{10 \overline{1} 2\}$ extension twinning has been observed to dominate the deformation. The $\{10 \overline{1} 2\}$ extension twinning led to a $86.3^{\circ}$ rotation of basal planes in the twin with respect to the basal planes in the parent grain [19]. This means that the $c$-axes of hcp unit cells in twinned regions were oriented always in the compression direction or aligned along the loading axis (i.e., ED in this case) $[129,135]$. While the same rotation of $c$-axes of grains towards the compression direction in the 4.0\%ED-4.0\%ED two-step compressed sample shown in Fig. 6.11(a)), had a less intense rotation of both basal (0001) poles with an intensity of 9.6 MRD and prismatic (1010) poles with an intensity of 2.7 MRD, which lay just in-between those shown in Figs 4.7(b) and (c) with a continuous/non-interrupted compression. 


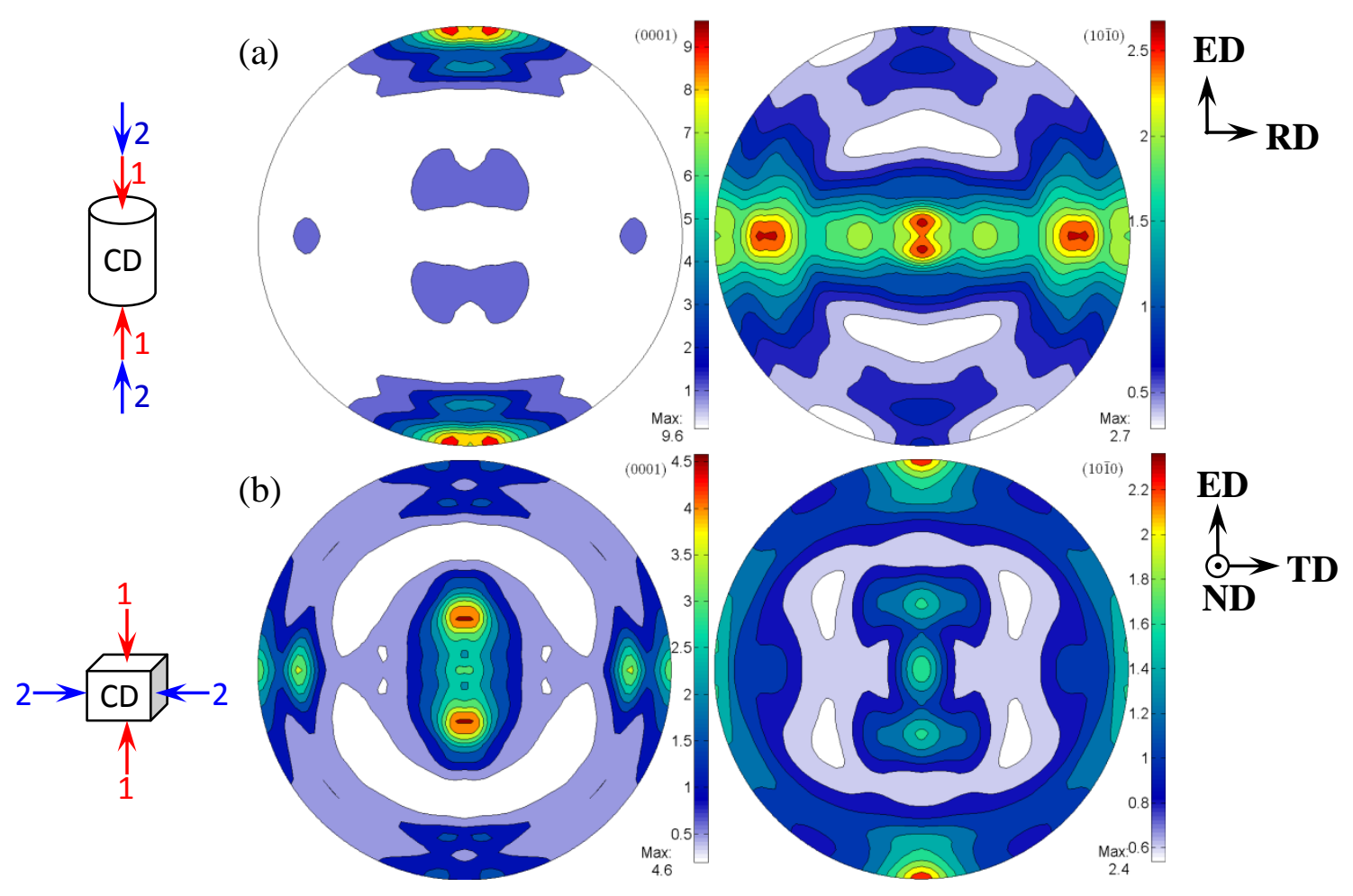

Figure 6.11: (0001) and (10ī0) pole figures of (a) 4.0\%ED-4.0\%ED, and (b) 4.0\%ED-4.0\%TD compressed samples, where "1" stands for the pre-compression direction along the ED, " 2 " denotes the re-compression direction, RD signifies the radial direction of the round samples, and $\mathrm{ED}, \mathrm{TD}, \mathrm{ND}$ indicate the extrusion direction, transverse direction and normal direction of extruded plate, respectively.

This corresponded well to the twinning situation shown in Fig. 6.6(a) which lay in-between those shown in Fig. 6.5(a) and Fig. 6.5(b), as described earlier. The texture shown in Fig. 6.11(a) could be considered to represent a cumulative texture developed via a two-step straining. In comparison with the case of Fig. 4.7(b), the re-compression in the same loading path (ED-ED) led to further twin growth which was evident from the microstructure change shown in Fig. 6.6(a), where the twin boundaries became blurred or disappeared. Interestingly, as seen in Fig. 6.11(b) the same amount (4.0\%) of re-compression but along the TD after $4.0 \%$ pre-strain in the 
ED led to a very different texture in comparison with Fig. 6.11(a), or a significant change in both basal (0001) and prismatic (1010) poles in comparison with the case of $4.3 \%$ pre-compression along the ED (Fig. 4.7(b)). Indeed, such a two-step compression of $4.0 \%$ pre-strain along the ED plus $4.0 \%$ re-compression along the TD resulted in the basal (0001) poles to be reversed back to the center with about $20^{\circ}$ tilting towards the ED with some of them towards the TD as well, which was fairly similar to the texture shown Fig. 4.1(b) in spite of a lower basal pole intensity of 4.6 MRD. This confirms that the second step of compressive deformation along the TD had largely reverted the texture to that in the undeformed as-extruded state, and the $c$-axes of hcp unit cells in most grains once again became nearly parallel to the ND (Fig. 6.11(b) and Fig. 4.1(b)). Therefore, from these observations it is evident that de-twinning occurred in the second step of compressive deformation along the TD, as visibly recognizable in Fig. 6.7(b) by the narrowing or shortening of the pre-existing $\{10 \overline{1} 2\}$ twins in the same grains circled in Fig. 6.7(a).

Fig. 6.12 shows the (0001) and (1010) pole figures representing the texture of the ED-ND compressed samples in different cases of (a), one continuous step ((a) 4.0\%ND), and two step ((b) $2.1 \% \mathrm{ED}-3.7 \% \mathrm{ND}$, (c) $3.7 \% \mathrm{ED}-3.7 \% \mathrm{ND}$, and (d) $7.9 \% \mathrm{ED}-3.7 \% \mathrm{ND}$ ) compressive deformation. The compression along the ND in Fig. 6.12(a) showed a texture position similar to the initial texture but with a stronger intensity of 9.7 MRD for the basal (0001) poles and 2.8 MRD for the (1010) poles, in comparison with the un-deformed/as-received extruded AM30 alloy. 


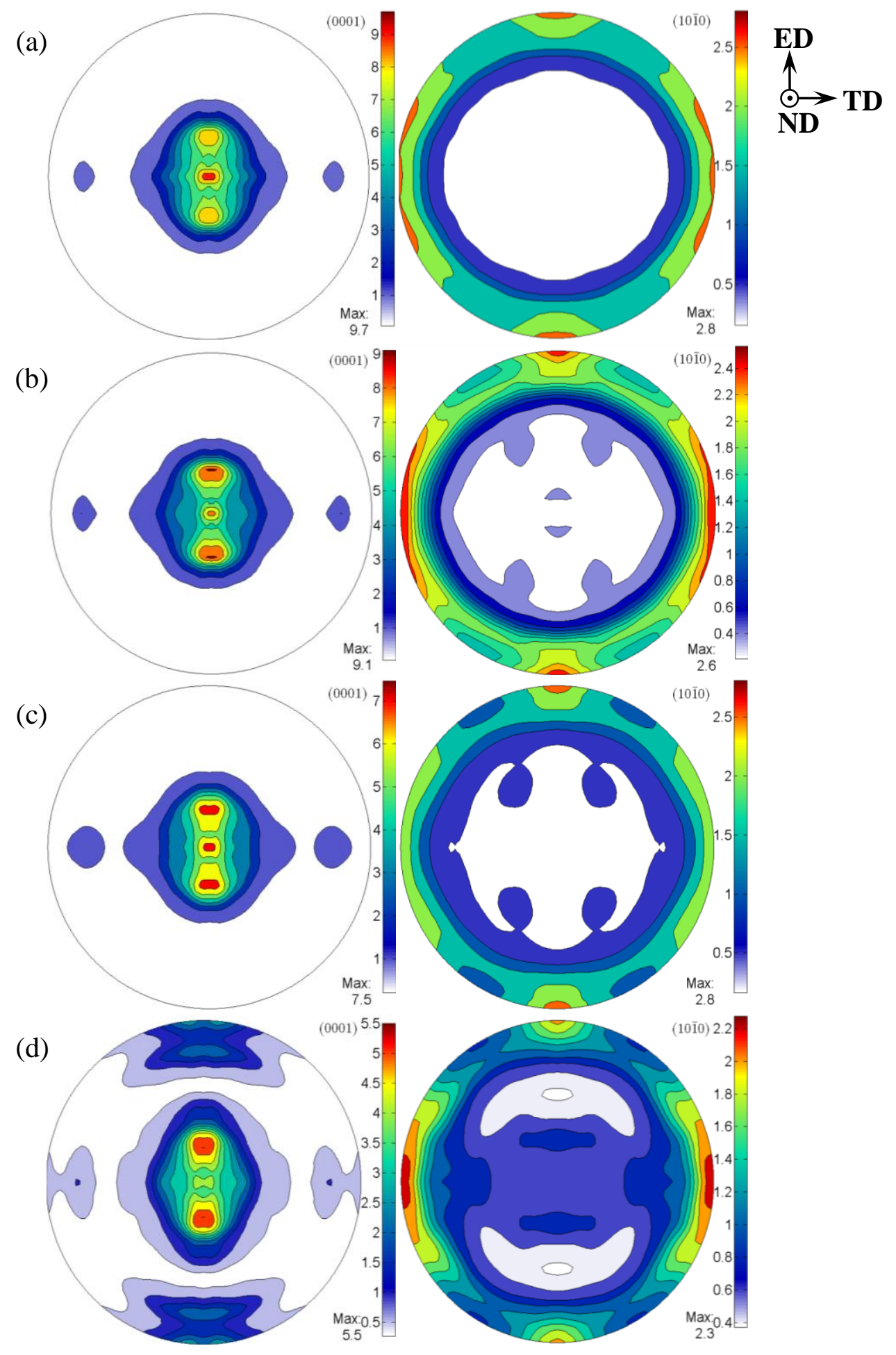

Figure 6.12: (0001) and (1010) pole figures of (a) 4.0\%ND, (b) $2.1 \% \mathrm{ED}-3.7 \% \mathrm{ND}$, (c) $3.7 \% \mathrm{ED}-$ $3.7 \% \mathrm{ND}$, (d) $7.9 \% \mathrm{ED}-3.7 \% \mathrm{ND}$. 
This indicated a somewhat intensifying basal texture during compression along the ND. In the two-step compressed sample of 2.1\%ED-3.7\%ND as shown in Fig. 6.12(b), the position of basal (0001) poles and (1010) poles were similar to those of the un-deformed sample (Fig. 4.1(b)) with an intensity of 9.1 MRD for the basal (0001) poles and 2.6 MRD for the prismatic (1010) poles. This corresponded well to the microstructure shown in Fig. 6.8(b) where nearly complete detwinning occurred and the initial twin-free microstructure was restored. At a higher pre-strain level, the two-step compressed 3.7\%ED-3.7\%ND sample in Fig. 6.12(c) also showed a texture position similar to the un-deformed sample with an intensity of 7.5 MRD for the (0001) basal poles and 2.8 MRD for the prismatic (1010) poles. This corresponded to some extent of detwinning with some grains containing thin/narrow twins, as shown in Fig. 6.8(d). As seen in Fig. 6.12(d) the same amount of re-compression along the ND but with a 7.9\% pre-strain along the ED led to a further weakening of texture with an intensity of 5.5 MRD for the (0001) basal poles and 2.3 MRD for the prismatic (1010) poles in comparison with Fig. 6.12(b) and (c). The weakening of the basal texture in this case was associated with some splitting of the (0001) basal poles towards the ED. As the twins encompassed the entire grain due to their growth during the 7.9\% pre-strain, the subsequent $3.7 \%$ re-compression along the ND was not sufficient to cause a complete de-twinning. Thus only partial de-twinning or twin shrinkage occurred with more remaining twins as shown in Fig. 6.8(f).

To quantify the change of texture during compression, the intensity of texture components $(\mathrm{f}(\mathrm{g}))$ as a function of cumulative true strain was evaluated and plotted in Fig. 6.13. The initial textures in the extruded AM30 alloy have been shown to consist of two sets of basal textures, i.e. 


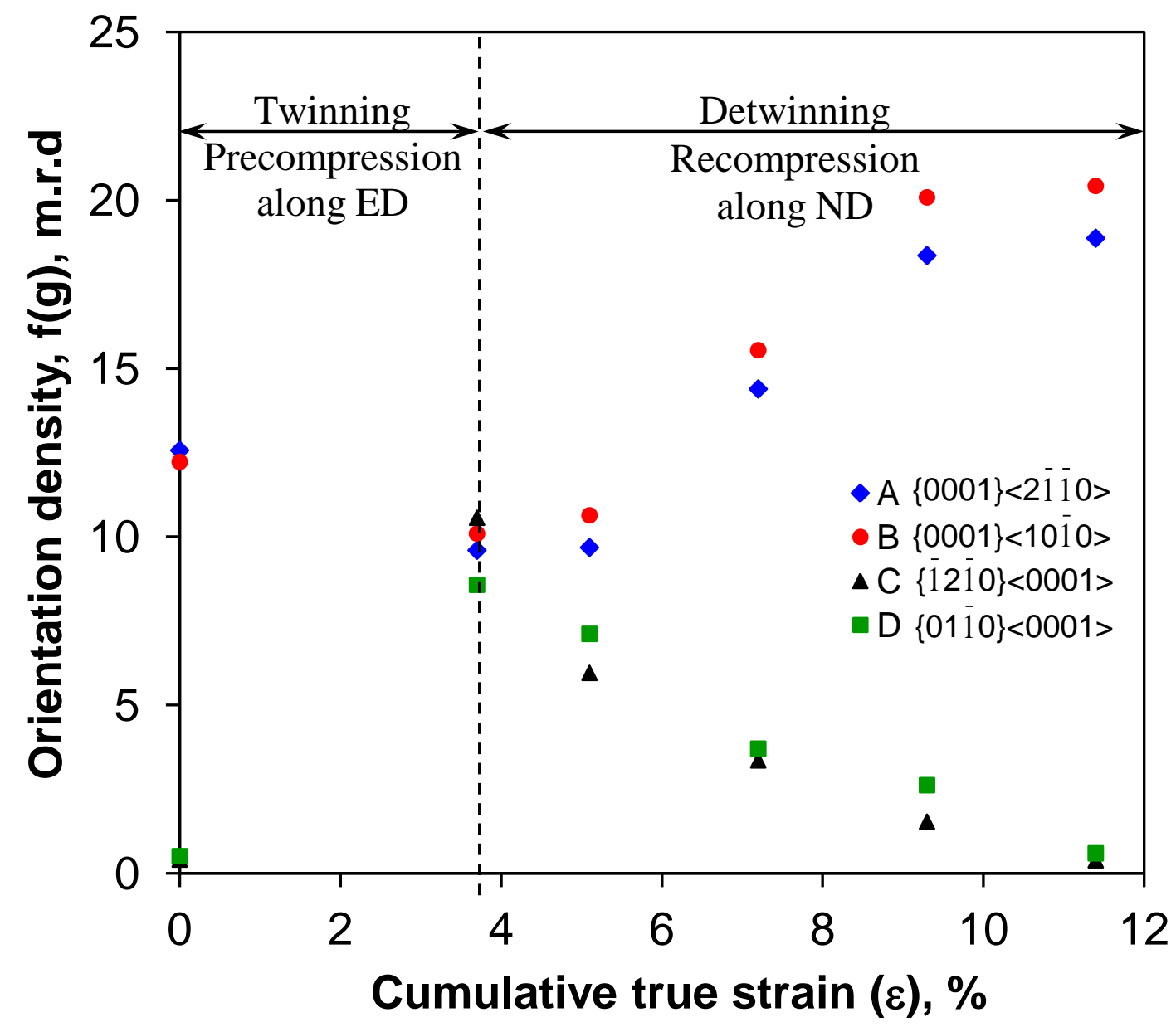

Figure 6.13: The change of intensity of main texture components with cumulative true strain.

$\mathrm{A}\{0001\}<2 \overline{1} \overline{1} 0>$ and $\mathrm{B}\{0001\}<10 \overline{10}$, which transformed into components $\mathrm{C}\{\overline{1} 2 \overline{1} 0\}<0001>$ and $\mathrm{D}\{01 \overline{10}<<0001>$, respectively, during compression along the ED as also presented in Fig. 4.8(b). During re-compression with increasing cumulative true strain along the ND, components A and B gradually intensified while components C and D faded away. This corresponds well to the microstructure shown in Fig. 6.9 which indicated de-twinning and the return of the texture back to the initial one (Fig. 4.1(b)). The microstructure after two-step compression of the 3.7\%ED-3.5\%ND (Fig. 6.9(c)) sample showed a reduction of twin width which was consistent with the result of the decreasing intensity of $\mathrm{C}$ and $\mathrm{D}$ components and increasing intensity of the 
initial A and B components (Fig. 6.13). As the complete de-twinning occurs due to increasing the re-compressive strain along the $\mathrm{ND}$, the $\mathrm{C}$ and $\mathrm{D}$ components vanished, which returned the texture to the initial components $\mathrm{A}$ and $\mathrm{B}$. The increasing intensity of $\mathrm{C}$ and $\mathrm{D}$ components after compression along the ED is related to the twin formation whereas intensification of $\mathrm{A}$ and $\mathrm{B}$ components during re-compression along the ND indicated de-twinning as shown in Figs 6.9 and 6.10 .

The phenomenon of de-twinning has been reported by a number of researchers $[83,169,181]$. For instance, Yu et al. [181] and Proust et al. [83] observed de-twinning in a magnesium single crystal and AZ31 magnesium alloy, respectively, under subsequent load reversal of precompressed samples. Also, when the loading direction was changed, grains that had previously twinned could de-twin $[97,169,177,182]$. These results were in agreement with the present observations that the $\{1012\}$ extension twins developed in the first step of compression along the ED, de-twinned in the second step of compression along the TD and ND (Figs. 6.7, 6.8(b), 6.8(d), 6.8(f), 6.9). The de-twinning may require a lower stress to be activated since the twins already existed, and no twin nucleation was necessary. In addition, back stresses generated by twin growth might aid the de-twinning process. To better understand this mechanism, a schematic diagram showing the process of twin formation, twin growth, de-twinning, and retwinning are illustrated in Fig. 6.14. A coordinate system is established with the Y-axis being parallel to the $c$-axis [0001] of an hcp unit cell in the magnesium matrix and the $\mathrm{X}$-axis oriented in the direction of [10 $\overline{10}$ ] which is positioned on the basal plane. 
(a)

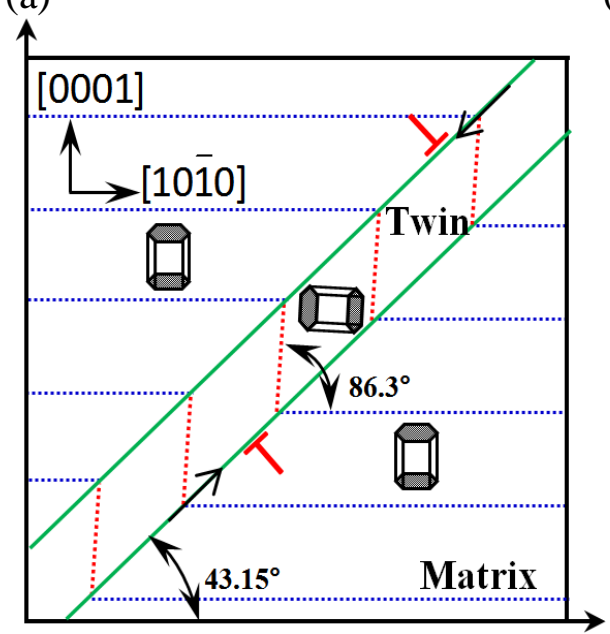

(c)

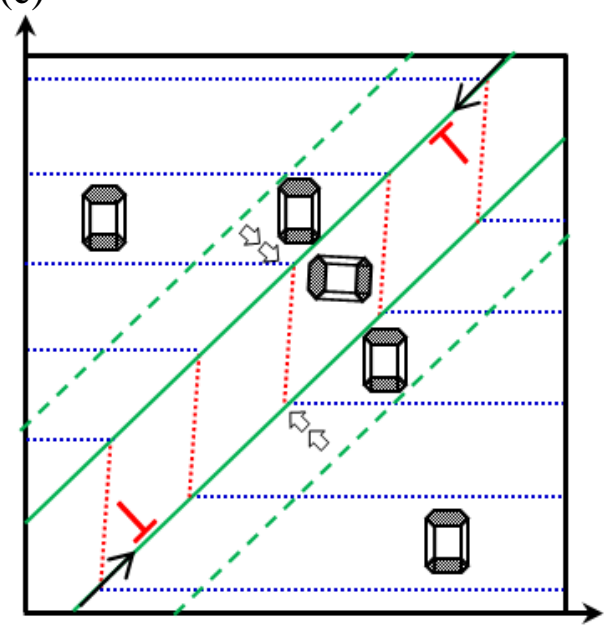

(b)

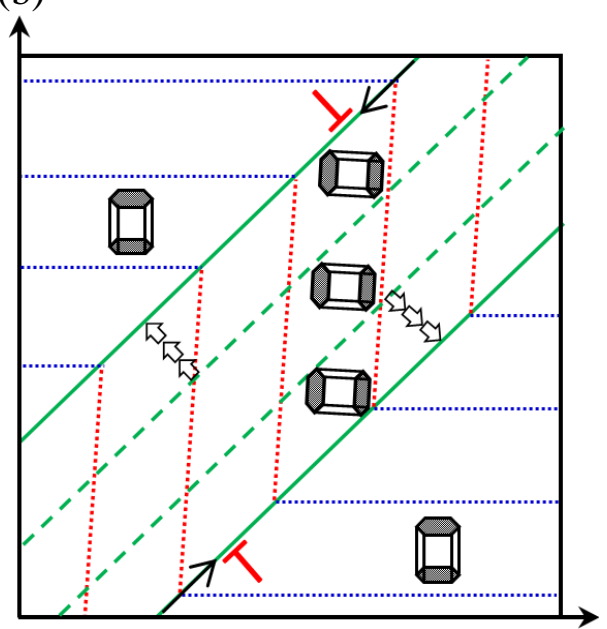

(d)

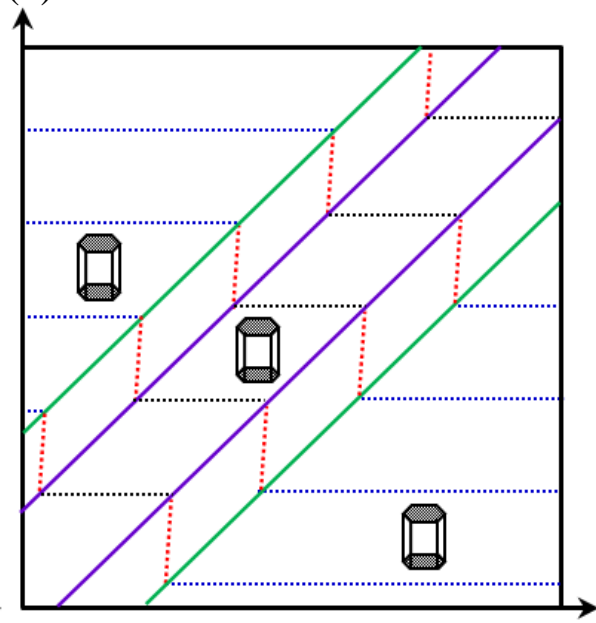

Figure 6.14: Schematic illustration of $\{1012\}$ extension twinning in a grain: (a) formation of a twin inside a grain by rotating an angle of $86.3^{\circ}$ of basal planes, (b) twin growth, through the nucleation and glide of twinning dislocations on the twin boundaries, (c) twin narrowing/shrinkage through the nucleation and glide of twinning dislocations with a reverse sign, (d) double (or secondary) twinning inside the pre-existing parent twin, with the same twin variant as the parent twin.

Consider a $\{10 \overline{1} 2\}$ extension twin within a grain, which is normally activated when a compressive stress is applied perpendicular to the [0001] $c$-axis or parallel to the (0001) basal 
plane (which is perpendicular to the paper or computer screen plane as indicated by the parallel dashed lines in Fig. 6.14(a). Twin-dislocation interactions play a key role in the twin growth. In the dislocation theory, upon meeting the $\{10 \overline{1} 2\}$ twin boundary, a slip dislocation can either penetrate into the twin and transmit to the other side of the twin boundary (TB), or undergo a core reconstruction, or dissociate into interfacial defects [121,169]. Twin growth occurs when the dissociation products are glissile twin dislocations (TDs) and glide through the TB in the matrix (Fig. 6.14(b)), and the driving force is the stress acting on TDs at the TB [121,169]. On the other hand, de-twinning, which is the opposite operation to twin growth, is accomplished by the gliding of TDs with an opposite sign in the twin along the TB (Fig. 6.14(c)), which begins when the resolved shear stress (RSS) is greater than the critical resolved shear stress (CRSS) for de-twinning. Depending on the shear stress the movement of TDs from the TB interface to the twinned region causes twin narrowing/shrinkage or de-twinning. Re-twinning (or double twinning) is also possible, which represents the activation of a twin within a twin. The double (or secondary) twin could have the same twin variant as or be different from the pre-existing parent twin variant [86]. If the active twin variants are identical, the double/secondary twin within twin band will have the basal planes parallel to those of the matrix (un-twinned region) (Fig. 6.14(d)). The volume occupied by this double twin is thus transferred from the original parent twin to the matrix, equivalent to de-twinning as well.

Finally, the effect of pre-strain on the compressive properties of the AM30 extruded magnesium alloy can be well correlated with the observed microstructural changes involving the texture evolution and twinning-de-twinning phenomena. As discussed above, in the ED-ED compressed samples, with the same loading direction in both the pre-compression and re-compression, twin 
formation and growth occurred with the applied strain in both steps. The extruded AM30 magnesium alloy selected in the present study contained a strong initial basal texture nearly perpendicular to the ED (Fig. 4.1(b)); as a result, the pre-compression along the ED caused profuse extension twinning (Fig. 6.5) and the stress-strain curve became sigmoidal in shape (Fig. 6.1). As mentioned previously, the formation of extension twins caused a rotation of $c$-axes of grains by an angle of $86.3^{\circ}$; and with increasing pre-strain more and more grains twinned. This change eventually caused the rotation of the $c$-axes of most grains towards the compression direction [129], as seen from Fig. 4.7 (b,c) vs. Fig. 4.1(b). It is clear that during the recompression of the samples having already been subjected to a large amount of pre-strain (e.g., $7.5 \%$ or $8.2 \%$ ), the $c$-axes of most grains were oriented unfavorably for the further twin formation, as also demonstrated by the disappearance of the sigmoidal shape in the stress-strain curves (Fig. 6.2(a)). This indeed resulted in a so-called hard orientation for further twinning, and a higher stress was required to initiate the slip of dislocations in the twinned material [63], thus increasing the compressive yield stress of the pre-strained samples during re-compression (Fig. 6.3(a)). In this circumstance, deformation would be dominated by the dislocation slip, and the exhaustion of deformation would cause a lower UCS (Fig. 6.3(b)) and hardening capacity (Fig. 6.3(c)) based on Eq. (6.3) due to the premature failure. A similar effect of pre-strain on the YS and UCS in a hot-rolled AZ31 magnesium alloy has also been observed by Xin et al. [20], where the $\{10 \overline{1} 2\}$ extension twins formed by pre-compression along the rolling direction resulted in grain refinement which dramatically enhanced both the YS and UCS of reloading along the transverse direction. 
In the ED-TD compressed samples, as mentioned above, two seemingly opposite phenomena of new twin formation and de-twinning were co-existent, depending on the orientation of grains (un-twinned regions) and twins developed in the first step (along the ED) with respect to the loading direction of TD in the second step. This was also related to the presence of multiple sets of textures after the first step pre-strain along the ED (Fig. 4.7(b)). The formation of new twins in the second step along the TD (Fig. 6.6(b)) led to grain sub-divisions, somewhat equivalent to grain refinement, which would thus increase the YS, and UCS (Fig. 6.3(a) and (b)) based on the well-known Hall-Petch relationship. This is also corroborated by the observations reported by Oppedal et al. [183], where the authors have attributed the dramatic stress increase in a strongly textured hcp metal after loading along an orientation causing profuse twinning to a combined effect of the Hall-Petch mechanism related to grain refinement by twinning, with the twinninginduced re-orientation requiring activation of hard slip modes. On the other hand, the occurrence of de-twinning (Fig. 6.7) made the pre-existent twins narrower and shorter, and drove the $c$-axes of hcp unit cells in some grains back to the as-extruded state (Fig. 6.11(b) vs. Fig. 4.1(b)). The reversal of textures would help get rid of the cold-work effect arising from the pre-strain by lessening the hard orientations of further deformation, thus recovering the fracture strain to be equivalent to that of the as-extruded AM30 magnesium alloy (Fig. 6.2).

Due to the re-orientation of the $c$-axes towards the ED after pre-strain along the ED, the $c$-axes would be perpendicular to the compression axis in the subsequent re-compression along the ND. It follows that the twinned region would be preferentially oriented to twin again (i.e., twin back or de-twin) which is evident from the appearance of the sigmoidal shape in the stress-strain curves (Fig. 6.2(c)). This resulted in a favorable orientation for further loading, and a lower 
stress required for de-twinning in the twinned material [102], thus decreasing the compressive yield stress of the pre-strained samples during re-compression (Fig. 6.2(c)). At higher pre-strain levels, grains are heavily twinned and a relatively lower re-compression strain along the ND makes de-twinning harder, resulting in incomplete de-twinning as shown in Fig. 6.8(f). In other words, the degree of twinning during the pre-strain along the ED is larger than that of detwinning (or twin shrinkage) during the subsequent re-compression along the ND. In addition, the remaining thin/narrow twins in the second step along the ND (Fig. 6.8(d) and (f)) led to grain sub-divisions, somewhat equivalent to grain refinement, which resulted in an increase in the UCS to a certain extent (Fig. 6.3(b). The reversal of the texture (Fig. 6.12(d) and (e)) after recompression along the ND is a strong indication of de-twinning.

\subsection{Summary}

This chapter described the effect of pre-compressive deformation on microstructure and texture development. The results focused on the influence of twinning introduced by first-step compression along the extrusion direction (ED) on the subsequent twinning, de-twinning, texture development and strain hardening using three different paths, i.e., extrusion direction (ED-ED), transverse direction (ED-TD) and normal direction (ED-ND) during the second step compression.

The compressive loading in both the ED and TD led to a similar sigmoidal-shaped true stresstrue strain behavior in the as-extruded AM30 alloy due to the presence of two sets of basal textures with the $c$-axes aligned almost parallel to the ND, which facilitated the occurrence of the 
$\{1012\}$ extension twinning, whereas the compressive loading directly in the ND showed a slipdominated flow curve.

The compressive properties were significantly dependent on both the pre-strain amount and the re-loading direction. In the case of ED-ED compressed samples, with increasing pre-strain the compressive yield strength (YS) linearly increased, while the ultimate compressive strength (UCS) and hardening capacity linearly decreased. The change range of strain hardening rate in stage $\mathrm{B}\left(\Delta \theta_{\mathrm{B}}\right)$ remained nearly constant at a lower pre-strain level up to $\sim 2.3 \%$, then decreased linearly with increasing pre-strain. In the case of the ED-TD compressed samples, with increasing pre-strain up to $7.5 \%$ the sigmoidal shape of the flow curves and the related stage B hardening still remained, despite a lesser extent. In this case both the YS and UCS increased, and the hardening capacity and $\Delta \theta_{\mathrm{B}}$ value decreased in a non-linear manner. In the ED-ND compressed samples, the reloading true stress-true strain curves displayed a sigmoidal shape that was associated with the occurrence of re-twinning (i.e., back-twinning or de-twinning). With increasing pre-strain along the ED, the compressive yield strength (YS) in the ND decreased, while the ultimate compressive strength (UCS) increased. In the absence of pre-strain the compression in the ND led to conventional stage III hardening followed by stage IV hardening. At the lower pre-strain levels of $2.1 \%$ and $3.7 \%$, two hardening stages of $\mathrm{B}$ and $\mathrm{C}$ were present, while three distinct hardening stages of $\mathrm{A}, \mathrm{B}$ and $\mathrm{C}$ occurred at the higher pre-strain levels of $5.4 \%$ and $7.9 \%$. The peak value between stages $\mathrm{B}$ and $\mathrm{C}$ and the slope of strain hardening rate in stage B linearly decreased with increasing pre-strain. 
In the ED-ED compressed samples, after the two steps of compression in the same direction the disappearance of some twin boundaries or the coalescence of twins via twin growth was observed. However, the extent of twin growth in the two-step compression was lower than that in the one-step continuous compression at an equivalent total strain level, as the twin boundaries were more visible and the intensity of cumulative textures after the $c$-axes rotating towards the compression direction was lower in the two-step compression, in comparison with the one-step continuous compression. In the ED-TD compressed samples, a $4.0 \%$ re-compression along the TD after a $4.0 \%$ pre-strain along the ED led to the co-existence of two seemingly opposite phenomena, i.e., the formation of new secondary twins and de-twinning due to the presence of multiple sets of textures after the first-step pre-straining, depending on the orientation of grains (or un-twinned regions) and twins developed in the first step (along the ED) with respect to the second-step loading direction of TD. The occurrence of twin narrowing/shortening or detwinning caused by such a strain path change in the second-step compression was verified by both the surface microstructural examination and the reversal of textures. In the subsequent second-step compression along the ND, back-twinning (de-twinning) occurred, which was verified by both the microstructural examination and the reversal of textures as well. The detwinning activity was observed to decrease with increasing pre-strain.

The texture measurements revealed that the $c$-axes of hcp unit cells were always rotated towards the compression direction, regardless of the direct compression in the ED, TD or ND. Texture weakening was achieved via the pre-compression in the ED and subsequent re-compression along the TD and ND. With increasing pre-strain in the ED, while keeping the re-compression 
amount in the ND constant (3.7\%), the extent of texture weakening increased. Further studies are needed to identify an optimal compression condition to arrive at potential texture randomization. 


\section{CHAPTER 7}

\section{Influence of Pre-Deformation and Subsequent Annealing on Strain Hardening and Anisotropy}

\subsection{Introduction}

Magnesium alloys normally form a strong texture during manufacturing processes and exhibit low room temperature formability and directional anisotropy due to the polar characteristics of deformation twinning, which seriously restrict their structural application [184,185]. Thermomechanical processing could produce new microstructures which could alter the detrimental strong basal texture and thus improve formability of wrought magnesium alloys $[105,113,150,175]$.

To improve formability, recrystallization is an effective approach which involves the formation of a new grain structure in a plastically deformed material by the formation and migration of high angle grain boundaries driven by the stored energy of deformation [186-189]. The recrystallization process of plastically deformed materials serves to soften the material and restore the ductility and formability. As recrystallization needs nucleation sites in deformed structures, deformation twins could offer the possibility to form recrystallized grains with modified size, shape or texture, especially for the magnesium alloys without second-phase precipitates [187]. In the commercial production of wrought magnesium alloys, dynamic recrystallization in various forming processes has been studied in addition to the detailed investigation of static recrystallization [36,105,108,141,190-195]. For example, the refinement of 
grains in alloys and the ensuing improvement in the mechanical properties can be achieved through a variety of processing techniques such as equal channel angular extrusion, rolling, and multi-directional forging [30,63,196-203].

The study pertaining to the static recrystallization of cold deformed magnesium alloys has been limited, in spite of numerous reports on other materials such as steels and aluminum alloys. In deformed magnesium alloys at room temperature, the mechanical properties are basically enhanced due to the strain hardening effect arising from internal energy accumulation, which would be diminished by recovery and recrystallization in the subsequent annealing [204-206]. Levinson et al. [113] reported the microstructure and texture evolution during isothermal static annealing of an AZ31magnesium alloy in the cast and extruded states to evaluate the contribution of extension and contraction twins to the recrystallized microstructure after room temperature compression to two strains of $5 \%$ and $15 \%$ and then subjected to annealing at $175^{\circ} \mathrm{C}$ and $275^{\circ} \mathrm{C}$. Their results showed that contraction and double twins were potent sites for recrystallized nuclei and produced a distribution of new orientations, while no significantly influence on the final texture was observed. Yang et al. [114] also studied the effect of prior strain on static recrystallization of a hot-deformed AZ31 magnesium alloy during isothermal annealing at temperatures of $220^{\circ} \mathrm{C}, 230^{\circ} \mathrm{C}$ and $240^{\circ} \mathrm{C}$. It was observed that ultrafine grains were evolved by grain fragmentation due to continuous dynamic recrystallization during hot deformation, and grain coarsening occurred during the subsequent annealing together with a significant change in the deformation texture. 
However, no such studies are seen in the open literature on the recrystallization during annealing of AM30 magnesium alloy, after pre-compression at room temperature. It is unclear how the strain hardening rate and texture change occurred in the pre-deformed and annealed alloy during re-compression at room temperature. The objectives of this study were, therefore, to characterize the recrystallization behavior of the pre-compressed AM30 magnesium alloy and evaluate the changes in microstructure and texture during re-compression of annealed samples along with the strain hardening behavior.

\subsection{Effect of Pre-Strain and Subsequent Annealing on Compressive Properties}

The true stress-true strain curves of the AM30 extruded magnesium alloy without pre-strain and with a pre-strain of $4.3 \%$ along the ED are shown in Fig. 7.1. It is seen that the compressive yield stress increased from $\sim 91 \mathrm{MPa}$ to $\sim 137 \mathrm{MPa}$ after the application of a $4.3 \%$ pre-strain. However, the ultimate compressive strength decreased from $\sim 325 \mathrm{MPa}$ to $\sim 315 \mathrm{MPa}$ together with a lower fracture strain. Also, both curves showed a skewed/sigmoidal shape, while the extent of the skewness reduced in the pre-strained samples. Because of the presence of strong basal texture in the as-extruded alloy with the $c$-axes of most grains aligned almost parallel to the ND (Fig. 4.1(b)), during compression along the ED the samples were subjected to a compressive loading perpendicular to the $c$-axes $[98,129,169]$. Such a loading condition facilitated the occurrence of $\{1012\}$ extension twinning that re-oriented the basal planes or $c$-axes by $86.3^{\circ}$, which made a harder orientation for further twin formation and thus increased the yield stress after pre-strain (Fig. 7.1). Details about the effect of pre-strain on the yield stress and ultimate compressive strength have been presented in [169]. 


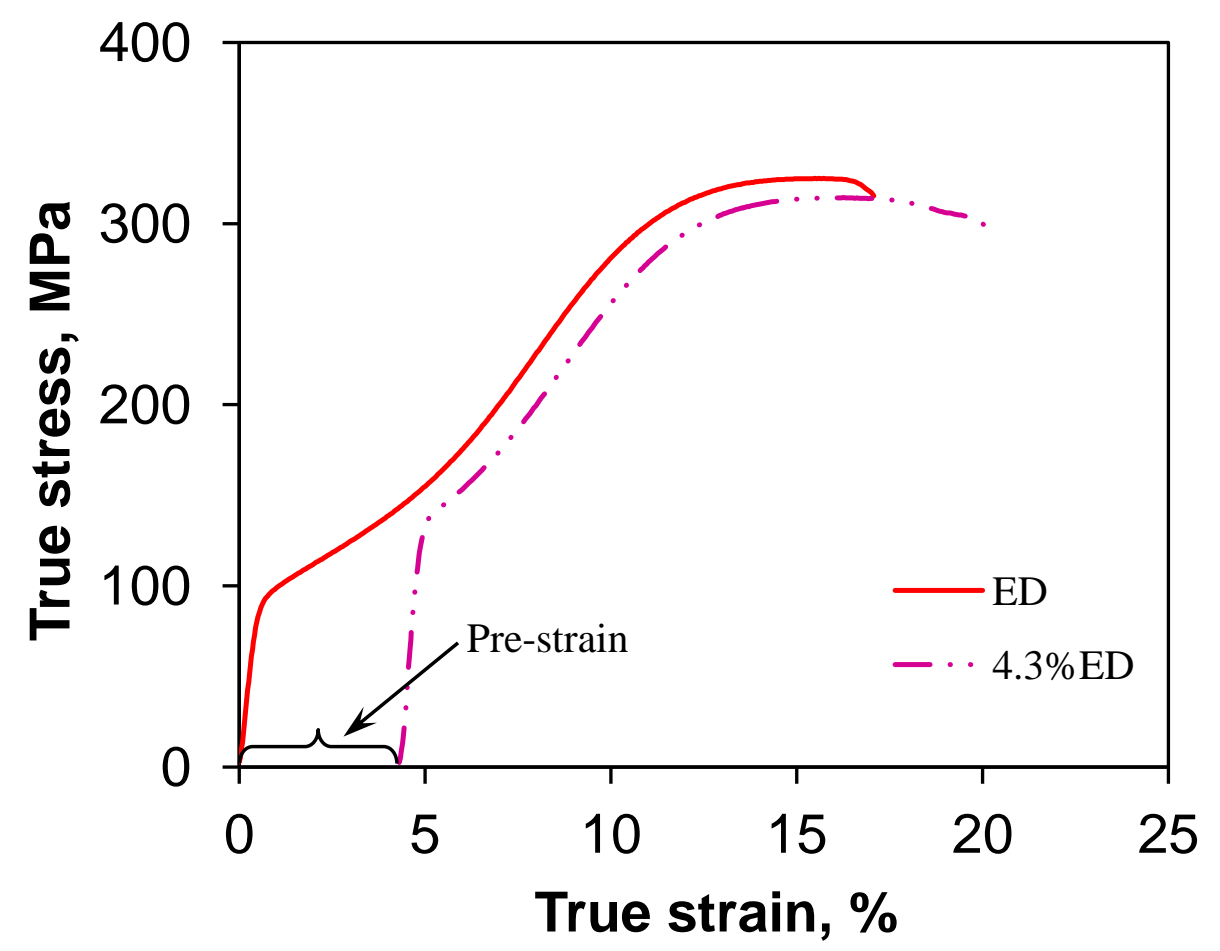

Figure 7.1: Typical true stress-true strain curves of extruded AM30 with and without a 4.3\% pre-strain along the ED.

The true stress-true strain curves of the pre-strained samples that were subjected to annealing at different temperatures are shown in Fig. 7.2(a), where the re-compression was also conducted along the ED, as symbolized by the $4.3 \% \mathrm{ED}$-annealing temperature-annealing time in the graph. It is of interest to see that a significant change in the yield stress and the shape of true stress-true strain curves occurred after annealing in comparison with Fig. 7.1. The evaluated results were plotted in Fig. 7.2(b) and (c). Increasing the annealing temperature to $250^{\circ} \mathrm{C}, 350^{\circ} \mathrm{C}$, and $450^{\circ} \mathrm{C}$ while keeping a constant annealing time of $3 \mathrm{~h}$ led to an almost linear decrease in the compressive yield stress (CYS) from $\sim 137 \mathrm{MPa}$ (for the pre-strained but non-annealed sample) to $\sim 101 \mathrm{MPa}$, $\sim 96 \mathrm{MPa}$, and $\sim 77 \mathrm{MPa}$ (Fig. 7.2(b)), respectively. 

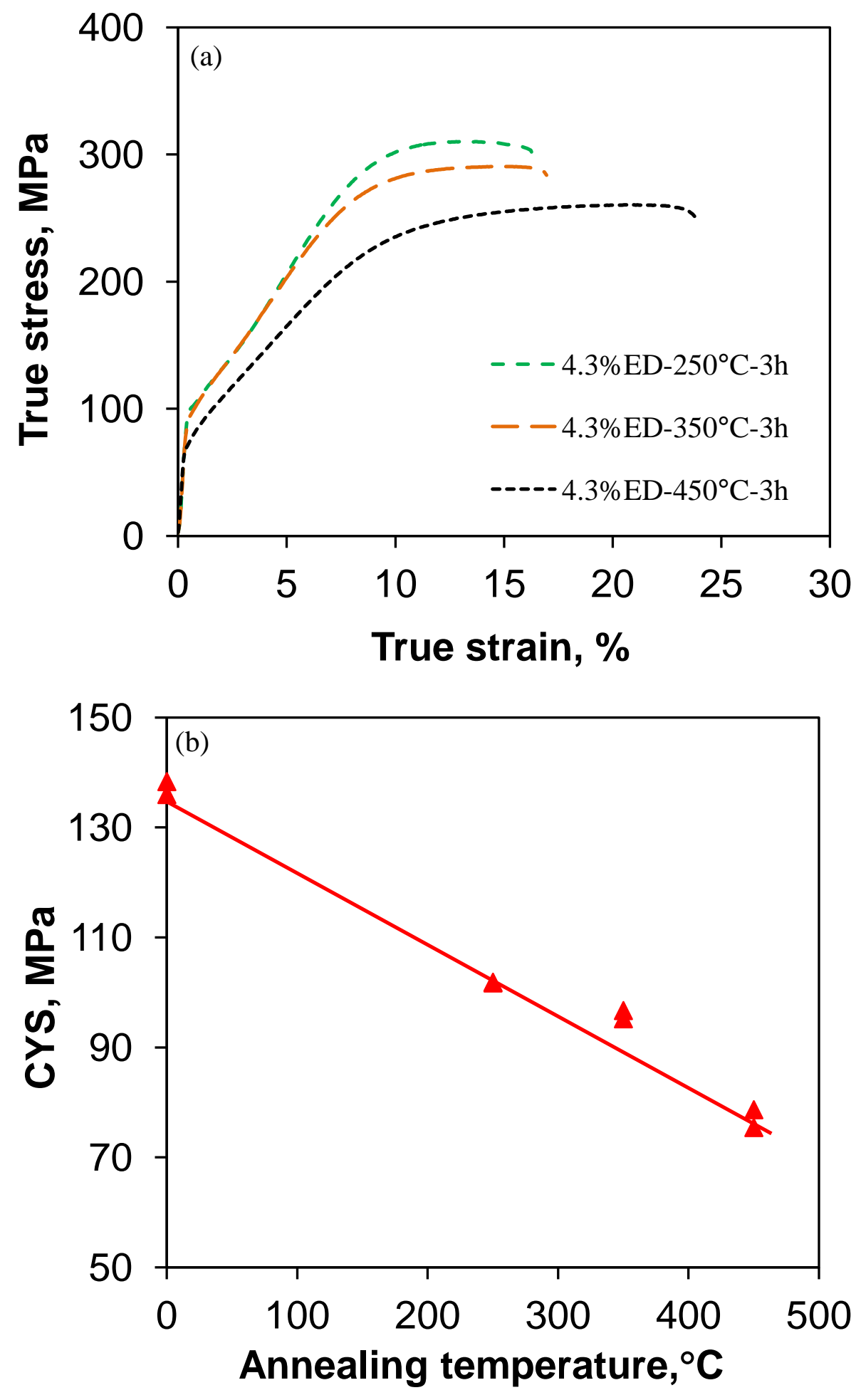


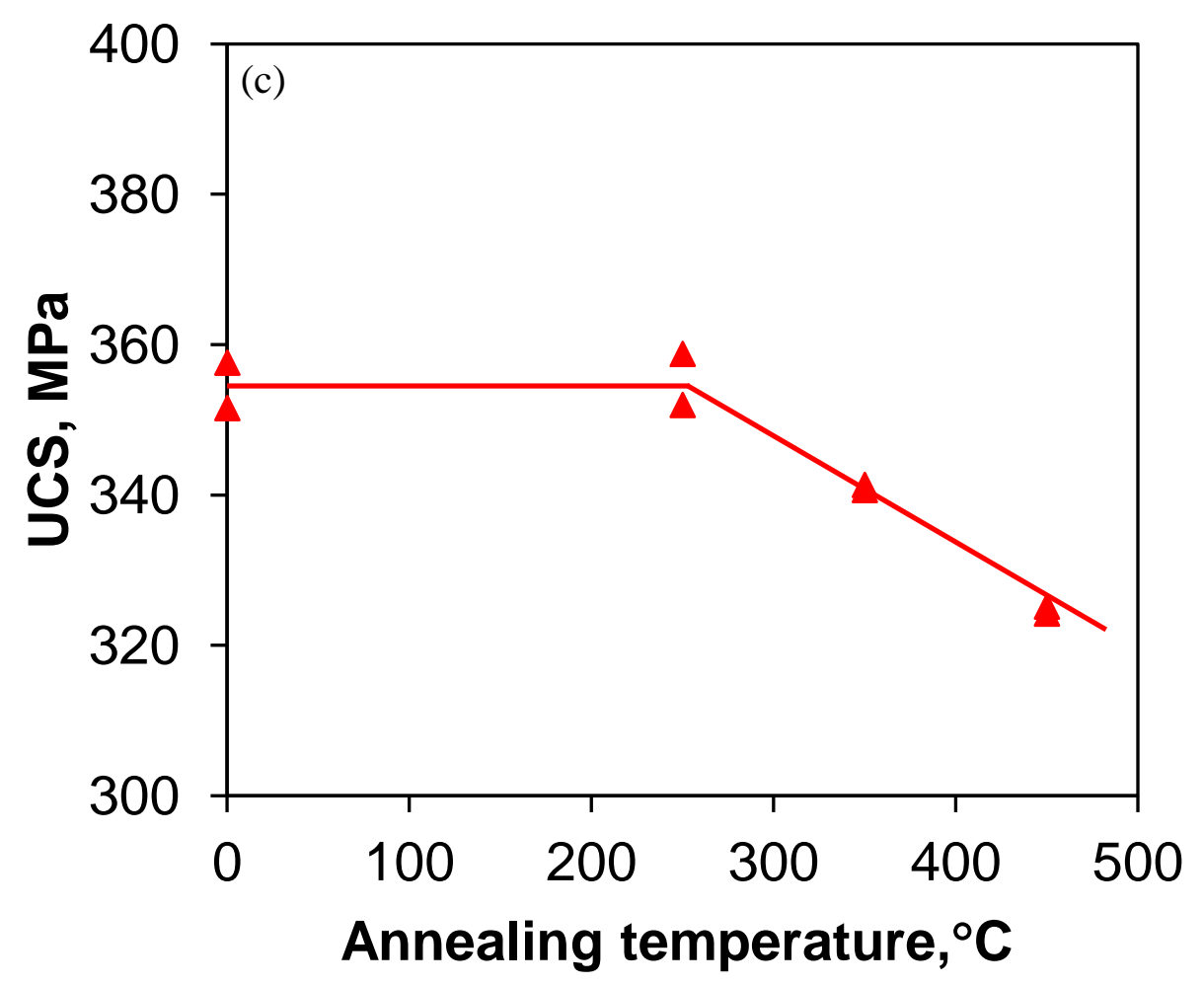

Figure 7.2: (a) Typical true stress-true strain curves of $4.3 \%$ pre-strained and annealed samples at $250^{\circ} \mathrm{C}, 350^{\circ} \mathrm{C}$ and $450^{\circ} \mathrm{C}$ for $3 \mathrm{~h}$, (b) compressive yield strength (CYS), and (c) ultimate compressive strength (UCS) as a function of annealing temperature.

However, the ultimate compressive strength (UCS) remained unchanged (i.e., 355 MPa) when the annealing was conducted at $250^{\circ} \mathrm{C}$, then it decreased linearly to $341 \mathrm{MPa}$, and $325 \mathrm{MPa}$ with increasing annealing temperature to $350^{\circ} \mathrm{C}$ and $450^{\circ} \mathrm{C}$ (Fig. $7.2(\mathrm{c})$ ). Also, the fracture strain increased and the extent of skewness decreased as the annealing temperature increased (Fig. 7.2(a)). On the other hand, at a given annealing temperature of $450^{\circ} \mathrm{C}$, only a weak effect of annealing time on the true stress-true strain curves was observed, as shown in Fig. 7.3. Specially, the compressive yield stress was nearly the same, while a slight decrease in the ultimate compressive strength with increasing annealing time was observed. It is clear that the effect of 
annealing temperature was more significant than that of annealing time. These changes in the compressive deformation characteristics were attributed to the change in the microstructure and texture which will be discussed later. The change in the compressive stress-strain behavior could indeed be better seen from the strain hardening rate $d \sigma / d \varepsilon$ (where $\sigma$ is the true stress and $\varepsilon$ is the true strain) as a function of true strain in the pre-strained and annealed samples at different temperatures and times, as shown in Fig. 7.4(a) and (b). The re-compression of the samples subjected to a pre-strain of $4.3 \%$ showed clearly three distinct stages of strain hardening, which are also illustrated schematically in the inset of Fig. 7.4(a).

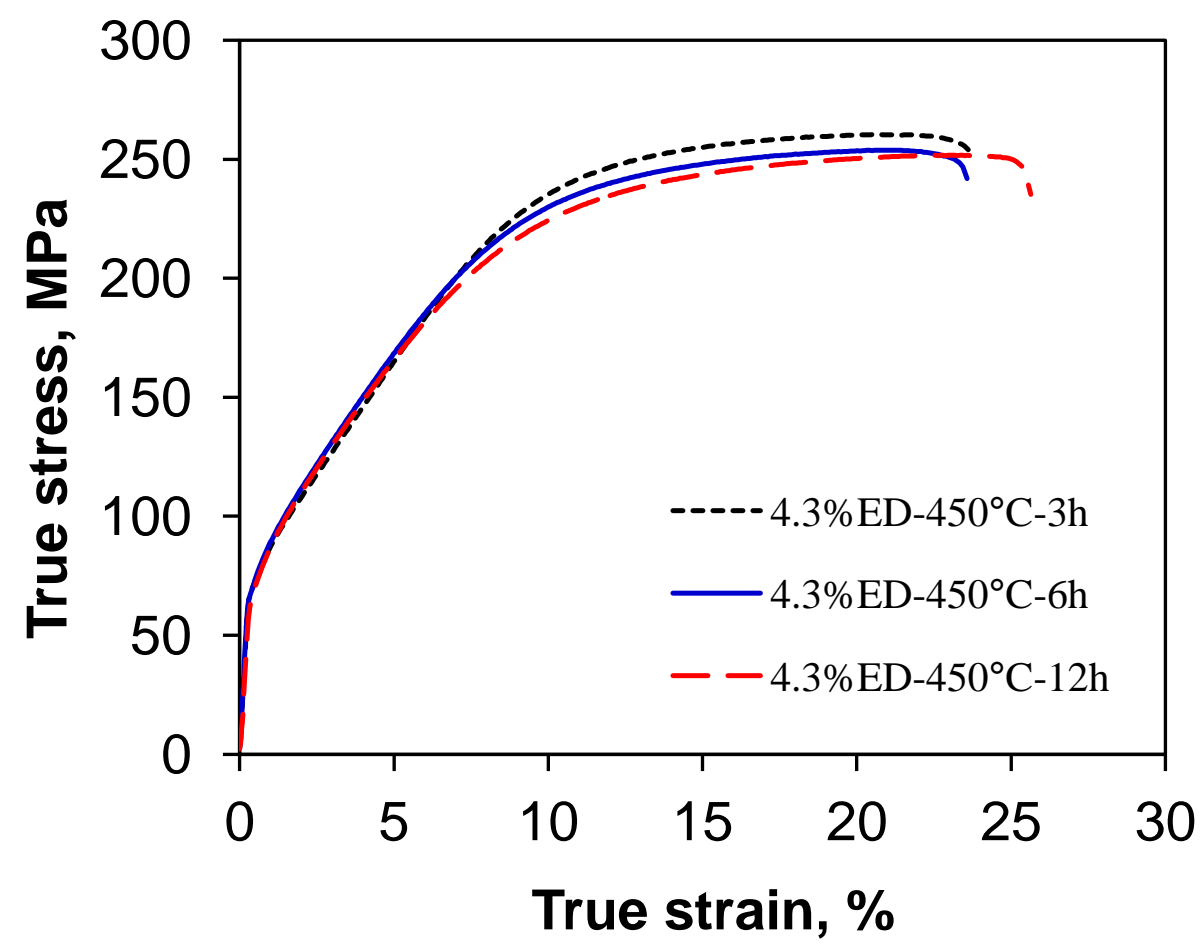

Figure 7.3: Typical true stress-true strain curves of $4.3 \%$ pre-strained and annealed samples at $450^{\circ} \mathrm{C}$ for different times of $3 \mathrm{~h}, 6 \mathrm{~h}$ and $12 \mathrm{~h}$. 
This was similar to the strain hardening characteristics of the extruded AM30 alloy $[135,169]$. Stage A was characterized by a rapidly falling strain hardening rate, followed by stage B with an increasing strain hardening rate, and then stage $\mathrm{C}$ with a decreasing strain hardening rate again until failure. With increasing annealing temperature while keeping an annealing time of $3 \mathrm{~h}$, stage B became gradually weaker (Fig. 7.4(a)). As seen from Fig. 7.4(b), while a pronounced change occurred after annealing at $450^{\circ} \mathrm{C}$, in comparison with the $d \sigma / d \varepsilon$ vs. $\varepsilon$ curve of the sample that was just subjected to pre-strain without annealing, the change of these curves affected by the annealing time from $3 \mathrm{~h}$ to $12 \mathrm{~h}$ at $450^{\circ} \mathrm{C}$ was relatively small. This corresponded to the true stress-true strain curves shown in Fig. 7.3. It is seen from Fig. 7.4(a) and (b) that stage C could be approximated by a linear decrease with increasing true strain, which indeed reflected stage III hardening in the tensile deformation [168]. Also, stage C was observed to move slightly downward with increasing annealing time (Fig. 7.4(b)).

As mentioned above, since stage B reflects a unique strain hardening characteristic in the compressive deformation of magnesium alloys, more evaluations were thus done here, aiming to understand better the effect of annealing temperature and time. These evaluations are (1) the magnitude or range of stage $\mathrm{B}$ (denoted as $\Delta \theta_{\mathrm{B}}$ ), defined as the peak $d \sigma / d \varepsilon$ value in-between stages $\mathrm{B}$ and $\mathrm{C}$ minus the valley $d \sigma / d \varepsilon$ value in-between stages $\mathrm{A}$ and $\mathrm{B}$, as schematically indicated in the inset of Fig. 7.4(a), and (2) the slope of stage B. The evaluated values are plotted in Fig. 7.5. It is seen from Fig. 7.5(a) that the magnitude of stage B decreased with increasing annealing temperature from $250^{\circ} \mathrm{C}$ to $450^{\circ} \mathrm{C}$. It is also clear that the slope of stage $\mathrm{B}$ decreased with increasing annealing temperature and time, as seen in Fig. 7.5(b) and (c), respectively. 

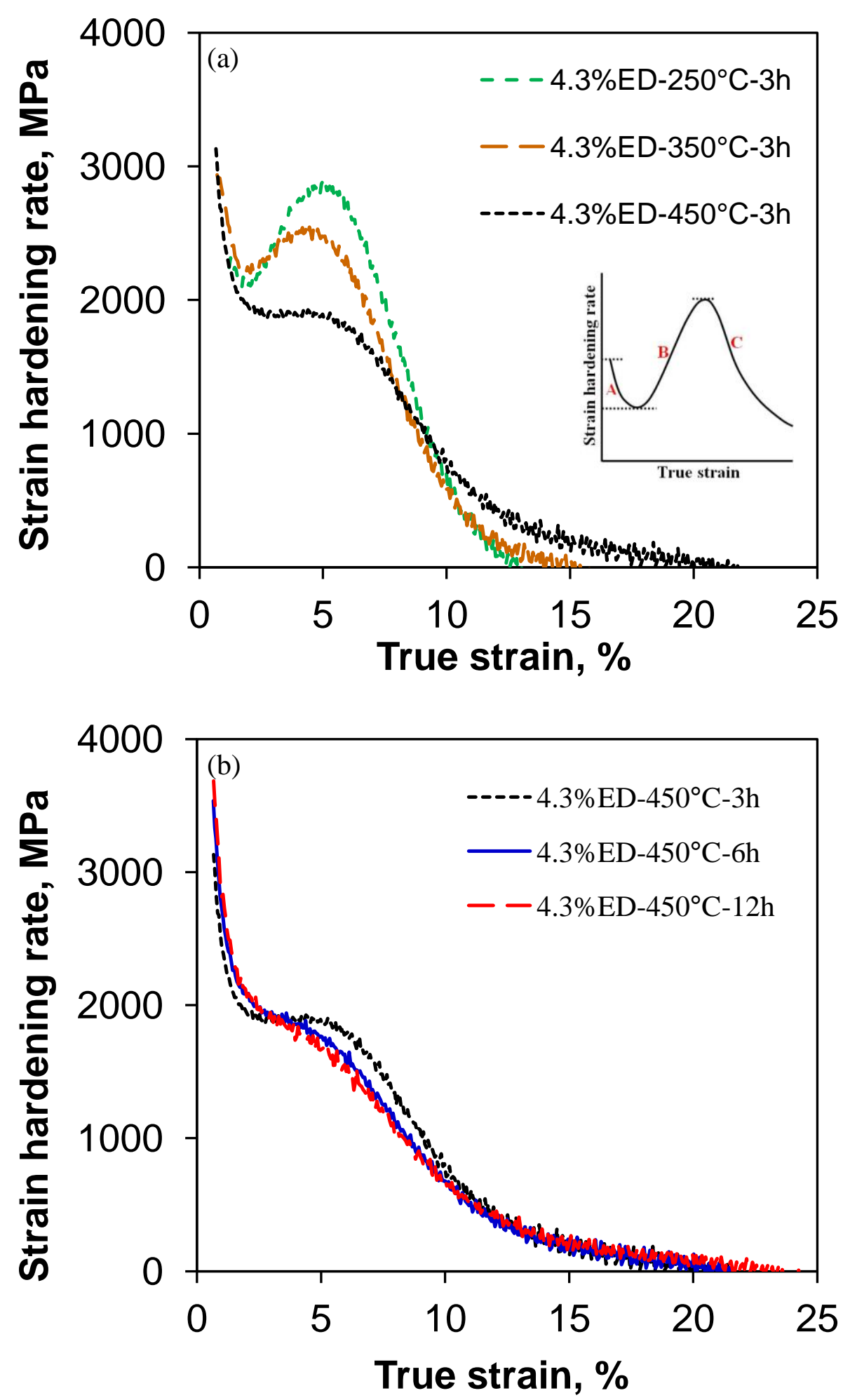

Figure 7.4: Strain hardening rate vs. true strain of $4.3 \%$ pre-strained and annealed AM 30 alloy at (a) $250^{\circ} \mathrm{C}, 350^{\circ} \mathrm{C}$ and $450^{\circ} \mathrm{C}$ for $3 \mathrm{~h}$, (b) $450^{\circ} \mathrm{C}$ for $3 \mathrm{~h}, 6 \mathrm{~h}$ and $12 \mathrm{~h}$. 

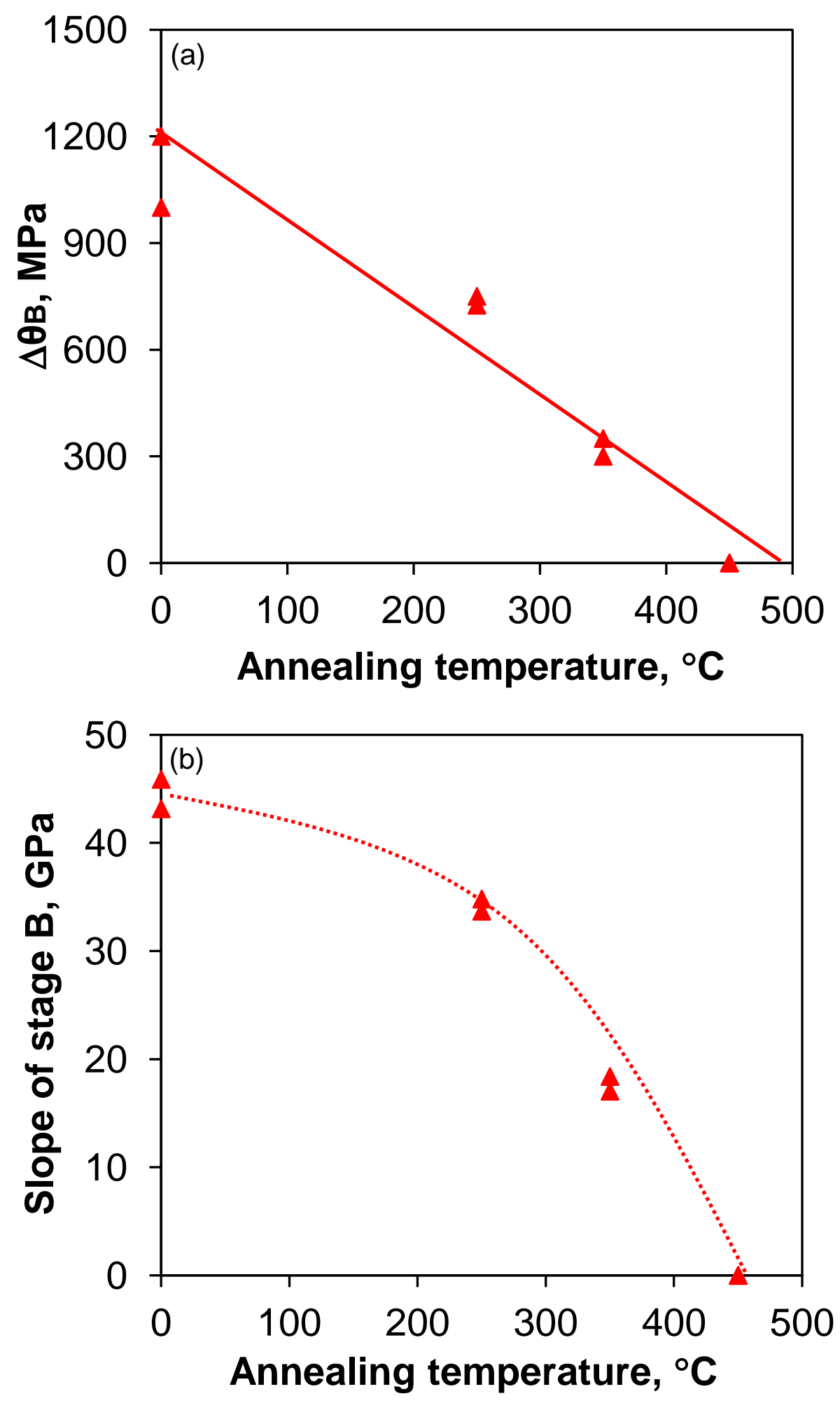


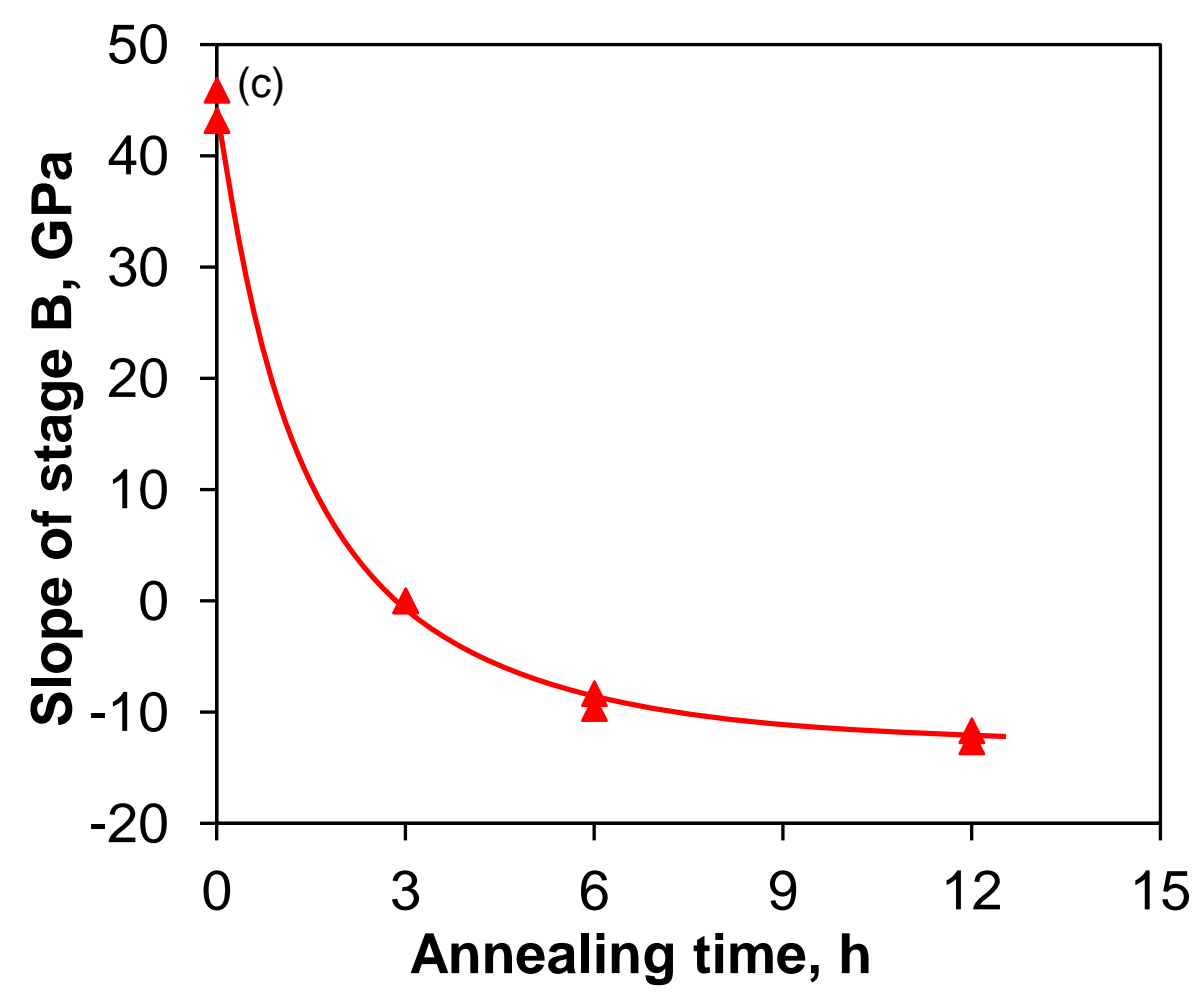

Figure 7.5: Stage B hardening behavior of 4.3\% pre-compressed and annealed AM30 Mg alloy, (a) the extent of stage $\mathrm{B}\left(\Delta \theta_{\mathrm{B}}\right)$ and (b) the slope of stage $\mathrm{B}$ as a function of annealing temperature while keeping a constant annealing time of $3 \mathrm{~h}$, (c) the slope of stage B as a function of annealing time while keeping a given annealing temperature of $450^{\circ} \mathrm{C}$.

However, the decrease of the slope was in the convex form with increasing annealing temperature up to $450^{\circ} \mathrm{C}$ (Fig. $7.5(\mathrm{~b})$ ) and in the concave form with increasing annealing time up to $12 \mathrm{~h}$ (Fig. $7.5(\mathrm{c})$ ). This suggests a more significant influence of annealing temperature than annealing time on the slope of stage B. Indeed, a slope of almost zero was obtained at $450^{\circ} \mathrm{C}$ for 3h (Fig. 7.5(a)), which corresponded to the nearly flat portion on the $d \sigma / d \varepsilon$ vs. $\varepsilon$ curve in stage B (Fig. 7.4). Increasing the annealing time from $3 \mathrm{~h}$ to $6 \mathrm{~h}$ and $12 \mathrm{~h}$ at $450^{\circ} \mathrm{C}$ led to further weakening of stage B, as indicated by gradually more negative slope (Fig. 7.5(c)). Stage B is 
anticipated to fade away either at a further higher annealing temperature or longer annealing time when the negative slope of stage B reaches that of stage C. Further studies in this aspect are needed. A similar decreasing trend in strain hardening was observed during multi-directional forging of an AZ31 magnesium alloy at room temperature with increasing strain level [207]. This type of change in the hardening behavior corresponded to microstructure and texture evolution, which will be presented in the next sections.

\subsection{Effect of Pre-Strain and Annealing on Microstructure Development}

Microstructure evolution was examined via optical microscopy for the $4.3 \%$ pre-strained samples after annealing at $450^{\circ} \mathrm{C}$ for different times. Prior to conducting annealing, at this amount of prestrain most grains were subjected to $\{1012\}$ extension twinning which traversed the parent grains and interacted with each other, as reported in [169]. The volume fraction of extension twins was measured after annealing for $0.25 \mathrm{~h}, 1 \mathrm{~h}, 3 \mathrm{~h}, 6 \mathrm{~h}, 12 \mathrm{~h}$, and $24 \mathrm{~h}$ which was plotted as a function of annealing time in Fig. 7.6, in conjunction with some typical images showing the visual change of twins. It is clear that the volume fraction of twins decreased with increasing annealing time, which could be expressed by the following equation,

$$
V_{f}=\beta t^{\alpha}
$$

where $V_{f}$ is the volume fraction of twins, $t$ is the annealing time in sec, $\alpha$ and $\beta$ are two empirical material constants ( $\alpha=-0.95$ and $\beta=390.2$ obtained for the AM30 alloy in the present study). 


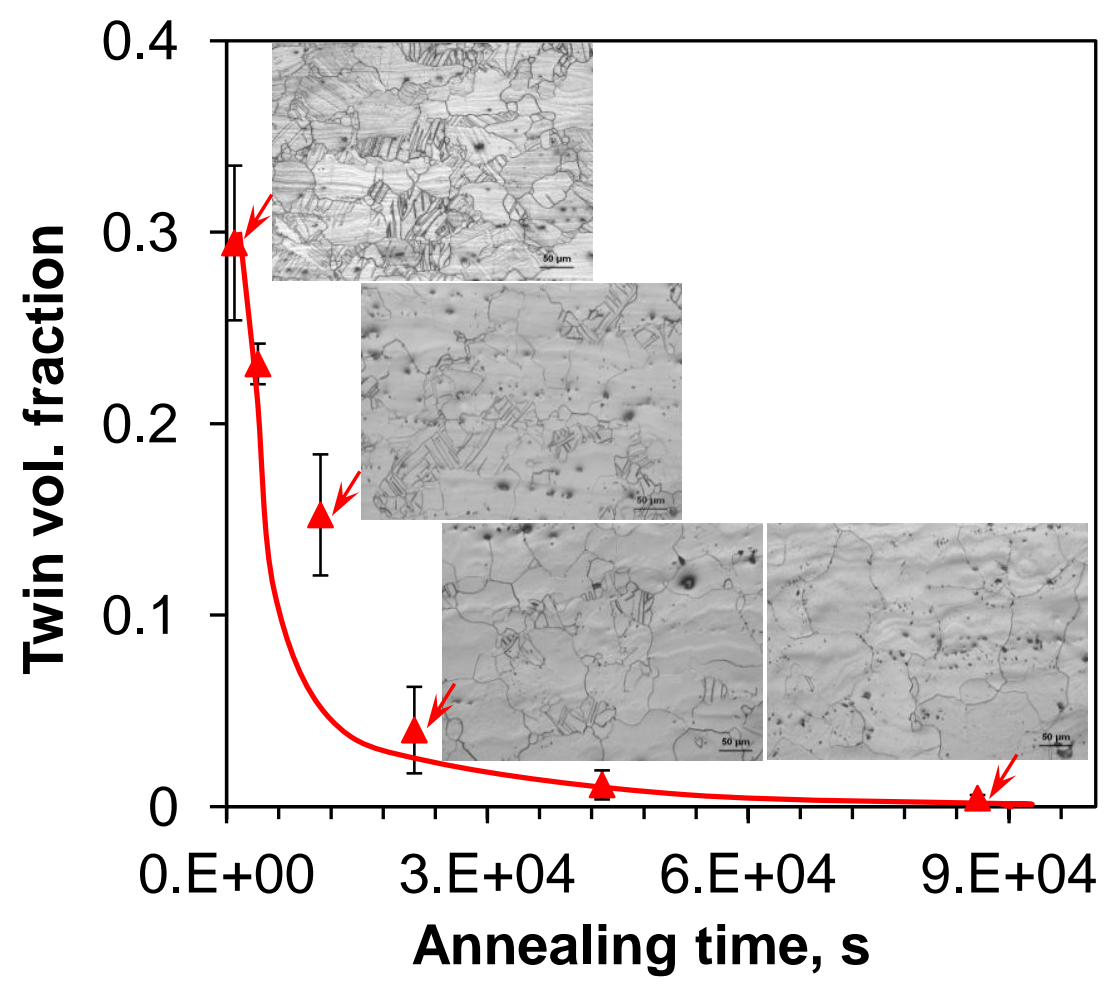

Figure 7.6: Volume fraction of extension twins vs. annealing time at a constant annealing temperature of $450^{\circ} \mathrm{C}$ for the $4.3 \%$ pre-strained samples.

The decrease in the volume fraction of twins was associated with the recovery and recrystallization to form twin-free grains (Fig. 7.6). Extensive twinning arising from the prestrain resulted in a large number of twin boundaries, giving rise to a fairly high internal energy in the deformed alloy. Thus, recovery and recrystallization would occur at such a high temperature of $450^{\circ} \mathrm{C}$. Since the growth of recrystallized grains involves atomic diffusion - a time-dependent process, a longer annealing time would lead to more complete recrystallization and growth of twin-free grains. Similar microstructural changes were also reported by Yang et al. [208] during annealing of an AZ31 magnesium alloy after cold MDF at various annealing times. 


\subsection{Recrystallization Kinetics}

The kinetics of static recrystallization of the $4.3 \%$ pre-compressed AM30 alloy were studied at an annealing temperature of $450^{\circ} \mathrm{C}$ in a time range of $0.25 \mathrm{~h}$ to $60 \mathrm{~h}$. The fraction of recrystallization $X_{r}$ was measured using the point counting method applied to different microstructures, such as those in Fig. 7.6, using the following equation:

$$
X_{r}=\frac{V_{f o}-V_{f t}}{V_{f t}},
$$

where $V_{f o}$ is the initial volume fraction of twins after pre-compression without annealing and $V_{f t}$ is the volume fraction of twins after annealing at a given time. The curve of fractional recrystallization versus annealing time $\left(X_{r}-t\right.$ curve) for the pre-compressed samples is presented in Fig. 7.7(a), which exhibited a sigmoidal shape as well. Similar sigmoidal curves were reported for cold deformed cubic materials [187]. The $X_{r}-t$ curve in Fig. 7.7(a) showed a rapid increase after an incubation period early in the annealing time, followed by a gradual increase, approaching $X r=1$ at longer annealing times. It is generally known that the kinetics of recrystallization could be described by the Johnson-Mehl-Avrami-Kolmogorov (JMAK) model where the relationship between recrystallization fraction $X_{r}$ and $t$ could be written as [187],

$$
X_{r}=1-\exp \left(-\eta t^{m}\right)
$$

where $\eta$ is a material constant, $t$ is the annealing time and $m$ is the JMAK exponent, which is equal to 4 if all the assumptions on which the model were built are satisfied [189]. 

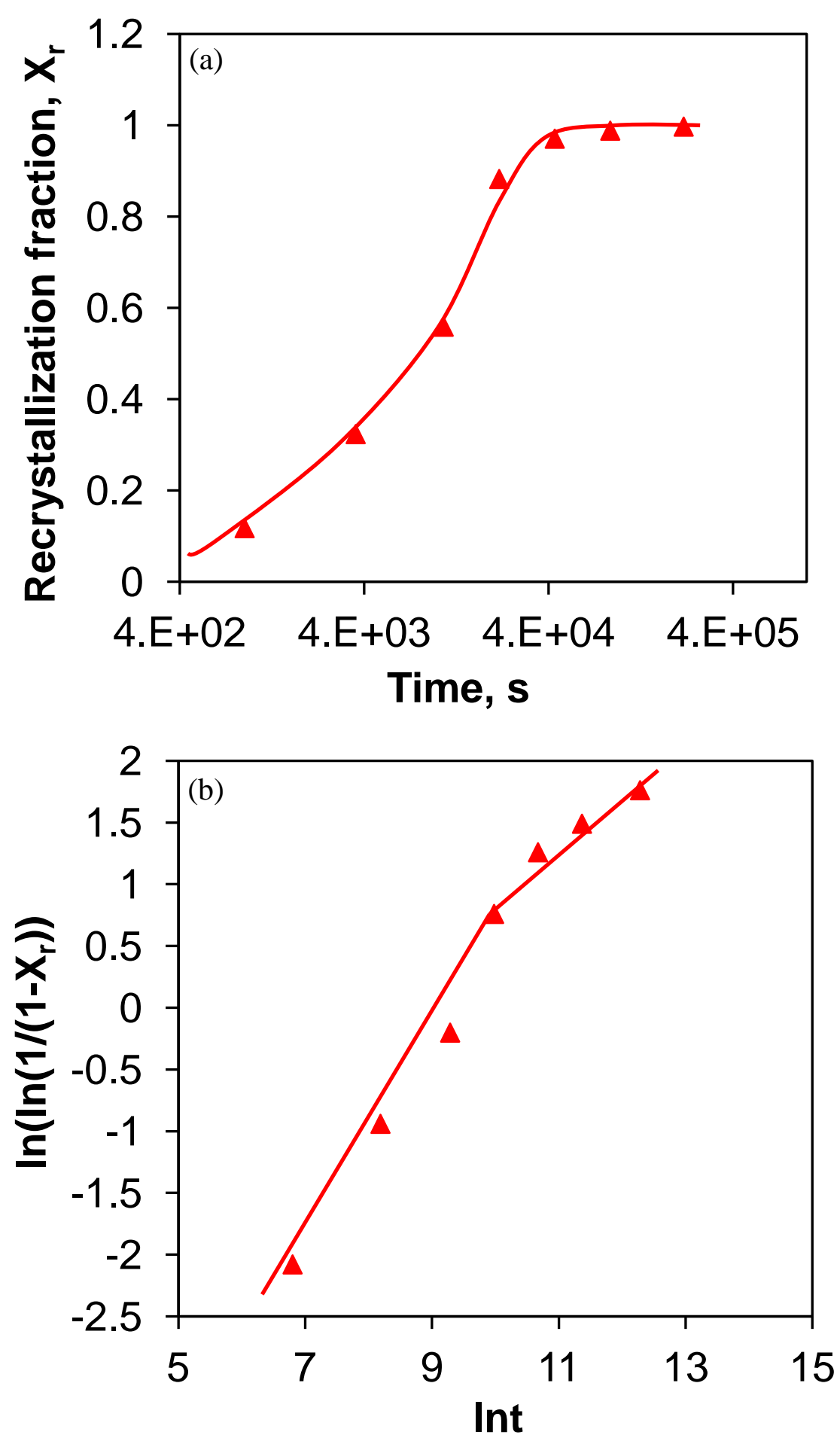

Figure 7.7: (a) Recrystallization fraction $\left(X_{r}\right)$ vs. time and (b) JMAK plot showing the recrystallization kinetics of the $4.3 \%$ pre-strained $\mathrm{AM} 30$ alloy during annealing at $450^{\circ} \mathrm{C}$ using a metallographic observation method. 
The JMAK exponent $(m)$ can be obtained from the linear relationship of the logarithm of $\ln \{1 /(1$ $\left.\left.X_{r}\right)\right\}$ and $\ln (t)$ as shown below,

$$
\ln \left(\ln \left(\frac{1}{1-X_{r}}\right)\right)=\ln \eta+m \ln t,
$$

Fig. 7.7(b) shows the JMAK exponent value $m$ as 0.87 or $\sim 1$ for the pre-compressed samples after annealing at $450^{\circ} \mathrm{C}$ at various times. The JMAK plot is approximated by a linear relationship in the early stages of annealing, but always breaks down at longer times, leading to lower values of the exponents, which was also reported by Yang et al. [207,208]. The value of the exponent close to 1 is very often observed in low carbon cold rolled steels [209,210]. This value is lower than that reported by $\mathrm{Su}$ et al. [189] which was 1.6, for a compressed AZ31 magnesium alloy at a strain of 0.1 and then annealed at $200^{\circ} \mathrm{C}$. The reason for the lower $m$ may be a result of non-random recrystallization sites present in the deformed material [187,189]. It also suggests that the recrystallization kinetics is more strongly influenced by the annealing temperature than time, in agreement with the results shown in Figs 7.3 and $7.5(\mathrm{~b}, \mathrm{c})$. According to Eq. (7.4), a small decrease in annealing time can also result in a considerable increase of the value of $m$ [204]. Humphreys and Hatherly [187] noted that the inhomogeneity of deformed microstructures that is caused by plastic deformation may play an important role on the break of the JMAK plots as well as the lower values of the exponent at longer annealing times. As the sample was plastically deformed by $4.3 \%$ pre-compression, the inhomogenity in the microstructure would caused a non-random distribution of nucleation sites and stored internal energy due to twin formation. As a result, recrystallization takes place more easily and the JMAK exponent reduces at longer annealing times. 


\subsection{Texture Development}

Fig. 7.8 shows the (0001) and (1010) pole figures, illustrating the texture of the $4.3 \%$ precompressed AM30 alloy after annealing at $450^{\circ} \mathrm{C}$ for (a) $3 \mathrm{~h}$, and (b) $12 \mathrm{~h}$, respectively. In comparison with the texture presented for a $4.3 \%$ pre-strained sample without annealing in Fig. 4.7(b), it was seen that after annealing at $450^{\circ} \mathrm{C}$ for $3 \mathrm{~h}$ (Fig. $7.8(\mathrm{a})$ ), a somewhat more random distribution of the basal (0001) poles was occurred, despite an equivalent maximum intensity within the experimental scatter. The texture became weaker with increasing annealing time to 12h, as shown in Fig. 7.8(b), where the basal (0001) poles showed a more random/scattered distribution towards both the ED and RD with an intensity of 4.6 MRD and the prismatic (1010) poles with an intensity of 2.4 MRD towards the RD. The texture results after annealing at different times corresponded well to the microstructure presented in Fig. 7.6.

As mentioned above and also discussed by a number of authors $[9,19,83,98,129,112]$, during compression along the ED $\{10 \overline{1} 2\}$ extension twinning led to a $86.3^{\circ}$ rotation of basal planes in the twin with respect to the basal planes in the parent grain. The texture presented in Fig. 4.7(b) showed such a significant change in orientation as compared to the un-deformed samples in Fig. 4.1(b). When the annealing time increased from $3 \mathrm{~h}$ to $12 \mathrm{~h}$, the microstructure examinations revealed a disappearance of twins (Fig. 7.6), which also supported the weak texture shown in Fig. 7.8(b). Similar microstructure development involving twin disappearance after long annealing times [207,208] and texture weakening [108,207,211] was reported as well, where the cold deformed magnesium alloy was also subjected to annealing at varying temperatures and 
times. The mechanism of this texture weakening together with the twin disappearance will be explained later.

(a)
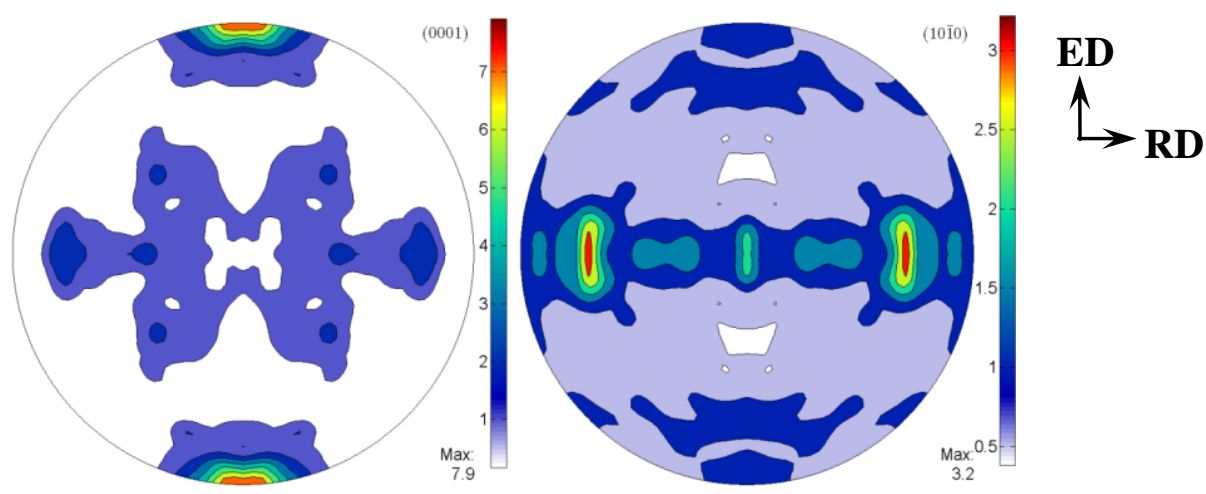

(b)
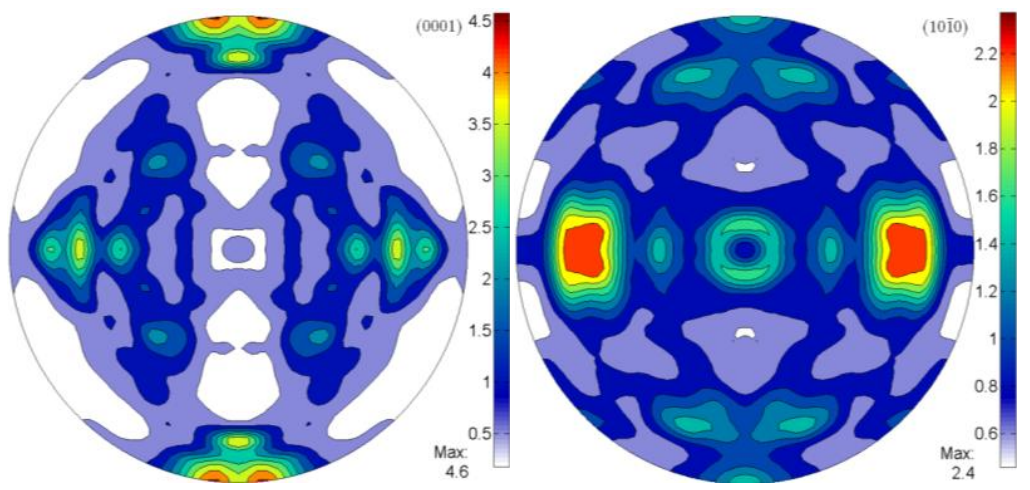

Figure 7.8: (0001) and (10ī0) pole figures of the $4.3 \%$ pre-compressed and subsequently annealed samples at $450^{\circ} \mathrm{C}$ for (a) $3 \mathrm{~h}$ and (b) $12 \mathrm{~h}$, respectively, where ED indicates the extrusion direction and RD stands for the radial direction.

The microstructure and the corresponding texture development after $4.3 \%$ re-compression of the 4.3\% pre-compressed and subsequently annealed samples are shown in Figs. 7.9 and 7.10 respectively. The microstructure in Fig. 7.9(a) showed the formation of some new twins after the second step compression of the annealed sample, as compared to Fig. 7.6 after annealing at $450^{\circ} \mathrm{C}$ for $3 \mathrm{~h}$. This was reflected by the slight increase in the intensity of (0001) poles along the ED after re-compression, i.e., 8.6 MRD in Fig. 7.10(a) vs. 7.9 MRD in Fig. 7.8(a). 

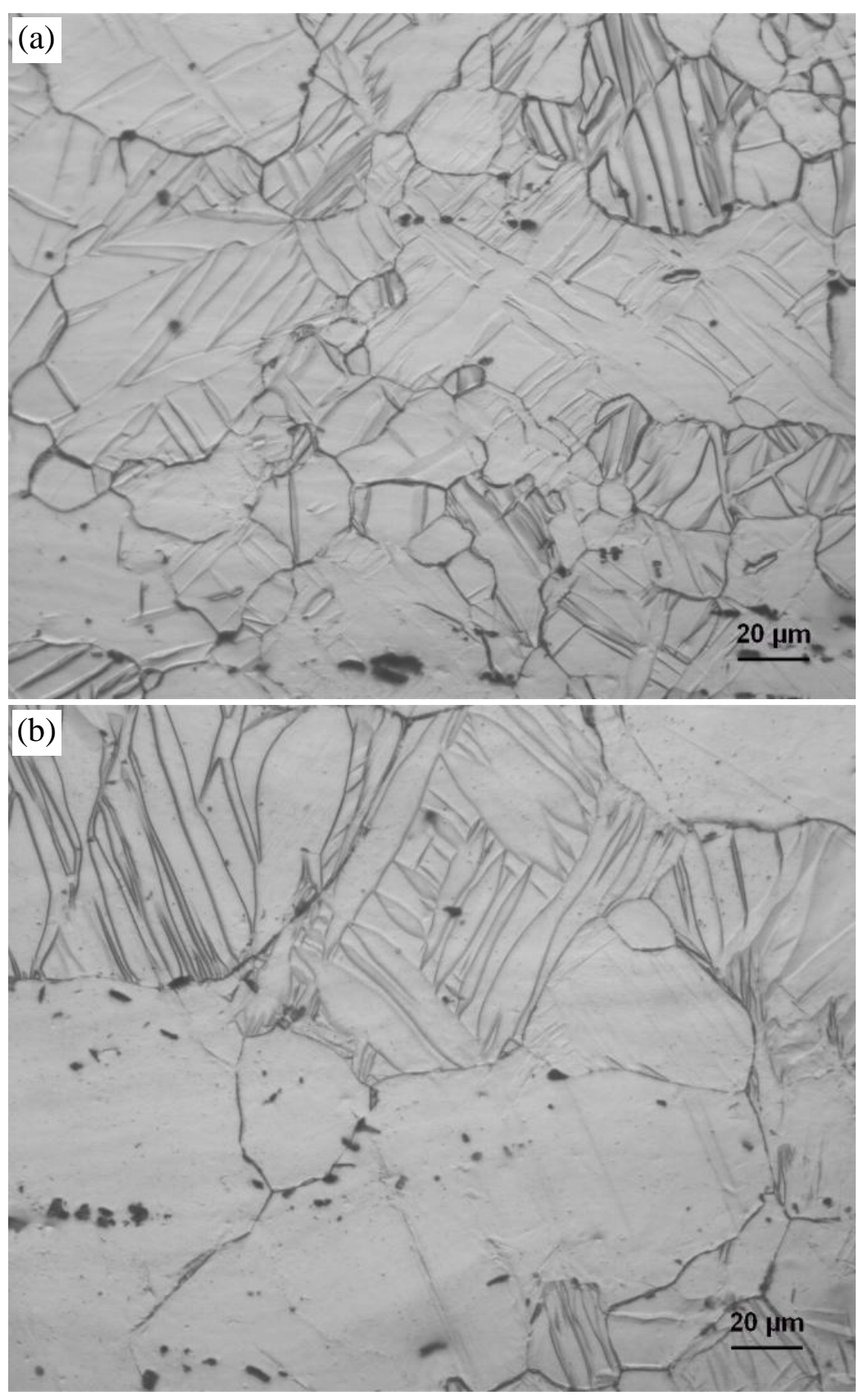

Figure 7.9: Microstructure of the $4.3 \%$ re-compressed sample after annealing of $4.3 \%$ prestrained samples at $450^{\circ} \mathrm{C}$ for (a) $3 \mathrm{~h}$ and (b) $12 \mathrm{~h}$, respectively.

While the same rotation of the $c$-axis of grains towards the anti-compression direction was observed during the second-step compressed sample (Fig. 7.10(a)), it had a less intense rotation 
of basal (0001) poles, compared with those after a continuous or interrupted two-step compression to an equivalent total strain level of $\sim 8 \%$ without any annealing, as presented in our previous study [169], where a basal (0001) pole intensity of 12 MRD and 9.6 MRD was obtained, respectively.

(a)

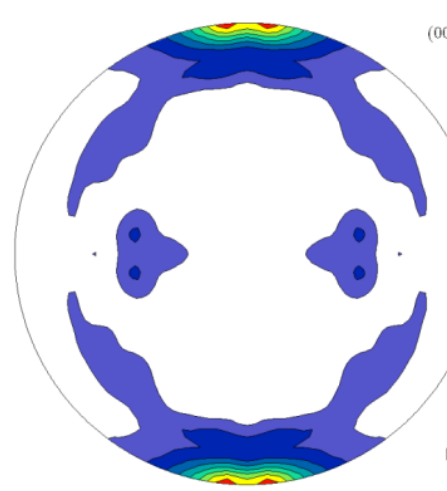

(b)
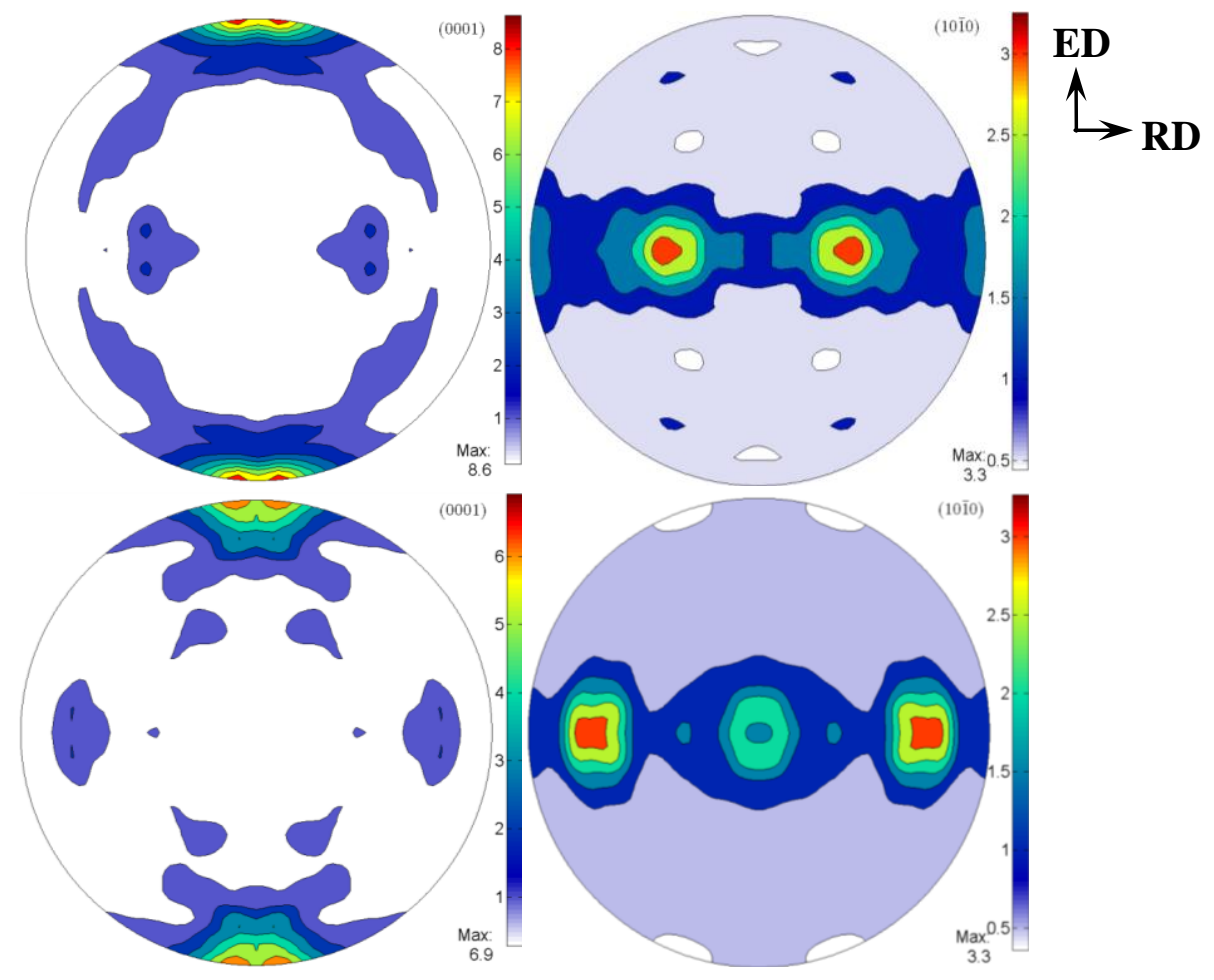

Figure 7.10: (0001) and (1010) pole figures of the $4.3 \%$ re-compressed sample after annealing of $4.3 \%$ pre-strained samples at $450^{\circ} \mathrm{C}$ for (a) $3 \mathrm{~h}$ and (b) $12 \mathrm{~h}$, respectively.

At longer annealing time of $12 \mathrm{~h}$, while some new twins formed as well from the recrystallized grains during re-compression (Fig. 7.9(b) vs. Fig. 7.6) the basal (0001) pole intensity became higher (6.9 MRD in Fig. 7.10(b) vs. 4.6 MRD in Fig. 7.8(b)), fewer twins were seen (Fig. 7.9(b) vs. Fig. 7.9(a)) and the basal (0001) pole intensity was lower (6.9 MRD in Fig. 710(b) vs. 8.6 
MRD in Fig. 7.10(a)). This suggests that annealing temperature and time had a significant effect on microstructure and texture formation.

The annealing temperature and time are important as they govern recovery, recrystallization, and grain growth. When the annealing time was $3 \mathrm{~h}$, a temperature of $450^{\circ} \mathrm{C}$ might be insufficient to modify microstructure significantly, and some twins still remained in grains (Fig. 7.6). Similar results were observed by Lu et al. [141] who explained that energy produced by low annealing temperatures was insufficient to modify cold deformed magnesium. Yang et al. [208] also reported that the number of high density twins gradually decreased and concurrently their widths broadened with increasing annealing time, suggesting the frequent occurrence of recovery in grain interiors during annealing. On the other hand, Li et al. [108] explained that $\{10 \overline{2}\}$ extension twins possessed mobile twin boundaries, and thus they could become broader under higher annealing temperatures. Therefore, the deformation cannot lead to a high strain accumulation at $\{1012\}$ extension twin boundaries. In this sense twin boundaries may not serve as active nucleation sites for the static recrystallization. However, in the present AM30 Mg alloy pre-compressed to $4.3 \%$, almost all grains have twins that frequently intersect with one another, which can result in high strain concentrations around their intersections and grain boundaries and later have a high potentiality for the nucleation sites of static recrystallization [207,208].

A schematic diagram is shown in Fig. 7.11 to describe the static recrystallization process during annealing of the pre-compressed AM30 alloy. Fig. 7.11(a) shows preferential nucleation sites at grain boundaries and within twins due to the higher energy. As the annealing process going on, small new and twin-free grains form in the twins and at grain boundaries which are presented in 
Fig. 7.11(b). New grains grow mainly within twins and typically do not grow laterally out of twin boundaries [108]. While contraction twin boundaries are generally immobile, basal slip, extension twinning or other types of interactions may destroy the immobile boundaries and allow the new grains to form and grow towards the deformed matrix and will result in full recrystallization at a sufficiently high temperature or sufficiently long annealing time, as shown in Fig. 7.11(c). It could be considered that the early stage of annealing for the cold-deformed $\mathrm{Mg}$ alloy may act as an incubation period for new grain formation at the intersections of twins and grain boundaries accompanied by dislocation rearrangement and annihilation.

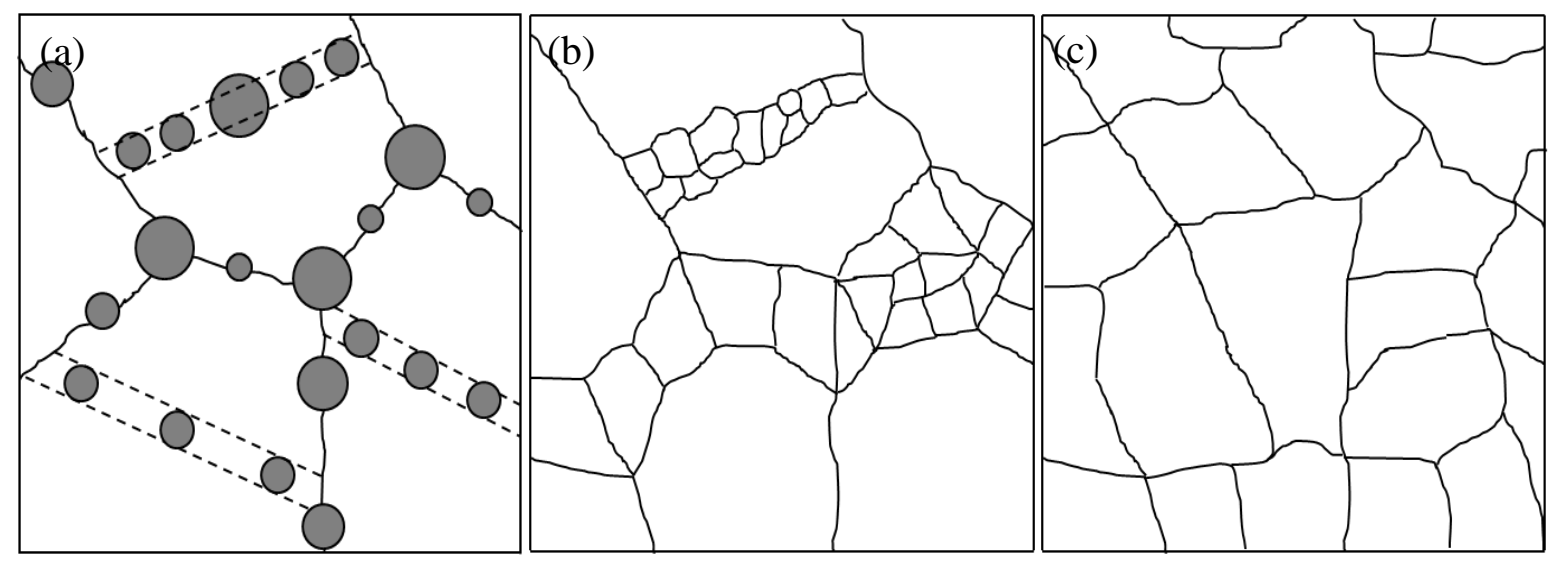

Figure 7.11: Schematic illustration of recrystallization process during annealing of a compressed AM30 alloy: (a) the beginning of recrystallization with preferential nucleation in twins and grains, (b) formation of new small grains within twins and at grain boundaries, (c) recrystallized grains.

Further annealing can result in the formation of new grains, followed by their large-distance migration or grain growth until their impingement. During recrystallization, the texture became increasingly weaker with a more random/scattered distribution, as seen from Fig. 7.8 and also 
reported in $[108,207,211,212]$. At a pre-strain of $4.3 \%$, the twinned grains are not fully encompassed by twins, and therefore there must also be recrystallized grains formed from the untwinned parent regions. The recrystallized grains within twins and untwinned regions have different orientations that eventually break the strong texture and make it weaken. During recompression to $4.3 \%$ as well, twin formation was not as intense as shown in Fig. 7.9(b) which resulted in a weaker texture in Fig. 7.10(b). This might be due to unfavorable $c$-axis orientation for twinning after recrystallization and eventually decreased the extent of strain hardening stage B (Fig. 7.4) and increased the fracture strain (Fig. 7.2(a)).

\subsection{Summary}

In this chapter, the recrystallization kinetics and the re-compression properties of the precompressed and annealed AM30 Mg alloy were studied with a focus on the strain hardening behavior, microstructure and texture development.

After a $4.3 \%$ pre-compression along the extrusion direction (ED), most grains twinned and the $c$ axes of the twinned regions were rotated towards the ED (i.e., always towards the compression direction). A distinct stage B of strain hardening was present, which was characterized by an increasing strain hardening rate.

The subsequent annealing of the pre-compressed samples at varying temperatures and times led to recovery, recrystallization and grain growth. Recrystallization kinetics could be well described using the Johnson-Mehl-Avrami-Kolmogorov (JMAK) model. 
As the annealing time increased at a temperature of $450^{\circ} \mathrm{C}$, the ultimate compressive strength (UCS) and the slope of strain hardening stage B decreased, while the compressive yield stress (CYS) remained nearly the same. With increasing annealing temperature, CYS, UCS, and the extent of stage B as well as the slope of stage B all decreased, whereas the fracture strain increased. However, the UCS remained basically unchanged when the pre-compressed samples were annealed at $250^{\circ} \mathrm{C}$ for $3 \mathrm{~h}$.

With increasing annealing time, the twins in the pre-compressed samples were observed to disappear gradually. This was reflected by a decreased volume fraction of twins and weakened texture which became more randomly distributed as well. The recrystallized grains within twins and untwinned regions starting from the grain boundaries had different orientations which caused the strong texture to split and weaken. 


\section{CHAPTER 8}

\section{Conclusions and Future Work}

\subsection{Conclusions}

Based on the studies of the mechanical behavior and the associated plastic deformation mechanisms under monotonic compressive loading of the extruded AM30 magnesium alloy, the following conclusions can be drawn:

(1) The plastic deformation of extruded AM30 magnesium alloy by compression in the extrusion direction can be characterized by the occurrence of extension twinning. There is a sigmoidal relationship between the twin growth and the strain. The twin boundaries merged or disappeared with increasing compressive strain.

(2) Twin formation can be influenced by the inclination of basal texture and active twin variants can be identified using the Schmid factor. The active twin variants reflected by the high Schmid factor value were significantly dependent on the activation mode (i.e., compression perpendicular to the $c$-axis) which was governed by the combination of the crystallographic lattice orientation with the applied loading direction.

(3) Pre-strain has a significant effect on twinning, twin growth and de-twinning. The two-step compression in the ED-ED sample showed a lower extent of twin growth than that in the one-step continuous compression at an equivalent total strain level. In the ED-TD 
compressed samples, re-compression along the TD after pre-strain along the ED led to the co-existence of two seemingly opposite phenomena, i.e., the formation of new secondary twins and de-twinning. In the subsequent second-step compression along the ND, backtwinning (de-twinning) occurred.

(4) Static recrystallization occurred during annealing of the pre-compressed samples at different temperatures and times. With increasing annealing time, the twins in the precompressed samples were observed to disappear gradually. The recrystallized grains within twins and untwinned regions showed less intense twin formation during recompression.

(5) A sigmoidal true stress-true strain curve together with three distinct stages of strain hardening rate was observed during compression along the extrusion direction. The increase of in-plane loading direction with respect to the ED showed nearly constant YS and UCS with increased fracture strain and a decreasing trend in the slope of the strain hardening rate curve in stage $\mathrm{B}$.

(6) The pre-strain amount and the reloading direction influenced the compressive properties in all cases of ED-ED, ED-TD and ED-ND compressed samples. With increasing pre-strain the YS linearly increased in ED-ED and ED-TD samples, whereas it decreased in ED-ND sample. An increasing trend of UCS is observed in ED-TD and ED-ND samples unlike ED-ED sample. With increasing pre-strain the sigmoidal shape of the flow curves and three distinct stages of strain hardening rate are obvious in ED-TD and ED-ND samples. 
(7) During re-compression along the ED, pre-compressed and annealed samples at different temperatures exhibited decreasing trends of YS, UCS as well as the slope of the strain hardening rate curve, whereas fracture strain increased.

(8) The compressive deformation along the ED showed a remarkable change in texture components, where the initial basal texture components $\{0001\}<2 \overline{1} \overline{1} 0>$ and $\{0001\}$ $<10 \overline{1} 0>$ were observed to fade away, while $\{\overline{1} 2 \overline{1} 0\}<0001>$ and $\{01 \overline{1} 0\}<0001>$ texture components intensified with increasing compressive strain.

(9) Texture weakening was achieved through the pre-compression in the ED and subsequent re-compression along the TD and ND. The formation of new twins in the second-step compression along the TD and ND caused the $c$-axes of hcp unit cells in some grains reverting back to the as-extruded state. A weakened texture was also observed from the recrystallization of the pre-compressed sample.

\subsection{Original Contributions}

Although the occurrence of deformation twinning in wrought magnesium alloys has been well recognized, its effect on the mechanical behavior under monotonic loading has not yet been well understood. This research conducted fundamental studies on the underlying deformation mechanisms (with a special focus on twinning) and mechanical behavior in wrought magnesium alloys, which are essential for the implementation of lightweight structural applications in the manufacturing sectors, since twinning plays a significant role in the deformation and failure of 
magnesium alloys. The study of the close relationship between the crystallographic textures and plastic deformation mechanisms in wrought magnesium alloys, which reciprocally influence each other, provides valuable information for the general understanding of the plastic deformation of polycrystalline materials with hexagonal structures, and will be beneficial for designing optimal alloying and forming schemes to manufacture reliable lightweight magnesium alloys for use in the automotive and aerospace industries. The main findings from this dissertation can be summarized as follows:

Twin formation can be influenced by the inclination of basal texture and active twin variants can be identified using the Schmid factor. A sigmoidal true stress-true strain curve together with three distinct stages of strain hardening rate was observed during compression along the ED. The increase of in-plane loading direction with respect to the ED showed nearly constant YS and UCS with increased fracture strain and a decreasing trend in the slope of the strain hardening rate curve. These findings have led to the following publications:

Scripta Materialia, vol. 67(2), pp. 165-168, 2012. [135]

Materials Science and Engineering A, vol. 582, pp. 63-67, 2013. [129]

Materials Science Forum, vol. 783-786, pp. 363-368, 2014. [176]

Journal of Materials Science and Technology, (Accepted). [171]

Pre-strain has a significant effect on twinning, twin growth and de-twinning. In the ED-TD compressed samples, re-compression along the TD after pre-strain along the ED led to the co-existence of new secondary twins and de-twinning. An increasing trend of UCS is 
observed in ED-TD and ED-ND samples unlike ED-ED sample. Texture weakening was achieved through the pre-compression in the ED and subsequent re-compression along the TD and ND. These findings have led to the following publications:

Materials Science and Engineering A, vol. 596, pp. 134-144, 2014 [169]

Materials Science and Engineering A, vol. 605, pp. 73-79, 2014. [125]

The recrystallized grains within twins and un-twinned regions showed less intense twin formation during recompression. A decreasing trend of YS, UCS as well as the slope of the strain hardening rate curve was observed, whereas fracture strain increased. A weakened texture was also observed from the recrystallization of the pre-compressed sample. These findings have led to the following publications:

Journal of Alloys and Compounds, vol. 611, pp. 341-350, 2014. [212]

\subsection{Recommendations for Future Work}

Magnesium alloys are ideal model materials for studying twinning, because of near elastic isotropy and near isotropic coefficients of thermal expansion, which results in negligible residual stress after hot deformation. Furthermore, wrought magnesium alloys usually have a very strong basal texture. Therefore, by tailoring the initial texture and applied loading relative to the texture, it is possible to introduce a stress state where extension twinning is the major deformation mechanism. The following future investigations could contribute to an in-depth mechanistic understanding of the unusual plastic deformation behavior in wrought magnesium alloys during monotonic and/or cyclic loading: 
(1) The strain distribution and hardening within polycrystalline samples of AM30 needs to be measured on a more localized scale (i.e., at the grain level). A detailed investigation combining EBSD to map the crystal orientations prior to deformation, digital image correlation (DIC) to track the local strain distribution during mechanical testing, and nanoindentation to measure mechanical properties (specifically indentation yield stress) on particular orientations at intermediate strains would prove to be highly valuable in clarifying the roles at various orientations during deformation.

(2) Polycrystal plasticity modeling combined with TEM observations will be helpful to understand the exceptional hardening behavior in magnesium alloys.

(3) Grain-grain interaction using 3D XRD and/or tomography could provide the necessary clues to better understand the de-twinning mechanisms in magnesium alloys.

(4) Grain-level twinning and de-twinning behavior in wrought magnesium alloys using in-situ synchrotron microdiffraction could be used to study the grain reorientation and the development of internal-strain within individual grains, such that the orientation and full strain tensor can be determined within a single grain as a function of strain, which are untwinned, partially twinned and completely twinned.

(5) A detailed investigation on the contribution of both extension and contraction twins on static recrystallization and the role of these twins on recrystallization texture needs to be done. The annealing should be interrupted at various times to allow for EBSD to identify 
the orientations present (i.e., determine what, if any, areas from the previous structure have recrystallized). Further deformation of the pre-deformed and annealed sample should be done using different loading direction to observe the effect of recrystallization on the texture evolution. 


\section{APPENDIX A}

\section{Calculation of Schmid Factor}

\section{A.1 Calculation Method:}

To calculate the Schmid Factor, some sectional views of the geometrical projection of Fig. 5.2 are shown in Fig. A1. Where, FR' is the twin normal on FN (Fig. A1(a)), which intercepts the extended NR at point $\mathrm{R}^{\prime}$, a projection from $\mathrm{R}^{\prime}$ to the sample surface intercepts at point $\mathrm{R}^{\prime \prime}$ (Fig. 5.2).

(a)

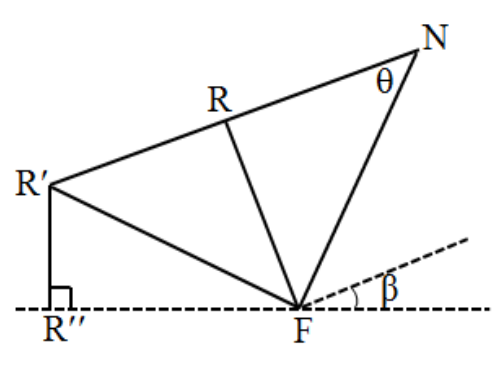

(b)

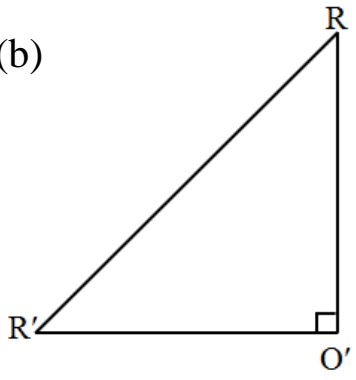

(c)

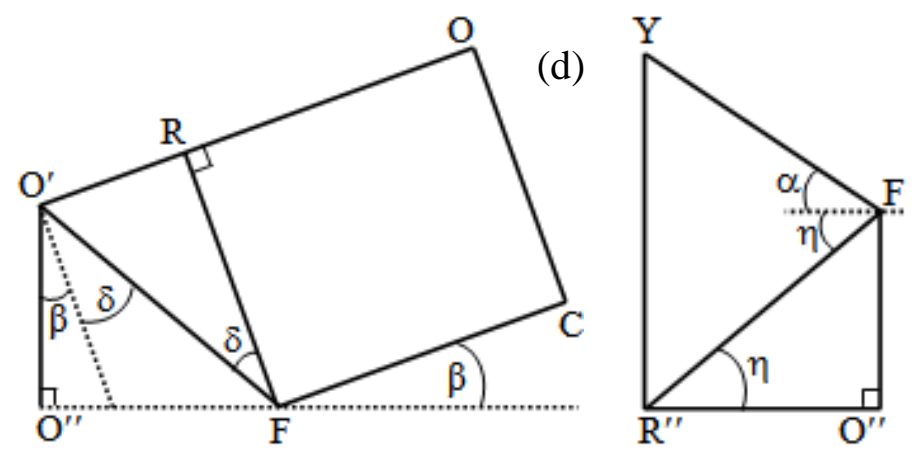

Figure A1: (a-d) Some sectional views of Fig. 5.2 used to calculate Schmid Factor. 
A perpendicular line in the extrusion direction (ED), passes through the point $\mathrm{R}^{\prime}$, intercepts the extended $\mathrm{OR}$ at point $\mathrm{O}^{\prime}$ and makes a right triangle $\Delta \mathrm{RR}^{\prime} \mathrm{O}^{\prime}$ (Fig. $\mathrm{A} 1(\mathrm{~b})$ ). A projection from $\mathrm{O}^{\prime}$ to the sample surface intercepts at point $\mathrm{O}^{\prime \prime}$ (Fig. A1(c)) and $\mathrm{R}^{\prime} \mathrm{R}^{\prime \prime}=\mathrm{O}^{\prime} \mathrm{O}^{\prime \prime}$. Fig. A1(d) shows the calculation of $\eta$.

$$
\begin{gathered}
\Delta F Z Y: F Y=\frac{a \sqrt{3}}{2 \cos \alpha}, \\
\Delta F B N: F N=\sqrt{3 a^{2}+c^{2}} \\
\Delta F R S: F S=\sqrt{c^{2}+(9 / 4) a^{2}} \\
\Delta F S S^{\prime}: \gamma=\sin ^{-1}\left(\frac{c}{F S}\right)=\sin ^{-1}\left(\frac{c}{\sqrt{c^{2}+(9 / 4) a^{2}}}\right), \\
\Delta F S F^{\prime}: F^{\prime} S=F S \cdot \sin (\beta+\gamma)=\sqrt{c^{2}+(9 / 4) a^{2}} \cdot \sin (\beta+\gamma), \\
F F^{\prime}=F S \cdot \cos (\beta+\gamma)=\sqrt{c^{2}+(9 / 4) a^{2} \cdot \cos (\beta+\gamma),} \\
N N^{\prime}=F^{\prime} S=\sqrt{c^{2}+(9 / 4) a^{2}} \cdot \sin (\beta+\gamma), \\
X N^{\prime}=F F^{\prime}=\sqrt{c^{2}+(9 / 4) a^{2} \cdot \cos (\beta+\gamma),} \\
X Y^{\prime}=Z Y=F Y \cdot \sin \alpha=\frac{a \sqrt{3}}{2} \cdot \tan \alpha, \\
Y Y^{\prime}=a \sqrt{3}, \\
Y^{\prime} N^{\prime}=X N^{\prime}-X Y^{\prime}=\sqrt{c^{2}+(9 / 4) a^{2} \cdot \cos (\beta+\gamma)-\frac{a \sqrt{3}}{2}} \cdot \tan \alpha,
\end{gathered}
$$




$$
\begin{aligned}
& \Delta Y Y^{\prime} N^{\prime}: N^{\prime} Y=\sqrt{Y Y^{\prime 2}+Y^{\prime} N^{\prime 2}} \\
& =\sqrt{3 a^{2}+\left(c^{2}+(9 / 4) a^{2}\right) \cdot \cos ^{2}(\beta+\gamma)+\frac{3 a^{2}}{4} \cdot \tan ^{2} \alpha-a \sqrt{3} \cdot \sqrt{c^{2}+(9 / 4) a^{2}} \cdot \cos (\beta+\delta) \cdot \tan \alpha}
\end{aligned}
$$

$$
\begin{aligned}
& \Delta N N^{\prime} Y: Y N=\sqrt{N N^{\prime 2}+N^{\prime} Y^{2}} \\
& =\sqrt{c^{2}+(9 / 4) a^{2}+3 a^{2}\left(1+\frac{\tan ^{2} \alpha}{4}\right)-a \sqrt{3} \cdot \sqrt{c^{2}+(9 / 4) a^{2}} \cdot \cos (\beta+\delta) \cdot \tan \alpha}, \\
& \Delta F Y N: \cos \varphi=\frac{(F Y)^{2}+(F N)^{2}-(Y N)^{2}}{2(F Y)(F N)} \\
& =\frac{2 \cdot \sqrt{c^{2}+(9 / 4) a^{2}} \cdot \cos \left(\beta+\sin ^{-1}\left(\frac{c}{\sqrt{c^{2}+(9 / 4) a^{2}}}\right)\right) \cdot \sin \alpha-a \sqrt{3} \cdot \cos \alpha}{2 \cdot \sqrt{3 a^{2}+c^{2}}} \\
& \Delta F R N: \theta=\tan ^{-1}\left(\frac{c}{a \sqrt{3}}\right), \\
& \triangle F R R: F R^{\prime}=\frac{c}{\cos \theta}, \\
& R R^{\prime}=c \cdot \tan \theta, \\
& \Delta R R^{\prime} O^{\prime}: R O^{\prime}=\frac{c \sqrt{3} \tan \theta}{2}, \\
& R^{\prime} O^{\prime}=R^{\prime \prime} O^{\prime \prime}=\frac{c \cdot \tan \theta}{2}, \\
& \Delta F R O^{\prime}: F O^{\prime}=\sqrt{F R^{2}+R O^{\prime 2}}=c \sqrt{1+\frac{3 \tan ^{2} \theta}{4}}
\end{aligned}
$$




$$
\begin{aligned}
& \delta=\tan ^{-1}\left(\frac{c}{2 a}\right) \\
& \Delta F O^{\prime} O^{\prime \prime}: O^{\prime} O^{\prime \prime}=R^{\prime} R^{\prime \prime}=F O^{\prime} \cdot \cos (\beta+\delta)=c \sqrt{1+\frac{3 \tan ^{2} \theta}{4}} \cdot \cos (\beta+\delta) \\
& F O^{\prime \prime}=F O^{\prime} \cdot \sin (\beta+\delta)=c \sqrt{1+\frac{3 \tan ^{2} \theta}{4}} \cdot \sin (\beta+\delta) \\
& \Delta F R^{\prime \prime} O^{\prime \prime}: F R^{\prime \prime}=\sqrt{F O^{\prime \prime}+R^{\prime \prime} O^{\prime \prime}}=\frac{c}{2} \sqrt{\left(4+3 \tan ^{2} \theta\right) \cdot \sin ^{2}(\beta+\delta)+\tan ^{2} \theta} \\
& \eta=\tan ^{-1}\left(\frac{F O^{\prime \prime}}{R^{\prime \prime} O^{\prime \prime}}\right)=\tan ^{-1}\left(\frac{\sqrt{4+3 \tan ^{2} \theta} \cdot \sin (\beta+\delta)}{\tan \theta}\right) \\
& R^{\prime \prime} Y=\sqrt{\left(F Y^{2}+F R^{\prime \prime 2}-2 \cdot F Y \cdot F R^{\prime \prime} \cdot \cos (\alpha+\eta)\right.} \\
& =\left(\begin{array}{l}
\frac{3 a^{2}}{4 \cos ^{2} \alpha}+\frac{c^{2}}{4}\left(\left(4+3 \tan ^{2} \theta\right) \cdot \sin ^{2}(\beta+\delta)+\tan ^{2} \theta\right) \\
-\frac{a \cdot c \sqrt{3}}{2 \cos \alpha} \sqrt{\left(4+3 \tan ^{2} \theta\right) \cdot \sin ^{2}(\beta+\delta)+\tan ^{2} \theta} \cdot \cos (\alpha+\eta)
\end{array}\right)^{1 / 2} \\
& R^{\prime} Y=\sqrt{R^{\prime} R^{\prime \prime}+R^{\prime \prime} Y^{2}} \\
& =\left(\frac{c^{2}}{\cos ^{2} \theta}+\frac{3 a^{2}}{4 \cos ^{2} \alpha}-\frac{a . c \sqrt{3}}{2 \cos \alpha} \sqrt{\left(4+3 \tan ^{2} \theta\right) \cdot \sin ^{2}(\beta+\delta)+\tan ^{2} \theta} \cdot \cos (\alpha+\eta)\right)^{1 / 2}(\mathrm{~A} .27) \\
& \cos \lambda=\frac{(F Y)^{2}+(F R)^{2}-\left(R^{\prime} Y\right)^{2}}{2(F Y)\left(F R^{\prime}\right)} \\
& =\frac{\sqrt{\left(4+3 \tan ^{2} \theta\right) \cdot \sin ^{2}(\beta+\delta)+\tan ^{2} \theta} \cdot \cos (\alpha+\eta) \cdot \cos \theta}{2}
\end{aligned}
$$




\section{REFERENCES}

1. J. Murray and D. King, “Oil's tipping point has passed,” Nature, vol. 481, pp. 433-435, 2012.

2. T.M. Pollock, "Weight loss with magnesium alloys," Science, vol. 328, pp. 986-987, 2010.

3. J.F. Nie, Y.M. Zhu, J.Z. Liu and X.Y. Fang, "Periodic segregation of solute atoms in fully coherent twin boundaries," Science, vol. 340, pp. 957-960, 2013.

4. M. McNutt, “Climate change impacts,” Science, vol. 341, p. 435, 2013.

5. C. Ash, E. Culotta, J. Fahrenkamp-Uppenbrink, D. Malakoff, J. Smith, A. Sugden and S. Vignieri, “Once and future climate change,” Science, vol. 341, pp. 472-473, 2013.

6. R.L. Revesz, P.H. Howard, K. Arrow, L.H. Goulder, R.E. Kopp, M.A. Livermore, M. Oppenheimer and T. Sterner, "Global warming: Improve economic models of climate change," Nature, vol. 508, pp. 173-175, 2014. .

7. Q. Schiermeier, "Water risk as world warms," Nature, vol. 505, pp. 10-11, 2014.

8. W.J. Joost, "Reducing vehicle weight and improving U.S. energy efficiency using integrated computational materials engineering,” JOM, vol. 64, pp. 1032-1038, 2012.

9. F.A. Mirza, D.L. Chen, D.J. Li and X.Q. Zeng, "Effect of strain ratio on cyclic deformation behavior of a rare-earth containing extruded magnesium alloy," Materials Science and Engineering, vol. 588, pp. 250-259, 2013.

10. N. Tahreen, D.L. Chen, M. Nouri and D.Y. Li, "Effects of aluminum content and strain rate on strain hardening behavior of cast magnesium alloys during compression," Materials Science and Engineering A, vol. 594, pp. 235-245, 2014. 
11. S.H. Park, S.G. Hong, J.H. Lee and C.S. Lee, "Multiple twinning modes in rolled Mg-3Al1Zn alloy and their selection mechanism," Materials Science and Engineering A, vol. 532, pp. 401-406, 2012.

12. X.Z. Lin and D.L. Chen, "Strain hardening and strain-rate sensitivity of an extruded magnesium alloy," Journal of Materials Engineering and Performance, vol. 17, pp. 894-901, 2008.

13. S. Begum, D.L. Chen, S. Xu and A.A. Luo, "Low cycle fatigue properties of an extruded AZ31 magnesium alloy,” International Journal of Fatigue, vol. 31, pp. 726-735, 2009.

14. S. Begum, D.L. Chen, S. Xu and A.A. Luo, "Effect of strain ratio and strain rate on low cycle fatigue behavior of AZ31 wrought magnesium alloy," Materials Science and Engineering A, vol. 517, pp. 334-343, 2009.

15. S. Begum, D.L. Chen, S. Xu and A.A. Luo, "Strain-controlled low cycle fatigue properties of a newly developed extruded magnesium alloy," Metallurgical and Materials Transactions A, vol. 39a, pp. 3014-3026, 2008.

16. Y. Kun, R. Shou-tai, W. Xiao-yan, W. Ri-chu, and L. Wen-ian, "Texture evolution of extruded AZ31 magnesium alloy sheets," Trans. Nonferrous Met. Soc. China, vol. 19, pp. 511-516, 2009.

17. F.A. Mirza, D.L. Chen, D.J. Li and X.Q. Zeng, "Cyclic deformation of rare-earth containing magnesium alloys," Advanced Materials Research, vol. 891-892, pp. 391-396, 2014.

18. F.A. Mirza, D.L. Chen, D.J. Li and X.Q. Zeng, "Effect of rare earth elements on deformation behavior of an extruded Mg-10Gd-3Y-0.5Zr alloy during compression," Materials and Design, vol. 46, pp. 411-418, 2013. 
19. F.A. Mirza, D.L. Chen, D.J. Li and X.Q. Zeng, "Low cycle fatigue of a rare-earth containing extruded magnesium alloy," Materials Science and Engineering A, vol. 575, pp. 65-73, 2013.

20. Y. Xin, M. Wang, Z. Zeng, M. Nie and Q. Liu, "Strengthening and toughening of magnesium alloy by $\{1012\}$ extension twins," Scripta Materialia, vol. 66, pp. 25-28, 2012.

21. S.H. Park, S.G. Hong and C.S. Lee, "In-plane anisotropic deformation behavior of rolled Mg-3Al-1Zn alloy by initial $\{10 \overline{1} 2\}$ twins," Materials Science and Engineering A, vol. 570, pp. 149-163, 2013.

22. S.-B. Yi, C.H.J. Davies, H.-G. Brokmeier, R.E. Bolmaro, K.U. Kainer and J. Homeyer, "Deformation and texture evolution in AZ31 magnesium alloy during uniaxial loading," Acta Materialia, vol. 54, pp. 549-562, 2006.

23. S.B. Yi, S. Zaefferer and H.-G. Brokmeier, "Mechanical behaviour and microstructural evolution of magnesium alloy AZ31 in tension at different temperatures," Materials Science and Engineering A, vol. 424, pp. 275-281, 2006.

24. R. Gehrmann, M.M. Frommert and G. Gottstein, "Texture effects on plastic deformation of magnesium," Materials Science and Engineering A, vol. 395, pp. 338-349, 2005.

25. Y. Xin, M. Wang, Z. Zeng, G. Huang and Q. Liu, "Tailoring the texture of magnesium alloy by twinning deformation to improve the rolling capability," Scripta Materialia, vol. 64, pp. 986-989, 2011.

26. Z. Keshavarz and M.R. Barnett, "EBSD analysis of deformation modes in Mg-3Al-1Zn," Scripta Materialia, vol. 55, pp. 915-918, 2006.

27. P. Cizek and M.R. Barnett, "Characteristics of the contraction twins formed close to the fracture surface in $\mathrm{Mg}-3 \mathrm{Al}-1 \mathrm{Zn}$ alloy deformed in tension," Scripta Materialia, vol. 59, pp. 959-962, 2008. 
28. B.H. Lee, S.H. Park, S.G. Hong, K.T. Park and C.S. Lee, "Role of initial texture on the plastic anisotropy of Mg-3Al-1Zn alloy at various temperatures," Materials Science and Engineering A, vol. 528, pp. 1162-1172, 2011.

29. P. Yang, Y. Yu, L. Chen and W. Mao, "Experimental determination and theoretical prediction of twin orientations in magnesium alloy AZ31," Scripta Materialia, vol. 50, pp. 1163-1168, 2004.

30. M.T. Pérez-Prado, J.A. del Valle and O.A. Ruano, "Grain refinement of Mg-Al-Zn alloys via accumulative roll bonding," Scripta Materialia, vol. 51, pp. 1093-1097, 2004.

31. A. Jiang, A. Godfrey, W. Liu and Q. Liu, "Microtexture evolution via deformation twinning and slip during compression of magnesium alloy AZ31," Materials Science and Engineering A, vol. 483-484, pp. 576-579, 2008.

32. Y.T. Zhu, X.L. Wu, X.Z. Liao, J. Narayan, L.J. Kecskes and S.N. Mathaudhu, "Dislocationtwin interactions in nanocrystalline fcc metals," Acta Materialia, vol. 59, pp. 812-821, 2011.

33. M.R. Barnett, "Twinning and the ductility of magnesium alloys: Part I: Tension twins," Materials Science and Engineering A, vol. 464, pp. 1-7, 2007.

34. L. Wu, S.R. Agnew, Y. Ren, D.W. Brown, B. Clausen, G.M. Stoica, H.R. Wenk and P.K. Liaw, "The effects of texture and extension twinning on the low-cycle fatigue behavior of a rolled magnesium alloy, AZ31B,” Materials Science and Engineering A, vol. 527, pp. 7057$7067,2010$.

35. E.W. Kelley and W.F. Hosford, "Plane-strain compression of magnesium and magnesium alloy crystals", Trans. The Minerals, Metals and Materials Society-AIME, vol. 242, pp. 5-13, 1968. 
36. S.E. Ion, F.J. Humphreys and S.H. White, "Dynamic recrystallization and the development of microstructure during the high temperature deformation of magnesium," Acta Metallurgica, vol. 30, pp. 1909-1919, 1982.

37. M. Niewczas, "Lattice correspondence during twinning in hexagonal close-packed crystals," Acta Materialia, vol. 58, pp. 5848-5857, 2010.

38. S. Kleiner and P.J. Uggowitzer, "Mechanical anisotropy of extruded Mg-6\%A1-1\%Zn alloy," Materials Science and Engineering A, vol. 379, pp. 258-263, 2004.

39. J. Koike, "Enhanced deformation mechanisms by anisotropic plasticity in polycrystalline Mg alloys at room temperature," Metallurgical and Materials Transactions A, vol. 36, pp. 16891696, 2005.

40. A. Jain and S.R. Agnew, "Effect of twinning on the mechanical behavior of a magnesium alloy sheet during strain path changes," in Magnesium Technology 2006, A.A. Luo, N.R. Neelameggham and R.S. Beals (Eds.), The Minerals, Metals and Materials Society, John Wiley \& Sons, Inc., Hoboken, New Jersey, 2006, pp. 219-224.

41. S.R. Agnew and O. Duygulu, "Plastic anisotropy and the role of non-basal slip in magnesium alloy AZ3 IB," International Journal of Plasticity, vol. 21, pp. 1161-1193, 2005.

42. B.L. Mordike and P. Lukáč, in Magnesium Technology 2006, A.A. Luo, N.R. Neelameggham and R.S. Beals (Eds.), the Minerals, Metals And Materials Society, John Wiley \& Sons, Inc., Hoboken, New Jersey, 2006, pp. 63-107.

43. F.H. Froes, K. Young-Won and S. Krishnamurthy, "Rapid solidification of lightweight metal alloys," in Micrometallurgy 88. Switzerland, 1989, pp. 19-32.

44. C.J. Bettles and M. Gibson, "Current wrought magnesium alloys: Strengths and weaknesses," JOM, vol. 57, pp. 46-49, 2005. 
45. B.L. Mordike, T. Ebert, "Magnesium properties-applications-potential," Materials Science and Engineering A, vol. 302, pp. 37-45, 2001.

46. E. Tenckhoff, Deformation mechanisms, texture, and anistropy in zirconium and Zircaloy. Philadelphia, PA: American Society for Testing and Materials, 1988.

47. M.H. Yoo, J.R. Morris, K.M. Ho and S.R. Agnew, "Nonbasal deformation modes of HCP metals and alloys: Role of dislocation source and mobility," Metallurgical and Materials Transactions A, vol. 33, pp. 813-822, 2002.

48. G.I. Taylor, "Plastic strain in metals," Institute of Metals - Journal, vol. 62, pp. 307-324, 1938.

49. P.G. Partridge, "The crystallography and deformation modes of hexagonal close-packed metals," Metallurgical Riviews, vol. 118, pp. 169-194, 1967.

50. S.H. Choi, E.J. Shin and B.S. Seong, "Simulation of deformation twins and deformation texture in an AZ31 Mg alloy under uniaxial compression," Acta Materialia, vol. 55, pp. 4181-4192, 2007.

51. T. Obara, H. Yoshinga and S. Morozumi, " $\{11 \overline{2} 2\}<\overline{1} \overline{1} 23>$ slip system in magnesium," Acta Metallurgica, vol. 21, pp. 845-853, 1973.

52. J.F. Stohr and J.P. Poirier, "Study by electron microscopy pyramidal slip $\{11 \overline{2} 2\}<\overline{1} \overline{1} 23>$ in magnesium," Philosophical Magzine A, vol. 25, pp. 1313-1329, 1972.

53. M.H. Yoo, "Slip, Twinning, and fracture in hexagonal close-packed metals," Metallurgical and Materials Transactions A, vol. 12, pp. 409-418, 1981.

54. A. Staroselsky and L. Anand, "A constitutive model for HCP materials deforming by slip and twinning: Application to magnesium alloy AZ31 B," International Journal of Plasticity, vol. 19, pp. 1843-1864, 2003. 
55. W.D. Callister Jr. and D.G. Rethwisch, Materials Science and Engineering: An Introduction. York, PA: John Wiley and Sons, Inc., 2008, pp. 103-104.

56. G.E. Dieter, Mechanical Metallurgy. New York, USA: McGraw-Hill Book Company, 1986.

57. W.F. Hosford, The Mechanics of Crystals and Textured Polycrystals. New York, USA: Oxford University Press, 1993, p. 163.

58. W.F. Hosford, The Mechanics of Crystals and Textured Polycrystals. New York, USA: Oxford University Press, 1993, pp. 172-185.

59. M.H. Yoo, S.R. Agnew, J.R. Morris and K.M. Ho, "Non-basal slip systems in HCP metals and alloys: Source mechanisms," Materials Science and Engineering A, vol. 319-321, pp. 8792, 2001.

60. W. Puschl, "Models for dislocation cross-slip in close-packed crystal structures: a critical review," Progress in Materials Science, vol. 47, pp. 415-461, 2002.

61. J.W. Christian and S. Mahajan, "Deformation twinning," Progress in Materials Science, vol. 39, pp. 1-157, 1995.

62. S. Mahajan and D.F. Williams, "Deformation Twinning in Metals and Alloys," International Metallurgical Reviews, vol. 18, pp. 43-61, 1973.

63. M.R. Barnett, Z. Keshavarz, A.G. Beer and D. Atwell, "Influence of grain size on the compressive deformation of wrought Mg-3Al-1Zn,” Acta Materialia, vol. 52, pp. 5093-5103, 2004.

64. L. Jiang, J.J. Jonas, A.A. Luo, A.K. Sachdev and S. Godet, “Twinning-induced Softening in polycrystalline AM30 Mg alloy at moderate temperatures," Scripta Materialia, vol. 54, pp. 771-775, 2006. 
65. S. Godet, L. Jiang, A.A. Luo and J.J. Jonas, "Use of Schmid factors to select extension twin variants in extruded magnesium alloy tubes," Scripta Materialia, vol. 55, pp. 1055-1058, 2006.

66. S.R. Agnew, D.W. Brown, S.C. Vogel and T.M. Holden, "In-situ measurement of internal strain evolution during deformation dominated by mechanical twinning," Materials Science Forum, vol. 404-407, pp. 747-754, 2002.

67. M.A. Gharghouri, G.C. Weatherly, J.K. Embury and J. Root, "Study of the mechanical properties of Mg-7.7 at.\% Al by in-situ neutron diffraction,” Philosophical Magazine A, vol. 79, pp. 1671-1695, 1999.

68. Z.S. Basinski, M.S. Szczerba, M. Niewczas, J.D. Embury and S.J. Basinski, "The transformation of slip dislocations during twinning of copper-aluminium alloy crystals," Revue de Metallurgie, vol. 94, pp. 1037-1043, 1997.

69. X.Y. Lou, M. Li, R.K. Boger, S.R. Agnew and R.H. Wagoner, "Hardening evolution of AZ31B Mg sheet,” International Journal of Plasticity, vol. 23, pp. 44-86, 2007.

70. W.H. Hartt and R.E. Reed-Hill, "Internal deformation and fracture at second-order $\{10 \overline{1} 1\}-$ $\{10 \overline{1} 2\}$ twins in magnesium," Trans. The Minerals, Metals and Materials Society-AIME, vol. 242, pp. 1127-1133, 1968.

71. H. Yoshinaga, T. Obara and S. Morozumi, "Twinning deformation in magnesium compressed along the c-axis," Materials Science and Engineering A, vol. 12, pp. 255-264, 1973.

72. L. Jiang, "Effect of twinning on texture and strain hardening in magnesium alloys subjected to different strain paths," In: Mining and Materials Engineering, Ph.D Thesis, McGill University, Montreal, 2007. 
73. M.R. Barnett, "Twinning and the ductility of magnesium alloys: Part II. Contraction twins," Materials Science and Engineering A, vol. 464, pp. 8-16, 2007.

74. R.E. Reed-Hill and W.D. Robertson, "Additional modes of deformation twinning in magnesium," Acta Metallurgica, vol. 5, pp. 717-727, 1957.

75. B.C. Wonsiewicz and W.A. Backofen, "Plasticity of magnesium crystals," Trans. The Minerals, Metals and Materials Society-AIME, vol. 239, pp. 1422-1431, 1967.

76. R.E. Reed-Hill, "A study of the $\{10 \overline{1} 1\}$ and $\{10 \overline{1} 3\}$ twinning modes in magnesium," Trans. The Minerals, Metals and Materials Society-AIME, vol. 218, pp. 554-558, 1960.

77. M.R. Barnett, "Twining and its role in magnesium alloys" in Advances in Wrought Magnesium Alloys: Fundamentals of processing, properties and applications, C. Bettles and M. Barnett (Eds.), Woodhead Publishing Limited, 2012, pp. 105-136.

78. R. Abbaschian, L. Abbaschian and R.E. Reed-Hill, Physical Metallurgy Principles. Stamford, USA: Cengage Learning, 2008.

79. L. Wu, "Mechanical behavior and the role of deformation twinning in wrought magnesium alloys investigated using neutron and synchrotron x-ray diffraction,” In: Materials Science and Engineering, Ph.D Thesis, The University of Tennessee, Knoxville, 2009.

80. H. Wang, P.D. Wu, J. Wang and C.N. Tomé, "A crystal plasticity model for hexagonal close packed (HCP) crystals including twinning and de-twinning mechanisms," International Journal of Plasticity, vol. 49, pp. 36-52, 2013.

81. L. Wu, S.R. Agnew, D.W. Brown, G.M. Stoica, B. Clausen, A. Jain, D.E. Fielden and P.K. Liaw, "Internal stress relaxation and load redistribution during the twinning-detwinningdominated cyclic deformation of a wrought magnesium alloy ZK60A," Acta Materialia, vol. 56, pp. 3699-3707, 2008a. 
82. L. Wu, S.R. Agnew, D.W. Brown, G.M. Stoica, B. Clausen, A. Jain, D.E. Fielden and P.K. Liaw, "Twinning-detwinning behavior during the strain controlled low-cycle fatigue testing of a wrought magnesium alloy ZK60A,” Acta Materialia, vol. 56, pp. 688-695, 2008b.

83. G. Proust, C.N. Tomé, A. Jain and S.R. Agnew, "Modeling the effect of twinning and detwinning during strain-path changes of magnesium alloy AZ31," International Journal of Plasticity, vol. 25, pp. 861-880, 2009.

84. Q. Yu, J.X. Zhang and Y.Y. Jiang, "Direct observation of twinning-detwinning-retwinning on magnesium single crystal subjected to strain-controlled cyclic tension-compression in [0001] direction,” Philosophical Magazine Letters, vol. 91, pp. 757-765, 2011.

85. J.X. Zhang, Q. Yu, Y.Y. Jiang and Q.Z. Li, “An experimental study of cyclic deformation of extruded AZ61A magnesium alloy,” International Journal of Plasticity, vol. 27, pp. 768-787, 2011.

86. C.S. Roberts, Magnesium and its Alloys. New York, USA: John Wiley \& Sons, Inc.,1960.

87. M.R. Barnett, “A Taylor model based description of the proof stress of magnesium AZ31 during hot working," Metallurgical and Materials Transactions A, vol. 34, pp. 1799-1806, 2003.

88. Y. Wu and W. Hu, "Comparison of the solid solution properties of $\mathrm{Mg}-\mathrm{RE}(\mathrm{Gd}, \mathrm{Dy}, \mathrm{Y})$ alloys with atomistic simulation," Research Letters in Physics, vol. 2008 pp. 476812: 1-4, 2008.

89. U.F. Kocks, C.N. Tomé and H.R. Wenk, Texture and Anisotropy: preferred orientations in polycrystals and their effect on materials properties. Cambridge, UK: Cambridge University Press, 1998. 
90. B.B. He, Two-Dimensional X-Ray Diffraction. Hoboken, New Jersey: John Wiley \& Sons, Inc., 2009.

91. G. Garcés, P. Pérez and P. Adeva, "Effect of the extrusion texture on the mechanical behaviour of Mg-SiCp composites," Scripta Materialia, vol. 52, pp. 615-619, 2005.

92. S.R. Agnew, M.H. Yoo and C.N. Tomé, "Application of texture simulation to understanding mechanical behavior of $\mathrm{Mg}$ and solid solution alloys containing Li or Y," Acta Materialia, vol. 49, pp. 4277-4289, 2001.

93. P. Okrutny, "Modelling of recovery and recrystallization in magnesium alloys," In: Materials Science and Engineering, MS.c Thesis, McMaster University, Hamilton, 2010.

94. Y. Chino, K. Kimura and M. Mabuchi, "Deformation characteristics at room temperature under biaxial tensile stress in textured AZ31 Mg alloy sheets," Acta Materialia, vol. 57, pp. 1476-1485, 2009.

95. E.W. Kelly and W.F. Hosford Jr., "The deformation characteristics of textured magnesium," Transactions of Metallurgical Society of AIME, vol. 242, pp. 654-661, 1968.

96. M.M. Avedesian and H. Baker, Magnesium and Magnesium Alloys. Materials Park, OH: ASM International, 1999.

97. Y.N. Wang and J.C. Huang, "The role of twinning and untwinning in yielding behavior in hot-extruded Mg-Al-Zn alloy,” Acta Materialia, vol. 55, pp. 897-905, 2007.

98. D.W. Brown, J.D. Almer, B. Clausen, P.L. Mosbrucker, T.A. Sisneros and S.C. Vogel, “Twinning and de-twinning in beryllium during strain path changes, ” Materials Science and Engineering A, vol. 559, pp. 29-39, 2013. 
99. S.G. Hong, S.H. Park and C.S. Lee, "Enhancing the fatigue property of rolled AZ31 magnesium alloy by controlling $\{10 \overline{1} 2\}$ twinning-detwinning characteristics," Journal of Materials Research, vol. 25, pp. 784-792, 2010.

100. J. He, T. Liu, Y. Zhang and J. Tan, "Twin characteristics and flow stress evolution in extruded magnesium alloy AZ31 subjected to multiple loads," Journal of Alloys Compounds, vol. 578, pp. 536-542, 2013.

101. S.M. Yin, H.J. Yang, S.X. Li, S.D. Wu and F. Yang, "Cyclic deformation behavior of asextruded Mg-3\%Al-1\%Zn,” Scripta Materialia, vol. 58, pp. 751-754, 2008.

102. P. Molnár, A. Ostapovets and A. Jäger, "Reversible motion of twin boundaries in AZ31 alloy and new design of magnesium alloys as smart materials," Materials and Design, vol. 56, pp. 509-516, 2014.

103. Y. Xin, J. Jiang, A. Chapuis, M. Wang and Q. Liu, "Plastic deformation behavior of AZ31 magnesium alloy under multiple passes cross compression," Materials Science and Engineering A, vol. 532, pp. 50-57, 2012.

104. S.M. Fatemi-Varzaneh, A. Zarei-Hanzaki, and H. Beladi, "Dynamic recrystallization in AZ31 magnesium alloy," Materials Science and Engineering A, vol. 456, pp. 52-57, 2007.

105. T. Al-Samman, and G. Gottstein, "Dynamic recrystallization during high temperature deformation of magnesium," Materials Science and Engineering A, vol. 490, pp 411-420, 2008.

106. T. Al-Samman, K.D. Molodov, D.A. Molodov, G. Gottstein and S. Suwas, "Softening and dynamic recrystallization in magnesium single crystals during $c$-axis compression," Acta Materialia, vol. 60, pp. 537-545, 2012. 
107. Q. Ma, B. Li, W.R. Whittington, A.L. Oppedal, P.T. Wang and M.F. Horstemeyer, "Texture evolution during dynamic recrystallization in a magnesium alloy at $450^{\circ} \mathrm{C}$," Acta Materialia , vol. 67, pp. 102-115, 2014.

108. X. Li, P. Yang, L.-N. Wang, L. Meng and F. Cui, "Orientational analysis of static recrystallization at compression twins in a magnesium alloy AZ31," Materials Science and Engineering A, vol. 517, pp. 160-169, 2009.

109. J. Zou, J. Jain, and C.W. Sinclair, "Tracing nucleation and grain growth during static recrystallization of pure Mg by EBSD," in Magnesium Technoligy 2009, E.A. Nyberg, S.R. Agnew, N.R. Neelameggham, M.O. Pekguleryuz (Eds.), The Minerals, Metals And Materials Society, John Wiley \& Sons, Inc., Hoboken, New Jersey, 2006, pp. 13-17.

110. J.X. Zou, C.W. Sinclair and F. Wagner, "The relationship between microstructure, texture and static recrystallization in uniaxially compressed magnesium," in 8th International Conference on Magnesium and its Alloys. 2009. Weimar, Germany.

111. F.J. Humphreys and M. Hatherly, Recrystallization and Related Annealing Phenomena. Amsterdam: Elsevier, 2004.

112. M. Knezevic, A. Levinson, R. Harris, R.K. Mishra, R.D. Doherty and S.R. Kalidindi, "Deformation twinning in AZ31: Influence on strain hardening and texture evolution," Acta Materialia, vol. 58, pp. 6230-6242, 2010.

113. A. Levinson, R.K. Mishra, R.D. Doherty and S.R. Kalidindi, "Influence of deformation twinning on static annealing of AZ31Mg alloy," Acta Materialia, vol. 61, pp. 5966-5978, 2013. 
114. X. Yang, Y. Okabe, H. Miura and T. Sakai, "Effect of prior strain on continuous recrystallization in AZ31 magnesium alloy after hot deformation," Materials Science and Engineering A, vol. 535, pp. 209-215, 2012.

115. A. Rohatgi, K.S. Vecchio, and G.T. Gray III, "The influence of stacking fault energy on the mechanical behavior of $\mathrm{Cu}$ and $\mathrm{Cu}-\mathrm{Al}$ alloys: deformation twinning, work hardening, and dynamic recovery," Metallurgical and Materials Transactions A, vol. 32, pp. 135-141, 2001.

116. L. Jiang, J.J. Jonas, A.A. Luo, A.K. Sachdev and S. Godet, "Influence of $\{10 \overline{1} 2\}$ extension twinning on the flow behavior of AZ31 Mg alloy," Materials Science and Engineering A, vol. 445-446, pp. 302-309, 2007.

117. F. Bachmann, R. Hielscher and H. Schaeben, "Texture analysis with MTEX free and open source software toolbox," Solid State Phenomena, vol. 160, pp. 63-68, 2010.

118. S.G. Hong, S.H. Park and C.S. Lee, "Role of $\{10 \overline{1} 2\}$ twinning characteristics in the deformation behavior of a polycrystalline magnesium alloy," Acta Materialia, vol. 58, pp. $5873-5885,2010$.

119. N. Li, J. Wang, A. Misra, X. Zhang, J.Y. Huang and J.P. Hirth, "Twinning dislocation multiplication at a coherent twin boundary," Acta Materialia, vol. 59, pp. 5989-5996, 2011.

120. H. El-Kadiri, J. Kapil, A.L. Oppedal, L.G. Hector Jr., S.R. Agnew, M. Cherkaoui and S.C. Vogel, "The effect of twin-twin interactions on the nucleation and propagation of image twinning in magnesium," Acta Materialia, vol. 61, pp. 3549-3563, 2013.

121. L. Capolungo, I.J. Beyerlein, G.C. Kaschner and C.N. Tomé, "On the interaction between slip dislocations and twins in HCP Zr," Materials Science and Engineering A, vol. 513-514, pp. 42-51, 2009. 
122. H. Wang, P.D. Wu, C.N. Tomé and J. Wang, "A constitutive model of twinning and detwinning for hexagonal close packed polycrystals," Materials Science and Engineering A, vol. 555, pp. 93-98, 2012.

123. R.Y. Zhang, M.R. Daymond, and R.A. Holt, "A finite element model of deformation twinning in zirconium," Materials Science and Engineering A, vol. 473, pp. 139-146, 2008.

124. H.A. Patel, D.L. Chen, S.D. Bhole and K. Sadayappan, "Cyclic deformation and twinning in a semi-solid processed AZ91D magnesium alloy," Materials Science and Engineering A, vol. 528, pp. 208-219, 2010.

125. D. Sarker, J. Friedman and D.L. Chen, "Influence of pre-strain on de-twinning activity in an extruded AM30 magnesium alloy," Materials Science and Engineering A, vol. 605, pp. 7379, 2014.

126. H.A. Patel, N. Rashidi, D.L. Chen, S.D. Bhole and A.A. Luo, "Cyclic deformation behavior of a super-vacuum die cast magnesium alloy," Materials Science and Engineering A, vol. 546, pp. 72-78, 2012.

127. A.A. Salem, S.R. Kalidindi, R.D. Doherty and S.L. Semiatin, "Strain hardening due to deformation twinning in $\alpha$-titanium: Mechanisms," Metallurgical and Materials Transactions A, vol. 37, pp. 259-268, 2006.

128. B.S. Wang, R.L. Xin, G.J. Huang and Q. Liu, "Effect of crystal orientation on the mechanical properties and strain hardening behavior of magnesium alloy AZ31 during uniaxial compression," Materials Science and Engineering A, vol. 534, pp. 588-593, 2012.

129. D. Sarker and D.L. Chen, "Texture transformation in an extruded magnesium alloy under pressure," Materials Science and Engineering A, vol. 582, pp. 63-67, 2013. 
130. M.B. Chen, J. Li, Y.M. Zhao, H. Yuan and W.C. Liu, "Comparison of texture evolution between different thickness layers in cold rolled Al-Mg alloy," Materials Characterization, vol. 62, pp. 1188-1195, 2011.

131. W.C. Liu and J.G. Morris, "Comparison of the texture evolution in cold rolled DC and SC AA 5182 aluminum alloys," Materials Science and Engineering A, vol. 339, pp. 183-193, 2003.

132. W.C. Liu and J.G. Morris, "Quantitative analysis of texture evolution in cold-rolled, continuous-cast AA 5xxx-series aluminum alloys," Metallurgical Transections A, vol. 35, pp. 265-277, 2004.

133. C.L. Fan, D.L. Chen and A.A. Luo, "Dependence of the distribution of deformation twins on strain amplitudes in an extruded magnesium alloy after cyclic deformation," Materials Science and Engineering A, vol. 519, pp. 38-45, 2009.

134. J. Hirsch and T. Al-Samman, "Superior light metals by texture engineering: Optimized aluminum and magnesium alloys for automotive applications," Acta Materialia, vol. 61, pp. 818-843, 2013.

135. D. Sarker and D.L. Chen, "Detwinning and strain hardening of an extruded magnesium alloy during compression," Scripta Materialia, vol. 67, pp. 165-168, 2012.

136. Q. Ma, H. El-Kadiri, A.L. Oppedal, J.C. Baird, B. Li, M.F. Horstemeyer and S.C. Vogel, "Twinning effects in a rod-textured AM30 Magnesium alloy," International Journal of Plasticity, vol. 29, pp. 60-76, 2012.

137. J. Wang, L. Liu, C.N. Tomé, S.X. Mao and S.K. Gong, "Twinning and de-twinning via glide and climb of twinning dislocations along serrated coherent twin boundaries in hexagonalclose-packed metals," Materials Research Letter, vol. 1, pp. 81-88, 2013. 
138. D. Ji, C. Liu, L. Tang, Y. Wan and C. Huang, "Microstructures and mechanical properties of a hot extruded Mg-4.45Zn-0.46Y-0.76Zr alloy plate," Materials and Design, vol. 53, pp. 602-610, 2014.

139. S. Biswas, S.S. Dhinwal and S. Suwas, "Room-temperature equal channel angular extrusion of pure magnesium," Acta Materialia, vol. 58, pp. 3247-3261, 2010.

140. I. Ulacia, N.V. Dudamell, F. Gálvez, S. Yi, M.T. Pérez-Prado and I. Hurtado, "Mechanical behavior and microstructural evolution of a Mg AZ31 sheet at dynamic strain rates," Acta Materialia, vol. 58, pp. 2988-2998, 2010.

141. L. Lu, T. Liu, M.J. Tan, J. Chen and Z. Wang, "Effect of annealing on microstructure evolution and mechanical property of cold forged magnesium pipes," Materials and Design, vol. 39, pp. 131-139, 2012.

142. J.F. Bingert, T.A. Mason, G.C. Kaschner, P.J. Maudlin and G.T. Gray III, "Deformation twinning in polycrystalline $\mathrm{Zr}$ : insights from electron backscattered diffraction characterization," Metallurgical and Materials Transactions A, vol. 33, pp. 955-963, 2002.

143. L.W.F. Mackenzie and M.O. Pekguleryuz, "The recrystallization and texture of magnesiumzinc-cerium alloys," Scripta Materialia, vol. 59, pp. 665-668, 2008.

144. N. Stanford and M.R. Barnett, "The origin of "rare earth" texture development in extruded Mg-based alloys and its effect on tensile ductility," Materials Science and Engineering A, vol. 496, pp. 399-408, 2008.

145. Y. Chino, K. Sassa and M. Mabuchi, "Texture and stretch formability of a rolled Mg-Zn alloy containing dilute content of Y," Materials Science and Engineering A, vol. 513-514, pp. 394-400, 2009. 
146. T. Mukai, M. Yamanoi, H. Watanabe and K. Higashi, "Ductility enhancement in AZ31 magnesium alloy by controlling its grain structure," Scripta Materialia, vol. 45, pp. 89-94, 2001.

147. W.J. Kim, C.W. An, Y.S. Kim and S.I. Hong, "Mechanical properties and microstructures of an AZ61 Mg alloy produced by equal channel angular pressing, ” Sctipta Materialia, vol. 47, pp. 39-44, 2002.

148. Y. Yoshida, L. Cisar, S. Kamado and Y. Kojima, "Effect of microstructural factors on tensile properties of an ECAE-processed AZ31 magnesium alloy," Materials Transactions, vol. 44, pp. 468-475, 2003.

149. S.R. Agnew, P. Mehrotra, T.M. Lillo, G.M. Stoica and P.K. Liaw, "Crystallographic texture evolution of three wrought magnesium alloys during equal channel angular extrusion," Materials Science and Engineering A, vol. 408, pp. 72-78, 2005.

150. Y. Chino, K. Sassa and M. Mabuchi, "Enhancement of tensile ductility of magnesium alloy produced by torsion extrusion," Scripta Materialia, vol. 59, pp. 399-402, 2008.

151. X. Huang, K. Suzuki, A. Watazu, I. Shigematsu and N. Saito, "Mechanical properties of MgAl-Zn alloy with a tilted basal texture obtained by differential speed rolling," Materials Science and Engineering A, vol. 488, pp. 214-220, 2008.

152. B.L. Wu, Y.H. Zhao, X.H. Du, Y.D. Zhang, F. Wagner and C. Esling, "Ductility enhancement of extruded magnesium via yttrium addition," Materials Science and Engineering A, vol. 527, pp. 4334-4340, 2010.

153. Q. Ma, H. El-Kadiri, A.L. Oppedal, J.C. Baird, M.F. Horstemeyer and M. Cherkaoui, "Twinning and double twinning upon compression of prismatic textures in an AM30 magnesium alloy," Scripta Materialia, vol. 64, pp. 813-816, 2011. 
154. E. Bertrand, P. Castany, I. Péron and T. Gloriant, "Twinning system selection in a metastable $\beta$-titanium alloy by Schmid factor analysis," Scripta Materialia, vol. 64, pp. 1110-1113, 2011.

155. S.-G. Hong, S.H. Park and C.S. Lee, "Strain path dependence of $\{10 \overline{1} 2\}$ twinning activity in a polycrystalline magnesium alloy," Scripta Materialia, vol. 64, pp. 145-148, 2011.

156. S.H. Park, S.-G. Hong and C.S. Lee, "Activation mode dependent $\{1012\}$ twinning characteristics in a polycrystalline magnesium alloy," Scripta Materialia, vol. 62, pp. 202$205,2010$.

157. H. Wang, P.D. Wu and M.A. Gharghouri, "Effects of basal texture on mechanical behaviour of magnesium alloy AZ31B sheet," Materials Science and Engineering A, vol. 527, pp. 3588-3594, 2010.

158. X.G. Deng, S.X. Hui, W.J. Ye and X.Y. Song, "Analysis of twinning behavior of pure Ti compressed at different strain rates by Schmid factor," Materials Science and Engineering A, vol. 575, pp. 15-20, 2013.

159. X.-L. Nan, H.-Y. Wang, L. Zhang, J.-B. Li and Q.-C. Jiang, "Calculation of Schmid factors in magnesium: analysis of deformation behaviors," Scripta Materialia, vol. 67, pp. 443-446, 2012.

160. X.-L. Nan, H.-Y. Wang, Z.-Q. Wu, E.-S. Xue, L. Zhang and Q.-C. Jiang, "Effect of c/a axial ratio on Schmid factors in hexagonal close-packed metals," Scripta Materialia, vol. 68, pp. 530-533, 2013.

161. C.J. Boehlert, Z. Chen, I. Gutiérrez-Urrutia, J. Llorca and M.T. Pérez-Prado, "In situ analysis of the tensile and tensile-creep deformation mechanisms in rolled AZ31," Acta Materialia, vol. 60, pp. 1889-1904, 2012. 
162. G. Thomas and M.J. Goringe, Transmission Electron Microscopy of Materials. New York, USA: John Wiley and Sons, 1979.

163. G.-S. Song, S.-H. Zhang, L. Zheng and L. Ruan, "Twinning, grain orientation and texture variation of AZ31 Mg alloy during compression by EBSD tracing," Journal of Alloys and Compounds, vol. 509, pp. 6481-6488, 2011.

164. J. Koike and R. Ohyama, "Geometrical criterion for the activation of prismatic slip in AZ61 Mg alloy sheets deformed at room temperature," Acta Materialia, vol. 53, pp.1963-1972, 2005.

165. S.R. Agnew, J.A. Horton, T.M. Lillo and D.W. Brown, "Enhanced ductility in strongly textured magnesium produced by equal channel angular processing," Scripta Materialia, vol. 50, pp. 377-381, 2004.

166. M.R. Barnett, Z. Keshavarz and X. Ma, "A semi analytical sachs model for the flow stress of a magnesium alloy," Metallurgical and Materials Transactions A, vol. 37A, pp. 2283-2293, 2006.

167. F.A. Mirza and D.L. Chen, "Fatigue of magnesium alloys" in Aerospace Materials Handbook, eds S. Zhang, D.L. Zhao, New York, USA: CRC Press, Taylor \& Francis, 2013, pp. 647-698.

168. N. Afrin, D.L. Chen, X. Cao and M. Jahazi, "Strain hardening behavior of a friction stir welded magnesium alloy," Scripta Materialia, vol. 57, pp. 1004-1007, 2007.

169. D. Sarker and D.L. Chen, "Dependence of compressive deformation on pre-strain and loading direction in an extruded magnesium alloy: Texture, twinning and de-twinning," Materials Science and Engineering A, vol. 596, pp. 134-144, 2014. 
170. R.B. Figueiredo, Z. Száraz, Z. Trojanová, P. Lukáč and T.G. Langdon, "Significance of twinning in the anisotropic behavior of a magnesium alloy processed by equal-channel angular pressing," Scripta Materialia, vol. 63, pp. 504-507, 2010.

171. D. Sarker, J. Friedman and D.L. Chen, "Twin growth and texture characteristics in an extruded AM30 magnesium alloy during compression," Journal of Materials Science and Technology, In Press.

172. D. Sarker and D.L. Chen, "Texture development in an extruded magnesium alloy during compression along the transverse direction," in Magnesium Technology 2013, N. Hort, S.N. Mathaudhu, N.R. Neelameggham and M. Alderman (Eds.), The Minerals, Metals And Materials Society, John Wiley \& Sons, 2013, pp. 313-316.

173. L. Jiang and J.J. Jonas, "Effect of twinning on the flow behavior during strain path reversals in two Mg (+Al, Zn, Mn) alloys," Scripta Materialia, vol. 58, pp. 803-806, 2008.

174. N.V. Dudamell, I. Ulacia, F. Gálvez, S. Yi, J. Bohlen, D. Letzig, I. Hurtado and M.T. PérezPrado, "Twinning and grain subdivision during dynamic deformation of a Mg AZ31 sheet alloy at room temperature," Acta Materialia, vol. 59, pp. 6949-6962, 2011.

175. J.A. del Valle, F. Carreño, and O.A. Ruano, "Influence of texture and grain size on work hardening and ductility in magnesium-based alloys processed by ECAP and rolling," Acta Materialia, vol. 54, pp. 4247-4259, 2006.

176. D. Sarker and D.L. Chen, "The orientation dependence of strain hardening and texture development in an extruded magnesium alloy," Materials Science Forum, vol. 783-786, pp. 363-368, 2014. 
177. J. Dallmeier, O. Huber, H. Saage, K. Eigenfeld, and A. Hilbig, "Quasi-static and fatigue behavior of extruded ME21 and twin roll cast AZ31 magnesium sheet metals," Materials Science and Engineering A, vol. 590, pp. 44-53, 2014.

178. C.H. Cáceres, T. Sumitomo and M. Veidt, "Pseudoelastic behaviour of cast magnesium AZ91 alloy under cyclic loading-unloading,” Acta Materialia, vol. 51, pp. 6211-6218, 2003.

179. U.F. Kocks and H. Mecking, "Physics and phenomenology of strain hardening: the FCC case," Progress in Materials Science, vol. 48, pp. 171-273, 2003.

180. R. Kapoor, A. Sarkar, J. Singh, I. Samajdar and D. Raabe, "Effect of strain rate on twinning in a Zr alloy," Scripta Materialia, vol. 74, pp. 72-75, 2014.

181. Q. Yu, J. Wang, Y.Y. Jiang, R.J. McCabe and C.N. Tomé, “Co-zone $\{10 \overline{1} 2\}$ twin interaction in magnesium single crystal," Materials Research Letters, vol. 2, pp. 82-88, 2014.

182. J.H. Cho, S.S. Jeong, H.W. Kim and S.B. Kang, "Texture and microstructure evolution during the symmetric and asymmetric rolling of AZ31B magnesium alloys," Materials Science and Engineering A, vol. 566, pp. 40-46, 2013.

183. A.L. Oppedal, H.El Kadiri, C.N. Tomé, J.C. Baird, S.C. Vogel and M.F. Horstemeyer, "limitation of current hardening models in predicting anisotropy by twinning in hep metals: Application to a Rod-textured AM30 magnesium alloy," in Magnesium Technology 2011, W.H. Sillekens, S.R. Agnew, N.R. Neelameggham, S.N. Mathaudhu (Eds.), John Wiley \& Sons, Inc., Hoboken, New Jersey, 2011, pp. 313-320.

184. Q. Ma, B. Li, W.R. Whittington, A.L. Oppedal, P.T. Wang and M.F. Horstemeyer, "Texture evolution during dynamic recrystallization in a magnesium alloy at $450^{\circ} \mathrm{C}$," Acta Materialia, vol. 67, pp. 102-115, 2014. 
185. Z. Zhang, "The formation of double peaks in the basal texture during ambient extrusion of an AZ31 magnesium alloy,” Materials Letters, vol. 116, pp. 131-134, 2014.

186. M. Kohzu, K. Kii, Y. Nagata, H. Nishio, K. Higashi and H. Inoue, "Texture randomization of AZ31 magnesium alloy sheets for improving the cold formability by a combination of rolling and high-temperature annealing," Materials Transactions, vol. 51, pp. 749-755, 2010.

187. F.J. Humphreys and M. Hatherly, Recrystallization and Related Annealing Phenomena. Kidlington, Oxford, UK: Elsevier, 2004.

188. R.D. Doherty, D.A. Hughes, F.J. Humphreys, J.J. Jonas, D.J. Jensen, M.E. Kassner, W.E. King, T.R. McNelley, H.J. McQueen and A.D. Rollett, "Current issues in recrystallization: a review," Materials Science and Engineering A, vol. 238, pp. 219-274, 1997.

189. C.W. Su, L. Lu and M.O. Lai, "Recrystallization and grain growth of deformed magnesium alloy," Philosophical Magazine, vol. 88, pp. 181-200, 2008.

190. M.T. Pérez-Prado, J.A. del Valle, J.M. Contreras and O.A. Ruano, "Microstructural evolution during large strain hot rolling of an AM60 Mg alloy," Scripta Materialia, vol. 50, pp. 661$665,2004$.

191. D.L. Yin, K.F. Zhang, G.F. Wang and W.B. Han, "Warm deformation behavior of hot-rolled AZ31 Mg alloy," Materials Science and Engineering A, vol. 392, pp. 320-325, 2005.

192. Y.V.R.K. Prasad and K.P. Rao, "Effect of crystallographic texture on the kinetics of hot deformation of rolled Mg-3Al-1Zn alloy plate," Materials Science and Engineering A, vol. 432, pp. 170-177, 2006.

193. J.A. del Valle and O.A. Ruano, "Influence of texture on dynamic recrystallization and deformation mechanisms in rolled or ECAPed AZ31 magnesium alloy," Materials Science and Engineering A, vol. 487, pp. 473-480, 2008. 
194. P. Klimanek and A. Pötzsch, "Microstructure evolution under compressive plastic deformation of magnesium at different temperatures and strain rates," Materials Science and Engineering A, vol. 324, pp. 145-150, 2002.

195. M.D. Nave and M.R. Barnett, "Microstructures and textures of pure magnesium deformed in plane-strain compression," Scripta Materialia, vol. 51, pp. 881-885, 2004.

196. A.B. Ma, J.H. Jiang, N. Saito, I. Shigematsu, Y.C. Yuan, D.H. Yang and Y. Nishida, "Improving both strength and ductility of a Mg alloy through a large number of ECAP passes," Materials Science and Engineering A, vol. 513-514, pp. 122-127, 2009.

197. W.J. Kim, S.I. Hong, Y.S. Kim, S.H. Min, H.T. Jeong and J.D. Lee, "Texture development and its effect on mechanical properties of an AZ61 Mg alloy fabricated by equal channel angular pressing,” Acta Materialia, vol. 51, pp. 293-307, 2003.

198. J. Xing, H. Soda, X.Y. Yang, H. Miura and T. Sakai, "Ultra-fine grain development in an AZ31 magnesium alloy during multi-directional forging under decreasing temperature conditions," Materials Transections, vol. 46, pp. 1646-1650, 2005.

199. S.M. Fatemi-Varzaneh and A. Zarei-Hanzaki, "Processing of AZ31 magnesium alloy by a new noble severe plastic deformation method," Materials Science and Engineering A, vol. 528, pp. 1334-1339, 2011.

200. L.W. Lu, T.M. Liu, Y. Chen, L.G. Wang and Z.C. Wang, "Double change channel angular pressing of magnesium alloys AZ31," Materials and Design, vol. 35, pp. 138-143, 2012.

201. L.W. Lu, T.M. Liu, S. Jiang, F.S. Pan, Q. Liu and Z.C. Wang, "Microstructure and mechanical property of dual-directional-extruded Mg alloy AZ31," Materials Science and Engineering A, vol. 527, pp. 4050-4055, 2010. 
202. K.P. Rao, Y.V.R.K. Prasad and K. Suresh, "Materials modeling and simulation of isothermal forging of rolled AZ31B magnesium alloy: Anisotropy of flow," Materials and Design, vol. 32, pp. 2545-2553, 2011.

203. S. Das, N.S. Lim, J.B. Seol, H.W. Kim and C.G. Park, "Effect of the rolling speed on microstructural and mechanical properties of aluminum-magnesium alloys prepared by twin roll casting," Materials and Design, vol. 31, pp. 1633-1638, 2010.

204. H.Y. Chao, H.F. Sun, W.Z. Chen and E.D. Wang, "Static recrystallization kinetics of a heavily cold drawn AZ31 magnesium alloy under annealing treatment," Materials Characterization, vol. 62, pp. 312-320, 2011.

205. X.H. Chen, F.S. Pan, J.J. Mao, J.F. Wang, D.F. Zhang, A.T. Tang and J. Peng, "Effect of heat treatment on strain hardening of ZK60 Mg alloy," Materials and Design, vol. 32, pp. 1526-1530, 2011.

206. A. Jager, P. Lukac, V. Gartnerova, J. Haloda and M. Dopita, "Influence of annealing on the microstructure of commercial Mg alloy AZ31 after mechanical forming," Materials Science and Engineering A, vol. 432, pp. 20-25, 2006.

207. X. Yang, H. Miura and T. Sakai, "Structural development at severely high strain in AZ31 magnesium alloy processed by cold forging and subsequent annealing," Materials and Design, vol. 44, pp. 573-579, 2013.

208. X. Yang, Y. Okabe, H. Miura and T. Sakai, "Annealing of a magnesium alloy AZ31 after interrupted cold deformation," Materials and Design, vol. 36, pp. 626-632, 2012.

209. M. Oyarzábal, A. Martínez-de-Guerenu and I. Gutiérrez, "Effect of stored energy and recovery on the overall recrystallization kinetics of a cold rolled low carbon steel," Materials Science and Engineering A, vol. 485, pp. 200-209, 2008. 
210. I. Samajdar, B. Verlinden, P. Van Houtte and D. Vanderschueren, "Recrystallisation kinetics in IF-steel: A study on the sluggish recrystallisation behaviour," Scripta Materialia, vol. 37, pp. 869-874, 1997.

211. X. Wu, X. Yang, J. Ma, Q. Huo, J. Wang and H. Sun, "Enhanced stretch formability and mechanical properties of a magnesium alloy processed by cold forging and subsequent annealing," Materials and Design, vol. 43, pp. 206-212, 2013.

212. D. Sarker, J. Friedman and D.L. Chen, "Influence of pre-deformation and subsequent annealing on strain hardening and anisotropy of AM30 magnesium alloy," Journal of Alloys and Compounds, vol. 611, pp. 341-350, 2014. 